\title{
DEVELOPMENT OF SPRAY AND COMBUSTION MODELS FOR THE SIMULATION OF GAS TURBINE COMBUSTION SYSTEMS
}

Enric Illana Mahiques 


\section{Composition of the graduation committee:}

Chairman and secretary:

Prof. dr. G.P.M.R. Dewulf University of Twente

Promotor:

Prof. dr. ir. T.H. van der Meer University of Twente Co-promotor:

Dr. ir. J.B.W Kok

University of Twente

Special expert:

Dr. C. Beck

Siemens AG

Members:

Prof. dr. ir. B.J. Geurts

University of Twente

Prof. dr. S.R.A. Kersten

University of Twente

Prof. dr. D.J.E.M. Roekaerts

Delft University of Technology

Dr. ir. J.A. van Oijen

Eindhoven University of Technology

This research was financially supported by the European Commission through the Marie Curie Actions - Networks for Initial Training program, under call FPr-PEOPLE-2011ITN, Project COPA-GT, with project number 290042.

(C) Enric Illana Mahiques, 2016

No part of this publication may be reproduced by print, photocopy or any other means without the permission of the copyright owner.

ISBN: 978-90-365-4292-0

DOI: $10.3990 / 1.9789036542920$

Keywords: Liquid fuel, Modelling, Atomization, Evaporation, Combustion, Tabulated Chemistry, Water-in-Oil Emulsion, Jet Flame, Acoustic Response 


\section{DEVELOPMENT OF SPRAY AND COMBUSTION MODELS FOR THE SIMULATION OF GAS TURBINE COMBUSTION SYSTEMS}

\section{DISSERTATION}

to obtain

the degree of doctor at the University of Twente, on the authority of the rector magnifictus, Prof. dr. T.T.M. Palstra, to be publicly defended on Thursday the $9^{\text {th }}$ of February 2017 at 12:45 h.

by

Enric Illana Mahiques

born on the $20^{\text {th }}$ of December 1989

in Valencia, Spain 
This dissertation has been approved by:

The promotor:

Prof. dr. ir. T.H. van der Meer University of Twente

The co-promotor:

Dr. ir. J.B.W Kok

University of Twente 


\section{Abstract}

A tool for the simulation of gas turbine combustors during oil operation is build within OpenFOAM by modelling the main processes involved.

A primary atomization model for sprays in cross flow has been further developed for Large-Eddy simulations. Cylindrical droplets with the size of the injector hole are introduced in the domain and atomized as they penetrate into the cross flow. Classic two-way coupling methods require a high cell-to-droplet volume ratio, otherwise stability issues or non-physical results can potentially arise. To avoid this limitation, a novel coupling method is developed to distribute the source terms to the cells that are within a specific distance from the parcel. Those cells are also used for the interpolation of gas phase properties at the droplet position. After a calibration and a mesh sensitivity study, the combined primary atomization and coupling models are validated for kerosene jet A-1 sprays at several operation conditions.

An evaporation model for multicomponent fuels and emulsions has also been implemented within the Euler-Lagrangian formulation. The model is suitable for pressures typical of heavy duty gas turbines and assumes an increased evaporation rate of the most volatile component when the droplet temperature reaches its boiling temperature. Evaporation of emulsion droplets is modelled following a shell approach, assuming that the water in the droplet core does not evaporate. A diffusivity model is introduced to account for the transport of the dispersed phase from the core to the outer shell. Experimental data for $n$-heptane and n-decane droplets under several ambient temperatures and pressures are used for validation.

The combustion model is based on the Flamelet Generated Manifold approach. The mixture fraction is calculated from the species mass fractions to couple it with the evaporation model. Flamelet tables are created for different enthalpies and mixture fractions so that they can be used in non-premixed simulations. The thermo-chemistry model considers up to two fuel components by using 4-D tables. One-dimensional results, in terms of laminar flame speed and main species profiles, from the combustion model are compared with the detailed chemistry solutions for hexadecane-water-air mixtures at two pressure levels.

Following the separate evaluation of each model, coupled simulations are performed to analyse their interaction and to verify that they can be used in the simulation of 
partially pre-vaporized spray flames. Evaporating sprays with different momentum ratios determine the impact of spray effects on the amount of evaporated mass, while 1-D spray flames show the influence of the spray statistics on the flame position. Effect of water addition is also investigated for both configurations.

Numerical simulations of a jet tube combustor at pressurized conditions are performed for different fuel mixtures, including liquid fuels. Experimental data measured at the DLR Institute of Combustion Technology is used as a reference. For gaseous fuel mixtures, simulation results are compared with $\mathrm{OH}$-chemiluminescence images and PIV measurement data. Both flame length and lift are qualitatively well predicted, and the change in flame intensity from 0 to $40 \%$ propane mass content is properly captured. Velocity profiles within the chamber are in good agreement with the experiments. For liquid fuel cases, simulations are qualitatively compared with $\mathrm{OH}$-chemiluminescence and Mie scattering images. As in the experiment, a low amount of non-vaporized mass flow at the entrance of the combustion chamber is observed. Compared to gas operation, the flame is more stable and intense. The addition of water leads to a slower spray evaporation, a greater spray-wall interaction, a reduced gas phase temperature and a less intense flame. A higher air inlet temperature increases the evaporation rate and the flame is more stable.

Subsequently, a forced response approach that uses mono-frequency excitations at the inlet boundary is employed to determine the acoustic response of each model individually. The atomization and evaporation processes are enhanced by acoustic waves due to their dependency on the relative velocity. The mean laminar speed and thickness of a premixed flame are not significantly affected by acoustics, having the former a larger response. Forcing is also applied to coupled simulations, so that acoustic response of evaporating sprays as well as 1-D spray flames is evaluated. Following this, the response of the jet tube combustor operating with both liquid and gaseous fuels is analysed. 


\section{Samenvatting}

Binnen OpenFOAM is een tool gebouwd om verbrandingskamers in gasturbines tijdens het gebruik van olie te simuleren door het modelleren van de voornaamste relevante processen.

Daarnaast is er een model voor de primaire verstuiving van een brandstofstraal, loodrecht geinjecteerd in een luchtstroom, ontwikkeld voor "large eddy"-simulaties. Er worden cilindrische druppeltjes ter grootte van het injectiegat ingebracht, die verstuiven zodra zij de luchtstroom binnenkomen. Voor een conventionele tweerichtingskoppelmethode is een hoge volumeverhouding tussen cellen en druppeltjes vereist, omdat zich anders stabiliteitsproblemen of andere niet-fysische verschijnselen kunnen voordoen. Om dat te voorkomen, is er een nieuwe koppelmethode ontwikkeld om de brontermen te distribueren over cellen die zich op een bepaalde afstand van het druppel pakketje bevinden. Diezelfde cellen worden gebruikt voor de gasfase-interpolatie op de positie van het druppeltje. Na calibratie en een rekenrooster gevoeligheidsonderzoek worden de gecombineerde modellen voor primaire verstuiving en koppeling gevalideerd voor stralen Jet A-1-kerosine onder verschillende operationele omstandigheden.

Daarnaast is er een verdampingsmodel voor brandstoffen en emulsies met meerdere componenten geïmplementeerd in de Euler-Lagrange-vergelijking. Het model is geschikt voor de drukwaarden die kenmerkend zijn voor heavy-duty gasturbines en gaat uit van een verhoging van de verdampingssnelheid van de meest vluchtige component zodra de temperatuur van de druppeltjes het kookpunt bereikt. De verdamping van emulsiedruppeltjes wordt gemodelleerd volgens een schilbenadering waarbij ervan wordt uitgegaan dat het water in de kern van het druppeltje niet verdampt. Er is een diffusiviteitsmodel geïntroduceerd voor het transport van de dispersiefase van de kern naar de buitenste schil. Voor de validatie worden experimentele gegevens over druppeltjes n-heptaan en n-decaan bij verschillende omgevingstemperaturen gebruikt.

Voor het verbrandingsmodel is de "Flamelet Generated Manifold"-benadering gehanteerd. De mengselfractie wordt op basis van de fracties van de soortelijke massa's berekend en gekoppeld aan het verdampingsmodel. Er worden voor verschillende enthalpieën en mengselfracties "flamelet"-tabellen opgesteld zodat deze kunnen worden gebruikt in situaties waarin niet wordt voorgemengd. Het thermochemische model kan met behulp van 4D-tabellen voor maximaal twee brandstofcomponenten worden gebruikt. Eendimensionale resultaten van het verbrandingsmodel, beschreven in termen van laminaire 
vlamsnelheid en profielen van de hoofdsoorten, worden vergeleken met de tot in detail uitgewerkte chemische oplossingen voor mengsels van hexadecaan, water en lucht bij twee drukniveaus.

$\mathrm{Na}$ de afzonderlijke evaluatie van elk model worden er gekoppelde simulaties uitgevoerd om de interactie daartussen te analyseren en te verifiëren dat ze geschikt zijn voor gebruik in de simulatie van gedeeltelijk voorverdampte brandstofstraal vlammen. Verdampende brandstofstralen met verschillende momentverhoudingen bepalen de gevolgen van de straalprocesparameters op de hoeveelheid verdampte massa, terwijl eendimensionale brandstofstraal vlammen de invloed zichtbaar maken van de verstuivingsstatistieken op de positie van de vlam. Voor beide configuraties is ook onderzocht wat het effect is als er water wordt toegevoegd.

Er worden numerieke simulaties uitgevoerd van de verbrandingskamer uitgerust met een straalbuis bij verschillende brandstofmengsels, waaronder vloeibare brandstoffen. Als referentie zijn experimentele gegevens gebruikt die afkomstig zijn van metingen door het DLR Institute of Combustion Technology. Voor gasvormige brandstofmengsels worden de resultaten van de simulatie vergeleken met OH-chemoluminescentie-beelden en PIVmeetgegevens. Zowel de lengte van de vlam als de vlamstabilisatieafstand worden kwalitatief goed voorspeld en de verandering in vlamintensiteit bij een massapercentage van 0 tot $40 \%$ propaan wordt goed voorspeld. De snelheidsprofielen binnen de verbrandingskamer komen goed overeen met de experimenten. Voor vloeibare brandstoffen worden de simulaties kwalitatief vergeleken met $\mathrm{OH}$-chemoluminescentie en beelden van Mie-verstrooiing. Net als bij het experiment wordt de stroming van een kleine hoeveelheid niet-verdampte massa bij de ingang van de verbrandingskamer geobserveerd. In vergelijking met het gebruik van gas is de vlam stabieler en intenser. Toevoeging van water leidt tot tragere verdamping van de brandstofstraal, meer interactie tussen de straal en de wand, een lagere temperatuur in de gasfase en een minder intense vlam. Als de temperatuur van de toegevoerde lucht stijgt, neemt de verdampingssnelheid toe en is de vlam stabieler.

Er is een benadering op basis van een geforceerde respons gehanteerd, waarbij gebruik is gemaakt van monofrequente excitaties bij de grens van de inlaat om de akoestische respons van elk model afzonderlijk te bepalen. De verstuivings- en verdampingsprocessen worden door akoestische golven bevorderd doordat deze afhankelijk zijn van de relatieve snelheid. Een voorgemengde vlam wordt via de dikte van de vlam door de akoestiek beïnvloed. Er is ook forcering toegepast op gekoppelde simulaties, zodat de akoestische respons van verdampende én eendimensionale brandstofstraal vlammen kon worden beoordeeld. Vervolgens is de respons van de verbrander in de straalbuis bij gebruik van zowel vloeibare als gasvormige brandstof geanalyseerd. 


\section{Acknowledgements}

First of all, I would like to express my thanks to Dr. Jaap van Kampen and Dr. Jim Kok for giving me the opportunity to undertake this research as well as to Dr. Verena Klapdor for taking over the project. I am also thankful to Dr. Christan Beck and Peter Kaufmann for being my supervisors within Siemens. I appreciate the help and support that they all provided me in both management and technical aspects.

I want to express my gratitude to Bastian Werdelmann and Stefan Dederichs for their collaboration and for providing the base to start my research. I am also grateful to Pascal Gruhlke, Lukasz Panek, Arnold Herrgen, Sebastian Hermeth, Marco Losurdo, Vahid Sharifi, Günther Walz, Bastian Geissler, Matthias Hase, Jürgen Meisl, Michael Huth, Bertram Janus, Bern Prade and any other internal or external Siemens employee that has been involved in this project in more or less extent.

I also want to thank Michael Severin, Holger Ax, Oliver Lammel and Rainer Lückerath for arranging my visit to the DLR and for performing the jet flame experiments. Part of their work, in terms of measurement data, is used in this work.

I acknowledge as well the financial support received from the Marie Curie Actions and Siemens AG, without which this research would have not been possible.

I cannot forget to mention the research colleagues from the COPA-GT project. I enjoyed the regular meetings as well as the leisure activities that we carried out together.

To conclude, I want to thank my parents and relatives as well as my partner for their understanding and for standing by my side during this period. 


\section{Contents}

Abstract $\quad$ i

Samenvatting $\quad$ iii

Acknowledgements $\quad$ v

Contents $\quad$ ix

Nomenclature $\quad$ xiv

1 Introduction 1

1.1 Motivation and scope . . . . . . . . . . . . . . . . 1

1.2 Dissertation structure . . . . . . . . . . . . . . . . 6

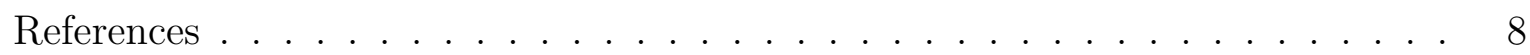

2 Atomization model $\quad 9$

2.1 Introduction and state of the art f . . . . . . . . . . . 9

2.2 Models . . . . . . . . . . . . . . . . . . . . . . . . . 11

2.2.1 Primary atomization model . . . . . . . . . . . . . . 11

2.2.2 GPIC Euler-Lagrangian coupling model . . . . . . . . . . . . . . . . 13

2.3 Results . . . . . . . . . . . . . . . . . . . 15

2.3.1 Calibration of the GPIC model . . . . . . . . . . . . . . . 15

2.3.2 Mesh dependency of the PIC and GPIC models . . . . . . . . . . 17

2.3.3 Validation . . . . . . . . . . . . . . . . . . . . 20

References . . . . . . . . . . . . . . . . . 25

3 Evaporation model $\quad 27$

3.1 Introduction and state of the art . . . . . . . . . . . 27

3.2 Model . . . . . . . . . . . . . . . . . . . . . . . . . . . 29

3.2.1 Major model adjustments . . . . . . . . . . . . . 30

3.3 Results . . . . . . . . . . . . . . . . . . . . 32

3.3.1 Single phase droplets . . . . . . . . . . . . . . . . . 32

3.3.2 Emulsion droplets . . . . . . . . . . . . . . . . . . . 35 


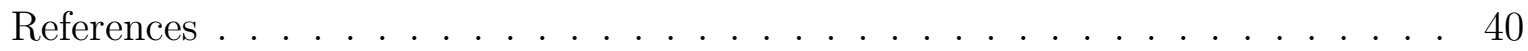

4 Combustion model $\quad 41$

4.1 Introduction and state of the art f . . . . . . . . . . . . . 41

4.2 Model formulation . . . . . . . . . . . . . . . . . . . . 42

4.2 .1 Table variables . . . . . . . . . . . . . . . . . . . . 42

4.2 .2 Transport equations . . . . . . . . . . . . . . . . 43

4.2.3 Calculation of product species mass fractions . . . . . . . . . . . . . 44

4.3 Results . . . . . . . . . . . . . . . . . . . . . 45

4.3.1 Effect of water content . . . . . . . . . . . . . . 46

4.3 .2 Effect of pressure . . . . . . . . . . . . . . . . 49

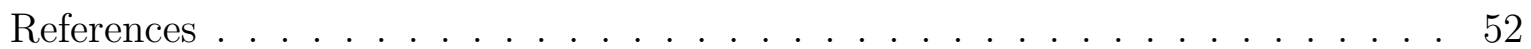

5 Models coupling $\quad 53$

5.1 Atomization-evaporation models coupling . . . . . . . . . . . . 53

5.1 .1 Introduction and setup . . . . . . . . . . . . . 53

5.1 .2 Results.......................... 54

5.2 Evaporation-combustion models coupling . . . . . . . . . . . 55

5.2 .1 Introduction and implementation method . . . . . . . . . . 55

5.2 .2 Verification . . . . . . . . . . . . . 57

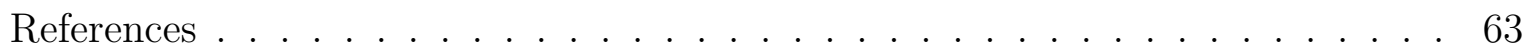

6 Models' validation for jet flames $\quad 65$

6.1 Introduction and literature review . . . . . . . . . . . . 65

6.2 Experimental setup . . . . . . . . . . . . . . . 66 66

6.3 Simulations setup . . . . . . . . . . . . . . . . 68

6.3 .1 Flamelet database . . . . . . . . . . . . . . . . 70

6.4 Results . . . . . . . . . . . . . . . . . . . . . . 72

6.4.1 Gas fuel . . . . . . . . . . . . . . . . . 72

6.4 .2 Liquid fuel . . . . . . . . . . . . . . . . . 81

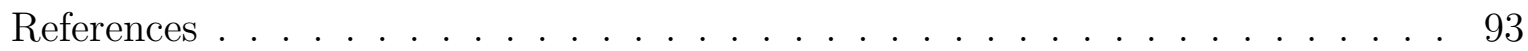

7 Numerical investigation of the models' acoustic response $\quad 95$

7.1 Introduction and literature review . . . . . . . . . . . . . . . 95

7.2 Approach . . . . . . . . . . . . . . . . 96

7.3 Results . . . . . . . . . . . . . . . . . . . 100

7.3.1 Atomization . . . . . . . . . . . . . . . 100

7.3.2 1-D droplet evaporation . . . . . . . . . . . . . . . 101

7.3.3 1-D combustion . . . . . . . . . . . . . . . . . 104

7.3.4 Evaporating spray . . . . . . . . . . . . . 105 
7.3 .5 1-D spray flame . . . . . . . . . . . . . . . 107

7.3 .6 Jet flame . . . . . . . . . . . . . . . . . . . . . . 109

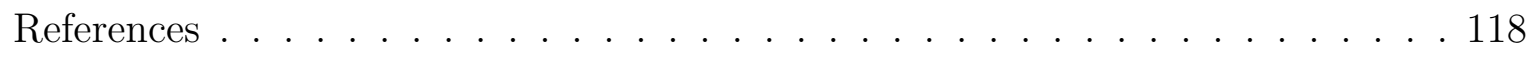

$\begin{array}{llr}8 & \text { Conclusions } & 119\end{array}$

$9 \begin{array}{lll}9 & \text { Recommendations } & 121\end{array}$

A Atomization of a liquid jet at 40 degree injection angle 123

B Evaporation of mono-component droplets under natural and forced convection

C Evaporation of heptane droplets under micro-gravity 129

D Combustion model formulation for bi-component fuels 131

$\begin{array}{ll}\text { E Simulation of the Siemens scaled can burner } & 137\end{array}$

F Jet flame simulation with the coarse grid and enthalpy clipping $\quad 145$ 


\section{Nomenclature}

\section{Symbols}

$\Delta t_{i n j}$ Elapsed time between the injection of two parcels

$\Delta x \quad$ Change in axial position from the previous sampling time

$\dot{m} \quad$ Mass flow

A Parameter of the Peng-Robinson equation of state

$B$ Parameter of the Peng-Robinson equation of state

$b \quad$ Molar fraction of Oxygen in air

$B_{m} \quad$ Mass transfer number

c Flame progress variable

$C_{D} \quad$ Drag coefficient

$C_{n} H_{m}$ Primary hydrocarbon species

$C_{p} \quad$ Heat capacity at constant pressure

$C_{p} H_{q}$ Secondary hydrocarbon species

$C_{c o l}$ Constant that determines the characteristic column breakup time scale

CO Carbon monoxide

$\mathrm{CO}_{2}$ Carbon dioxide

\section{Diffusivity}

d Droplet diameter

$d_{\text {circ }} \quad$ Diameter of a non-deformed parcel

$d_{\text {cross }}$ Major axis of an ellipse

$d_{\text {ref }}$ Diameter of a circle whose circumference is the same as the one of an ellipse

$d x \quad$ Axial cell size of a 1-D mesh

F $\quad$ Frequency

f $\quad$ Fugacity

$F_{t h} \quad$ Artificial flame thickening factor

$g \quad$ Gravity acceleration

Gr Grashof number

$h \quad$ Sum of the sensible and the chemical enthalpies

$H_{2} \quad$ Hydrogen

$\mathrm{H}_{2} \mathrm{O}$ Water

$K \quad$ Coefficient to account for the convective transport of the dispersed phase from the core to the shell

$k_{B} \quad$ Boltzmann constant

$L_{v} \quad$ Latent heat of vaporization

M Molecular weight 
$m \quad$ Number of hydrogen atoms in a molecule of the primary fuel species

$M_{d} \quad$ Droplet mass

$n \quad$ Number of carbon atoms in a molecule of the primary fuel species

$N_{2} \quad$ Nitrogen

NOx Nitrogen oxides

$n P \quad$ Number of particles represented by the parcel

$\mathrm{Nu} \quad$ Nusselt number

$\mathrm{O}_{2} \quad$ Oxygen

$P \quad$ Pressure

$p \quad$ Number of carbon atoms in a molecule of the secondary fuel species

$q \quad$ Number of hydrogen atoms in a molecule of the secondary fuel species

$r \quad$ Radius

Sc Schmidt number

Sh Sherwood number

Su Laminar flame speed

$S u^{\prime} \quad$ Flame speed calculated from the flow velocity at an iso-surface located upstream of the flame

T Temperature

$t \quad$ Time

$t^{*} \quad$ Elapsed time from injection for the column breakup event
U Velocity magnitude

V Volume

$W \quad$ Secondary species mass fraction to mixture fraction ratio

$x^{\prime} \quad$ Axial distance from the injection points

$Y \quad$ Mass fraction

Z Mixture fraction

$Z_{c} \quad$ Compressibility factor

\section{Greek symbols}

$\alpha \quad$ Thermal conductivity

$\beta$ Amount of moles of the $C O$ product species

$\chi \quad$ Molar fraction

$\dot{\omega} \quad$ Source term

$\epsilon \quad$ Amount of moles of the $\mathrm{CO}_{2}$ product species

$\eta \quad$ Amount of moles of the $\mathrm{H}_{2} \mathrm{O}$ product species

$\gamma \quad$ Activity coefficient

$\mu \quad$ Dynamic viscosity

$\nu \quad$ Amount of moles of the secondary fuel species

$\Omega \quad$ Liquid mass flow

$\Phi \quad$ Fugacity coefficient

$\phi \quad$ Equivalence ratio

$\psi \quad$ Generic variable or field

$\rho \quad$ Density

$\sigma \quad$ Surface tension 
$\tau \quad$ Time lag

$\tau_{r} \quad$ Relaxation time

$\theta$ Amount of moles of the $\mathrm{H}_{2}$ product species

$\Xi \quad$ Wrinkling coefficient

$\xi \quad$ Degree of parcel deformation

$\zeta \quad$ Amount of moles of the $\mathrm{O}_{2}$ product species

\section{Abbreviations}

AFR Air-to-fuel ratio

ATF Artificially Thickened Flame

BC Boundary conditions

CCD Charge-coupled device

CFD Computational Fluid Dynamics

CFL Courant Friedrichs Lewy number

CSP Computational Singular Perturbation

DLR Deutschen Zentrums für Luft- und Raumfahrt

DNS Direct Numerical Simulations

EBU Eddy break-up

FGM Flamelet generated manifold

GpC Ghosts per cell

GPIC Ghost Particles-in-cells

GTCC Gas Turbine Combined Cycle

GTCHP Gas Turbine Combined Heat and Power
HLB Hydrophilic-lipophilic balance

HPC High performance computing

IGCC Integrated coal Gasification Combined Cycle

LES Large Eddy Simulation

LHV Lower Heating Value

LODI Local One Dimensional Inviscid

MP-PIC Multiphase Particle-in-cell

Nd:YAG Neodymium-doped yttrium aluminium garnet

NRBC Non-reflecting boundary conditions

O/W Oil-in-water

OHCL OH-chemiluminescence

PDF Probability density function

PIC Particle-in-cell

PIV Particle image velocimetry

PM Particulate Matter

RANS Reynolds-Averaged Navier-Stokes

SCRS Simple chemical reacting system

SGS Sub-grid scale

SMD Sauter Mean Diameter

SRR Surface regression rate

W/O Water-in-oil

\section{Subscripts}

$0 \quad$ Initial injection conditions

$\infty \quad$ At the far field

a Air 
av Area-averaged

$b \quad$ Burnt mixture

boil At the boiling point

col Column breakup point

core In the core region of an emulsion droplet

d Droplet

eff Effective

$e q \quad$ Equilibrium conditions

ev Evaporation

exp Experiments

$f \quad$ Fuel

FC Forced convection

frac Fraction

$g \quad$ Gas phase

$h$, lagr Enthalpy transferred from the lagrangian parcels

$i \quad$ Each species of the droplet composition

in At the inlet boundary

inj Of the injected fuel or parcels

int Interaction with the ghost particles

j Species whose boiling temperature equals the droplet temperature

$l \quad$ Liquid phase lam Laminar flame

M, lagr Mass transferred from the lagrangian parcels

$n \quad$ Normal to the trajectory

$N C$ Natural convection

out At the outlet patch

prem Fully premixed state

rel Relative to the gas phase

rpv Reaction progress variable

$s \quad$ At the droplet surface

sampl Sampling interval

sat At the saturation pressure

sgs Sub-grid scale

sim Simulation

st Stoichiometric conditions

str From stripping

U, lagr Momentum transferred from the lagrangian parcels

$u b \quad$ Unburnt mixture

up Upstream the jet injection point

$w \quad$ Water species

$x \quad$ In the axial direction

$Y$, lagr Species mass fraction transferred from the lagrangian parcels 


\section{Chapter 1}

\section{Introduction}

\subsection{Motivation and scope}

Due to the steady growth of world population and the economic expansions, the demand in global energy increases yearly as shown in Figure 1.1. The most consumed source of energy is the oil, followed by the electricity and the natural gas. Thus, about two thirds of the total energy consumed is directly obtained from fossil fuels.

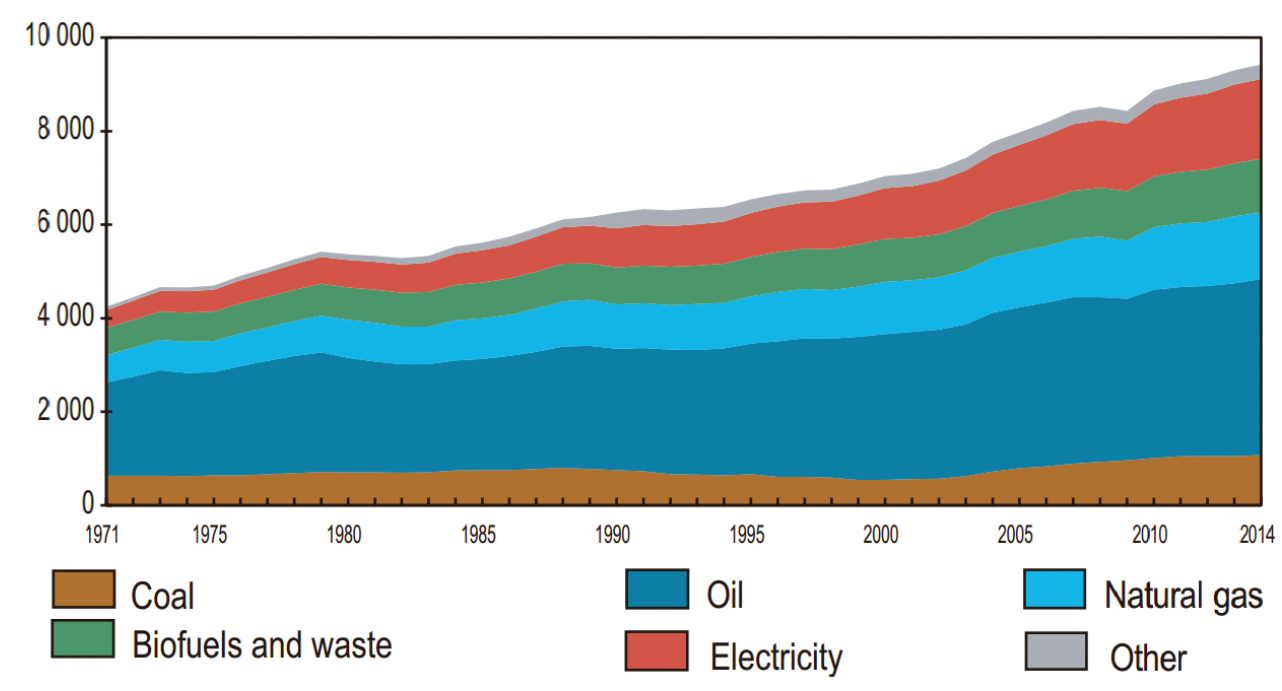

Figure 1.1: World total energy consumption, in million tones of oil equivalent, from 1971 to 2014 by source [1]. The solar, wind, geothermal, etc. energy sources are included in the "Other" group.

Figure 1.2 presents the shares in consumption of each resource by sectors. The coal is mainly used by the industry while most of the oil is employed in the transport sector. The latter is mainly caused by the high energy density of liquid fuels. The natural gas and electricity are predominantly consumed by both the industry and the agricultural/domestic/services sectors. In turn, the consumable electricity has to be generated from other energy sources. Figure 1.3 indicates that coal is the main source for power 


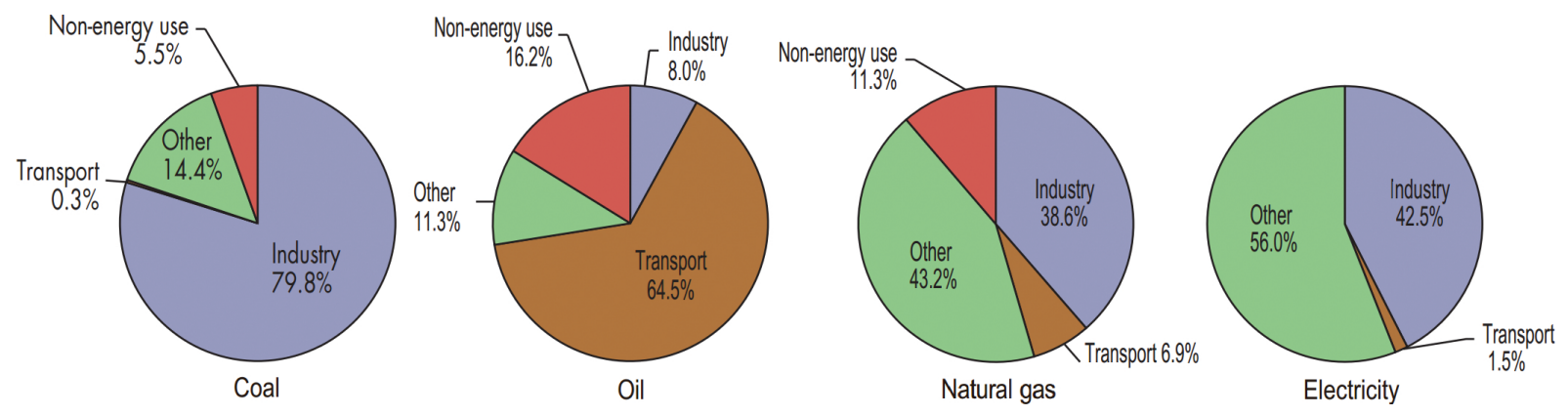

Figure 1.2: Shares of the world total consumption of the main energy sources in 2014 by sectors [1]. The agricultural, commercial, residential, etc. sectors are included in the "Other" group.

production with a $40 \%$ of the share, followed by the renewable, natural gas and nuclear energies with approximately $25 \%, 20 \%$ and $10 \%$ of the share respectively. Thus, only $5 \%$ of the electrical energy is produced from oil.

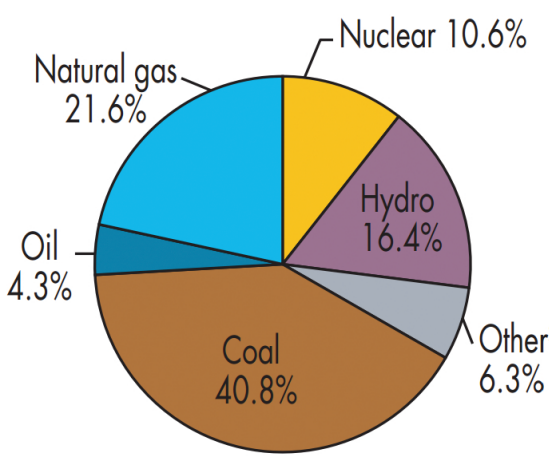

Figure 1.3: Shares of the world total electricity generated in 2014 by source [1]. The solar, wind, geothermal, etc. energy sources are included in the "Other" group.

The use of industrial gas turbines lie mainly within the electricity generation from the fossil fuels, although bio fuels can also be used. In the IGCC cycle, the coal is gasified and converted into raw syngas. After removing impurities such as particulate matter or sulfur, which require a cooling down stage after the gasification, the clean syngas is fed to the gas turbine[2]. Conversely, the natural gas and oil fuels do not require any pre-processing stage. The combined cycles aim to increase the thermal efficiency by recovering the sensible energy contained in the flu gases generated by the gas turbine. This is commonly done via cogeneration (GTCHP cycle) or by heating up water in a boiler to create steam at high pressure and temperature so that additional electrical energy can be generated in a steam turbine (GTCC cycle). Depending on the application and on the fuel used, the power than can be produced with gas turbines range from 4 to $400 \mathrm{MW}[3]$.

Apart of the increasing demands on efficiency, reliability, adaptability and fuel flexibility [4], gas turbines also have to meet more stringent ecological requirements. The typical emission levels generated using the different fuel types and power plant configurations are shown in Figure 1.4. The highest NOx, sulfur, $\mathrm{CO}_{2}$ and soot are produced with coal, 
but a substantial reduction is obtained with the gasification process in the IGCC cycle. Oil is the next fuel with the highest emission levels and it is followed by the natural gas, whose combustion does not produce any $\mathrm{PM}$ or $\mathrm{SO}_{2}$. The higher power produced with the combined cycles leads to a lower amount of emissions per energy unit, being the GTCHP cycle more efficient than the GTCC cycle. Additionally, vegetable oils can be a potential replacement of diesel in the future due to its lower emissions level.
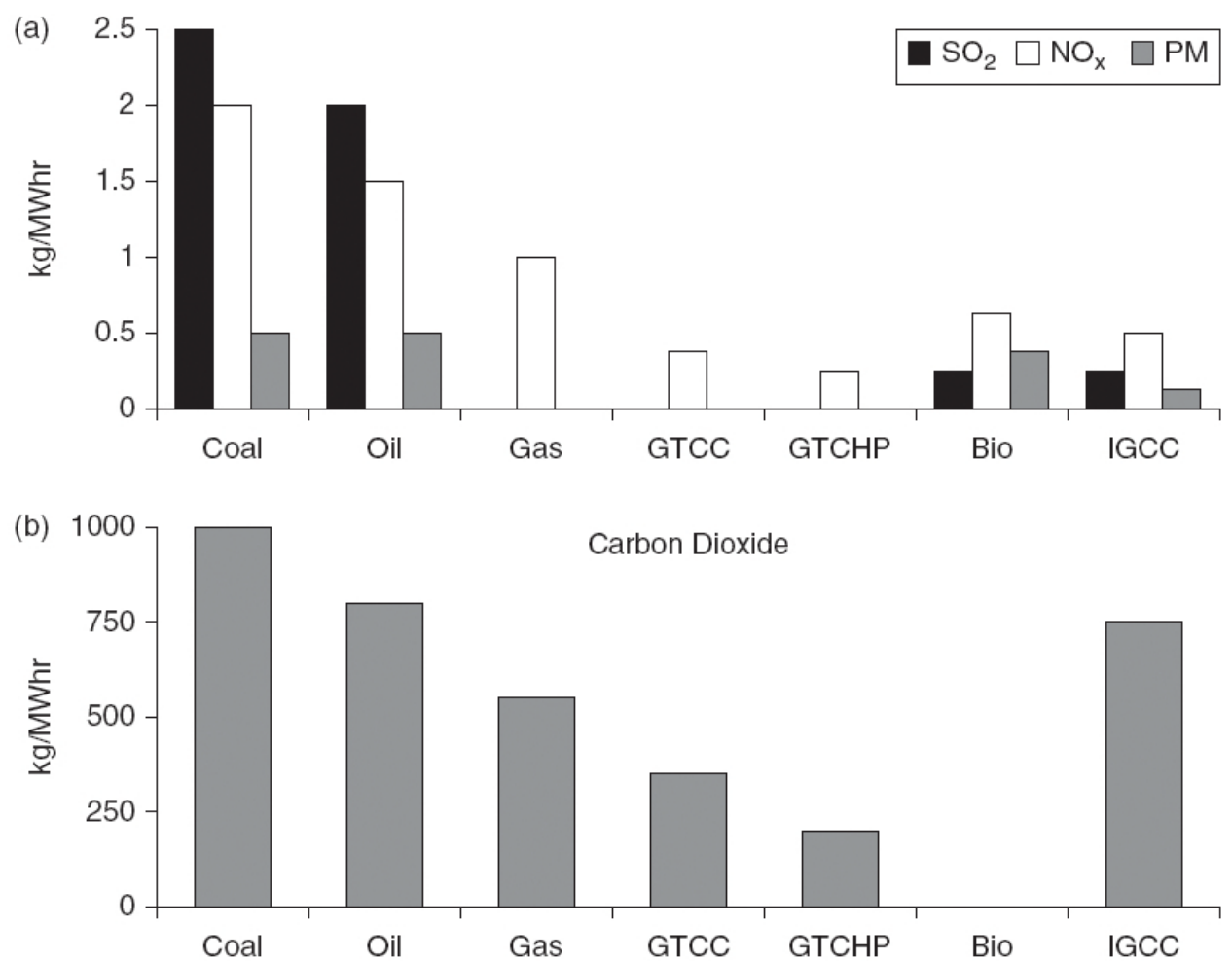

Figure 1.4: Comparison of the emission levels in gas turbines with different fuels and thermodynamic cycles [5]

Due to the lower emissions level of natural gas, it is the fuel predominantly used in heavy-duty gas turbine engines. However, oil is usually used as a back-up fuel so that the engine can operate in case there is a gas fuel cut-off. Furthermore, in regions where there is large oil production - which typically results in low prices - or the gas resources are limited, the capability to operate the engine with oil fuel at low emission levels becomes more important.

The reduction of pollutants is technically challenging and becomes a major concern due to its impact on the environment as well as on the human health. Therefore, it is crucial to develop combustion systems that comply with the ecological demands and regulations. Particularly, $\mathrm{CO}_{2}$ and $\mathrm{NOx}$ reduction are currently the main priorities in gas turbine combustion [5]. The former is achieved by increasing the thermal efficiency of the system (e.g. combined cycles) while the latter is accomplished with the lean premixed combustion systems, which are predominant in modern heavy-duty gas turbines nowadays [6]. Lean operation leads to low flame temperatures, and therefore to low NOx production, but it 
has several drawbacks like flame quenching or thermo-acoustics.

Compared to gas operation, the use of liquid fuels involves several and complex spray processes that take place within the combustion chamber and affect the fuel distribution. The latter has an influence on the temperature distribution and, therefore, on the emissions level. In general, dry oil operation leads to a higher amount of nitrogen oxides than gas operation because of the lower fuel air-mixing. The latter is caused by the additional time required to atomize and vaporize the fuel. However, the residence time cannot be increased either (for better mixing) due to the auto-ignition phenomena, specially at high pressures, which can lead to flashback and consequently to a potential engine damage. Thus, oil flames are typically non-premixed. This implies that there are regions with locally high temperatures which lead to a higher NOx production than at fully premixed conditions.

Figure 1.5 illustrates the evolution of the $\mathrm{CO}$ and NOx with the engine load for nonpremixed flames. The $\mathrm{CO}$ level is high at partload and it reduces progressively with the load until it falls below the limit. On the other hand, NOx emissions are usually above the limitations, even at partload, and they tend to increase with higher flame temperatures. The addition of water to the liquid fuel aims to reduce the flame temperature, and therefore the NOx level, by decreasing the temperature upstream the flame (larger amount of heat absorbed during the evaporation process) as well as increasing the specific heat capacity of the unburnt mixture. Additionally, the soot generated at the flame is also reduced [7]. However, the flame stability gets affected and the spray processes become even more complex. Thus, for the combustor design it is important to understand the phenomena involved in liquid fuel combustion and the way they interact between each other.

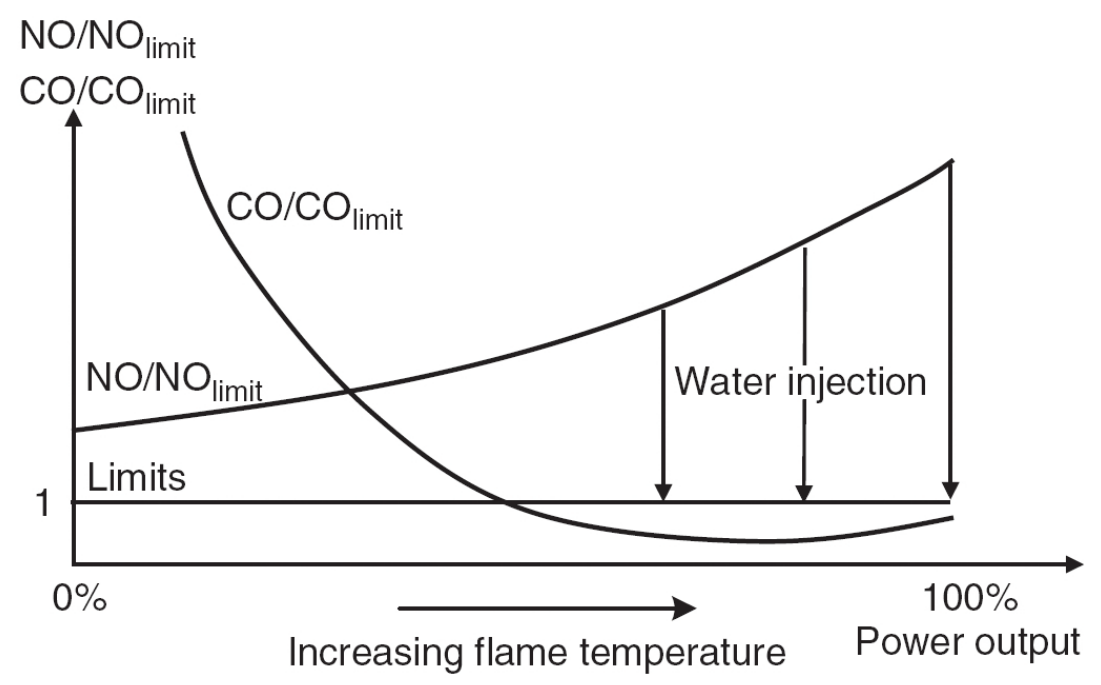

Figure 1.5: Sketch of the CO and NOx curves for non-premixed flames with the load [5]

The high cost of experimental tests as well as the limited measurement techniques and data post-processing motivates the development of numerical methods that can accurately 
predict the behaviour of the system. This dissertation focuses on the development of a tool that supports the design and optimization of fuel oil systems. It is a compressible CFD solver that predicts the flow field inside a certain domain, which can be defined by any geometrical feature, from the conditions imposed at its boundaries. Due to the flexibility in the model implementation, the OpenFOAM software is used as the base CFD code for the tool development. The aim of this tool is simulate the combustion process within a gas turbine using liquid fuels, particularly water-in-oil emulsions. For this, models are developed within the context of Large-Eddy Simulation formulation as a compromise between the accuracy of DNS and the low computational cost of RANS. These simulations can provide an insight of the flow behaviour to better comprehend the operation of the combustion system at certain conditions.

Within the scope of this thesis, only liquid break-up and evaporation processes are considered. Consequently, it is assumed that other spray phenomena like cavitation, coking, micro-explosion or droplet-wall interaction do not have a big impact on the solution. Based on the liquid and air properties, the aim of the atomization model is to provide an adequate droplet distribution in terms of position and size. In turn, the evaporation model should predict the rate of mass transfer from the liquid to the gas phase. Apart from the gas phase properties, the evaporation rate of a droplet depends mainly on its size and its temperature. Since heat is required for the phase change, the amount of mass vaporized determines the evolution of the droplet temperature. Within a gas turbine, the heat transferred from the air - which has been heated-up in the compressor - usually exceeds the latent heat of vaporization and the temperature of the droplets increase. In the case that the temperature difference is not large enough, the remaining heat would be extracted from the liquid phase and the droplet temperature would decrease. Regarding the combustion model, it assumes that the flame is thin enough so that the local structure within the flame front corresponds to the one from a laminar flamelet. The latter is sensitive to local changes in the equivalence ratio and enthalpy. The instantaneous shape of the flame front is affected by turbulence, which tends to wrinkle the flame and therefore increase the effective flame surface.

From the stated above, there is a direct coupling between the atomization-evaporation as well as the evaporation-combustion models. This is known as the fuel-oil modelling chain and it implies that any variation in either atomization or evaporation statistics affects, indirectly or directly, the flame. Therefore, errors caused by modelling inaccuracies, assumptions, gaps or uncertainties increase and accumulate along the chain. Thus, it is important to have a good prediction level in each individual model considered.

Furthermore, lean premixed combustion systems are prone to unsteady heat release that can trigger thermo-acoustic instabilities $[8,9]$. This results in noise and vibrations, which could induce severe damage to the gas turbine engine. For this reason, studies on the flame dynamics are essential for advanced engine development. A sophisticated method 


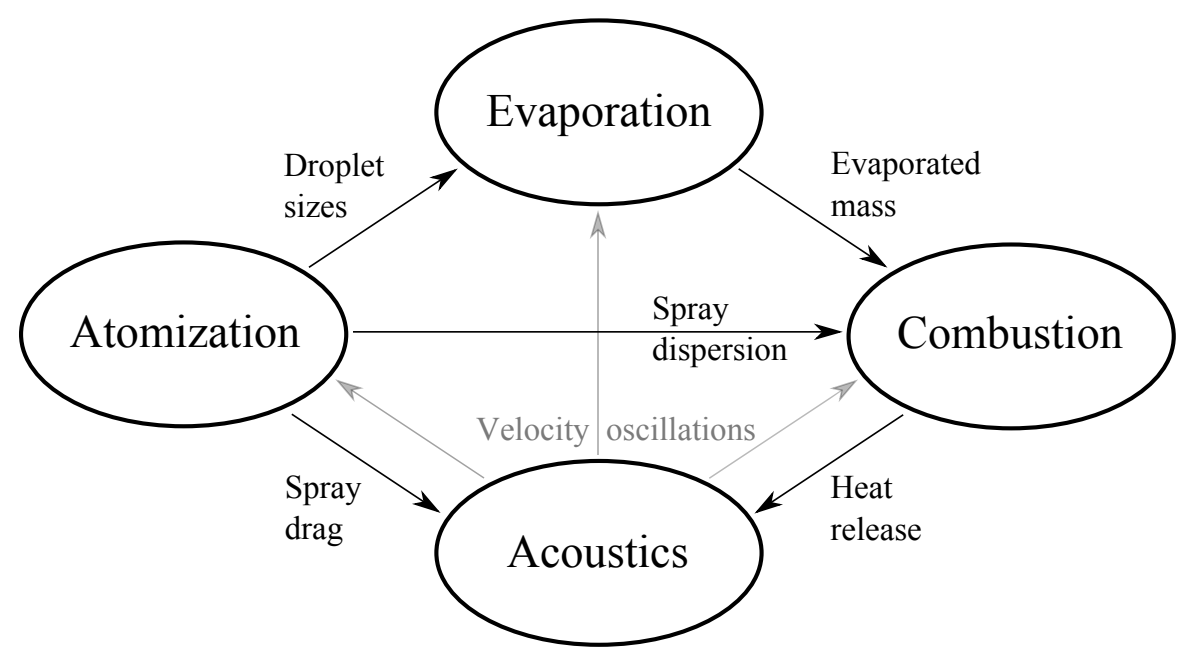

Figure 1.6: Acoustic feed-back loop for liquid fuel combustion

to analyse the thermo-acoustic stability is the forced response approach. The flame is excited artificially with velocity perturbations and the response is used to determine the Flame Transfer Function [10, 11]. Transfer functions describe the system's dynamics and can be used to control instabilities.

Large Eddy Simulations are appropriate to predict combustion instabilities [12]. In addition, the use of a compressible flow solver allows the simulation of acoustics waves in the computational domain. For gas operation, the acoustic waves influence the fuel distribution near the injection zone. Similarly, for oil operation the pressure and velocity waves affect the spray motion as well as the atomization and the evaporation processes. As explained above, this also causes equivalence ratio as well as enthalpy fluctuations at the flame front. Besides, acoustics also modify the flame position so that the fuel consumption rate changes. The latter effect is predominant in technically premixed systems. All the previous processes lead to heat release fluctuations that in turn affect the acoustic field. This phenomena is also known as the acoustic feed-back loop, which is illustrated in Figure 1.6 for oil operation. The influence of acoustic waves on the fuel supply processes affects indirectly the acoustic field after a certain time lag. Moreover, spray drag tends to damp acoustic oscillations.

The main objective of the present dissertation is to develop each of these models individually, to couple them and to evaluate the influence of acoustic waves on the whole modelling chain. The outcome of this work can be used to simulate more complex configurations and to develop correlations for low order tools.

\subsection{Dissertation structure}

This dissertation is divided in six main chapters. The present chapter is an general introduction to the topics that are investigated. In Chapter 2 the atomization model, combined 
with a novel coupling model between the liquid and gas phases, is presented. Simulations are performed for calibration and validation of the model parameters. The evaporation model for multi-component droplets is introduced in Chapter 3. Validation cases for mono-component, bi-component and emulsion droplets under different ambient conditions are simulated. The formulation of the combustion model is described in Chapter 4, where the laminar flame profiles and flame speeds are also compared with the detailed solution. The coupling between the models is analysed with the simulation of evaporating sprays in cross flows and 1-D spray flames in Chapter 5. Following this, a practical jet tube combustor is simulated with both gaseous and liquid fuels, where the three models are used, in Chapter 6. Results are compared to experimental data in terms of velocity distribution, flame shape and intensity, or amount of liquid phase within the combustion chamber. The acoustic response to forced excitations is investigated in Chapter 7 for each model individually as well as for the coupled configurations: an evaporating spray, a 1-D spray flame and the jet tube combustor case. Further validation cases and numerical analyses are included in the Appendixes A-F.

\section{References}

[1] International Energy Agency. Key world energy statistics. http://www . iea.org/ publications/freepublications/publication/KeyWorld2016.pdf.

[2] O. Maurstad. An Overview of Coal based Integrated Gasification Combined Cycle (IGCC) Technology. Tech. rep. Massachussets Institute of Technology, 2005.

[3] http : / / www . energy . siemens . com / ru / pool / hq / power-generation / gas turbines/downloads/gas-turbines-siemens.pdf. Accessed on 7.11.2016.

[4] B. Schmalzer. https : / / www . turbomachinerymag . com/gas-turbines-facingincreasing-demand. Accessed on 7.11.2016.

[5] T. C. Lieuwen and V. Yang. Gas Turbine Emissions. Cambridge University Press, 2013.

[6] S. A. Greenwood. "Low emission combustion technology for stationary gas turbine engines". In: Proceedings of the 29th Turbomachinery Symposium, Texas. 2007.

[7] F. L. Dryer. "Water addition to practical combustion systems - Concepts and applications". In: Symposium (International) on Combustion 16.1 (1977), pp. 279295.

[8] S. Seo. "Combustion instability mechanism of a lean premixed gas turbine combustor". In: KSME International Journal 17 (2003), pp. 906-913.

[9] T. C. Lieuwen. Unsteady Combustor Physics. Cambridge University Press, 2012. 
[10] H. J. Krediet. "Prediction of limit cycle pressure oscillations in gas turbine combustion systems using the flame describing function". PhD thesis. University of Twente, 2012 .

[11] S. Wagner et al. High Performance Computing in Science and Engineering. Springer, 2009 .

[12] T. Poinsot and D. Veynante. Theoretical and Numerical Combustion. R. T. Edwards, 2005 . 


\section{Chapter 2}

\section{Atomization model}

\subsection{Introduction and state of the art}

In order to simulate a spray and analyse its statistics, in terms of droplets' position and size, the well-known Euler-Lagrange approach is applied. It uses the Particle-inCell method that was initially developed by Harlow [1, 2]. The gas phase is computed by means of a finite volume method and the liquid phase is statistically represented by Lagrangian parcels. Each computational parcel represents a group of particles with similar geometrical and physical properties [3]. The properties of the surrounding gas field are interpolated onto the parcel position and the parcel's properties are evolved through suitable sub-models based on physical processes. For the parcel's motion, considering only aerodynamic and gravity forces, Equation (2.1) is solved:

$$
\frac{d^{2} x_{d}}{d t^{2}}=g+\frac{U_{r e l}}{\tau_{r, d}}
$$

The relaxation time is defined as follows:

$$
\tau_{d}=\frac{4}{3} \frac{\rho_{d} d}{\rho_{g} C_{D}\left|U_{r e l}\right|}
$$

where the drag coefficient depends on the geometrical shape of the parcel and on the Reynolds number:

$$
R e_{d}=\frac{\rho_{g}\left|U_{r e l}\right| d}{\mu_{g}}
$$

With the above equations the trajectory of a particle can be tracked. As the gas phase properties change from cell to cell, each time the parcel crosses a face the properties are interpolated again and the models are evolved [3]. Thus, the time step used for the Lagrangian particles is usually smaller than the time step employed in the computation of the gas phase fields. 
The motion of a particle is caused by the momentum transferred from the gas phase, which in turn slows down. If this momentum loss is not accounted in the simulation, there is a "one-way" coupling between the gas and the spray. For a "two-way" coupling, a momentum source term is included in the gas phase equation. Additionally, "four-way" coupled simulations also account for the interaction between parcels. However, due to the additional computational effort, only the "two-way" coupling is considered within the scope of this thesis. Considering a cell $k$ that has been crossed by $N$ parcels, the source term is calculated as the sum of the momentum change of those parcels at that cell:

$$
S p_{k}=\frac{1}{V_{k}} \sum_{i=1}^{N}\left(n P_{i} M_{i}\left(U_{i, \text { incell }}-U_{i, \text { outcellk }}\right)\right)
$$

Equation (2.4) indicates that the source term is inversely proportional to the cell volume. On the other side, the momentum transferred also depends on the size of the particles. In the case that a particle volume is similar or larger than the cell volume, a high momentum source term is transferred so that numerical instabilities or non-physical results are obtained. Thus, with the PIC model a low volume fraction is required. The same applies to the MP-PIC method, developed by Andrews and O'Rourke [4], despite it is an extension of the PIC model that accounts for the volume occupied by the parcels in the gas phase. The effect of the Euler-Lagrange coupling, in terms of gas phase interpolation and source term distribution, has been reported by Krista [5].

To avoid this limitation, several source term distribution methods are available in the literature. They consist in the transfer of the source terms to the vertices of the cell where the parcel is located. Depending on the method, different weighting factors are associated to the nodes:

- Lagrangian polynomials [5]: Lagrangian polynomials are used to transform the cell and the parcel's position from global coordinates to a local master cell. The shape functions at the local particle coordinates are used as the weigths for the distribution.

- volume-weighting [6]: the cell is divided in two parts per direction based on the parcel's location. Source terms are distributed based on the volume of the sub-cell closest to each node.

- distance-weighing [7]: the weighting factor is determined by the distance from the parcel to the cell vertices.

- CLE method [8]: A "gaseous" particle, which is attached to the parcel, is used to retain the source terms and release them gradually. Sources are transferred to a cell when the mean distance of the particle to the cell vertices is smaller than the radius of the "gaseous" particle. This radius increases with evaporation and reduces when source terms are released. 
Although the above models are more robust than the PIC model, the source term distribution is still very dependent on the mesh and problems can still arise for very high momentum transfers.

In the case of liquid sprays, models for atomization and breakup can also be used. The advantage of using physical models, rather than injecting a high amount of particles with a defined droplet size distribution, is that the effect of turbulence or acoustics on the atomization process is better captured. Additionally, once the models are calibrated, they are more reliable at operating conditions where no experimental data is available. For the simulation of sprays in cross flow, several models (TAB [9], Pilch-Erdman [10], Reitz-Diwakar [11], Kevin-Helmholtz/Rayleigh-Taylor [12], SHF [13]) were internally evaluated at Siemens and the results indicated that they were not suitable for the primary atomization process but for the secondary droplet break-up.

In this chapter, a novel model for the Euler-Lagrangian coupling for parcels with similar or larger volume than the cell volume is presented. This model is used together with a primary atomization and a secondary break-up models for the simulation of sprays in cross flow.

\subsection{Models}

\subsubsection{Primary atomization model}

The primary atomization of a liquid jet in cross flow, which results from a plain jet atomizer, is modelled according to Rachner et al. [14]. A sketch of the model concept is depicted in Figure 2.1. For a $90^{\circ}$ injection, the momentum ratio between the liquid jet and the cross flow - which correlates with the spray penetration - is defined in Equation (2.5).

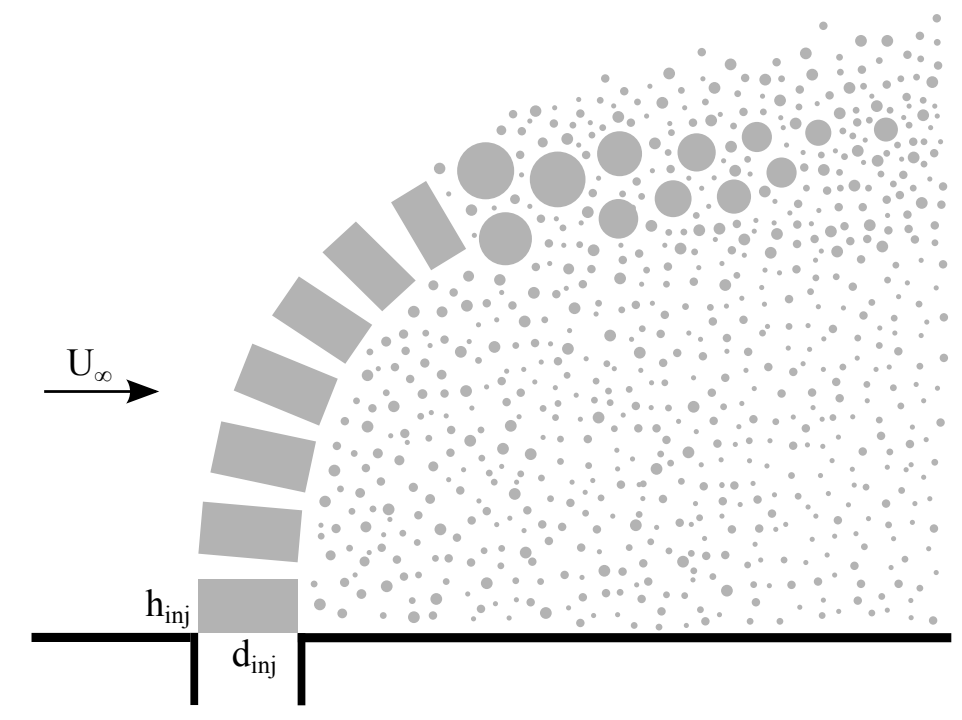

Figure 2.1: Primary atomization model of a jet in cross flow. 


$$
q=\frac{\rho_{l} U_{i n j}^{2}}{\rho_{g} U_{g}^{2}}
$$

Cylindrical droplets are introduced into the domain with the same diameter as the injector hole. The height parameter defines the amount of parcels used to discretize the liquid column, and therefore the resolution of the complete spray, and it is determined from the injection rate:

$$
h_{i n j}=U_{i n j} \Delta t_{i n j}=\frac{4 \dot{m}_{i n j} \Delta t_{i n j}}{\rho_{l} \pi d_{i n j}^{2}}
$$

The disintegration of the jet is governed by two breakup mechanisms: shear breakup and column breakup. In the shear breakup, droplets are shed from the jet surface due to the shear forces induced by the cross flow. The stripping rate is calculated with the equation below, which is derived from the boundary layer theory:

$$
\dot{m}_{s t r}=\frac{1}{2} \rho_{l} A \xi U_{r e l, n} \alpha_{l} \frac{\sqrt{\pi d_{r e f}}}{\pi d_{c i r c}}\left(1-\exp ^{-\frac{d_{\text {cross }}}{\alpha_{l} \sqrt{\pi d_{r e f}}}}\right)
$$

where:

$$
\begin{gathered}
A=\sqrt[3]{\frac{\rho_{g} \mu_{g}}{\rho_{l} \mu_{l}}} \\
\alpha_{l}=\sqrt{\frac{4 \mu_{l}}{\rho_{l} \xi U_{r e l, n} A}}
\end{gathered}
$$

Equation (2.7) accounts for the deformation of the injected cylindrical particles into an elliptical cylinder by means of the parameter $\xi$, which is the ratio between the major and minor semi-axis of an ellipse. This deformation has an impact on the drag coefficient and therefore on the jet trajectory. The size of the stripped droplets, which are modelled as spherical, is correlated with the boundary layer displacement thickness at the jet surface:

$$
\frac{\delta}{d_{s t r}}=\sqrt{\frac{\pi}{2}} \text { Oh } \sqrt[3]{\frac{\mu_{g}}{\mu_{l}}} \sqrt[6]{\frac{\rho_{l}}{\rho_{g}}} \sqrt{\frac{R e_{r e l}}{W e_{r e l}}}
$$

The column breakup results from the development of surface waves along the jet axis, which leads to the formation of primary droplets. The column breakup occurs when the elapsed time from injection reaches a characteristic time:

$$
t^{*}=C_{c o l} \frac{d_{0}}{U_{r e l, 0}} \sqrt{\frac{\rho_{l, 0}}{\rho_{g}}}
$$

The $C_{c o l}$ model parameter is set to 2.8 for jets injected perpendicularly into the cross flow, assuming a zero gradient of the velocity component normal to the injector wall. The primary droplets created at the breakup point are modelled as ellipsoids. They are defined from a spherical diameter and a deformation, from which their mass is calculated. 
The diameter is set to $30 \%$ of the injector hole diameter. The amount of primary droplets created depends on the mass of the cylindrical jet parcel at the column breakup point, which in turn is determined from the injection and stripping rate. If they are large enough so that a cylindrical parcels at the breakup point does not have enough mass to create at least one primary parcel with this diameter, only one primary parcel is created with the available mass. The deformation of these droplets is taken as the one of the elliptical cylindrical parcel, from which they are created, to the power of $(2 / 3)$ due to the additional dimension in which the parcel can deform. Shear breakup is also applied to the primary parcels until the aerodynamic Weber number falls below a critical value of 12 , based also on the work of Guildenbecher et al. [15].

The Kevin-Helmholtz/Rayleigh-Taylor hybrid model from Beale and Reitz [12] is selected to further atomize the parcels created from the shear breakup model. The RayleighTaylor breakup model predicts the growth of waves on the droplet surface. It is initiated when the droplet diameter is larger than the wave length of the fastest growing wave if the time of the disturbance growth is larger than the characteristic breakup time. On the other hand, the Kelvin-Helmholtz mechanism assumes that the droplet behaves like a liquid jet with perturbation waves growing on its surface and inducing mass stripping. It is then characterized by the breakup time using the fastest growing wave length and the aerodynamic Weber number.

\subsubsection{GPIC Euler-Lagrangian coupling model}

In Large-Eddy simulations of turbulent flows, the turbulence level resolved is determined by the computational mesh. While turbulent scales larger than the local cell size are solved by the filtered Navier-Stokes equations, smaller scales have to be modelled. Thus, a finer grid always leads to a more accurate solution since more scales are resolved. For the simulation of sprays, as aforementioned, the PIC model requires a low droplet-cell volume fraction.

With the primary atomization model presented in the previous section, where the jet parcels have a similar diameter as the injector hole, this limitation may be in conflict with the resolution of small turbulent scales. In order to avoid the volume fraction limitation, the source term of the jet and primary parcels (which can have a diameter similar to or larger than the cell size) should be distributed not only to the cell where they are located, but also to the neighbouring cells. The PIC coupling is applied to the secondary droplets due to their small size - typically lower than $0.1 \mathrm{~mm}$ - and the potentially large amount of these parcels in a spray simulation. Thus, different cloud types are assigned to the jet, the primary and the secondary parcels.

As illustrated on the left side of Figure 2.2, the Ghost-Particles-In-Cells coupling model consists in the placement of SPH ghost particles at the cells that surround the jet 
or the primary parcels. These ghost particles are able to interact with the spray parcels. They are used to interpolate the gas phase properties, which are utilized by the droplet sub-models, and to distribute the calculated source terms using using a kernel function $\beta$. In the present work, the kernel function chosen is a quintic polynomial equation.

In order to optimize the amount of particles required, each parcel does not have its own ghost particles. Conversely, as shown in Figure 2.2, a grid of ghost particles are created around the parcels and they are shared. To create this grid, an overall bounding box for the ghost parcels is defined by enlarging the overall spray cloud bounding box with the interaction radius. Ghosts particles are placed inside this box based on the Ghosts-perCell parameter, which defines the distance between them. For this, the minimum size of the cells where the spray parcels are located is taken. Once the box of ghost particles is determined, an iterative procedure - by means of the bisection method - is performed to remove the ghost parcels that are not within the local bounding box of any spray parcel. This procedure is performed every time step to adapt to the change of the spray parcels' position.
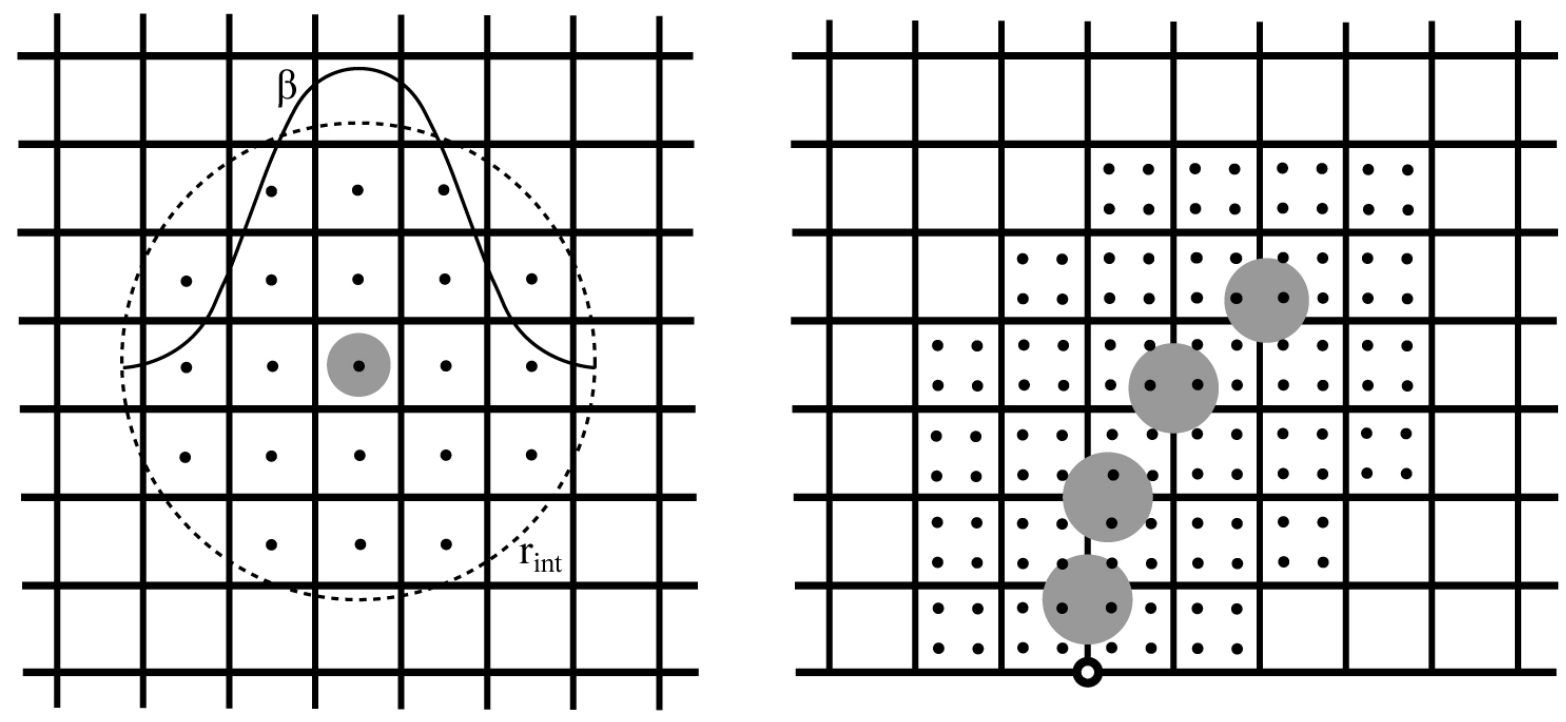

Figure 2.2: Sketch of the GPIC coupling model. Source terms are distributed to the SPH particles placed around it (left). They are placed in a structured way so that they are shared by different parcels (right). The black circumference represents the injection point.

For parallel computations, depending on the decomposition method, some ghost particles that are within the influence radius of a spray parcel can be located in a different partition. To solve this, virtual SPH particles are created at the same position by the processor containing the spray parcel. This virtual cloud can interact (i.e. exchange information) with the ghost cloud. Thus, information is passed from the ghost particles for interpolation and vice versa for source term distribution.

Further information on the SPH formulation can be found in the work of Gingold and Monaghan[16] as well as Violeau [17]. Additionally, details on the implementation of SPH method in OpenFOAM are described by Werdelmann et al.[18]. 


\section{$2.3 \quad$ Results}

The geometry used in the simulations is a $355 \times 25 \times 40 \mathrm{~mm}^{3}$ box, whose axial cross section matches the dimensions of the case employed in Rachner et al. [14]. Figure 2.3 displays the baseline mesh, which is used for the calibration of the coupling model. It has been generated from a uniform mesh, with a cell size of $1 \mathrm{~mm}$, by applying local refinements around the injection point. A mesh study is performed for both the ghost and PIC coupling models by removing the refinement regions. For these studies, URANS simulations are performed at the baseline conditions from Rachner et al. [14], using kerosene jet A-1 as a fuel and an initial liquid-to-air momentum ratio of 6 . The jet parcels are injected with a diameter of $0.45 \mathrm{~mm}$ and a temperature of approximately $293 \mathrm{~K}$. In most of the cases, the inlet air temperature is the same and the spray heat-up and vaporization processes can be neglected. Subsequently, LES simulations are performed - using the baseline mesh - for the validation of the combined primary atomization, secondary break-up and coupling models at several operating conditions. The "oneEqEddy" is used to model the sub-grid scale turbulence. Since the complete test geometry is not simulated, the turbulence generator method developed by Klein et al. [19] is used at the inlet patch. The inlet turbulence intensity is selected to match the intensity level at the injection point, estimated to be around 3\% [14], while the characteristic size of the introduced eddies is set to $5 \mathrm{~mm}$. Volume flux and SMD distributions are measured in the experiments at a plane $80 \mathrm{~mm}$ downstream of the injection point. In the CFD, a plane discretized in $1 \mathrm{~mm}^{2}$ squared faces is placed at that position. When a parcel crosses this plane, its properties are recorded so that the spray statistics can be processed. The time-averaging period used for post-processing is $10 \mathrm{~ms}$ for the URANS and $25 \mathrm{~ms}$ for the LES.

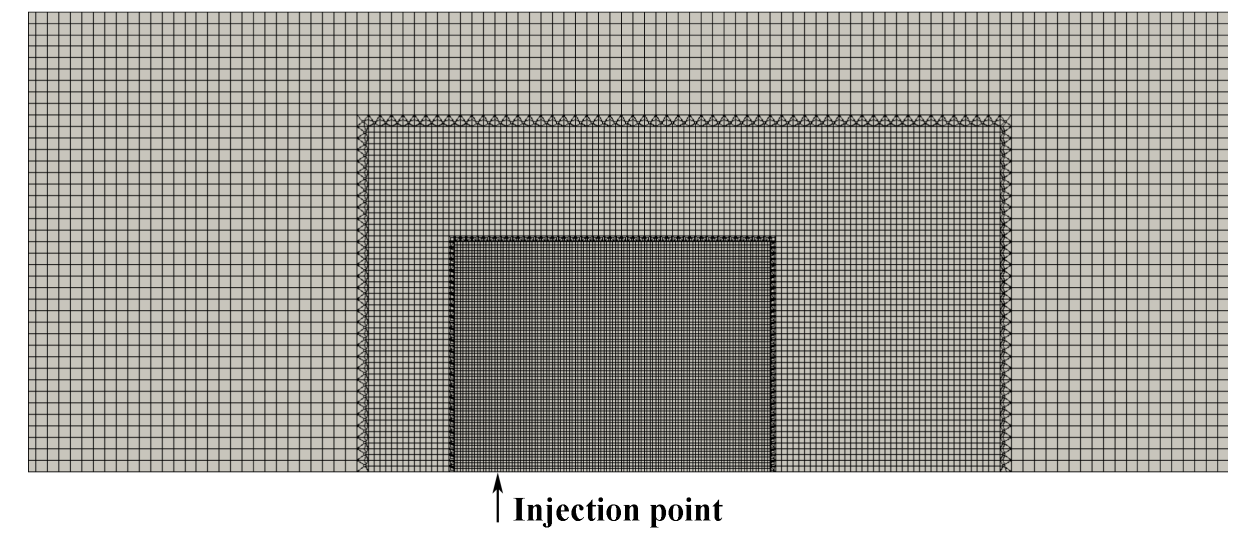

Figure 2.3: Refinement levels around the injection point.

\subsubsection{Calibration of the GPIC model}

Once a distribution function $(\beta)$ is selected, the two key parameter of the GPIC coupling model have to be determined for future simulations. The first one is the amount of ghost 

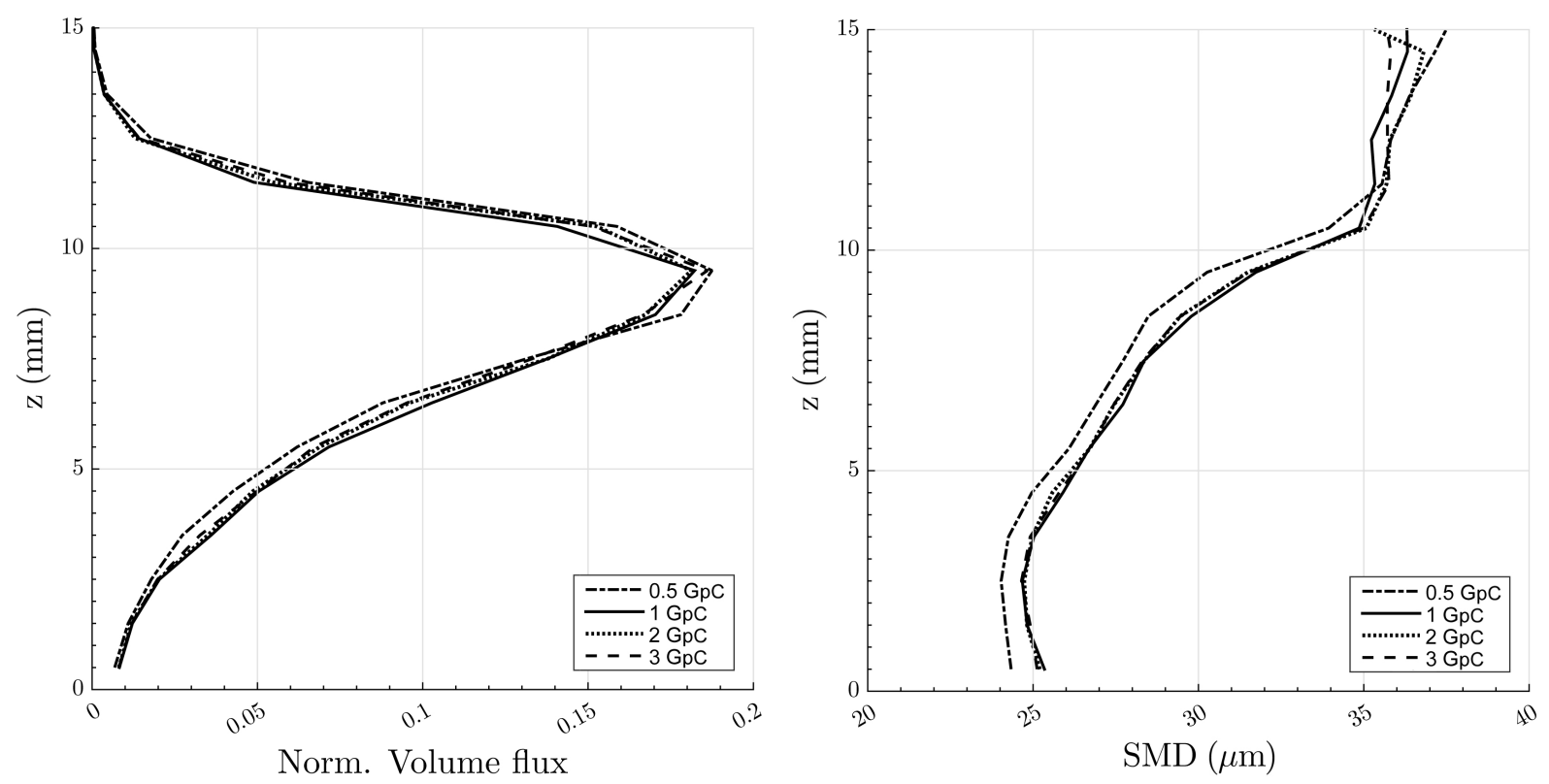

Figure 2.4: Effect of the amount of ghost particles per cell on the flux and SMD profiles.

parcels that are distributed to each cell and the second one is the distribution distance from the parcel center. The later is non-dimensionalized with the droplet radius.

Firstly, with a non-dimensional distribution distance of 3 , simulations are performed using from 0.5 (i.e. 1 parcel for every two cells) to 3 ghost parcels per cell and results are plotted in Figure 2.4. It can be observed that differences between the cases in both the depth-integrated flux and SMD profiles are not significant, especially between the cases with one or more ghost parcels per cell, so the dependency of the model to this parameter is low. As the computational effort increases with the amount of ghost parcels, it is considered that the use of one ghost parcel per cell is sufficient.
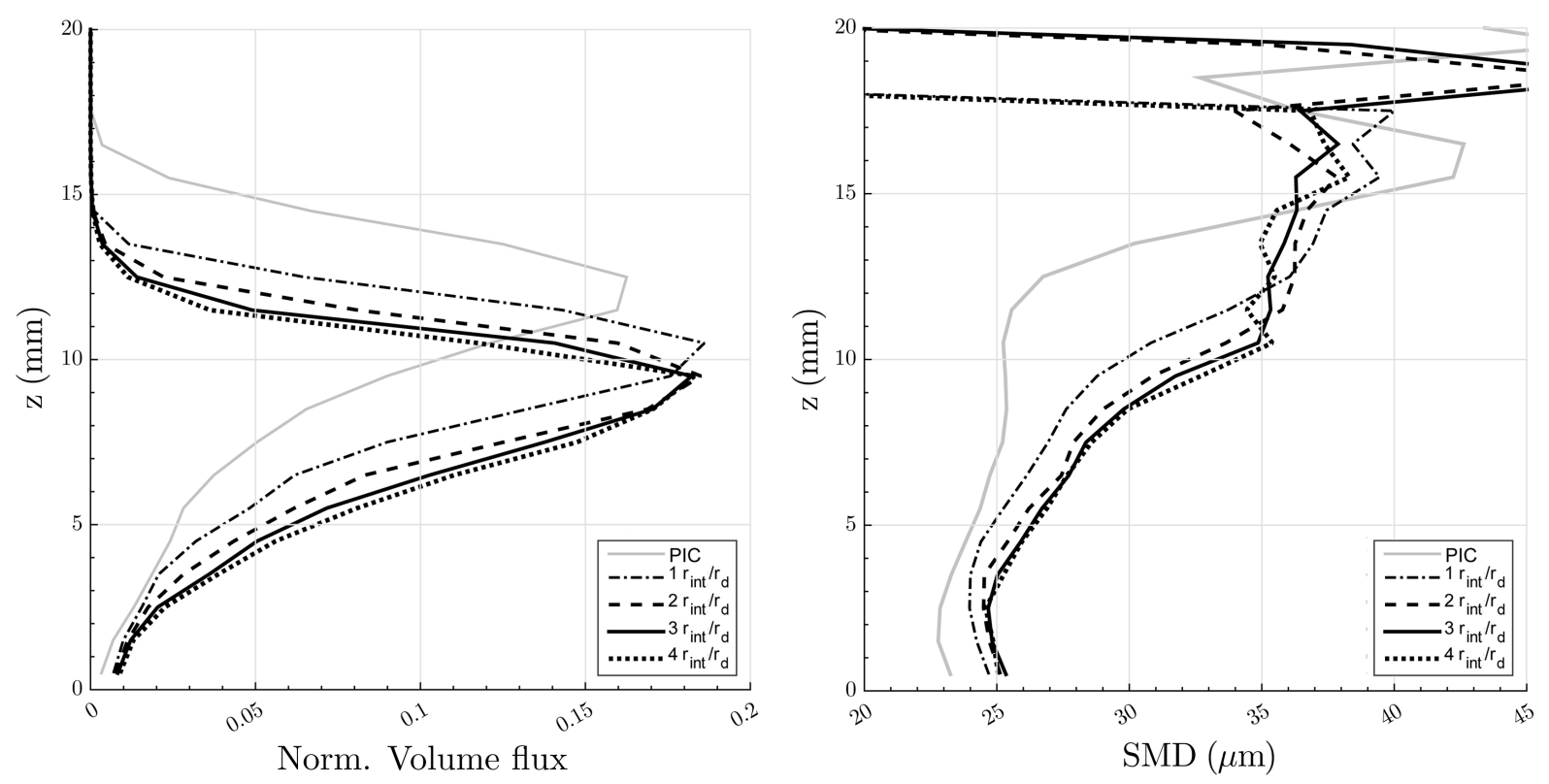

Figure 2.5: Effect of the source term distribution distance on the flux and SMD profiles. 
Following this, simulations with distribution distances from 1 to 4 times the droplet radius are carried out and the results are displayed in Figure 2.5. Similar results for both the integrated flux and the SMD are obtained for the highest interaction distances, while higher penetrations and smaller particle diameters are observed for lower distances. The PIC model is also evaluated as it is a particular case of the GPIC model if the distribution distance is small enough that the source terms are transferred only to the cell containing the parcel. In the latter case, due to the concentrated momentum transfer at that cell, the particles interpolate a significantly lower velocity than the one upstream of the jet and consequently the penetration increases. Additionally, no source terms are transferred to the cells located between the parcels that represent the jet, so that non-physical patterns of low and high velocities downstream of the jet are obtained.

Since the amount of ghost parcels increases with the interaction distance, a nondimensional interaction distance of 3 is selected for the subsequent simulations as a compromise between accuracy and computational expense.

\subsubsection{Mesh dependency of the PIC and GPIC models}

The impact of the spray resolution, in terms of the amount of parcels used, on the flux and SMD distributions at the sampling plane is evaluated before the mesh sensitivity study. To do so, three values of the $h_{i n j} / d_{i n j}$ ratio are employed. Since a value of 1 was used in the coupling model calibration, cases with double and half of that resolution are simulated. The results, plotted in Figure 2.6, indicate that the profiles are not significantly affected by this parameter. As the flux profile is slightly different for the lowest resolution case (i.e. $h_{i n j} / d_{i n j}=2$ ), a unity value is selected for the next simulations.
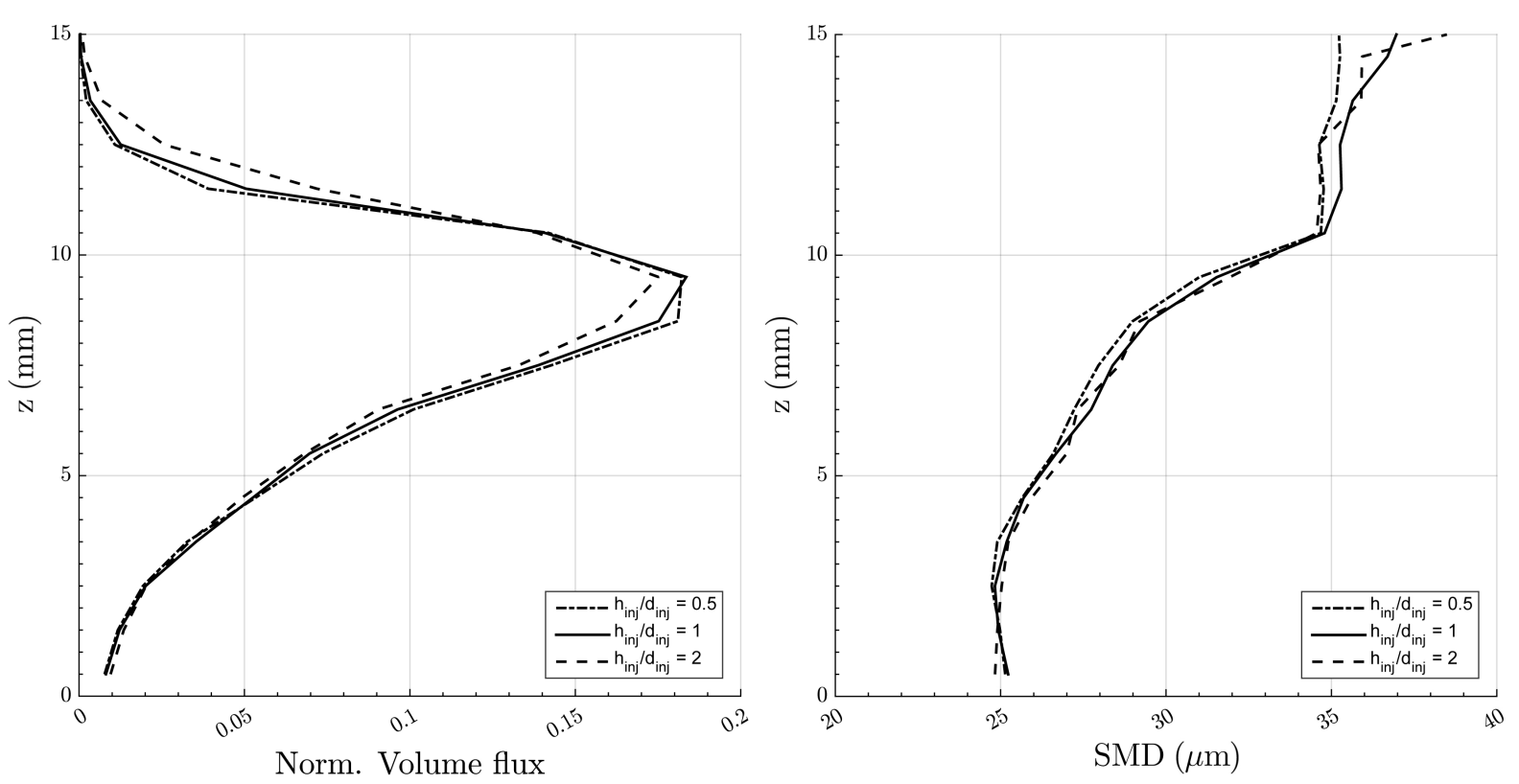

Figure 2.6: Influence of the injection rate on the integrated flux and SMD profiles. 

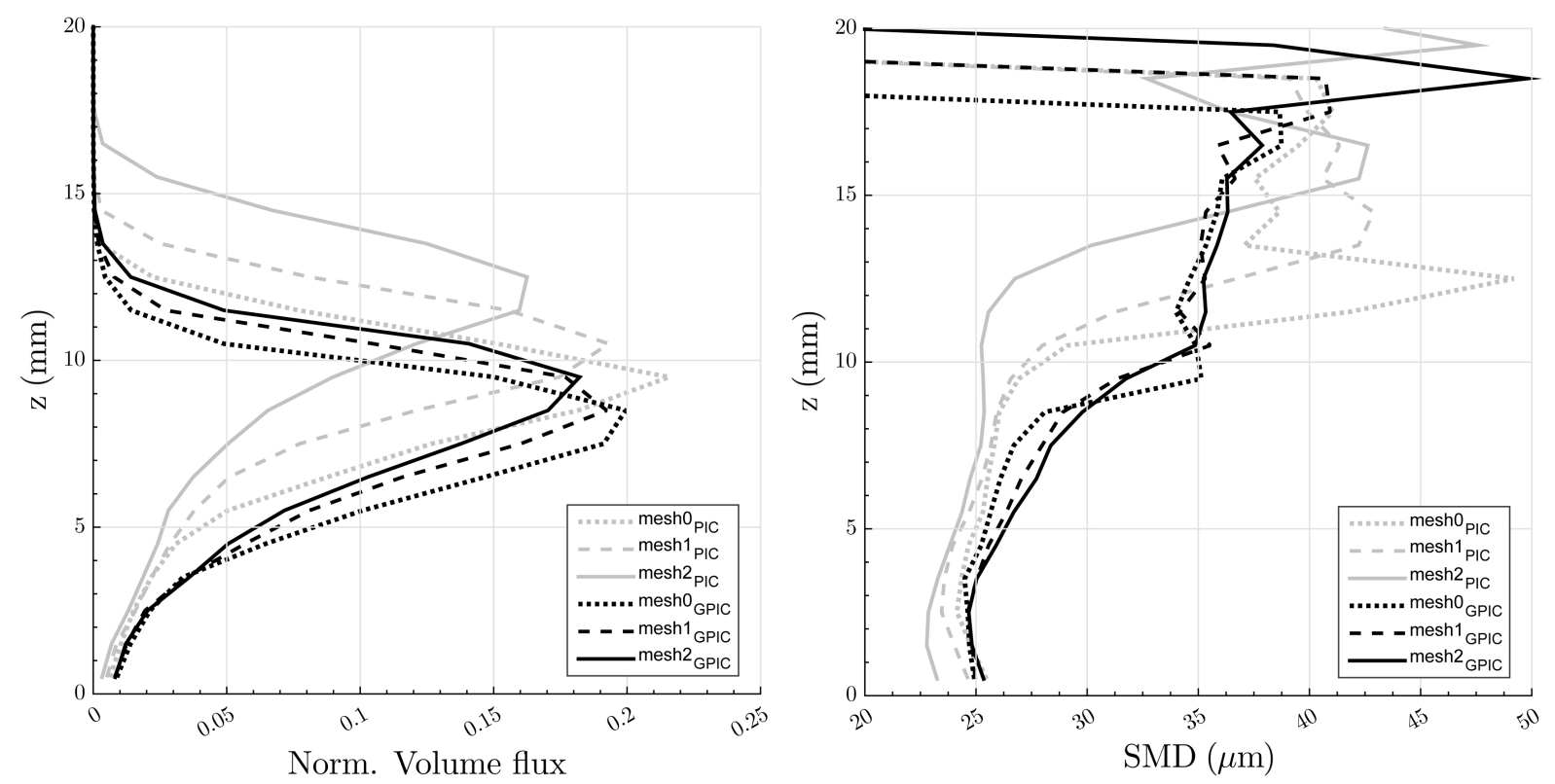

Figure 2.7: Influence of mesh refinements around the injection point on the integrated flux and SMD profiles for the GPIC and the PIC coupling models.

As aforementioned, the two coarser meshes are generated from the baseline mesh (mesh2) by removing all the refinement regions (mesh0) or only the finest one (mesh1). Simulation results at the sampling plane using both the PIC and the GPIC coupling models are plotted in Figure 2.7. In general, changes are more significant for the PIC model. In both cases the penetration increases as the mesh is refined, while the maximum flux value decreases. The SMD, however, is reduced with the PIC model and it increases slightly with the GPIC model.

Additionally, the trajectory of the jet and the primary droplets for the six cases are plotted together in Figure 2.8. In the PIC model, the higher source term concentration in the cell that contains the particle, due to the lower volume, leads to significantly larger trajectories for the finer meshes. With the source term distribution, the parcel is less affected by the volume of the cell where it is located and a similar trajectory is obtained for the three meshes considered. Thus, the advantage of the GPIC model is that, despite its higher computational cost, it has a low mesh dependency.

So far, only momentum coupling at low gas phase temperatures has been discussed. To complete the mesh sensitivity analysis, heating-up sprays are considered using the Ranz-Marshall heat transfer model [20]. In this way, the energy coupling is also evaluated with both the PIC and the GPIC model. The inlet gas temperature is set to $700 \mathrm{~K}$ and the mass flow is adjusted to keep a momentum ratio of 6 . Three coupling configurations are analysed:

- PIC model for both momentum and enthalpy,

- GPIC model for both momentum and enthalpy, 


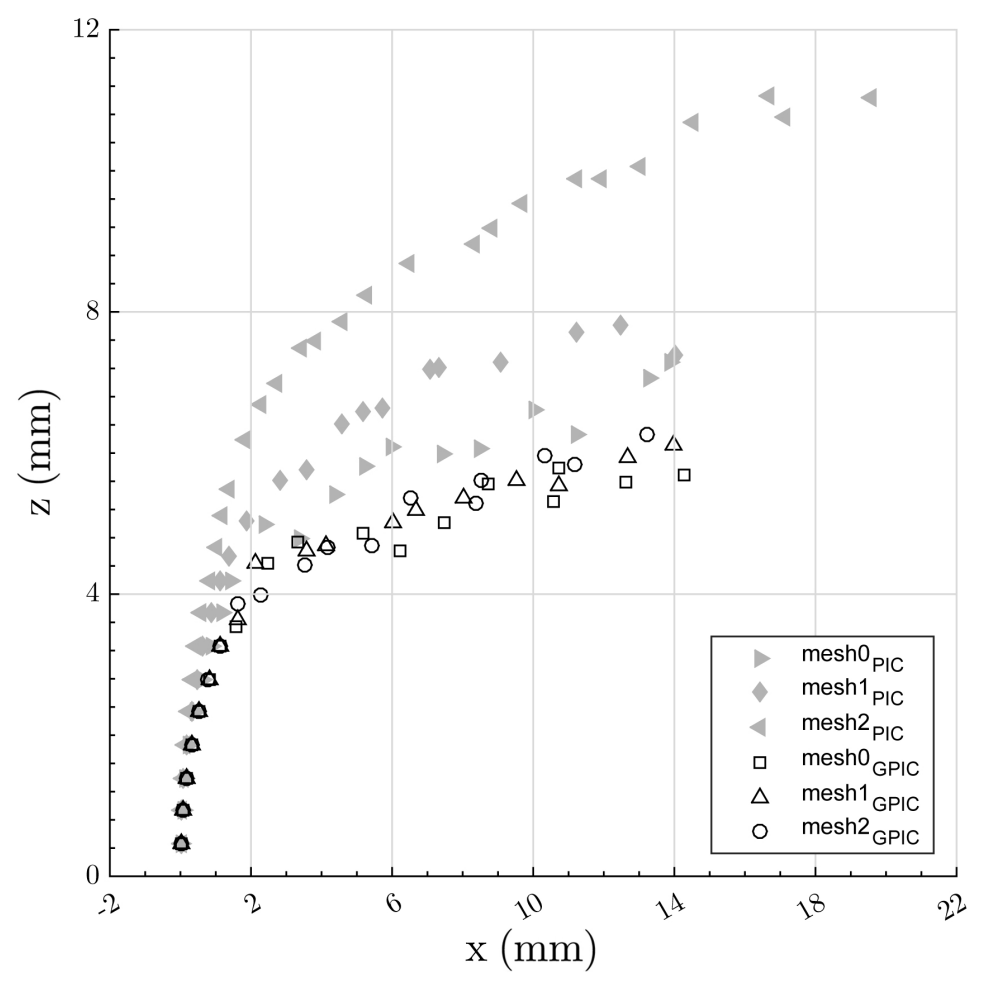

Figure 2.8: Influence of mesh refinements around the injection point on the jet trajectory for the GPIC and the PIC coupling models.

- GPIC model for momentum and PIC model for enthalpy.

The first two cases are compared on the left graph of Figure 2.9 by plotting the mean velocity profile at a vertical line located $80 \mathrm{~mm}$ downstream of the injection point. As in Figure 2.7, a higher mesh dependency is observed for the PIC model, since the influence height of the spray changes significantly, while there is little variation with the GPIC model.

Similarly, the last two configurations are compared on the right side of the figure by extracting the temperature profiles. The differences between the PIC and the GPIC models are smaller for the enthalpy coupling than for the momentum coupling. This is due to the large mass of the jet and primary droplets, so that they have a high inertia and a low surface-to-volume ratio. Both models result in a similar profile with the finest mesh and a lower minimum temperature is obtained as the mesh is coarsened, being this change larger for the PIC model.

In the case that evaporation is activated, the species' source term can also be distributed with the GPIC model. It is expected that the differences between the PIC and GPIC model for the species' profiles are smaller as there is no significant fuel evaporation from the jet and primary droplets due to their low temperature and surface area. A mesh sensitivity study of a n-heptane droplet evaporation time is investigated in Appendix B using both coupling models. For consistency, the GPIC model is applied to the momentum, energy and species coupling of the jet and primary parcels in the subsequent simulations. 

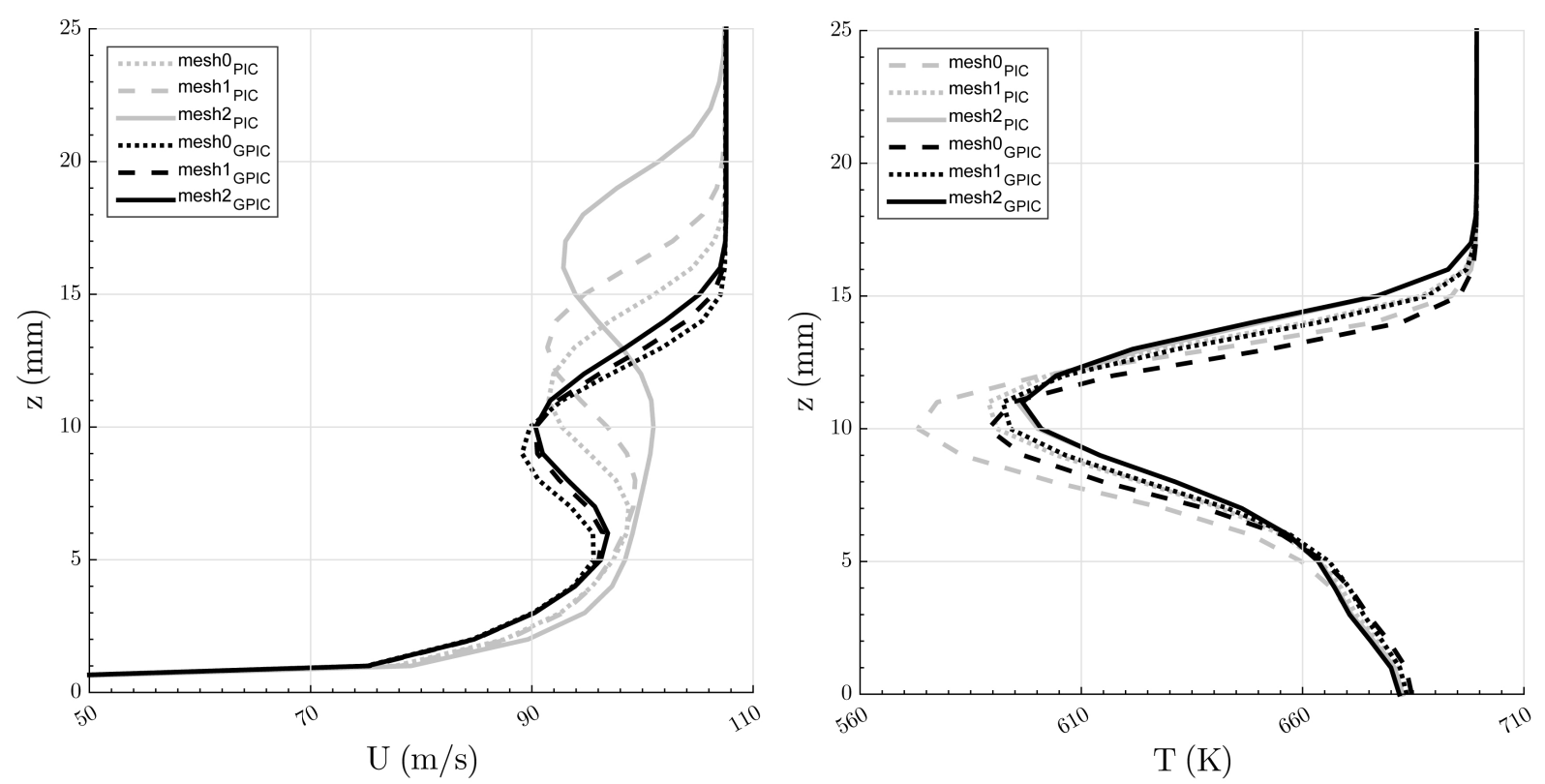

Figure 2.9: Influence of mesh refinements around the injection point on the gas phase velocity profile for the GPIC and the PIC coupling models.

\subsubsection{Validation}

Following the calibration and sensitivity study, the models are validated using the same experimental data as Rachner et al. [14]. Six cases with different liquid mass flow, inlet air velocity and pressure level are considered. The relevant boundary conditions for each case, together with the corresponding momentum ratio, are included in Table 2.1.

Table 2.1: Relevant boundary conditions used for the simulation of the validation cases.

\begin{tabular}{c||cccc} 
& $U_{\text {in }}(\mathrm{m} / \mathrm{s})$ & $P(\mathrm{bar})$ & $\dot{m}_{l}(\mathrm{~g} / \mathrm{s})$ & $q$ \\
\hline \hline q6 & 100 & 5.8 & 2.982 & 6 \\
q18 & 100 & 5.8 & 5.166 & 18 \\
q2 & 100 & 5.8 & 1.712 & 2 \\
u75q2 & 75 & 5.8 & 1.283 & 2 \\
u75q6 & 75 & 5.8 & 2.224 & 6 \\
p9 & 100 & 9 & 3.631 & 6
\end{tabular}

The liquid volume flux distributions from both simulation and experimental data are plotted in Figure 2.10 for the $q 6$ case. Numerical results show an over-prediction of the maximum flux position and lower spray dispersion. The latter may be caused by the partial resolution of the turbulence, due to the spatial filtering, or by the uncertainty in the turbulent scales imposed at the inlet boundary and it leads to a higher liquid concentration at the centre.

For a quantitative comparison, depth-integrated SMD and normalized volume flux 


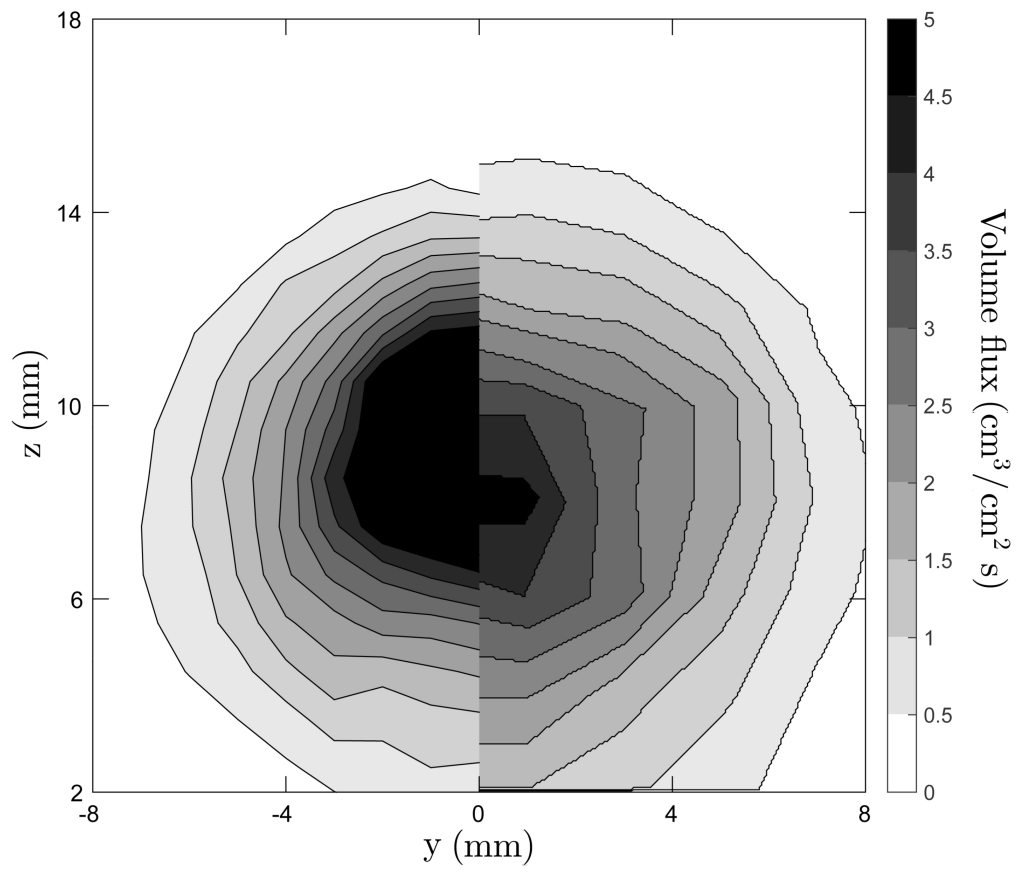

Figure 2.10: Comparison of the time-averaged volume flux distribution between experimental data from Rachner et al. [14] (right) and simulation results (left) for the $q 6$ case.

profiles over the channel height are plotted in Figure 2.11. As in the contour, the maximum flux value as well as its location is over-predicted by the model. Since the integral of the volume flux profile over the height is the same in both the simulation and the experiment, the flux at the bottom side is under-predicted. The SMD is in a better agreement as the largest discrepancy with the data is about $3 \mu \mathrm{m}$ at $z=5 \mathrm{~mm}$.

Norm. Volume Flux

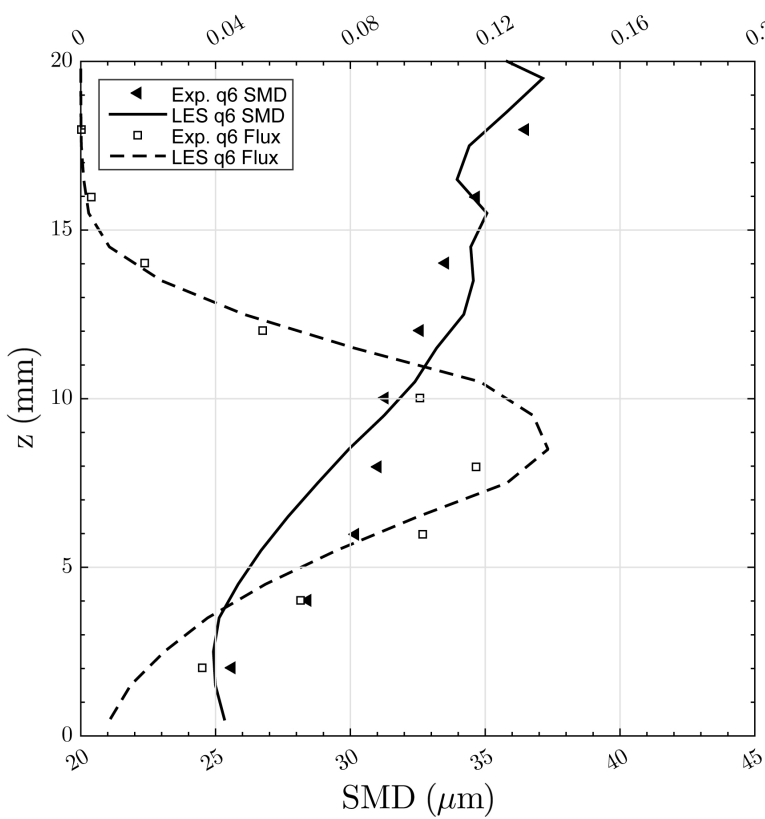

Norm. Volume Flux

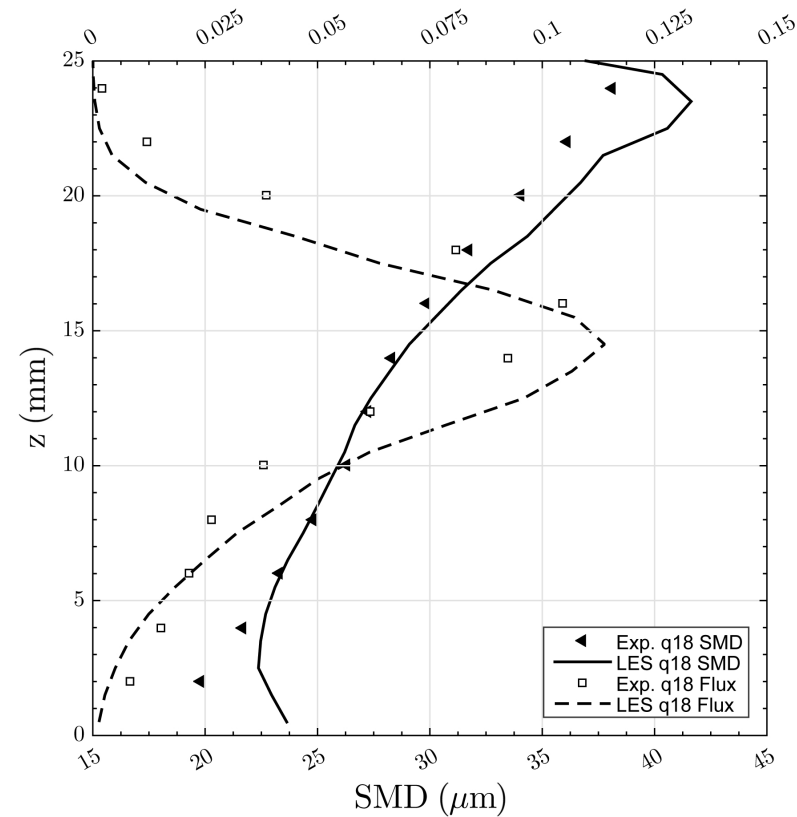

Figure 2.11: Comparison of the depth-integrated volume flux and SMD profiles with experimental data from Rachner et al. [14] for the $q 6$ (left) and $q 18$ (right) cases. 
Results for the higher momentum ratio, which are also included on the right side of the figure, show that the penetration depth increases in reference to the $q 6$ case while the SMD is reduced, specially at low heights. The model is able to reproduce this trend, with a good agreement in the SMD profile and a slight under-prediction of the maximum flux location.
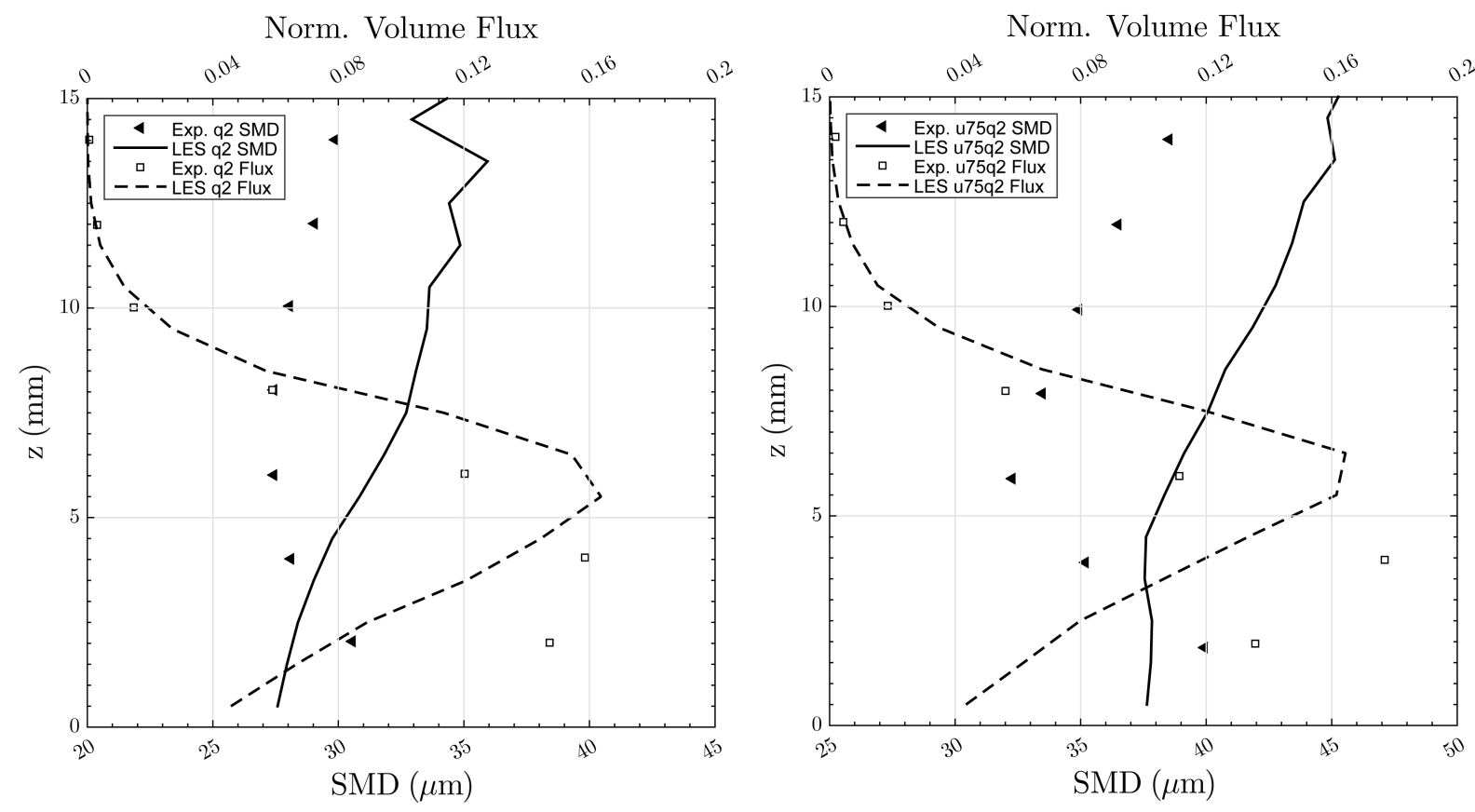

Figure 2.12: Comparison of the depth-integrated volume flux and SMD profiles with experimental data from Rachner et al. [14] for the q2 (left) and u $^{2} 5 q^{2}$ (right) cases.
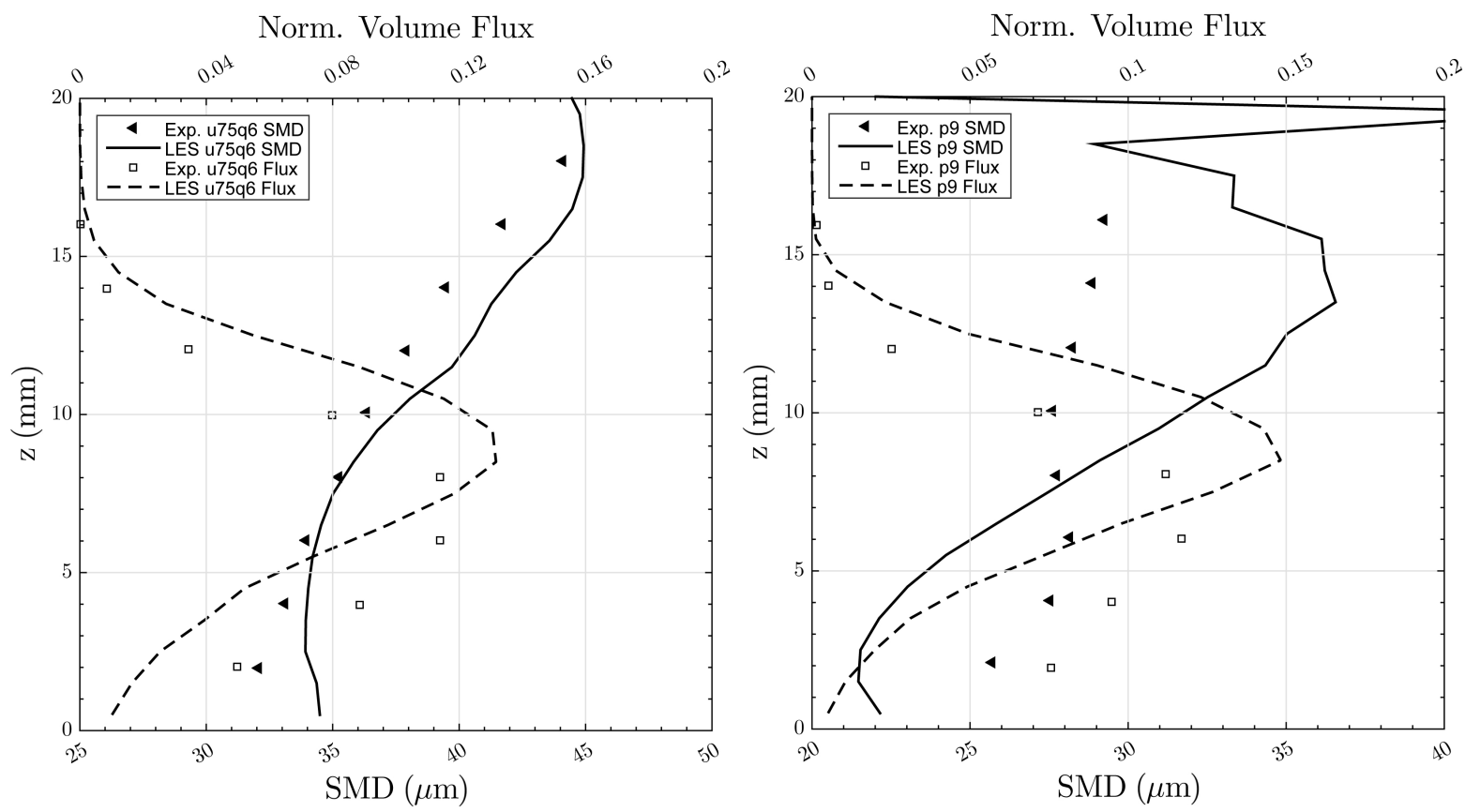

Figure 2.13: Comparison of the depth-integrated volume flux and SMD profiles with experimental data from Rachner et al. [14] for the $475 q 6$ (left) and $p 9$ (right) cases. 
The comparison of the results for the low momentum ratio cases are plotted in Figure 2.12. Experimental data shows about $4 \mathrm{~mm}$ difference in the spray penetration between the $q 6$ and $q 2$ cases. Regarding the SMD, a larger value than in the $q 6$ case is obtained near the wall, but it decreases as the maximum flux position is reached and increases at larger heights. Thus, the overall SMD is lower. A higher position of the maximum flux is obtained with the model, as in the $q 6$ case, but the parabolic shape of the SMD is not captured. The latter leads to an over-prediction of the SMD, specially at high penetration depths, with a maximum deviation of $10 \mu \mathrm{m}$.

The reduction of the upstream velocity from 100 to $75 \mathrm{~m} / \mathrm{s}$ implies a decrease in the fuel mass flow to maintain the momentum ratio. Taking the $q^{2}$ case as a reference, the location of the maximum flux in the $475 q 2$ case does not change significantly. However, the spray is more concentrated at that position as a higher flux value is obtained. The SMD profile is between 5 and $10 \mu \mathrm{m}$ higher along the complete channel height, and it also presents a parabolic shape. It can be observed that the change in the flux and SMD values is well captured by the numerical model, but the parabolic shape of the SMD profile is missed in these cases too.
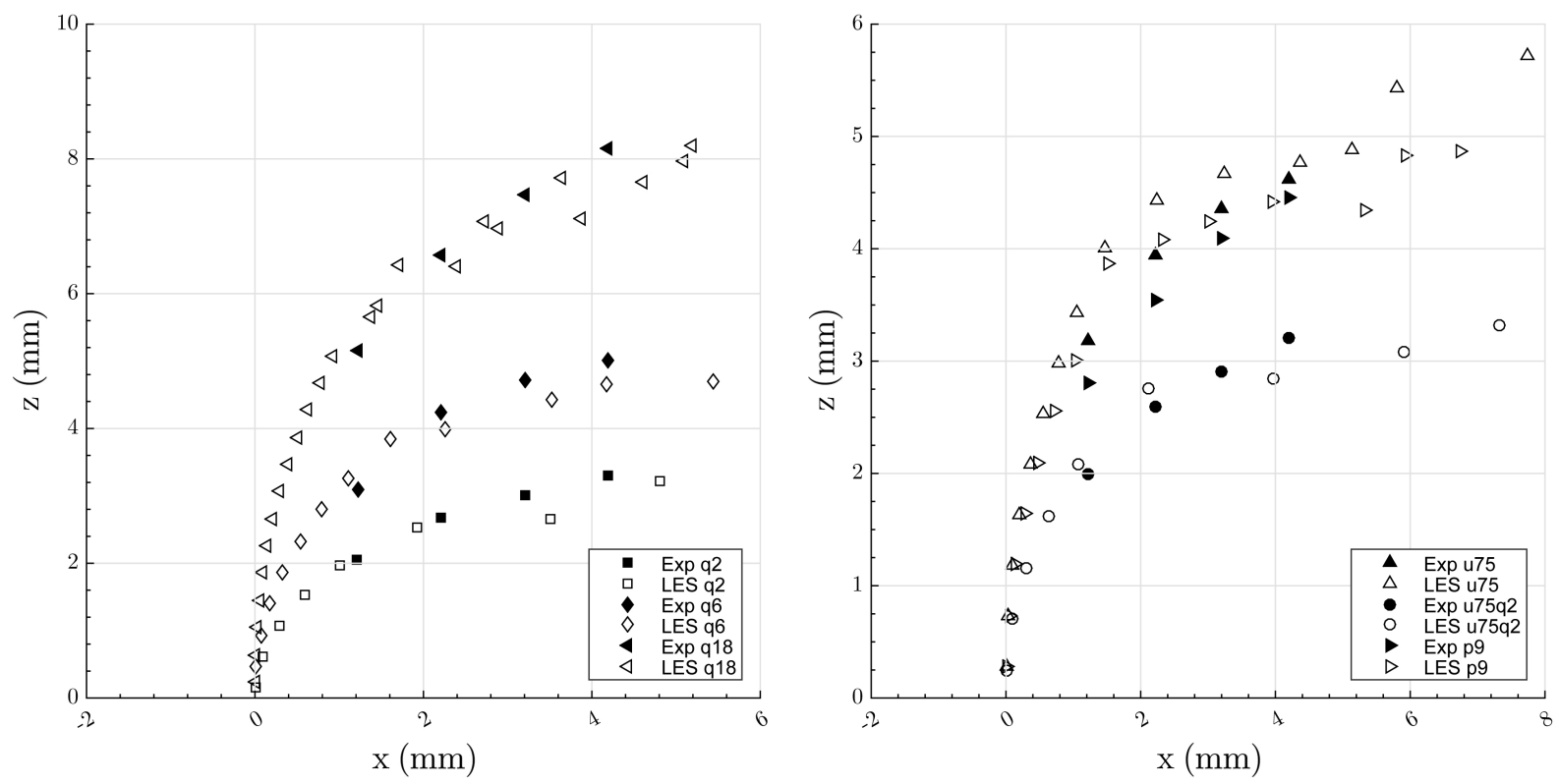

Figure 2.14: Comparison of the trajectory with experimental data from Rachner et al. [14].

In the same way, results for the $u 75 q 6$ and $p 9$ are displayed in Figure 2.13. Both cases show similar flux profiles as the $q 6$ case: the maximum flux value and height is over-predicted by the model. Regarding the SMD, as the fuel flow is raised from the 475q2 case up to $q=6$, it turns into a monotonically increasing profile and the model gives a better prediction. Furthermore, as for the low momentum ratio cases (i.e. $q=$ 2 ), the reduction in the upstream velocity while maintaining the momentum ratio results in an SMD increase of about 5 to $10 \mu \mathrm{m}$. The increase in the pressure level leads to a flatter rise of the SMD with the channel height, being the initial value the same as in the 
q6 case. Discrepancies with the numerical results are more evident in this case, with a maximum difference of about $10 \mu \mathrm{m}$, as droplet sizes are under-predicted near the wall and over-predicted at larger heights.

Apart from the flux and SMD distributions at a downstream plane, jet trajectories were also measured for each case. The comparison with the the numerical simulations is made in Figure 2.14. As the computation of a time-averaged jet trajectory is not possible in the OpenFOAM version used, the lagrangian parcels are retrieved from the last written time so that instantaneous trajectories are plotted. In general, the spray penetration in the first two millimeters is slightly over-predicted, while a lower value than in the experiments is obtained further downstream. Despite this, the trends observed between the cases are well predicted by the model.

\section{References}

[1] F. H. Harlow. A Machine Calculation Method for Hydrodynamic Problems. Tech. rep. Los Alamos Scientific Laboratory, 1955.

[2] M. W. Evans and F. H. Harlow. The Particle-in-Cell Method for Hydrodynamic Calculations. Tech. rep. Los Alamos Scientific Laboratory, 1957.

[3] A. Vallier. Tutorial icoLagrangianFoam/solidParticle. Lunds Tekniska Högskola.

[4] M. J. Andrews and P. G. O'Rourke. "The Multiphase Particle-in-Cell Method For Dense Particle Flows". In: International Journal of Multiphase Flow 22 (1996), pp. 379-402.

[5] Krista Stalsberg-Zarling. "Liquid-Gas Phase Coupling by means of Lagrange Polynomials for the CFD Simulation of High-Velocity Dense Sprays". PhD thesis. Michigan Technological University, 2007.

[6] S. K. Aggarwal et al. "Numerical optimization studies of axisymmetric unsteady spray". In: Journal of Computational Physics 50 (1983), pp. 101-115.

[7] N. Nordin. "Complex Chemistry Modeling of Diesel Spray Combustion". PhD thesis. Chalmers University of Technology, 2000.

[8] P. Beard et al. "Extension of Lagrangian-Eulerian spray modeling: application to high pressure evaporating diesel sprays". In: SAE Technical Paper 2000-01-1893. 2000 .

[9] P. J. O'Rourke and A. A. Amsden. "The TAB method for numerical calculation of spray droplet breakup". In: SAE International Fuels and Lubricants Meeting and Exposition. 1987. 
[10] M. Pilch and C. A. Erdman. "Use of breakup time data and velocity history data to predict the maximum size of stable fragments for acceleration induced breakup of a liquid drop". In: International Journal of Multiphase Flow 13 (1987), pp. 741-757.

[11] R. D. Reitz. "Modelling atomization processes in high pressure vaporizing sprays". In: Atomization and Spray Technology 3 (1987), pp. 309-337.

[12] J. C. Beale and R. D. Reitz. "Modeling spray atomization with the Kevin-Helmholtz/RayleighTaylor hybrid model". In: Atomization and Sprays 9 (1999), pp. 623-650.

[13] R. Schmehl, G. Maier and S. Witting. "CFD analysis of fuel atomization, secondary droplet breakup and spray dispersion in the premix duct of a LPP combustor". In: Eighth International Conference on Liquid Atomization and Spray Systems. 2000.

[14] M. Rachner et al. "Modelling of the atomization of a plain liquid fuel jet in crossflow at gas turbine conditions". In: Aerospace Science and Technology 6 (2002), pp. 495506.

[15] D. R. Guildenbecher, C. López-Rivera and P. E Sojka. "Droplet Deformation and Breakup". In: ed. by Nasser Ashgriz. Springer US, 2011, pp. 145-156.

[16] R. A. Gingold and J. J. Monaghan. "Smoothed Particle Hydrodynamics". In: Monthly Notices of the Royal Astronomical Society 181 (1977), pp. 375-389.

[17] D. Violeau. Fluid mechanics and the SPH method: Theory and applications. Oxford University Press, 2012.

[18] B. Werdelmann et al. "Open Boundary Conditions Using the Mirror Ghost Particle Approach in OpenFOAM SPH”. In: 9th SPHERIC International Workshop. 2014.

[19] M. Klein, A. Sadiki and J. Janicka. "A digital filter based generation of inflow data for spatially developing direct numerical or large eddy simulations". In: Journal of Computational Physics 186 (2003), pp. 652-665.

[20] W. E. Ranz and W. R. Marshall. "Evaporation from drops". In: Chemical Engineering Progress 48 (1952), pp. 141-146. 


\section{Chapter 3}

\section{Evaporation model}

\subsection{Introduction and state of the art}

In the simulation of liquid sprays within the Euler-Lagrangian formulation, the injection and atomization models result in a statistical distribution of particle sizes downstream of the injection point. As the fuel volatility increases, either because of the use of a lighter fuel component or a larger droplet temperature, the use of an evaporation model becomes necessary due to the phase change of the liquid to gas. The objective of this model is to predict the amount of evaporated mass from each liquid species transferred to the gas phase and the heat absorbed by the liquid phase. This is done based on the properties of the liquid and gas phases as well as on the flow structure inside and around the droplet.

In evaporation modelling, gas phase quasi-steadiness and spherical symmetry is usually assumed [1,2]. The classical evaporation model was proposed by Godsave [3] and Spalding [4], considering a steady state evaporation by assuming a constant droplet temperature. This model is also know as the $d^{2}$-law.

Unsteady effects were investigated by Law [5] and Hubbard et al. [6], assuming a spatially uniform time-varying droplet temperature (infinite liquid conductivity). A nonuniform temperature inside the droplet was modelled by Maqua et al. [7], assuming a polynomial profile. The 1-D model by Abramzon and Sirignano [8] uses an effective liquid conductivity in the energy equation to account for internal recirculation, which is calculated assuming a Hill vortex flow inside the droplet. Influence of natural and force convection are accounted by using correlations for the Sherwood and Nusselt numbers $[9$, 10], or by applying corrective factors to the model to account for the formation of a film layer around the droplet [11]. Non-unity Lewis number of the gas phase, Stephan flow and spray interaction effects can also be accounted for $[8,2]$.

Additionally, ambient pressure plays a significant role in the droplet evaporation process, especially when it is near or above the liquid critical pressure [12], since it affects the liquid and gas phase properties as well as the vapour-liquid equilibrium. Several 
authors $[13,12,14,15,16,17]$ employ the Peng-Robinson equation of state to calculate the fugacity of the liquid and gas phases to solve the vapour-liquid equilibrium. A model for evaporation of oxygen droplets at sub-, trans- and supercritical conditions was developed by Delplanque and Sirignano [18] and applied to the evaporation of parallel droplet streams in [19]. A numerical investigation on the evaporation oxygen droplets at several high temperatures and pressures, from subcritical to supercritical conditions, was performed by Haldenwang et al. [20]. Combustion of fuel droplets at supercritical conditions was analysed by Shen [21] based on the conservation equations for each phase.

A model for multicomponent droplets was first developed by Landis and Mills and applied to binary droplets [22]. Many other multicomponent models were developed [23, $24,12,14,25,26,27]$ to account for the preferential evaporation of the more volatile components within the droplet. These models are also known as discrete multicomponent models, since a transport equation must be solved for each liquid species [28]. Continuous multicomponent models - as proposed in $[29,30]$ - are based on the description of the fuel composition using a PDF for a key fuel property, being the molecular weight the most utilized. Liquid properties are calculated from correlations that depend on the key property only, if not assumed constant, and transport equations are solved for the parameters that describe the PDF [28].

The use of emulsified fuels can enhance the atomization process, by means of the droplet micro-explosion phenomena [31], and reduce the soot and NOx emissions [32, 33, 34]. Regarding the evaporation of emulsion droplets, Law [35] proposed the frozen and the distillation limits. In the frozen limit the water and fuel mass fractions do not vary with time. In the distillation limit, the water and fuel are vaporized independently assuming that water is always available at the droplet surface. Leite and Lage [25] proposed an interface model so that the mole fraction of water at the droplet surface is limited by the amount of water available at the interface. Le Clercq et al. [26] proposed the segregated distillation mode, which limits the water mass fraction at the liquid-gas interface to the water mass fraction in the droplet core. This segregated mode was combined with the shell model. It assumes the formation of an oil shell layer at the droplet surface, due to water depletion, which prevents further evaporation of water. The latter is also supported by $\mathrm{Fu}$ et al. [36], who states that an oil layer is created for both $\mathrm{W} / \mathrm{O}$ and $\mathrm{O} / \mathrm{W}$ emulsion types. More recently, Morozumi et al. [37] proposed a 1-D model that accounts for the transfer of heat from the fuel to the dispersed water droplets by considering a additional temperature variable for the water. For the prediction of the micro-explosion phenomena, the homogeneous nucleation approach [31] is commonly used to determine the superheat temperature at which this event occurs. 


\subsection{Model}

The evaporation model employed here is the zero-dimensional multicomponent model taken from Le Clercq et al. [26]. In his work, the model was validated for pure heptane droplets and extended to emulsified droplets. The model consists of the following differential equations that describe the evolution of the droplet mass, temperature and composition with time:

$$
\begin{gathered}
\frac{d M_{d}}{d t}=-\pi d \rho_{s} \text { Sh } D_{s} \ln \left(1+B_{m}\right) \\
\frac{d T_{d}}{d t}=\frac{6 \alpha_{a}}{\rho_{l} C_{p, l} d^{2}} N u\left(T_{\infty}-T_{d}\right)+\frac{1}{M_{d} C_{p, l}} \sum\left(\frac{d M_{d, i}}{d t} L_{v, i}\right) \\
\frac{d Y_{i}}{d t}=\frac{1}{M_{d}} \frac{d M_{d}}{d t} \frac{Y_{i, s}-Y_{i}}{B_{m}}
\end{gathered}
$$

The Nusselt and Sherwood numbers are calculated from correlations according to Le Clercq et al. [26], which are based on the Ranz-Marshall [10] and Frössling [9] equations. This assumes that the heat and mass transfer take place inside a thin film that surrounds the droplet [38]. For natural convection conditions, where the $G r>10 R e$, Equation (3.4) is used. When force convection dominates, with $R e>10 \mathrm{Gr}$, Equation (3.5) is utilized. For intermediate conditions, both effects are important and Equation (3.6) is applied.

$$
\begin{gathered}
S h_{N C}=2+0.43\left(\begin{array}{ll}
G r & S c
\end{array}\right)^{0.25} \\
N u_{N C}=2+0.43\left(\begin{array}{ll}
G r & P r
\end{array}\right)^{0.25} \\
S h_{F C}=2+0.552 R e^{0.5} S c^{0.33} \\
N u_{F C}=2+0.552 R e^{0.5} \operatorname{Pr}^{0.33} \\
S h_{N C+F C}=\sqrt{S h_{F C}^{2}+S h_{F C}^{2}} \\
N u_{N C+F C}=\sqrt{N u_{F C}^{2}+N u_{F C}^{2}}
\end{gathered}
$$

For the pressure range of interest, up to 30 bar, it is assumed that the use of the latent heat (instead of the enthalpy of vaporization) in the energy equation is adequate. The parameter $B_{m}$ is the Spalding number for mass transfer, which is defined as:

$$
B_{m}=\frac{\sum\left(Y_{i, s}-Y_{i, \infty}\right)}{1-\sum Y_{i, s}}
$$

Both the liquid and the gas properties for each component can be calculated using appropriate correlations from Poling et al. [39] and Perry and Green [40]. These properties are then mass-averaged to obtain the overall droplet properties that are used in the equations. A reference temperature for the liquid-gas interface is calculated from the 
equation below $[6]$ :

$$
T_{s}=\frac{2}{3} T_{d}+\frac{T_{\infty}}{3}
$$

Using this temperature and the ambient pressure, the mixture fractions at the interface are calculated from the vapour pressure of each component. At low pressures, the ratio between each component's vapour pressure and the mixture pressure equals the molar fraction of the corresponding component at the droplet surface. This is known as Raoult's law. At high pressures, from 15 bar on, real gas effects become important and the surface concentrations are determined assuming that the liquid fugacity equals the gas fugacity for each component i:

$$
f_{i, g}=\chi_{i, g} P \Phi_{i, g}=\chi_{i, l} \gamma P_{s a t} \Phi_{i, s a t}=f_{i, l}
$$

This model can be extended to emulsified droplets by modelling how the water component in the droplet is vaporized. The amount of water used in heavy-duty gas turbines can reach up to $50 \%$ the mass flow of the injected liquid. For such water contents, at the pressure levels considered, the water is assumed to be immiscible with the oil. The model proposed in $[26,36]$ assumes a uniform distribution of the dispersed water droplets. It consists in the formation of an oil shell layer at the droplet surface, whose thickness is determined by the characteristic diameter of the dispersed phase, due to the depletion of water micro-droplets at that region. This fuel layer prevents further evaporation or boiling of water droplets since they are encapsulated within the droplet.

\subsubsection{Major model adjustments}

In the present work, the following model assumptions and extensions are implemented. Firstly, for the calculation of the species' concentration at the droplet surface, the fugacity coefficient is defined as:

$$
\ln \Phi=\int_{0}^{P} \frac{Z_{c}-1}{p} d p
$$

The compressibility factor is calculated using the Peng-Robinson equation of state, in which A and B depend on the species properties:

$$
Z_{c}^{3}+(B-1) Z_{c}^{2}+\left(A-2 B-3 B^{2}\right) Z_{c}+\left(B^{3}+B^{2}-A B\right)=0
$$

The fugacity of the saturated pressure can be neglected at low temperatures. High temperatures can only be achieved by having a high boiling point, which requires in turn elevated pressure. For the pressure range considered, this term is accounted. The activity coefficients depend mainly on the liquid composition and temperature, and they can only 
be determined experimentally. The term $\Phi_{i, g}$ depends on the temperature, pressure and composition of the mixture, which is also to be determined [39]. A coupled system of equations is obtained, which requires an iterative procedure along with an initial guess. As this process is computationally expensive, especially when many species describe the fuel composition, the Lewis fugacity approximation is made as a compromise. It assumes that the fugacity coefficient of a species in the gaseous mixture is the same as the fugacity of the gas species, which is a valid assumption for low pressures and moderate pressures when the components have similar molecular properties [41]. Despite the restriction of this assumption, a correction for pressure effects is performed (Raoult's law assumes that the fugacity coefficient is one) without a significant impact on the computational effort. The latter becomes important as the amount of parcels in the domain increases.

Once the mass fraction of each species is calculated at the droplet surface, the compressibility factor for this composition is computed using a mixing rule for the $A$ and $B$ coefficients in Equation (3.11) and the density at the interface is obtained:

$$
\rho_{s}=\frac{P W_{s}}{Z_{c} R T_{s}}
$$

where the molar mass at the interface is also calculated using a mass-averaged mixing rule. In the case of a multicomponent fuel droplet at high temperature ambient conditions, boiling has to be taken into account due to the different boiling points between light and heavy components. It is assumed that the boiling temperature is a specific property of each species, so that the vapour pressure calculated with the Antoine's equation does not exceed the mixture pressure. From Equation (3.2), the heat transferred from the gas phase to the droplet is employed in mass evaporation (calculated in Equation (3.1)) and temperature rise. From the time the droplet temperature reaches the boiling point of the most volatile component, however, it is assumed that all the heat received is used only in mass evaporation (i.e. constant droplet temperature is imposed) until that component is completely depleted. Therefore, during this period, Equation (3.2) is replaced by Equation (3.13) so that the additional evaporated mass of the boiling component $\mathrm{j}$ is calculated. After this equation is applied, the mass fraction of each component needs to be recalculated because of the additional mass transfer.

$$
\begin{aligned}
M_{d} C_{p} \frac{d T_{d}}{d t} & =0 \\
& =\pi d \alpha_{a} N u\left(T_{\infty}-T_{d}\right)+\frac{d M_{j, \text { boil }}}{d t} L_{v, j}+\sum\left(\frac{d M_{i}}{d t} L v_{i}\right)
\end{aligned}
$$

In order to improve the criteria for the oil shell formation in the evaporation of emulsion droplets, a radial diffusivity model for the dispersed phase is introduced. It consists in a transfer of water droplets from the core to the shell region. A similarity with the Fick's law is used, taking the characteristic length as the distance between the core centre and 
the mid shell radius:

$$
\frac{d M_{w, \text { core }}}{d t}=-\pi\left(d-2 d_{w}\right)^{2} \quad D_{w, \text { eff }} \frac{\chi_{w, \text { core }}-\chi_{w, \text { shell }}}{d-0.5 d_{w}}
$$

The effective diffusivity aims to account for the convection of droplets from the core to the shell due to internal flow recirculation. An analogy with the Brownian molecular motion [42], which also utilizes the Fick's law, can be used to account for the dependency of the diffusivity on the size of the water droplets, the fuel viscosity and the temperature:

$$
D_{w, e f f}=K_{e f f} \frac{k_{B} T}{6 \pi \mu_{f} d_{w}}
$$

Where $K_{\text {eff }}$ is a coefficient that can account for convective transport. In the present work, the diffusivity is assumed constant through the complete droplet lifetime. Once the fuel shell has been formed, this diffusivity model is not further applied and the temperature is allowed to rise over the water boiling point. Since a criteria for micro-explosion of the emulsion droplet is not included in the present work, once the fuel shell is vaporized the droplet structure is the same as at the initial state and the next shell is vaporized. As a rough estimation, it can be assumed that micro-explosion occurs when the first fuel shell layer is vaporized since it is followed by a rapid evaporation of the water that is present in the next layer.

\subsection{Results}

The evaporation model is evaluated by performing OpenFOAM simulations of an isolated droplet initialized at the center of a $0.2 \times 0.2 \times 0.2 \mathrm{~m}^{3}$ box with zero initial velocity. Each direction of this domain is discretized into 40 elements so that the volume of the cell containing the droplet is at least 10 times larger than the droplet volume. Otherwise, since the droplet takes the far-field values from the cell where it is located and transfers the source terms to that cell (2-way coupling), the results would be very sensitive to the mesh resolution. For all cases evaluated in this section, the droplet has been artificially fixed from its initialization since in the experiments the droplets are suspended by means of a quartz fiber, a glass capillary or a thermocouple.

\subsubsection{Single phase droplets}

Firstly, mono-component droplets are simulated and the results are compared against experimental data. In the experiments from Morin et al. [43] a quartz fiber was used to suspend the droplets and the heat transfer from the fiber was estimated to be about $6-11 \%$ of the heat received by the droplet from the gas phase. In both Figures 3.1 and 3.2 a $7 \%$ value was considered, as well as an initial droplet temperature of $300 \mathrm{~K}$. 


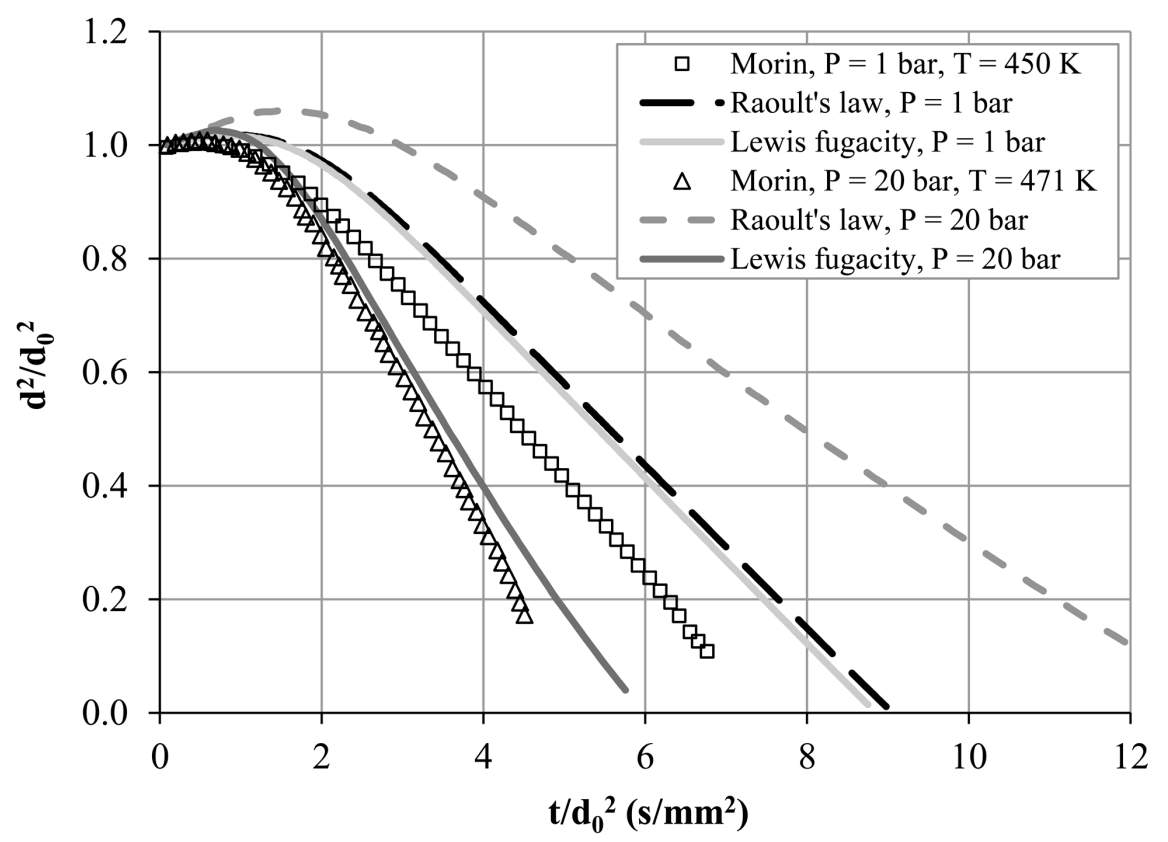

Figure 3.1: Model results of the droplet diameter evolution for n-decane droplets under natural convection compared against experimental data from Morin et al. [43].

Figure 3.1 shows the diameter evolution of a n-decane droplet at both atmospheric pressure and 20 bar under normal gravity from [43]. Model results using both the Raoult's law and the fugacity equilibrium with the Lewis assumption are also shown. At 1 bar, the fugacity approach gives very similar results to the Raoult's law. The over-prediction in the evaporation time with reference to the experimental data can be attributed to the uncertainty in the initial droplet temperature, as the heating period is too long, but the steady evaporation rate (i.e. slope of the curve) is similar to the one in the experiments. For 20 bar pressure the Raoult's law gives a much higher evaporation time. Such behaviour is expected since it is assumed that the vapour fugacity coefficient is one. The latter implies that the molar fraction at the droplet surface is inversely proportional to the mixture pressure (see Equation (3.9)), which reduces the evaporation rate. Therefore, if temperature is maintained, the Raoult's law always leads to longer heating periods and higher evaporation times when the pressure is increased. With the fugacity model, a shorter evaporation time than at atmospheric conditions is obtained due to the calculation of the fugacity coefficient using Equation (3.2.1). This trend is also observed in the experiments.

Focusing on the 20 bar condition, different gas phase temperatures are considered in Figure 3.2. As the air temperature increases, the discrepancies with the experimental data in the heating stage become significant. This is caused by the assumption of a spatially uniform droplet temperature, as large droplets are simulated (around $1.2 \mathrm{~mm}$ ), which causes a high droplet expansion. In the experiments, temperatures at the surface are higher and therefore there is significant evaporation at that region. For the typical 
droplet sizes in gas turbines after the atomization process, around $50 \mu \mathrm{m}$, this assumption is reasonable for the investigated temperatures. Additionally, the steady evaporation rate is over-predicted as temperatures increase. This result is likely to be caused by the calculation of the surface conditions, i.e. the Lewis assumption is less accurate. Despite this, the results obtained are better than if the Raoult's law is used and with a smaller computational effort than if the iterative procedure is performed to calculate the correct fugacity coefficients.

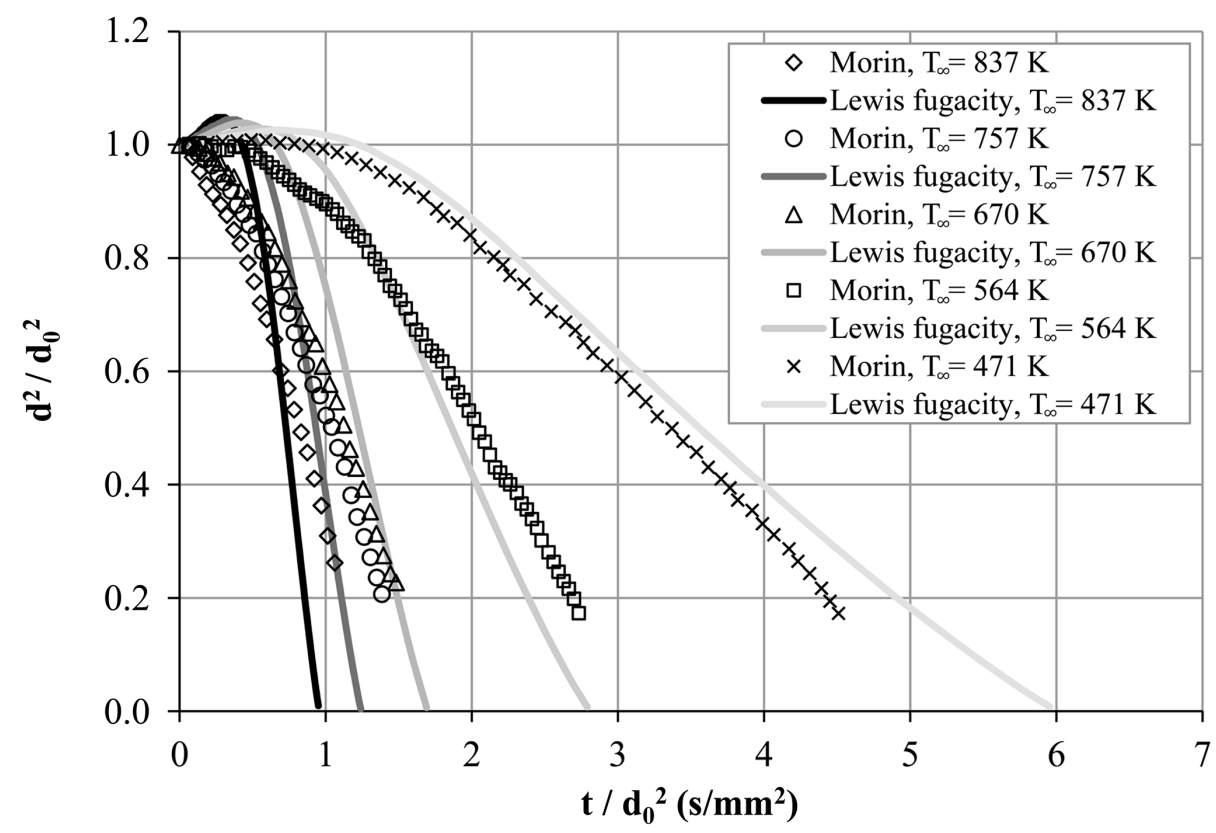

Figure 3.2: Model results of the droplet diameter evolution for n-decane droplets under natural convection at $P_{\infty}=20$ bar compared against experimental data from Morin et al. [43].

To evaluate the effect of natural and forced convection on multi-component droplets, experimental data from Daif et al. [38] is used. Due to the low ambient temperatures, the heat transferred through the capillary glass is neglected. The droplet is placed in the quiescent test section and it vaporizes under natural convection conditions. At certain time, a valve opens so that the droplet is under a higher temperature cross flow. In the simulations, this is done by imposing a fixed velocity value at one of the patches and zero gradient condition in the opposite boundary. Figure 3.3 shows that evaporation rate in the simulations is slightly over-predicted for natural conditions, but under forced convection a better agreement with the experimental data is obtained.

Additional cases for mono-component droplet evaporation under either natural or forced convection are included in Appendix B. In it, a mesh sensitivity study is also investigated using both the PIC and the GPIC coupling models. Further validation cases for the evaporation of n-heptane droplets under micro-gravity conditions can be found in Appendix C. 


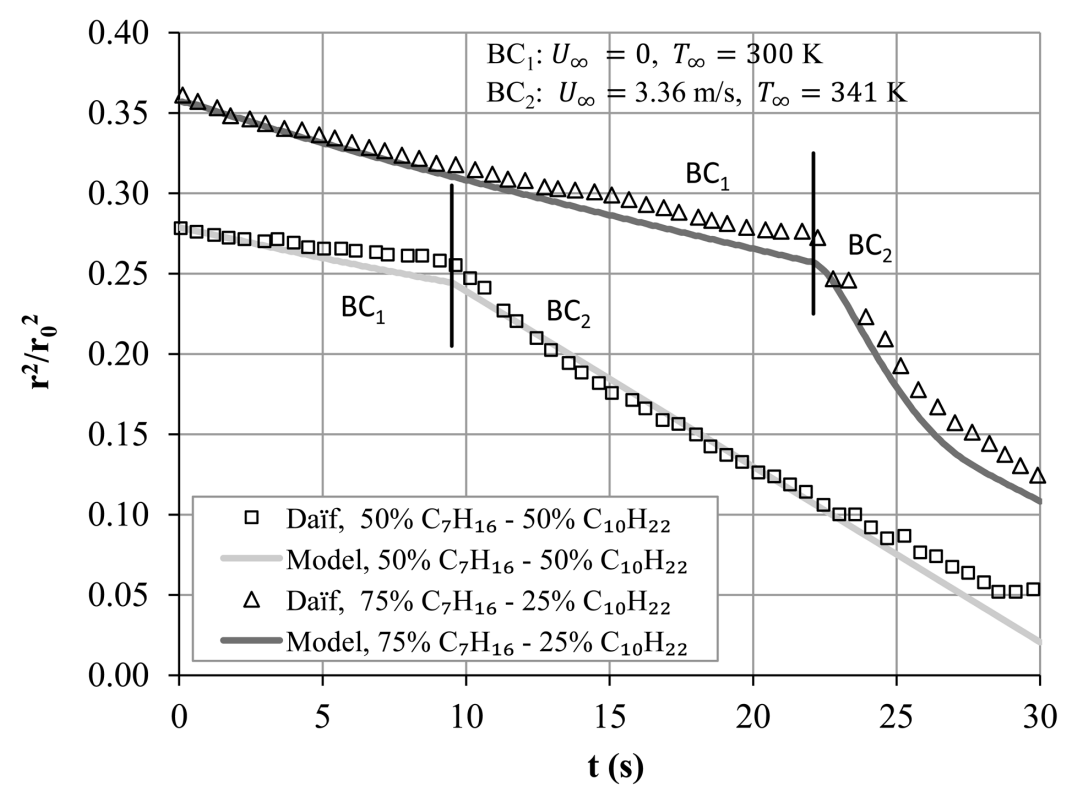

Figure 3.3: Model results of the droplet diameter evolution for n-decane/n-heptane droplets under both natural $\left(\mathrm{BC}_{1}\right)$ and forced $\left(\mathrm{BC}_{2}\right)$ convection compared against experimental data from Daif et al. [38].

\subsubsection{Emulsion droplets}

Regarding the evaporation of emulsion droplets, Figures 3.4 and 3.5 illustrate how the shell model, in combination with the water diffusivity model, works. For the same initial water content, with low diffusivity values a shell is created early in the heating period and the temperature increases beyond the water boiling temperature. When the oil shell is vaporized the water of the next layer is evaporated and the droplet is reheated afterwards, leading to a steady zig-zag curve.

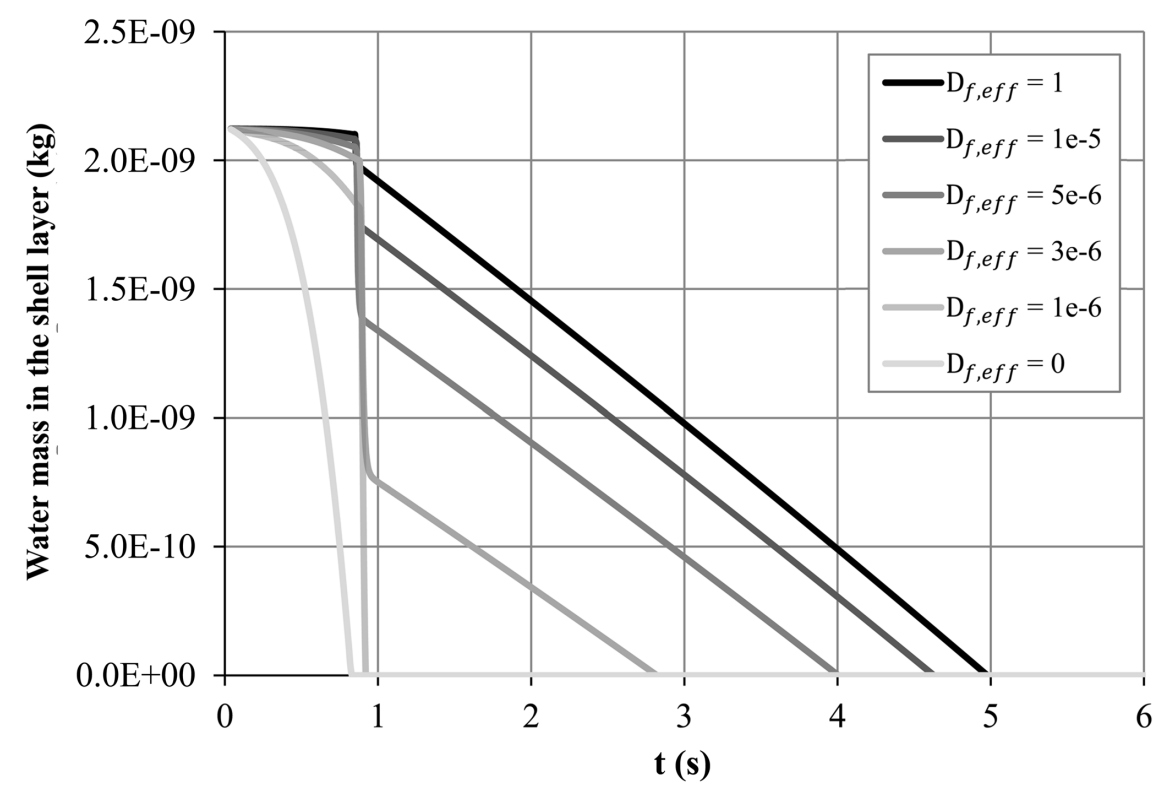

Figure 3.4: Influence of the diffusivity parameter on the droplet shell water content. 


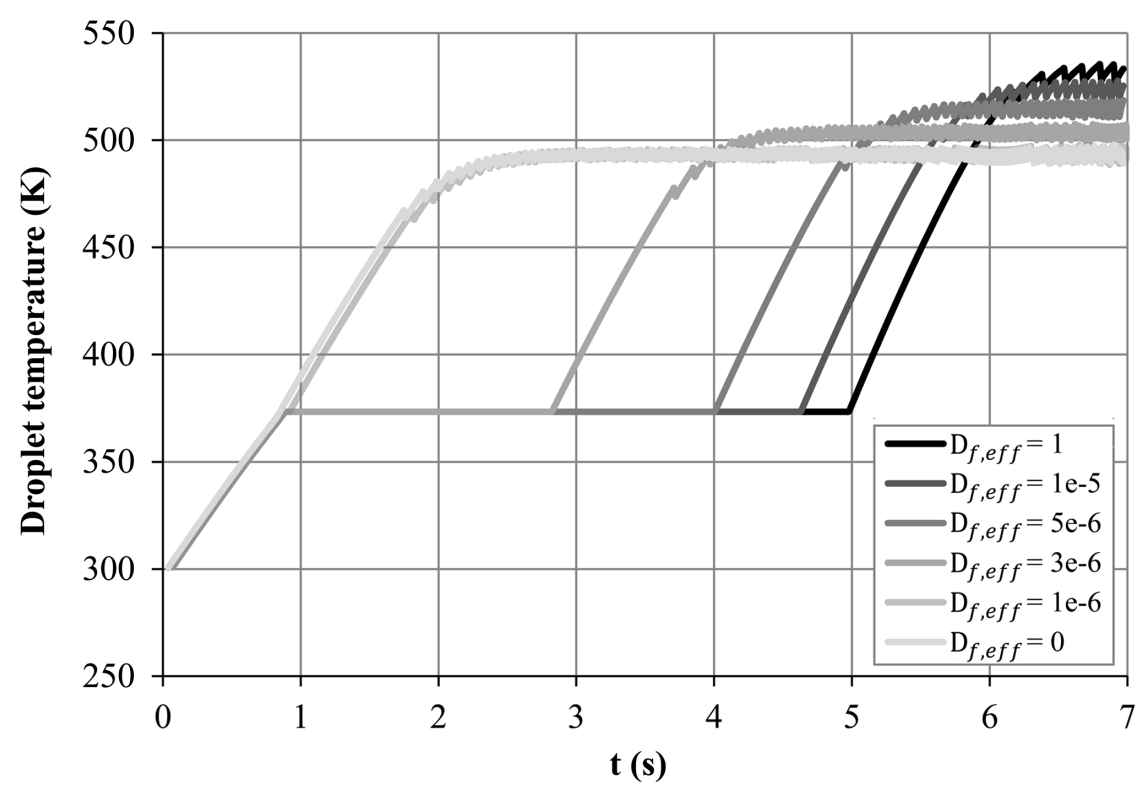

Figure 3.5: Evolution of the droplet temperature for several water diffusivity values.

As the diffusivity increases, more water is vaporized before the first oil shell is created and therefore the droplet temperature remains more time at the boiling temperature of water (i.e. the distillation limit is approached). A higher temperature is achieved when the shell is created, in comparison to a lower water diffusivity, because the amount of water evaporated from the shells is lower. Above a certain diffusivity value, very similar results are obtained since the maximum possible water is transferred at every time step to equilibrate the concentrations. This means that the diffusivity model does not allow the complete depletion of water, so that a shell is always formed and the distillation limit can never be achieved.

Experimental data for the evaporation of hexadecane-water droplets has been obtained from Morozumi et al. [37] and it is plotted in Figure 3.6 together with the model results. The size of the dispersed droplets was about $1 \mu \mathrm{m}$ and a surfactant with a HLB value of 4.3 was used to stabilize the emulsion. A droplet of about $1.5 \mathrm{~mm}$ was suspended in a thermocouple and introduced inside an electrical furnace at a temperature of $800^{\circ} \mathrm{C}$ and at atmospheric pressure. Since the temperature is measured at the droplet centre, an initial delay period can be observed and the results of the simulations are shifted accordingly.

As the heat transfer in the simulations was not enough to reproduce the temperature increase observed in the experiments, and since radiation effects from the furnace walls are expected to be important, a larger ambient temperature has been used in the simulations. This also accounts for any heat transferred from the thermocouple. The same temperature has been used for the different water contents and the comparison with experiments indicates that the effect of water in the initial heating period is well predicted. The effective diffusivity has also been adjusted in order to follow the second increase in 


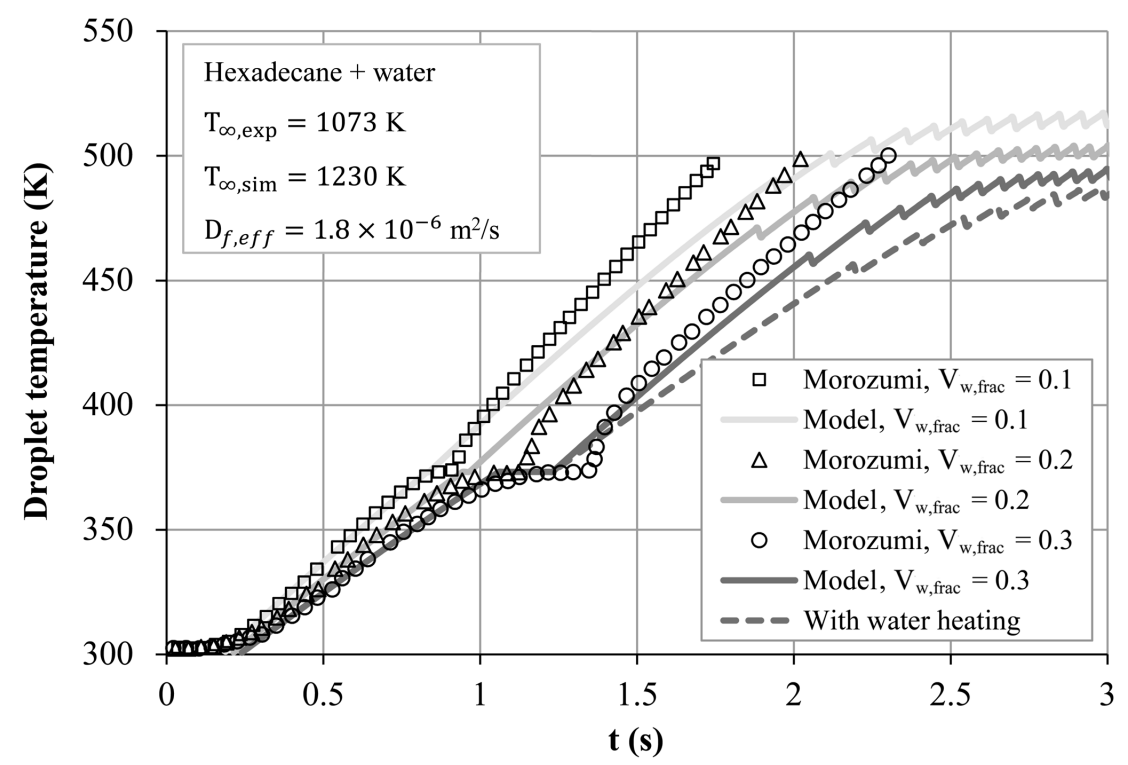

Figure 3.6: Model results of the droplet diameter evolution for water-in-hexadecane emulsion droplets droplets at atmospheric pressure compared against experimental data from Morozumi et al. [37].

temperature after the shell layer is formed. For this heating period, it has been assumed that only the hexadecane increases its temperature while the water remains at its boiling point. This results in a faster heating, as observed in the experiments, although it is still under-predicted. The comparison with a computation where the water is included in the heating is also shown. The same effective water diffusivity value has been used for the three water contents and, as observed in the graph, longer boiling periods are predicted for the higher water contents.

Micro-explosion occurred when the droplet center temperature achieved a temperature of $500 \mathrm{~K}$. As aforementioned, the micro-explosion time can be roughly estimated by the simulation assuming that micro-explosion occurs when the water of the next layer is vaporized (i.e. when the zig-zag behaviour begins). However, the predicted micro-explosion time obtained with this methodology occurs at different temperatures depending on the initial water content. Therefore, another criteria for the occurrence of micro-explosion, like the homogeneous nucleation theory [31], would be necessary for a more precise prediction.

\section{References}

[1] R. I. Mujahid. "Evaporation of liquid fuel droplet". MA thesis. University of Nevada, 2008.

[2] C. K. Law. "Recent advances in droplet evaporation and combustion". In: Progress in energy and combustion science 8 (1982), pp. 171-201. 
[3] G. A. E. Godsave. "Studies of the combustion drops in a fuel spray - the burning of single drops of fuel". In: Fourth Symposium (International) on Combustion. Vol. 4. 1953, pp. 818-830.

[4] D. B. Spalding. "The combustion of liquid fuels". In: Fourth Symposium (International) on Combustion. Vol. 4. 1953, pp. 847-864.

[5] C. K. Law. "Unsteady droplet combustion with droplet heating". In: Combustion and Flame 26 (1976), pp. 17-22.

[6] G. L. Hubbard, V. E. Denny and A. F. Mills. "Droplet evaporation: Effects of transients and variable properties". In: International Journal of Heat and Mass Transfer 18 (1975), pp. 1003-1008.

[7] C. Maqua et al. "Monodisperse droplet heating and evaporation: Experimental study and modelling". In: International Journal of Heat and Mass Transfer 51 (2008), pp. 3932-3945.

[8] B. Abramzon and W. A. Sirignano. "Droplet vaporization model for spray combustion calculations". In: International Journal of Heat and Mass Transfer 32 (1989), pp. 1605-1618.

[9] N. Frössling. "The Evaporation of Falling Drops". In: Gerlands Beiträge zur Geophysics 52 (1938), p. 170.

[10] W. E. Ranz and W. R. Marshall. "Evaporation from drops". In: Chemical Engineering Progress 48 (1952), pp. 141-146.

[11] P. Sierra. "Modelling the dispersion and evaporation of sprays in aeronautical combustion chambers". PhD thesis. Institut Nationale Polytechnique de Toulouse, 2012.

[12] T. B. Gradinger and K. Boulouchos. "A zero-dimensional model for spray droplet vaporization at high pressures and temperatures". In: International Journal of Heat and Mass Transfer 41 (1998), pp. 2947-2959.

[13] H. Zhang. "Evaporation of a suspended droplet in forced convective high-pressure environments". In: Combustion Science and Technology 175 (2003), pp. 2237-2268.

[14] S. K. Aggarwal and H. C. Mongia. "Multicomponent and High-Pressure Effects on Droplet Vaporization". In: Journal of Engineering for Gas Turbines and Power 124 (2002), pp. 248-255.

[15] Y. Yu et al. "Numerical Study on the Characteristics of Vaporization, Ignition, and Turbulent Combustion Processes in Dimethyl Ether (DME)-Fueled Engine Conditions". In: Energy \& Fuels 22 (2008), pp. 3649-3660.

[16] E. W. Curtis and P. V. Farrell. "A numerical study of high pressure droplet vaporization". In: Combustion and Flame 90 (1992), pp. 85-102. 
[17] V. Ebrahimian and C. Habchi. "Towards a predictive evaporation model for multicomponent hydrocarbon droplets at all pressure conditions". In: International Journal of Heat and Mass Transfer, 201054 (2010), pp. 3552-3565.

[18] J.-P. Delplanque and W. A. Sirignano. "Numerical study of the transient vaporization of an oxygen droplet at sub- and super-critical conditions". In: International Journal of Heat and Mas transfer 36.2 (1993), pp. 303-314.

[19] J.-P. Delplanque and W. A. Sirignano. "Transcritical Vaporization and Combustion of LOX Droplet Arrays in a Convectiove Environment". In: Combustion Science and Technology 105.4-6 (1995), pp. 327-344.

[20] P. Haldenwang, C. Nicoli and J. Daou. "High pressure vaporization of LOX droplet crossing the critical conditions". In: International Journal of Heat and Mas transfer 39.16 (1996), pp. 3453-3464.

[21] J. S. Shuen, V. Yang and C. C. Hsiao. "Combustion of Liquid-Fuel Droplets in Supercitical Conditions". In: Combustion and Flame 89.3-4 (1992), pp. 299-319.

[22] R. B. Landis and A. F. Mills. "Effects of internal diffusional resistance on the evaporation of binary droplets". In: Fifth International Heat Transfer Conference. 1974.

[23] C. K. Law, S. Prakash and W. A. Sirignano. "Theory of convective, transient, multicomponent droplet vaporization". In: Symposium (International) on Combustion. 1977.

[24] A. Y. Tong and W. A. Sirignano. "Multicomponent droplet vaporization in a high temperature gas". In: Combustion and Flame 66 (1986), pp. 221-235.

[25] L. F. T. Leite and P. L. C. Lage. "Modeling of Emulsion Droplet Vaporization and Combustion Including Microexplosion Analysis". In: Combustion Science and Technology 157 (2007), pp. 213-242.

[26] P. C. LeClercq, B. Noll and M. Aigner. "Modeling Evaporation and Microexplosion of Water-in-Alkane Emulsion Droplets". In: AIChE Conference Proceedings. 2006.

[27] J. Wilms. "Evaporation of Multicomponent Droplets". PhD thesis. Universität Stuttgart, 2005.

[28] S. V. Ebrahimian. "Development of multi-component Evaporation model and 3D modeling of NOx-SRC reduction system". PhD thesis. Institut National Polytechnique de Toulouse, 2011.

[29] J. Tamim and W. L. H. Hallett. "A continuous thermodynamics model for multicomponent droplet vaporization". In: Chemical Engineering Science 50 (1995), pp. 2933-2942.

[30] N. Doué, P. LeClercq and M. Aigner. "Validation of a multicomponent-fuel droplet evaporation model based on continuous thermodynamics". In: ICLASS. 2006. 
[31] T. Kadota and H. Yamasaki. "Recent advances in the combustion of water fuel emulsion". In: Progress in Energy and Combustion Science 28 (2002), pp. 385-404.

[32] F. L. Dryer. "Water addition to practical combustion systems - Concepts and applications". In: Symposium (International) on Combustion 16.1 (1977), pp. 279295.

[33] S. R. Gollahalli, M. K. Nasrullah and J. H. Bhashi. "Combustion and emission characteristics of burning sprays of a residual oil and its emulsions with water". In: Combustion and Flame 55.1 (1984), pp. 93-103.

[34] J. M. Ballester, N. Fueyo and C. Dopazo. "Combustion characteristics of heavy oil-water emulsions". In: Fuel 75.6 (1996), pp. 695-705.

[35] C. K. Law, C. H. Lee and N. Srinivasan. "Combustion Characteristics of Water-inOil Emulsion Droplets". In: Combustion and Flame 37 (1980), pp. 125-143.

[36] W. B. Fu et al. "A unified model for the micro-explosion of emulsified droplets of oil and water". In: Fuel Processing Technology 79 (2002), pp. 107-119.

[37] Y. Morozumi et al. "A Study of the Occurrence of Microexplosion in a Single Waterin-Oil Emulsion Droplet". In: Journal of Chemical Engineering of Japan 43 (2010), pp. 381-388.

[38] A. Daif, M. Bouaziz and A. A. Chérif. "Comparison of multicomponent fuel droplet vaporization experiments in forced convection with the Sirignano model". In: Experimental Thermal and Fluid Science 18.4 (1999), pp. 282-290.

[39] B. E. Poling, J. M. Prausnitz and J. P. O'Connell. The Properties of Gases and Liquids. McGraw-Hill, 2001.

[40] R.H. Perry and W. Green. Perry's Chemical Engineers' Handbook. McGraw-Hill, 1999.

[41] J. M. Prausnitz, R. N. Lichtenthaler and E. G. Azevedo. Molecular Thermodynamics of Fluid-Phase Equilibria. Pearson Education, 1998.

[42] G. M. Barrow. Physical Chemistry. McGraw-Hill, 1983.

[43] C. Morin et al. "Vaporization and oxidation of liquid fuel droplets at high temperature and high pressure: application to n'alkanes and vegetable oil methyl esters". In: Combustion Science and Technology 176 (2004), pp. 499-529. 


\section{Chapter 4}

\section{Combustion model}

\subsection{Introduction and state of the art}

The distribution of vaporized fuel and temperature - along with the turbulence - in the combustion chamber controls the combustion process: flame shape, flame length, flame temperature, heat release and emissions $[1,2]$. The combustion model in the present work should consider for variations of the equivalence ratio, temperature and water content of the inlet mixture at the specific pressure level. The SCRS model proposed by Pun and Spalding [3] assumes an infinitely fast chemistry and it is based on a single-step stoichiometric reaction. By assuming equal diffusivity for all the species, the mixture fraction is solved with a transport equation and the mass fraction of fuel, oxidant and products are calculated analytically from it. The EBU model developed by Spalding [4] assumes high Reynolds and Damköler numbers, so that the reaction rate depends mainly on the turbulent energy and dissipation rate. This model can also account for chemical kinetics by coupling it with the Arrhenius reaction rate. The laminar flamelet model requires input from experimental data which is used to define the relationships between the temperature, mass fractions and the mixture fraction [5]. The PDF model of Pope [6] is based on the definition of a probability density function, either from experiments or from DNS simulations, that contains the information about the temperature and the mass fractions distributions along with their variances [7]. Although the PDF can have any shape, the presumed PDF model assumes a fixed shape that can be defined with few parameters. In the flame surface density model, the flame is assumed to be locally a stagnation flame and the mean reaction rate is calculated from the product of the flame surface, which depends mainly on the turbulence, and the local reaction rate that is obtained from previously generated tables [7]. Similarly the FGM model developed by Van Oijen and De Goey [8] consists in the tabulation of the internal flame structure (i.e. transport properties, temperatures, mass fractions and progress variable source terms) from detailed laminar flame solutions. 
Simulations of diluted spray flames using the FGM model have been previously performed in $[9,10,11,12]$ for mono-component fuels. In this work a tabulated chemistry model is developed for bi-component fuels, in particular for hydrocarbons with water addition.

\subsection{Model formulation}

The combustion model, developed initially for methane combustion by Dederichs et al. [13], is a combination of the FGM and the SCRS models in order to have a compromise between the computational cost, the size of the tables and the accuracy of the simulations. One dimensional laminar flames are calculated prior to the simulation, using an appropriate kinetic mechanism, and the results are written into tables based on the passive scalars controlling the flame structure. The CFD solver calculates the value of these variables and reads the correspondent flame solution from the tables. Particularly, since the model calculates the mixture composition (from which the temperature is calculated) based on the table variables, it is only required the tabulation of the progress variable source terms. In this way the size of the tables are significantly reduced, especially when many laminar flames are necessary to cover a wide range of conditions.

The present work reformulates the model so that it can simulate the combustion of a generic hydrocarbon $C_{n} H_{m}$, and two variables are added to the chemistry tables - mixture enthalpy and content of a certain species in the fuel - to account for heat losses and to handle bi-component fuels. Additionally, as explained in Chapter 5, the model has been coupled with the lagrangian solver for the simulation of partially vaporized spray flames.

\subsubsection{Table variables}

Table variables are defined in detail because they are used to read the progress variable source term and to calculate the mixture composition.

The first variable of the table is the progress variable, which determines the location of the flame: for a fresh mixture the progress variable is zero while for a burnt mixture it is one. As in the work done by Kuenne et al. [14], the model uses the $\mathrm{CO}_{2}$ product species as the combustion progress variable:

$$
c=\frac{Y_{\mathrm{CO}_{2}}}{Y_{\mathrm{CO}_{2}, e q}}
$$

The second and third variables are employed to specify the mixture composition. These variables are defined by considering that the evaporation model requires a transport equation to be solved for each species component within the liquid droplet, due to the mass transfer of that component from the liquid to the gas phase. Similarly, for each component 
within a gaseous fuel a transport equation is solved. Considering first a hydrocarbonwater-air mixture, these variables are calculated from the fuel and water mass fractions as follows:

$$
\begin{gathered}
Z=Y_{f}+Y_{w} \\
W=\frac{Y_{w}}{Z}=\frac{Y_{w}}{Y_{w}+Y_{f}}
\end{gathered}
$$

The advantage of choosing these variables for the tables, and not $Y_{f}$ or $Y_{w}$ directly, is that they are bounded by zero and one. Therefore, a rectangular matrix can be created for different values of $Z$ and $W$ in order to generate the lookup tables.

The last variable of the table is the enthalpy of the mixture $h$. This table dimension is necessary because of heat losses at the walls, which may be cooled to prevent melting, and also because of the enthalpy non-uniformity upstream of the flame due to the energy employed on the heating and vaporization of the liquid phase.

\subsubsection{Transport equations}

The solved gas phase filtered equations for the transport of mass, momentum, species, progress variable and total enthalpy for compressible flow are presented below:

$$
\begin{aligned}
& \frac{\partial \bar{\rho}}{\partial t}+\nabla(\bar{\rho} \tilde{U})=\dot{\omega}_{M, l a g r} \\
& \frac{\partial \bar{\rho} \tilde{U}}{\partial t}+\nabla \cdot\left(\begin{array}{ccc}
\bar{\rho} & \tilde{U} & \tilde{U}
\end{array}\right)=\nabla \cdot\left(\left(\mu+\mu_{\text {sgs }}\right) \quad \nabla \tilde{U}\right)+\bar{\rho} g-\nabla \bar{P}+\dot{\omega}_{U, \text { lagr }} \\
& \frac{\partial \bar{\rho} \tilde{Y}_{i}}{\partial t}+\nabla \cdot\left(\begin{array}{lll}
\bar{\rho} & \tilde{U} & \tilde{Y}_{i}
\end{array}\right)=\nabla \cdot\left(\frac{\mu+\mu_{\text {sgs }}}{S c} \nabla \tilde{Y}_{i}\right)+\dot{\omega}_{Y, l a g r}
\end{aligned}
$$

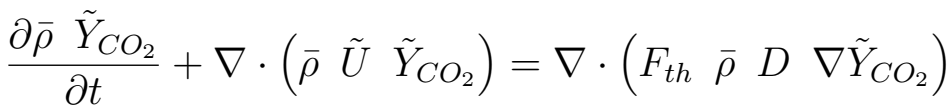

$$
\begin{aligned}
& +\nabla \cdot\left(\frac{\mu_{\text {sgs }}}{S c} \nabla \tilde{Y}_{\mathrm{CO}_{2}}\right)+\Xi^{2} \frac{\alpha}{\alpha+\alpha_{\text {sgs }}} \frac{\dot{\omega}_{\mathrm{CO}_{2}, \text { lam }}}{F_{\text {th }}} \\
& \frac{\partial \bar{\rho} \tilde{h}}{\partial t}+\nabla \cdot\left(\begin{array}{lll}
\bar{\rho} & \tilde{U} & \tilde{h}
\end{array}\right)+\frac{1}{2} \frac{\partial \bar{\rho} \tilde{U} \tilde{U}}{\partial t}+\frac{1}{2} \nabla \cdot\left(\begin{array}{llll}
\bar{\rho} & \tilde{U} & \tilde{U} & \tilde{U}
\end{array}\right) \\
& =\frac{\partial \bar{P}}{\partial t}+\nabla \cdot\left(\left(\alpha+\alpha_{s g s}\right) \nabla \tilde{h}\right)+\dot{\omega}_{h, l a g r}
\end{aligned}
$$

Where $\alpha_{s g s}$ and $\mu_{\text {sgs }}$ are calculated, together with the modelled turbulence kinetic energy, by the selected sub-grid scale turbulence model. The solution of Equation 4.6 is used to calculate the mixture fraction by means of Equation 4.2. The perfect gas equation of state is used by the solver. Thus, deviations from the ideal law are not taken into 
account. Further details on the solved equations, the artificial flame thickening or the flame-turbulence interaction models can be found in [13].

\subsubsection{Calculation of product species mass fractions}

For the combustion of a water-hydrocarbon-air mixture, the following 1-step global reaction is considered:

$$
\begin{aligned}
& C_{n} H_{m}+\nu H_{2} O+\frac{n+\frac{m}{4}}{\phi} O_{2}+\frac{n+\frac{m}{4}}{\phi} \frac{1-b}{b} N_{2} \\
& \rightarrow \epsilon \mathrm{CO}_{2}+\beta C O+(\eta+\nu) H_{2} O+\theta H_{2}+\zeta O_{2}+\frac{n+\frac{m}{4}}{\phi} \frac{1-b}{b} N_{2}
\end{aligned}
$$

where $b$ is the mole fraction of $O_{2}$ in air. The mass fraction of each species in the above equation is calculated analytically from the $\mathrm{CO}_{2}$ mass fraction, $Z$ and $W$. Since the water is considered as a fuel component, the equivalence ratio is calculated using the equation below:

$$
\phi=A F R_{s t_{C_{n} H_{m}}}(1-W) \frac{Z}{1-Z}
$$

where:

$$
A F R_{s_{C_{n} H_{m}}}=\left(n+\frac{m}{4}\right) \frac{M_{O_{2}}+\frac{1-b}{b} M_{N_{2}}}{M_{C_{n} H_{m}}}
$$

As nitrogen is an inert species, the calculation of its mass fraction depends only on $Z$ and $W$ :

$$
Y_{N_{2}}=M_{N_{2}} \frac{Z(1-W)}{M_{C_{n} H_{m}}} \frac{1-b}{b} \frac{n+\frac{m}{4}}{\phi}
$$

Due to the consumption of the hydrocarbon at the flame front, $Y_{C_{n} H_{m}}$ is obtained using the progress variable:

$$
Y_{C_{n} H_{m}}=Z(1-W)(1-c)
$$

To obtain the mass fraction of $C O$ the conservation of carbon atoms is applied:

$$
Y_{C O}=\frac{M_{C O}}{M_{C_{n} H_{m}}} n\left(Z(1-W)-Y_{C_{n} H_{m}}\right)-\frac{M_{C O}}{M_{C O_{2}}} Y_{C O_{2}}
$$

For $\phi<1.2$, it is assumed that there is no generation of hydrogen and $Y_{\mathrm{H}_{2}}=0$. Consequently, the mass fraction of the produced water can be obtained from the conservation of hydrogen atoms:

$$
Y_{H_{2} O}=\frac{M_{H_{2} O}}{2}\left\{\frac{m\left(Z(1-W)-Y_{C_{n} H_{m}}\right)}{M_{C_{n} H_{m}}}-2 \frac{Y_{H_{2}}}{M_{H_{2}}}\right\}
$$


The initial water mass fraction must be added to this mass fraction after the calculation of the remaining species, so that the correct composition is obtained. The mass fraction of $\mathrm{O}_{2}$ is calculated from the conservation of oxygen atoms:

$$
Y_{\mathrm{O}_{2}}=\frac{M_{\mathrm{O}_{2}}}{2}\left\{\frac{2(Z(1-W))}{M_{C_{n} H_{m}}} \frac{n+\frac{m}{4}}{\phi}-2 \frac{Y_{\mathrm{CO}_{2}}}{M_{\mathrm{CO}_{2}}}-\frac{Y_{C O}}{M_{C O}}-\frac{Y_{\mathrm{H}_{2} O}}{M_{\mathrm{H}_{2} \mathrm{O}}}\right\}
$$

For $\phi>1.2$, it is assumed that the available oxygen in the mixture is completely depleted during the combustion process:

$$
Y_{O_{2}}=M_{O_{2}} \frac{Z(1-W)}{M_{C_{n} H_{m}}} \frac{n+\frac{m}{4}}{\phi}(1-c)
$$

The mass fractions of water and hydrogen are therefore calculated from Equations (4.16) and (4.15) respectively. For the case that $W$ is unity, the product species are set to zero since there is no flame solution possible.

Once the mass fraction of each component is known, their thermophysical and transport properties are obtained. The mixture properties are then calculated from the molar average of the single species properties, according to the mixture average approach in [15, 16]. It is worth to notice that the amount of water at the inlet is included exclusively in the $W$ variable, and since it is conserved through the flame it does not affect the calculation of the other species. Thus, any other species which are conserved through the flame (e.g. noble gases, extra nitrogen in the air, carbon dioxide, etc.) can be used in this formulation instead of water - provided that the tables are tabulated for that particular species. Additionally, it is also possible to use the $W$ variable for the combustion of a hydrocarbon-hydrocarbon-air mixtures. The calculation procedure for this type of mixtures is the same as the one described above, with slightly modified equations. It can be found in Appendix D together with its verification and a mesh sensitivity study.

\subsection{Results}

The kinetic mechanism for large alkanes is obtained from Biet et al. [17], where it has been developed and validated against experimental data. It consists of 1.754 reactions and 213 species to model the chemical reactions taking place within the reaction zone. It is employed to generate the 1-D laminar flame solutions for the lookup tables. To verify that the combustion model presented in Section 4.2 reproduces similar results as the reference flame solution, two types of mixtures are considered: hexadecane-water-air and hexadecane-methane-air mixtures. 


\subsubsection{Effect of water content}

Using the aforementioned mechanism, laminar flames are computed using the Cantera software [18] for different fuel fractions and water contents at atmospheric pressure and with an inlet temperature of $T=600 \mathrm{~K}$. The resulting flame speeds are shown in Figure 4.1. The isolines of the flame speed form a $40^{\circ}$ angle with the horizontal axis, resulting from the inlet water being modelled as a component of the fuel and accordingly reducing the hexadecane-air mass ratio. For mixture fractions lower than the stoichiometric fraction at $W=0$, as the water content increases the flame speed gets reduced until no flame solution can be found, i.e. zero flame speed. On the other hand, for higher mixture fractions, the flame speed increases until the stoichiometric conditions are achieved and decreases for higher water contents until extinction.

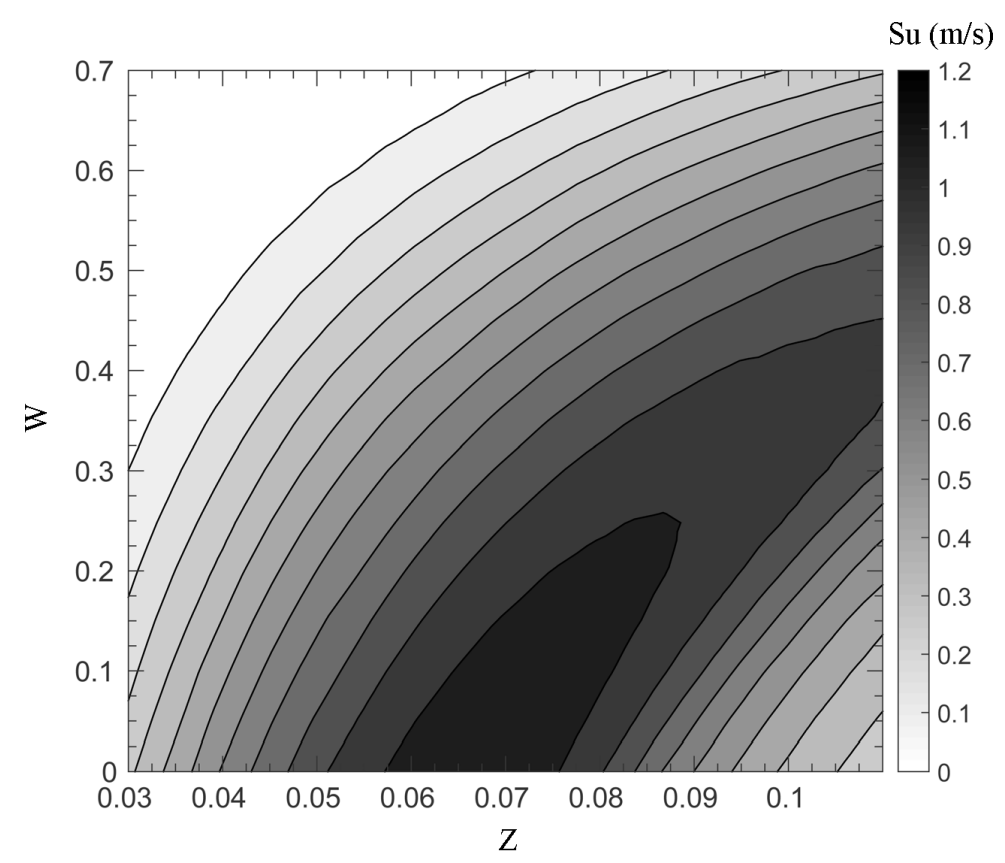

Figure 4.1: Effect of water addition to a hexadecane-air mixture at $\mathrm{P}=1$ bar and $\mathrm{T}=$ $600 \mathrm{~K}$ on the laminar flame speed.

At this point, using these flame solutions, the combustion model assumptions of zero oxygen left for $\phi>1.2$ and negligible hydrogen for $\phi<1.2$ are verified. These are necessary in order to have a closed system of equations for calculating the mixture composition without considering any equilibrium constant. Figure 4.2 shows the hydrogen and oxygen mass fractions at the post-flame region over a range of equivalence ratios for three different water contents. Since the mass fractions are lower than $0.2 \%$ at the relevant region, the assumptions made are adequate for hexadecane-water-air mixtures. These curves are expected to depend mainly on the mixture composition and less on the inlet temperature or on the pressure.

Following this, lookup tables are created and one-dimensional premixed simulations, with a cell spacing of $d x=0.05 \mathrm{~mm}$, are computed for the same $W$ values as in Figure 4.2. 


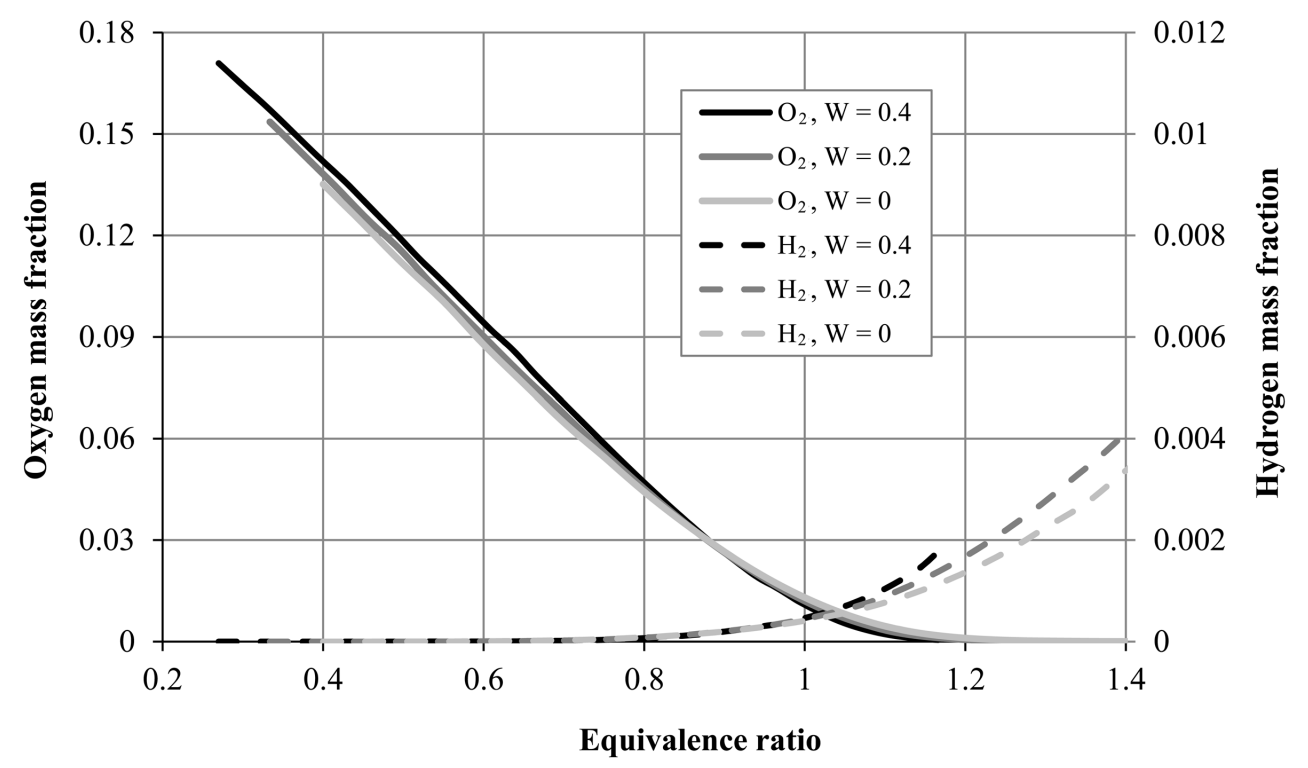

Figure 4.2: Post-flame equilibrium oxygen and hydrogen mass fractions for three different water contents at several equivalence ratios

The laminar flame speed is calculated for each CFD solution using Equation (4.18) and it is plotted in Figure 4.3, which shows that the combustion model is able to reproduce the flame speeds of the reference solution.

$$
S u=\frac{U_{b}-U_{u b}}{\frac{\rho_{u b}}{\rho_{b}}-1}
$$

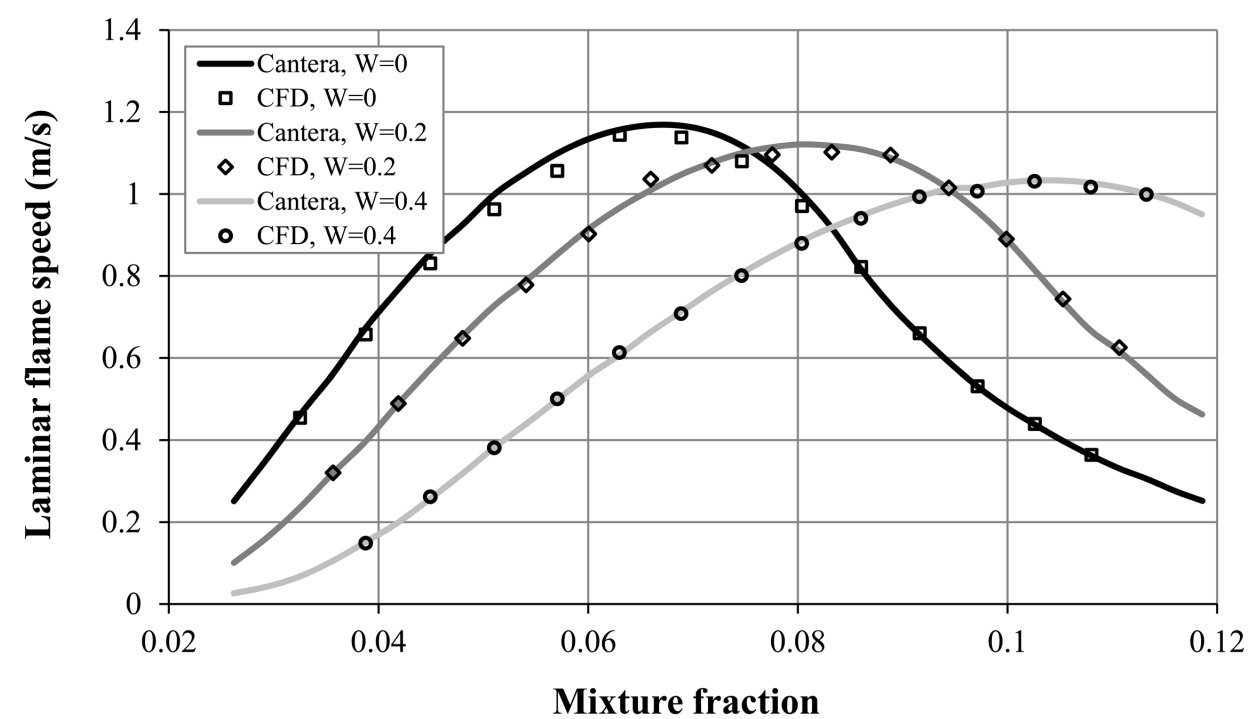

Figure 4.3: Comparison of the laminar flame speed between the CFD model and the reference solution from Figure 4.1.

Mass fractions, temperature, velocity and density profiles are compared with the reference solution in Figures 4.4 and 4.5 for a hexadecane-air mixture close to the stoichiometric conditions. From these figures it can be stated that, with this formulation, 
the peak curve of the $Y_{C O}$ profile is missed by the model and only the equilibrium value is achieved. The use of the progress variable to calculate the hexadecane mass fraction leads to a slower fuel consumption in the post flame region, affecting simultaneously the reduction of $Y_{\mathrm{O}_{2}}$. Regarding the state variable profiles, they are in good agreement with the reference solution.

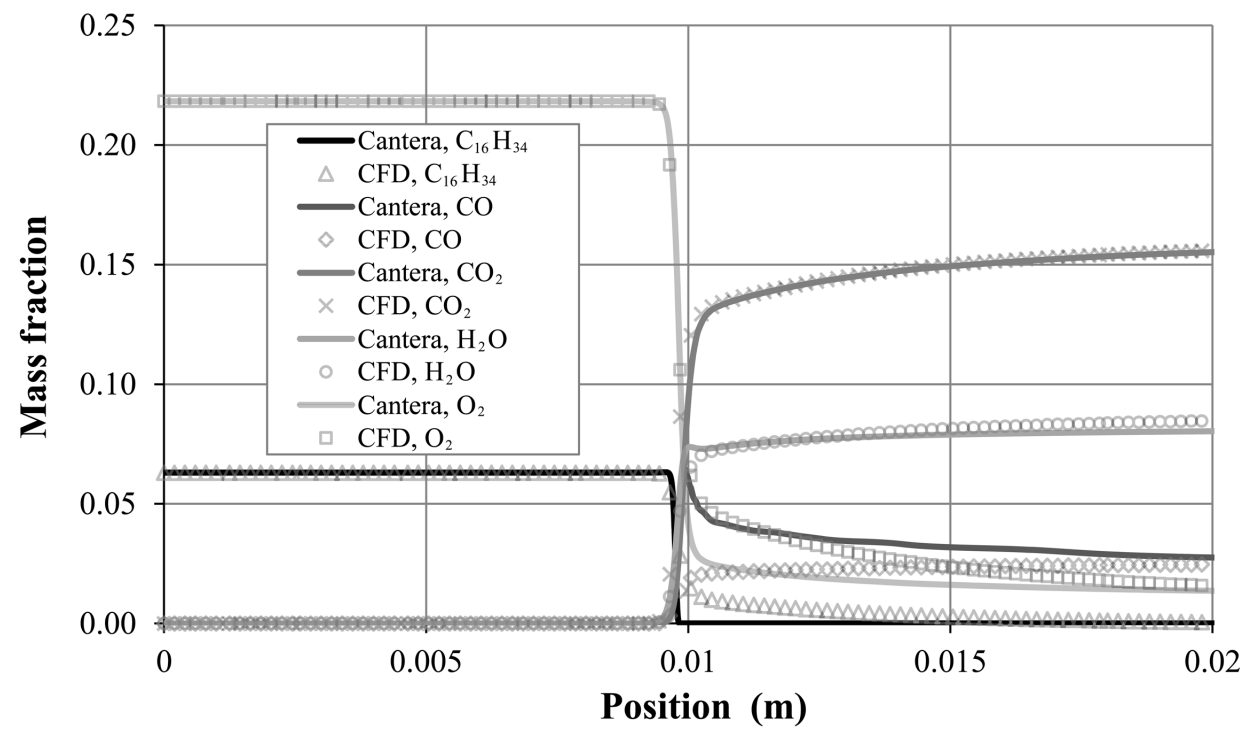

Figure 4.4: Comparison of the species' profiles between the CFD model and the reference solution from Figure 4.1 for $Z=0.063$ and $W=0$.

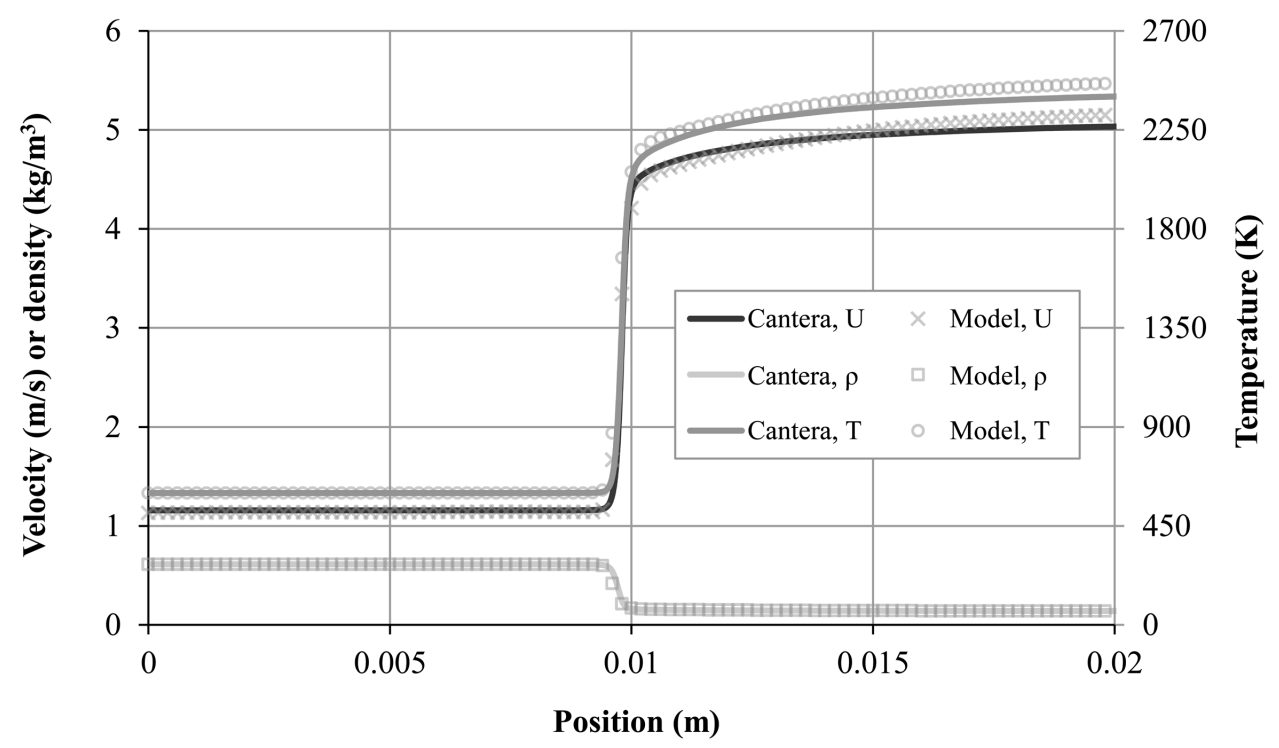

Figure 4.5: Comparison of the temperature, density and velocity profiles between the CFD model and the reference solution from Figure 4.1 for $Z=0.63$ and $W=0$.

In the same way, profiles for a $40 \%$ water content are compared with the Cantera results in Figures 4.6 and 4.7, where initial water content has been added to the water produced in the flame for the CFD solution. As aforementioned, the inclusion of water 


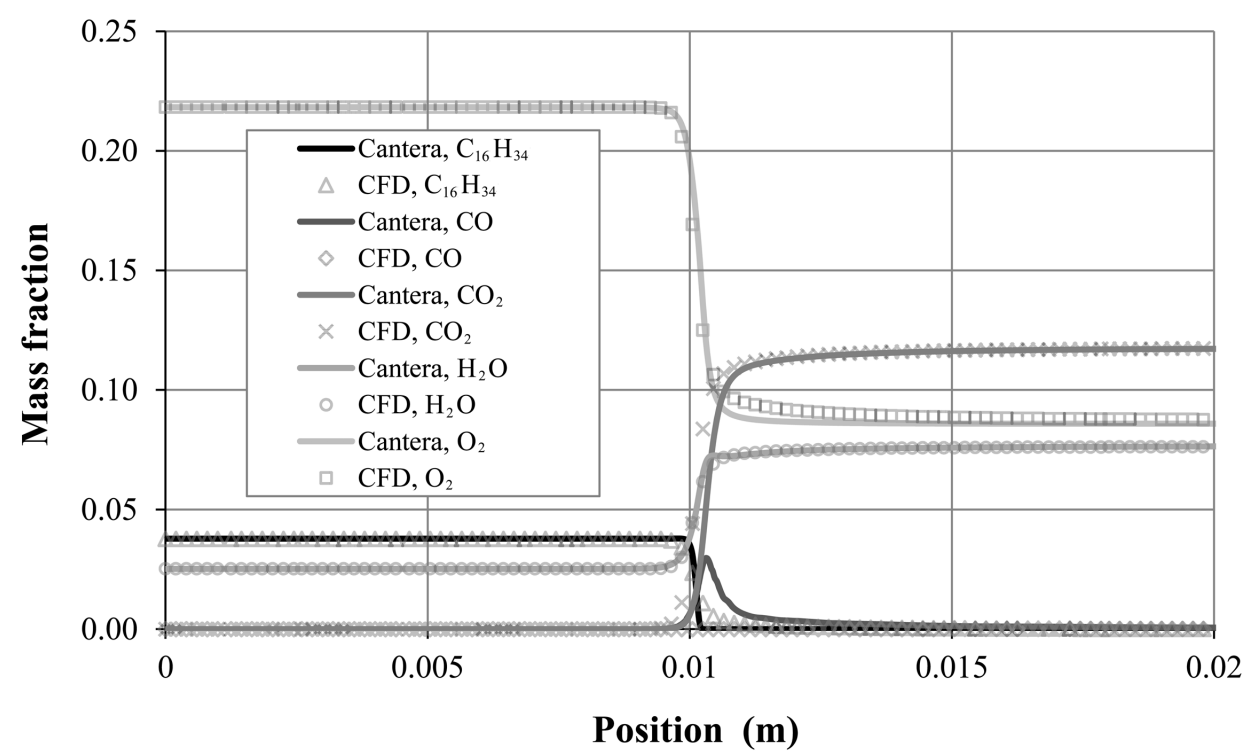

Figure 4.6: Comparison of the species' profiles between the CFD model and the reference solution from Figure 4.1 for $Z=0.063$ and $W=0.4$.

in the $Z$ variable results in profiles characteristics of a leaner mixture when compared to results with $W=0$. In this case, profiles match better the reference solution because the flame is thicker and the progress of $Y_{\mathrm{CO}_{2}}$ is faster than in Figure 4.4. State variables are also properly predicted by the model.

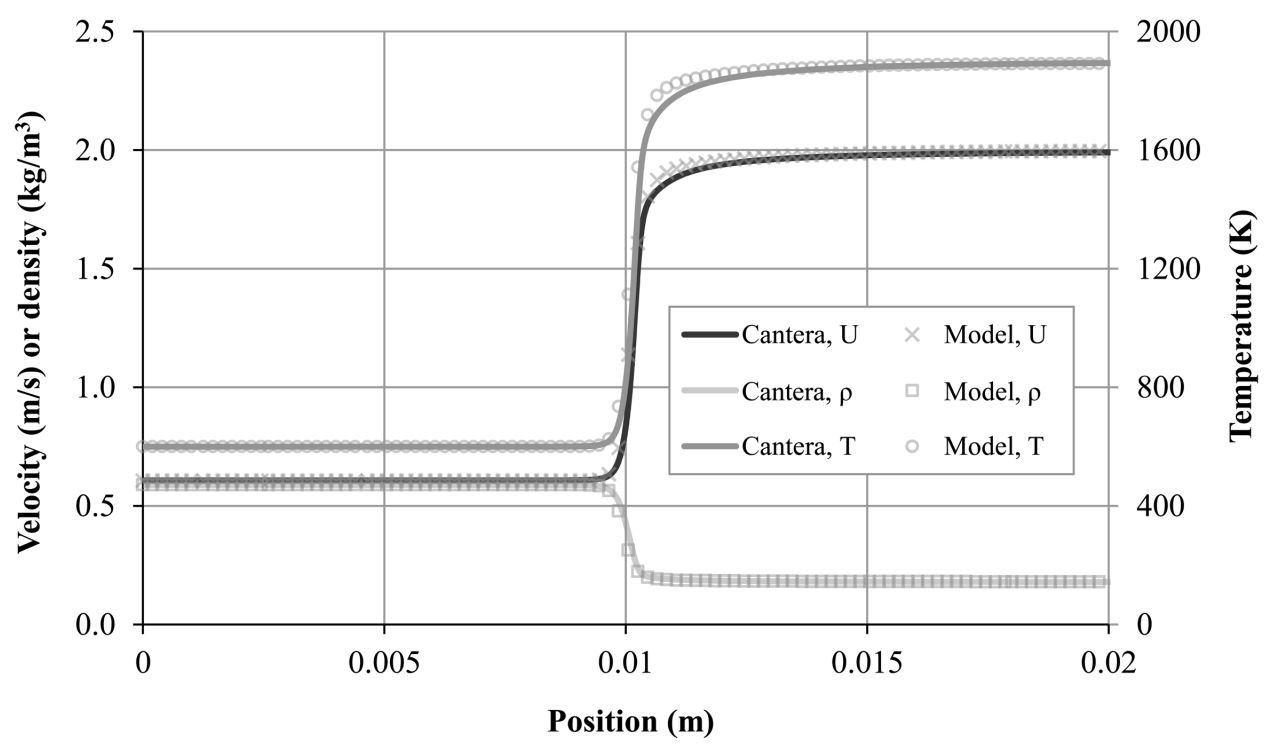

Figure 4.7: Comparison of the temperature, density and velocity profiles between the CFD model and the reference solution from Figure 4.1 for $Z=0.063$ and $W=0.4$.

\subsubsection{Effect of pressure}

No dimension is used in the tables to account for the effect of pressure on the flame speed since no significant pressure variations are expected inside a gas turbine combustion 


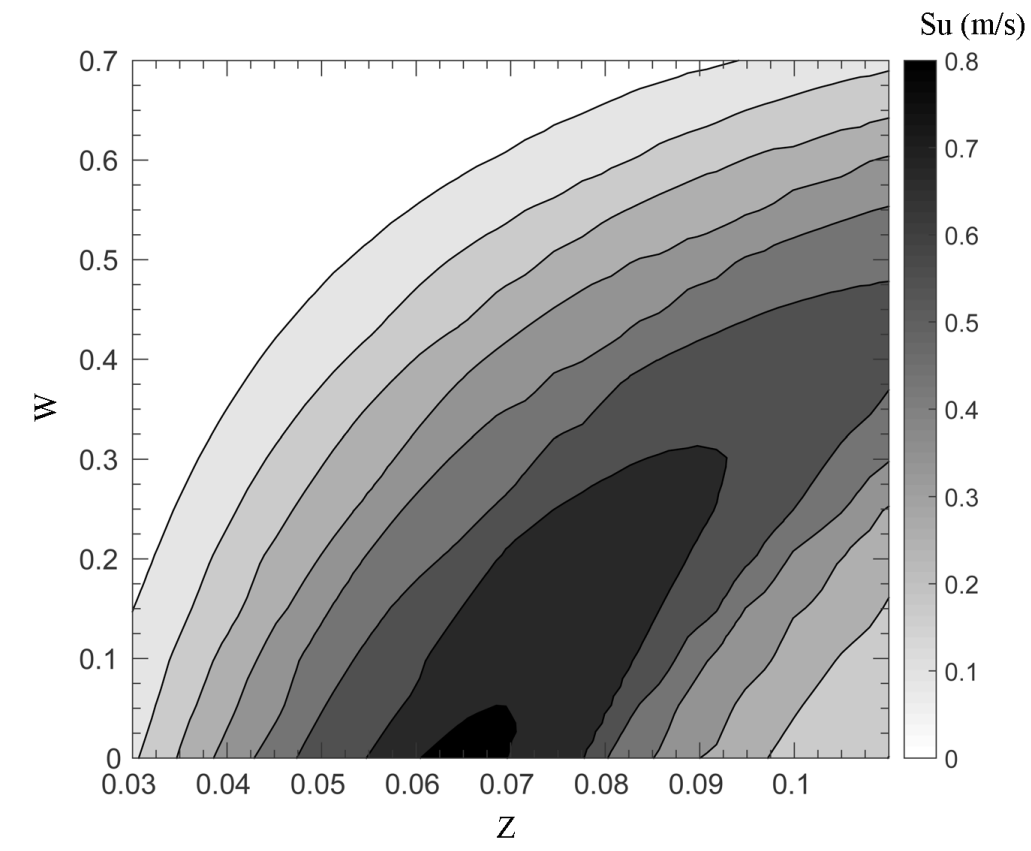

Figure 4.8: Effect of water addition to a hexadecane-air mixture at $P=10$ bar and $T=600 \mathrm{~K}$ on the laminar flame speed.

chamber. However, it must be proved that the model can be used at higher pressures because many different operating points of the combustor may be simulated. Therefore the same 1-D cases as in previous sections (i.e. same inlet mixture compositions and temperature) are computed at $P=10$ bar for comparison.

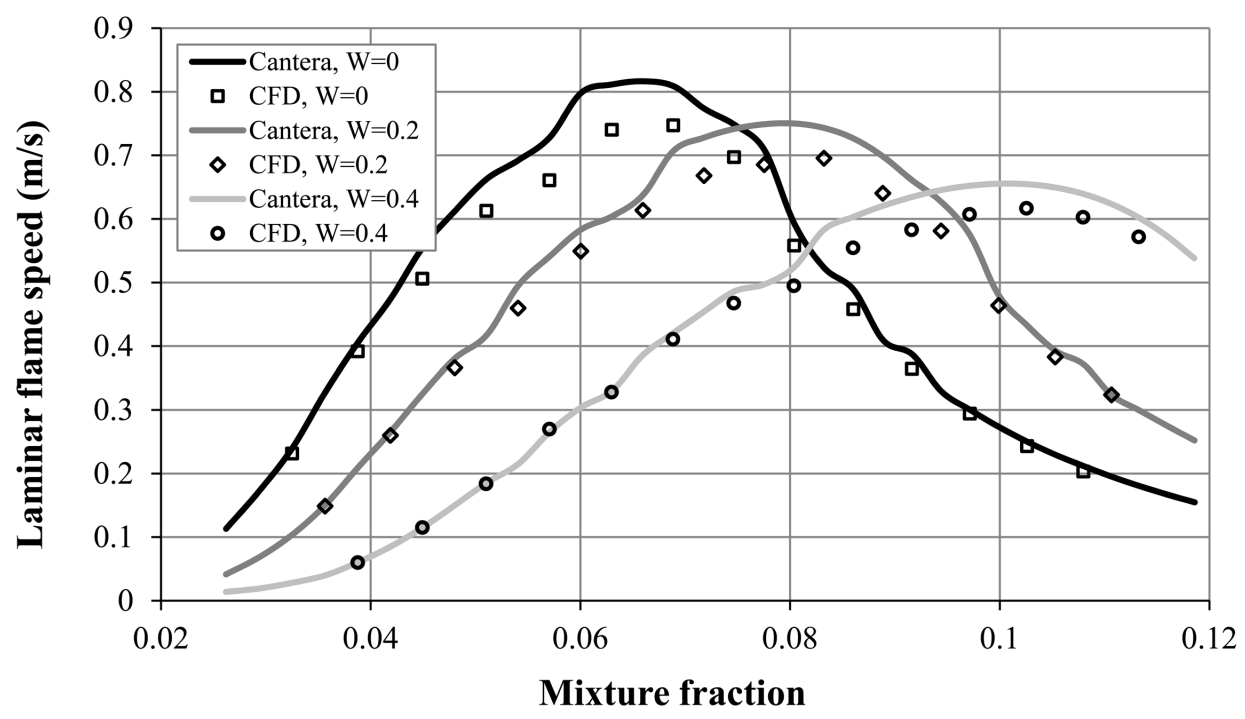

Figure 4.9: Comparison of the laminar flame speed between the CFD model and the reference solution from Figure 4.8 .

Figure 4.8 depicts the laminar flame speeds of a hexadecane-water-mixture using Cantera. When comparing it to Figure 4.1, it is observed that the flame speed is significantly reduced and the area where no solution is found has expanded. The shape of 
the contour lines are not very smooth at higher pressure, but the decrease in flame speed is similar for all water contents. The latter can be observed in Figure 4.9, where the relative position of one curve from the other two is similar to the one for atmospheric conditions. CFD results are also represented in the graph, and the under-prediction of the laminar flame speeds around the stoichiometric point becomes more evident at higher pressures. At rich and lean conditions, however, the flame speeds are still correctly predicted.

A similar analysis is performed for hexadecane-methane-air mixtures in Appendix D, where the calculation procedure of the mixture composition is reformulated because a different global reaction is used.

\section{References}

[1] B. Abramzon and W. A. Sirignano. "Droplet vaporization model for spray combustion calculations". In: International Journal of Heat and Mass Transfer 32 (1989), pp. 1605-1618.

[2] A. H. Lefebvre. Gas Turbines Combustion. Ed. by CRC Press. Taylor \& Francis, 1999.

[3] W. M. Pun and D. B. Spalding. A Procedure for Predicting the Velocity and Temperature Distributions in a Confined, Steady, Turbulent, Gaseous, Diffusion Flame. Aeronautical Research Council, 1967.

[4] D. B. Spalding. "Mixing and Chemical Reaction in Steady Confined Turbulent Flames". In: Symposium (International) on Combustion 13.1 (1971), pp. 649-657.

[5] H. K. Versteeg and W. Malalasekera. An introduction to Computational Fluid Dynamics: The Finite Volume Method. Longman Scientific \& Technical, 1995.

[6] S. B. Pope. "PDF methods for turbulent reactive flows". In: Progress in Energy and Combustion Science 11 (1985), pp. 119-192.

[7] T. Poinsot and D. Veynante. Theoretical and Numerical Combustion. R. T. Edwards, 2005.

[8] J. A. VanOijen and L. P. H. DeGoey. "Modelling of Premixed Laminar Flame using Flamelet-Generated Manifolds". In: Combustion Science and Technology 161 (2000), pp. 113-137.

[9] M. Chrigui et al. "Partially premixed reacting acetone spray using LES and FGM tabulated chemistry". In: Combustion and Flame 159.8 (2012), pp. 2718-2741.

[10] A. Sadiki et al. "Large Eddy Simularions of Diluted Turbulent Spray Combustion Based on FGM Methodlogy: Effect of fuel and Mass Loading". In: Experiments and Numerical Simulations of Turbulent Combustion of Diluted Sprays 19.ERCOFTAC Series (2014), pp. 107-128. 
[11] F. L. Saconamno et al. "LES of a partially premixed spray flame using an adaptativedynamic ATF model coupled to FGM". In: 35th International Symposium on Combustion. 2014.

[12] A. Rittler, F. Proch and A. M. Kempf. "LES of the Sydney piloted spray flame series with the PFGM/ATF approach and different sub-filter models". In: Combustion and Flame 162.4 (2015), pp. 1575-1598.

[13] S. Dederichs, N. Zarzalis and C. Beck. "Validation of a Novel LES Approach Using Tabulated Chemistry for Thermoacoustic Instability Prediction in Gas Turbines". In: ASME Turbo Expo. 2015.

[14] G. Kuenne, A. Ketelheun and J. Janicka. "LES modeling of premixed combustion using a thickened flame approach coupled with FGM tabulated chemistry". In: Combustion and Flame 158 (2011), pp. 1750-1767.

[15] R. Bird, W. Stewart and E. Lightfool. Transport Phenomena. John Wiley \& Sons, Limited, 2013.

[16] C. R. Wilke. "A Viscosity Equation for Gas Mixtures". In: The Journal of Chemical Physics 18 (1950), pp. 517-519.

[17] J. Biet et al. "Experimental and modeling study of the low-temperature oxidation of large alkanes". In: Energy \&6 Fuels 22 (2008), pp. 2258-2269.

[18] D. G. Goodwin, H. K. Moffatt and R. L. Speth. Cantera: An Object-oriented Software Toolkit for Chemical Kinetics, Thermodynamics, and Transport Processes. Version 2.1.2. 2014. 


\section{Chapter 5}

\section{Models coupling}

Individual models for atomization, evaporation and combustion have been presented in the previous chapters. The OpenFOAM implementation has also been verified and, except for the combustion model, validated. To simulate a partially pre-vaporized spray flame, however, these models have to interact between each other. This chapter explains how the models are coupled and simple simulations are performed to analyse this coupling.

\subsection{Atomization-evaporation models coupling}

\subsubsection{Introduction and setup}

Since the atomization and evaporation models are associated to the Lagrangian parcels, they are naturally coupled if both of them are activated in the simulation. No significant temperature rise or evaporated mass is expected from both the jet (elliptical cylinder) and (ellipsoid) parcels, due to their low surface-to-volume ratio. The latter is mainly a consequence of their larger mass, compared to the mass of a secondary droplet.

Even though the evaporation model is developed for a spherical droplet shape, it is also applied to the jet and primary parcel types as a first approach. This implies that the primary droplets evaporate slightly slower than they should, since the surface area of a sphere is minimum for a given volume. For the jet parcels, if their height is in the same order of magnitude as their diameter, they evaporate at a faster rate because the ellipses would not contribute to the parcel's surface area.

For this study, hexadecane is used as a fuel and three different momentum ratios are considered. Simulations with a $60 \%$ water-to-fuel ratio are also performed to investigate the influence of water on the spray evaporation. The same mesh used in Chapter 2 is used and the conditions imposed in each case are included in Table 5.1.

An area-averaged fuel mass fraction is sampled at several axial planes downstream of the injection point. This variable is non-dimensionalized with the fully vaporized and mixed value for a better comparison between the cases. 
Table 5.1: Boundary and injection conditions imposed for the simulation of the evaporating spray cases.

\begin{tabular}{c||ccc|cccc} 
& $T_{\text {in }}$ & $U_{\text {in }}(\mathrm{m} / \mathrm{s})$ & $P(\mathrm{bar})$ & $\dot{m}_{l}(\mathrm{~g} / \mathrm{s})$ & $T_{l, 0}(\mathrm{~K})$ & $Y_{l, w, 0}$ & $q$ \\
\hline \hline dry q6 & 700 & 100 & 8 & 2.152 & 293 & 0 & 6 \\
dry q12 & 700 & 100 & 8 & 3.043 & 293 & 0 & 12 \\
dry q20 & 700 & 100 & 8 & 3.929 & 293 & 0 & 20 \\
\hline wet q6 & 700 & 100 & 8 & 2.263 & 293 & 0.375 & 6 \\
wet q12 & 700 & 100 & 8 & 3.201 & 293 & 0.375 & 12 \\
wet q20 & 700 & 100 & 8 & 4.133 & 293 & 0.375 & 20
\end{tabular}

\subsubsection{Results}

Results for the dry simulations are shown in Figure 5.1. The three curves present an initial heating-up stage where the amount of vaporized fuel increases progressively. This is followed by a more steady evaporation stage where the mixture fraction increases linearly with distance. A higher percentage of the injected mass is vaporized for the lower momentum ratios, despite Figure 2.11 indicates that higher momentum ratios lead to lower global SMD (i.e. volume-to-surface ratio) at the same axial location. Consequently, spray effects in terms of lower temperature and higher fuel concentration around the droplets have a bigger influence than the droplet size.

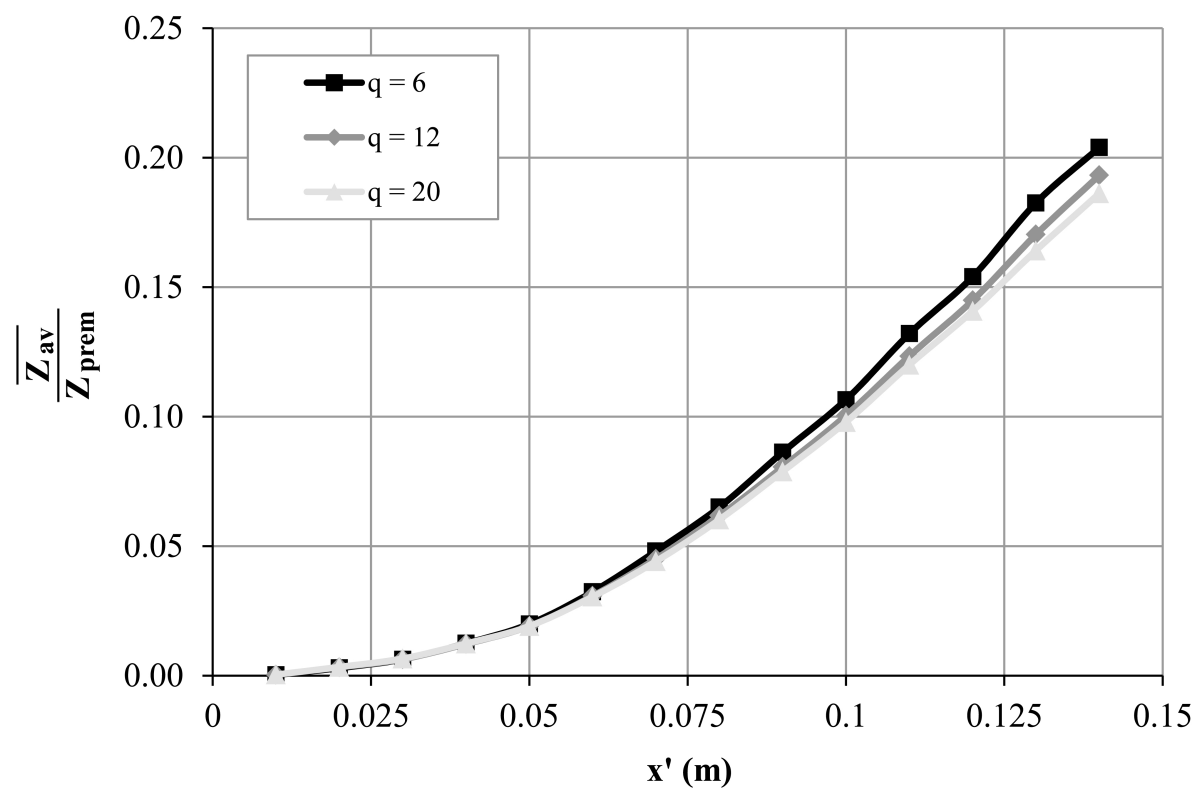

Figure 5.1: Axial evolution of the time- and area-averaged mixture fraction for three evaporating sprays with different momentum ratios.

The mixture fraction profiles for the wet cases, plotted in Figure 5.2, present a higher initial slope due to the greater volatility of water than hexadecane. The water latent heat 
is also higher, which causes a decrement in the gas phase temperature within the spray. This results in a lower amount of vaporized mass than in the dry oil cases at the end of the domain. Figure 5.3 shows the profiles for the time- and area-averaged water content of the vaporized mass. Note that despite the values are lower than the equilibrium, because of the regions that are not affected by the spray, the local values are greater. Particularly, the local water content right downstream of the jet is close to unity. Thus, that graph represents the amount of water vaporized and indicates that a greater percentage of water is vaporized for the larger momentum ratios. This explains the greater differences in the mixture fraction between the three momentum ratios than for the hexadecane sprays.

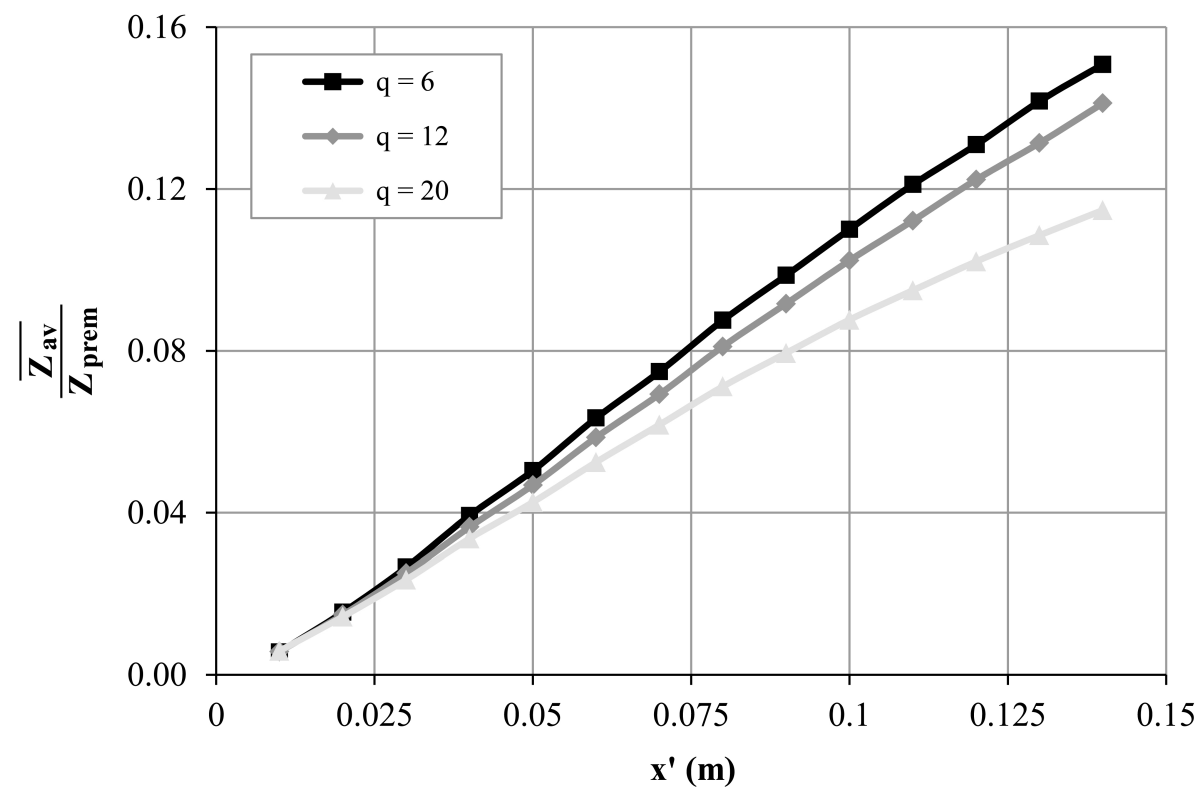

Figure 5.2: Axial evolution of the time- and area-averaged mixture fraction for three evaporating sprays with a $60 \%$ initial water content and different momentum ratios.

\subsection{Evaporation-combustion models coupling}

\subsubsection{Introduction and implementation method}

The coupling between evaporation and combustion models is more complex because it involves two models that are associated to different phases. The spray models are applied to each lagrangian parcel while the combustion model is applied to each cell of the mesh. This sub-section presents the implementation and evaluation of this coupling.

The PIMPLE algorithm [1] is used for the gas phase solver. At the beginning of the outer loop iteration the progress variable source term field, i.e. $\dot{\omega}_{\mathrm{CO}_{2}, \text { lam }}$, is read from the tables and Equations (4.4)-(4.8) are solved. This is followed by the algorithm presented in Section 4.2.3 and the mixture properties as well as the temperature are updated. The outer loop finishes with the inner loop pressure correctors. The time step iteration finishes 


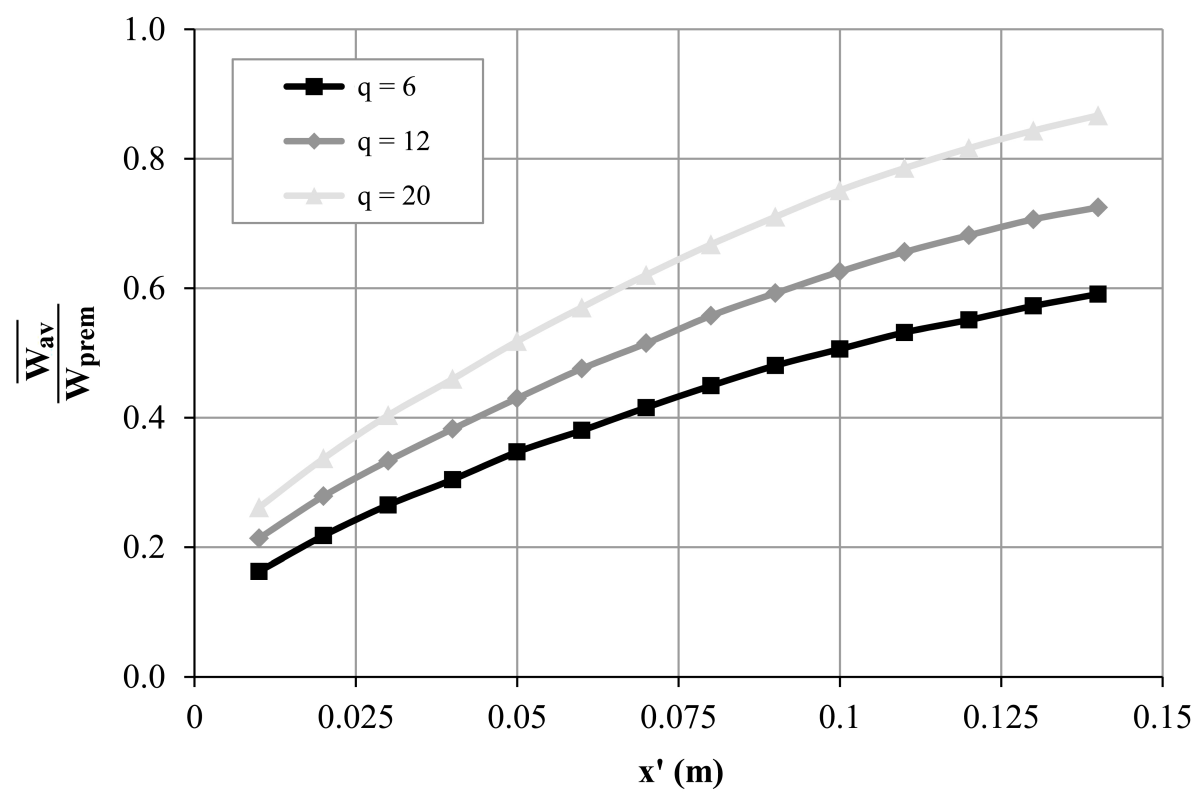

Figure 5.3: Axial evolution of the time- and area-averaged water content of the vaporized mixture for three evaporating sprays with a $60 \%$ initial water content and different momentum ratios.

with the update of the turbulence model variables.

The coupling between the evaporation and combustion models is done via the resolution of the LES governing equations. The spray affects the gas phase enthalpy and fuel species through the source terms in Equations (4.8) and (4.6) respectively, which affect the combustion model. The latter affects, in turn, the spray evaporation by modifying the gas phase temperature, pressure and velocity fields. Consequently, there is no direct coupling between the models but they interact indirectly. Equations (4.2) and (4.3) act as an interface for the fuel species coupling. Furthermore, effect of flame radiation to the droplets is not accounted.

In the case that a droplet crosses the flame front, the interpolated far-field temperature gets significantly increased. This rises the droplet evaporation rate and, apart from enthalpy reduction (accounted in the tabulation), leads to a higher mixture fraction. It is assumed that this increase does not change the equivalence ratio significantly, which applies to the combustion of diluted sprays. The relevant modes of droplet combustion in heavy duty gas turbines were analysed by Beck et al. [3]. For lean mixtures, a greater $Z$ implies an increment in the equilibrium $\mathrm{CO}_{2}$ mass fraction and consequently a larger progress variable source term. Thus, the effect of droplet combustion is partly accounted. For rich mixtures, however, $Y_{C_{2}, e q}$ decreases so that the progress variable becomes negative (but clipped to zero) and the fuel transferred to the gas phase is converted into CO. A similar scenario takes place in the combustion of dense sprays, where the increase in the local mixture fraction is high enough that the $\mathrm{CO}_{2}$ mass fraction does not increase monotonically. For heavy duty gas turbines, lean conditions are typically used and the 
spray is partially vaporized by the time it reaches the flame front. Therefore, the selection of $\mathrm{CO}_{2}$ as a progress variable is assumed to be adequate. Despite the present evaporation model flame does not identify whether a droplet is burning or not, which is likely to affect the evaporation rate, the short lifetime of the droplet after crossing the flame reduces the impact of this assumption.

Additionally, for an emulsion droplet the far-field concentration of water also increases when it crosses flame front. As indicated in Section 4.2.3, the value calculated in Equation (4.15) has to be added to value from the solution of the transport equation for $\mathrm{H}_{2} \mathrm{O}$ and passed to the droplet. As aforementioned, this consideration may be negligible due the short droplet lifetime within the burnt gases region.

\subsubsection{Verification}

The coupling between the evaporation and combustion models is evaluated by performing 1-D spray flame simulations. A 1-D mesh with a constant cell size of $0.05 \times 0.05 \times 0.05 \mathrm{~mm}^{3}$ is used to simulate a laminar spray flame by injecting pure hexadecane droplets with a fixed diameter and with the same axial velocity as the inlet air flow velocity. As aforementioned in Section 3.3, droplets and gas phase are two-way coupled so that drag forces due to non-zero relative velocities are accounted. Since the inlet enthalpy decreases while the fuel mass fraction increases as the droplets are vaporized, the tables for the combustion model are created so that the $h$ and $Z$ ranges - which can determined by performing an initial simulation without combustion - lie within the tables' limits. Laminar flame speeds for the selected ranges are displayed in Figure 5.4, which increase with the mixture enthalpy especially for equivalence ratios close to $\phi=1.1$.

As explained by Jeagle [2], anchored flames are located after the spray is completely vaporized. This means that the coupling between the evaporation and combustion models is weak. These types of flames are typical of diluted sprays, which vaporize before they reach the flame zone. In principle, the flame can be stabilized at any position downstream the evaporation distance, provided that the inlet velocity equals the flame speed. Since the enthalpy at the flame position is initially unknown and the injected fuel mass depends on the amount of inlet airflow, so that the mixture fraction is kept constant, an iterative procedure is used to stabilize the flame. The resulting inlet velocity is independent from the droplet diameter since the same mixture composition and temperature is reached after the evaporation. It is meaningful to have the flame located right after that distance, so that the flame position can be compared to the evaporation distance of a single isolated droplet, which is determined by the evaporation time and the inlet velocity. This comparison is illustrated in Figure 5.12 for a stoichiometric mixture with $\mathrm{T}_{i n, a}=600 \mathrm{~K}$ and $\mathrm{T}_{i n, f}=280 \mathrm{~K}$. After the flame is stabilized, the enthalpy at the flame location is about $h=160 \mathrm{~kJ} / \mathrm{kg}$, which results in a flame speed of $0.95 \mathrm{~m} / \mathrm{s}$. 


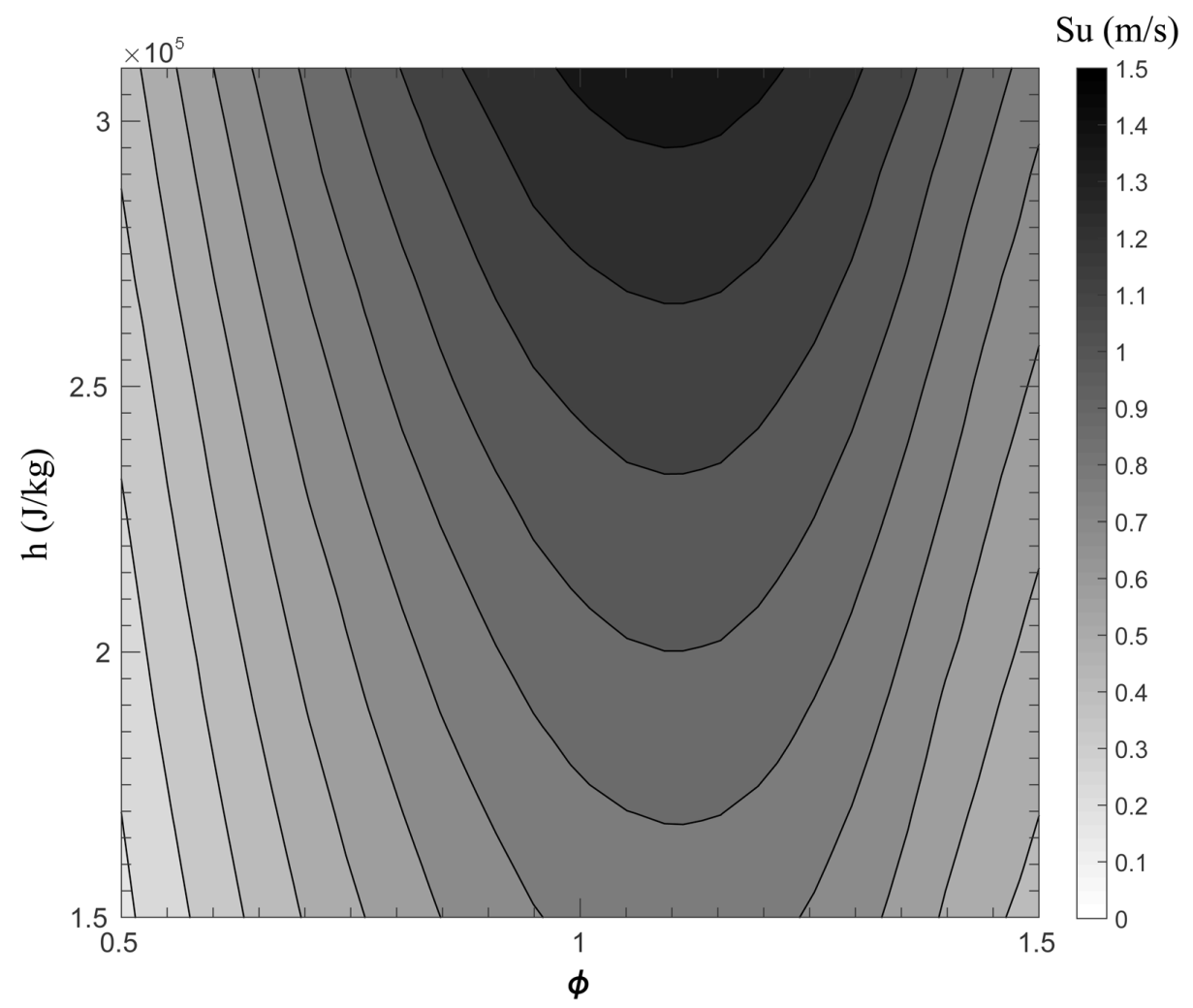

Figure 5.4: Effect of the mixture enthalpy of a hexadecane-air mixture at $P=1$ bar on the laminar flame speed.

The hexadecane mass fraction is displayed in Figure 5.5 for the different initial droplet sizes considered in Figure 5.12. For each case, the mass fraction increases progressively until the stoichiometric fuel-air ratio is reached and it is consumed afterwards. As the droplet size increases, the distance between the particles becomes larger and this results in a oscillatory mass fraction profile. The latter can be observed in Figure 5.6, where the droplet positions and their corresponding diameters are represented. Furthermore, the temperature profile shows that the same gas phase temperature is obtained for all the cases prior to the combustion. Consequently, the same final temperatures and flame speeds are obtained.

On the other hand, saturated flames are located closer to the injection point so that the droplets reach the flame front and get vaporized. This flames are typical of more dense sprays, where the evaporation distance is longer that the pre-mixing distance, so that droplets do not completely vaporize before reaching the flame zone. The interaction between evaporation and combustion is strong because the mixture fraction and enthalpy variables change along the flame. Using the same inlet conditions as for the anchored cases, the flame is initialized to be located slightly upstream the flame position of the anchored flames. Initially, the gain in flame speed due to higher enthalpy at the front of the flame is higher than the loss due to lower fuel fraction. Therefore, the flame travels upstream until the effect of leaner mixture compensates the influence of a higher enthalpy 


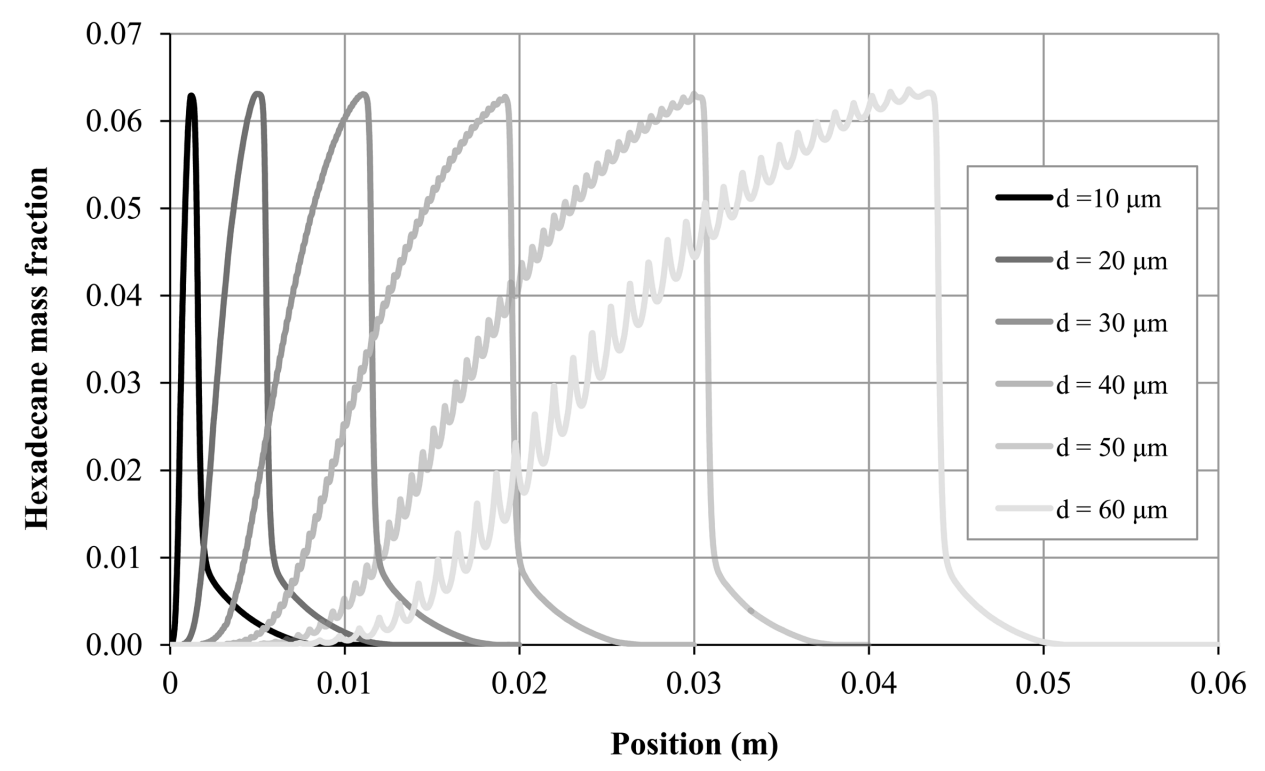

Figure 5.5: Spatial distribution of vaporized hexadecane mass fraction for anchored flames with different initial droplet sizes.

and then it stabilizes.

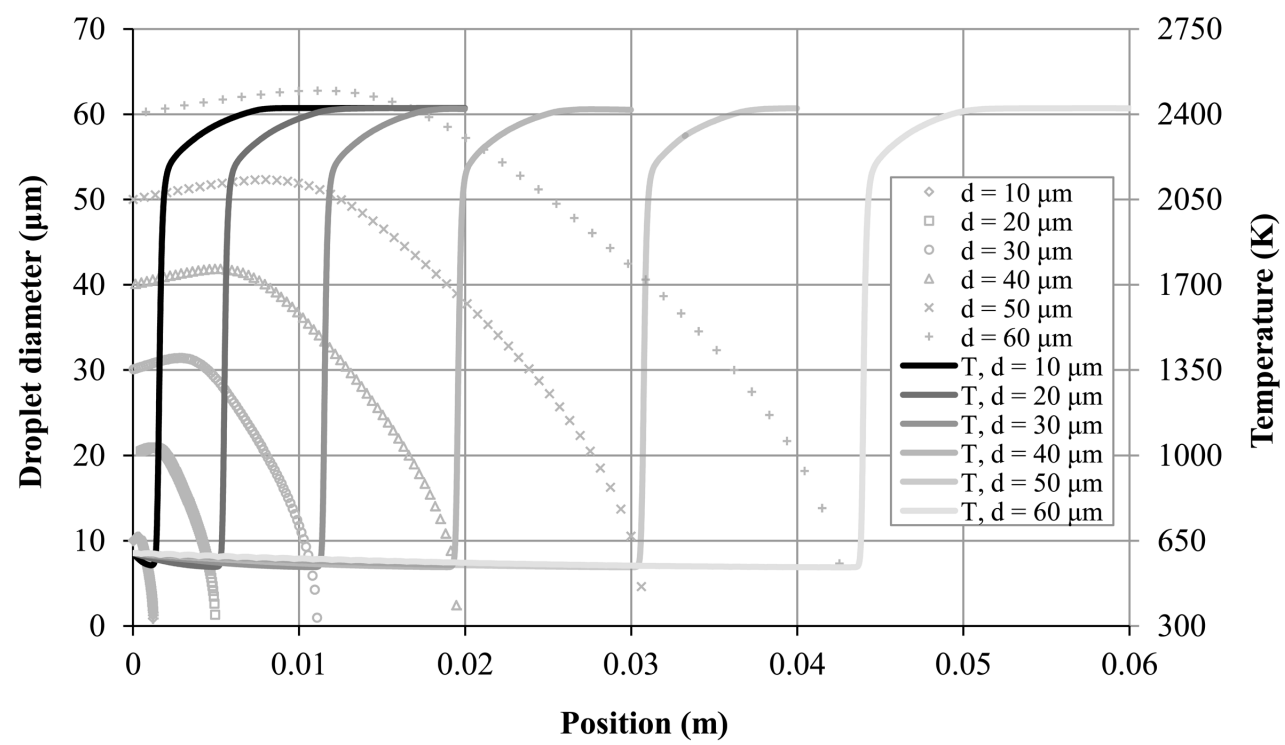

Figure 5.6: Spatial distribution of temperature and droplet diameter for anchored flames with different initial droplet sizes.

Instantaneous profiles for the hexadecane and $\mathrm{CO}_{2}$ mass fractions are depicted in Figure 5.7. It shows that the fuel fraction required to stabilize the flame is higher for the bigger droplet sizes. The peak observed for $d=10 \mu \mathrm{m}$ is due to the fast evaporation of the droplets at the front of the flame, since they are very small and close in distance. As the initial diameter increases, the fuel supply is more intermittent and droplets penetrate further into the burnt mixture. At $d=50 \mu \mathrm{m}$ the droplets start to have an influence $\mathrm{CO}_{2}$ profile, but it still increases monotonically. For $d=60 \mu \mathrm{m}$ the $\mathrm{CO}_{2}$ profile is no longer monotonic due to the mixture fraction gradients within the flame, caused by high 
separation between the droplets and the rapid evaporation of the droplets within the flame. Thus, this case can be considered as the limit where the combustion model can be applied. Temperatures and droplet sizes are plotted in Figure 5.8, which shows how droplets reach the flame before they are completely vaporized and affect the temperature profile by means of a decrease in the mixture enthalpy and an increase in the mixture fraction. A similar final temperature is achieved for all cases and equal to the one in anchored flames, even though the combustion conditions are different, so that the flame speed equals the inlet velocity.

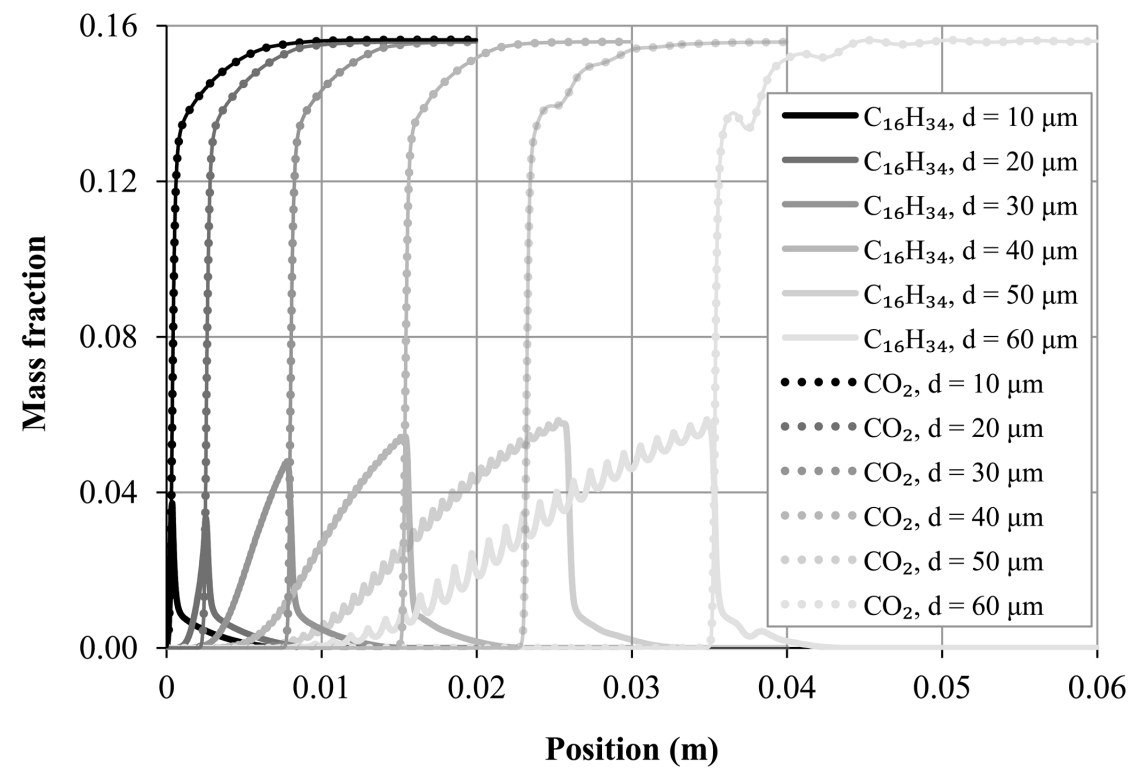

Figure 5.7: Spatial distribution of $\mathrm{CO}_{2}$ and vaporized hexadecane mass fractions for saturated flames with different initial droplet sizes.

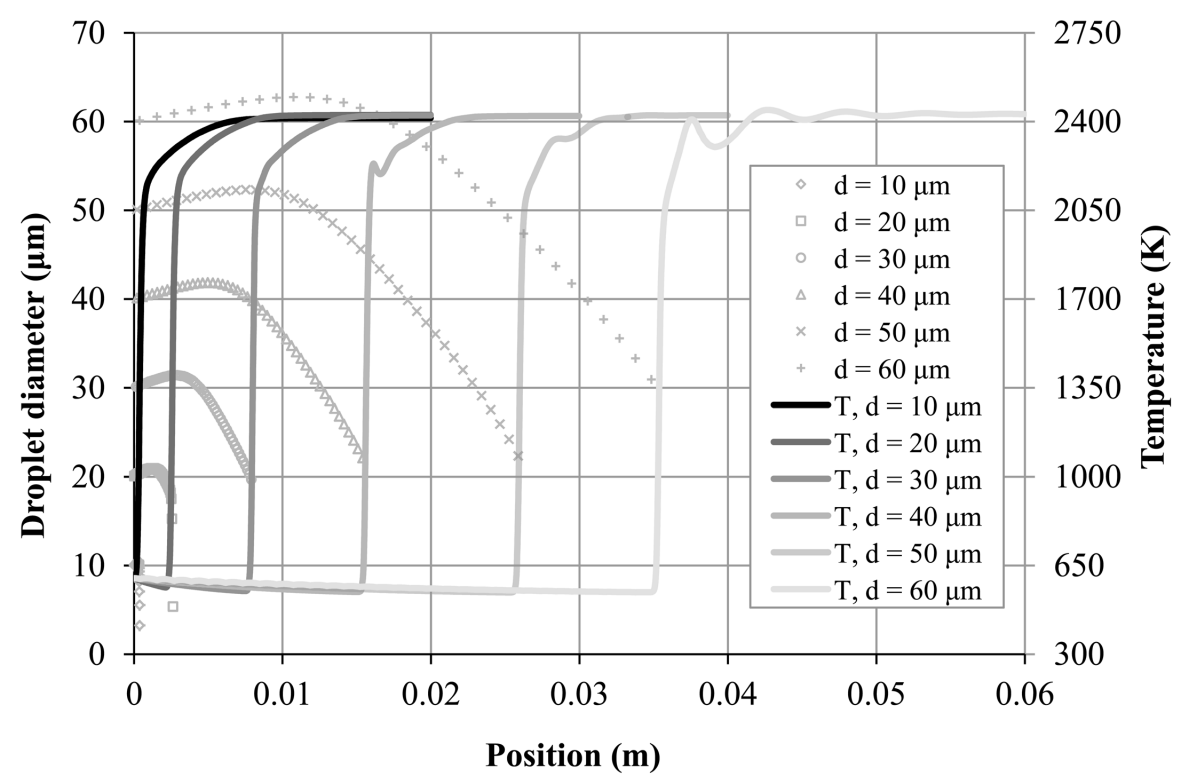

Figure 5.8: Spatial distribution of temperature and droplet diameter for saturated flames with different initial droplet sizes. 
The effect of water addition to the 1-D spray flame solution is also investigated. The hexadecane liquid mass flow from the previous simulations is kept and water is added so that the injected droplets have a $10 \%$ water content. The same parameters for the shell model (i.e. dispersed phase size and effective diffusivity) as in Figure 3.6 are use used. The water content distribution of the vaporized liquid is illustrated in Figure 5.9 for the anchored spray flames. Mostly water evaporates after the injection until a shell is formed. From that point the water content decreases due to the oil evaporation. The depletion of the oil shell is identified by a sudden increase in the water content and a change in the slope. With this model, the next oil shell layer is formed almost immediately.

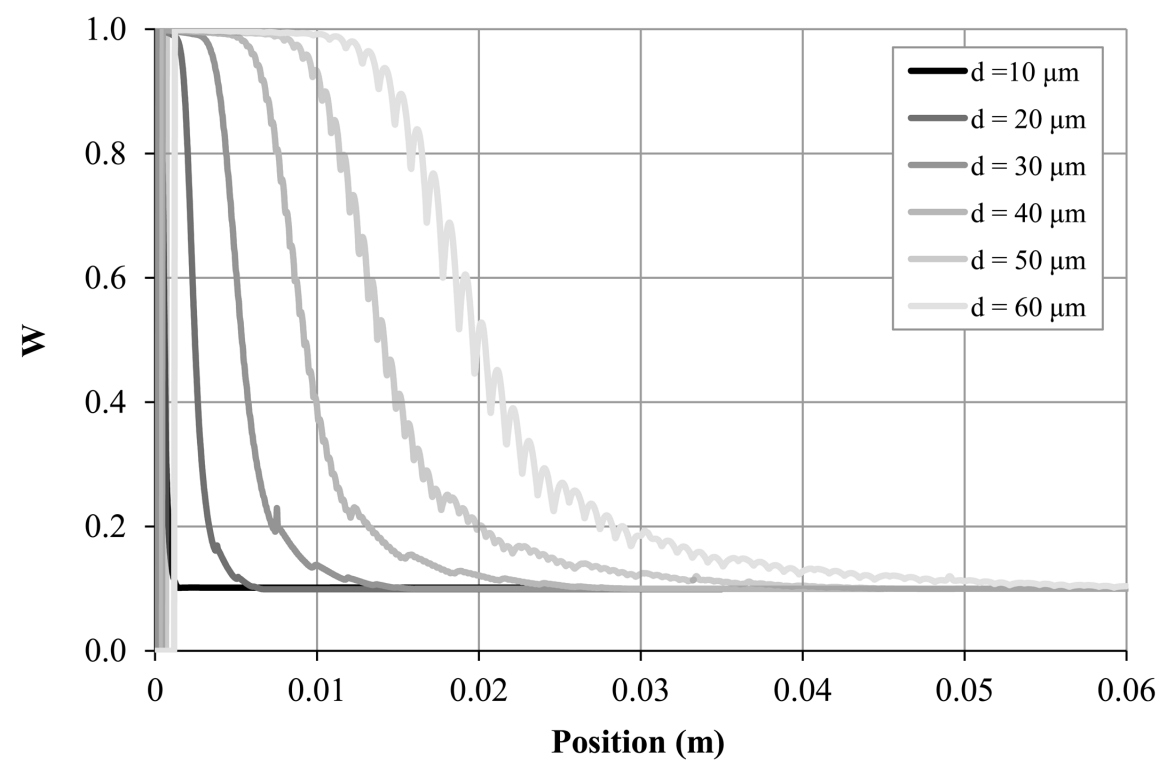

Figure 5.9: Spatial distribution of the water content in the vaporized mixture for anchored flames with $10 \%$ initial water content and different initial droplet sizes.

New chemistry tables are created so that the table variables at the flame position are within the limits during the complete simulation. Particularly for this water fraction, tables have been generated so that $W$ varies from 0.06 to 1.4 in 0.02 intervals, $Z$ from 0.03 to 0.07 in 0.005 steps and $h$ from $45 \mathrm{~kJ} / \mathrm{kg}$ to $155 \mathrm{~kJ} / \mathrm{kg}$ in $10 \mathrm{~kJ} / \mathrm{kg}$ increments. A similar iterative procedure as for $0 \%$ water content is performed to stabilize the anchored flames and a flame speed of $0.92 \mathrm{~m} / \mathrm{s}$ is obtained. Saturated flames are subsequently computed by initializing the flame before the evaporation length. Due to the similarity in the shape of the profiles between anchored and saturated flames, results are shown only for the latter case. The distribution of $\mathrm{CO}_{2}$ and vaporized hexadecane mass fractions are displayed in Figure 5.10. The evolution of $Y_{C_{16} H_{36}}$ is similar to the one in Figure 5.7 but displaced downstream. The level of $Y_{\mathrm{CO}_{2}}$ is higher, which implies that $Y_{C O}$ reduces since the amount of hexadecane is the same. Additionally, the effect of the spray is reduced since the progress variable increases monotonically for all the initial droplet sizes considered. The temperature and droplet size distributions are shown in Figure 5.11. The formation of the first oil layer can be observed due to the presence of a second heating 
stage. The time for the formation of this layer increases linearly with the diameter. Due to the addition of water, a slightly lower temperature values (around $20 \mathrm{~K}$ ) than for the $0 \%$ water case are obtained.

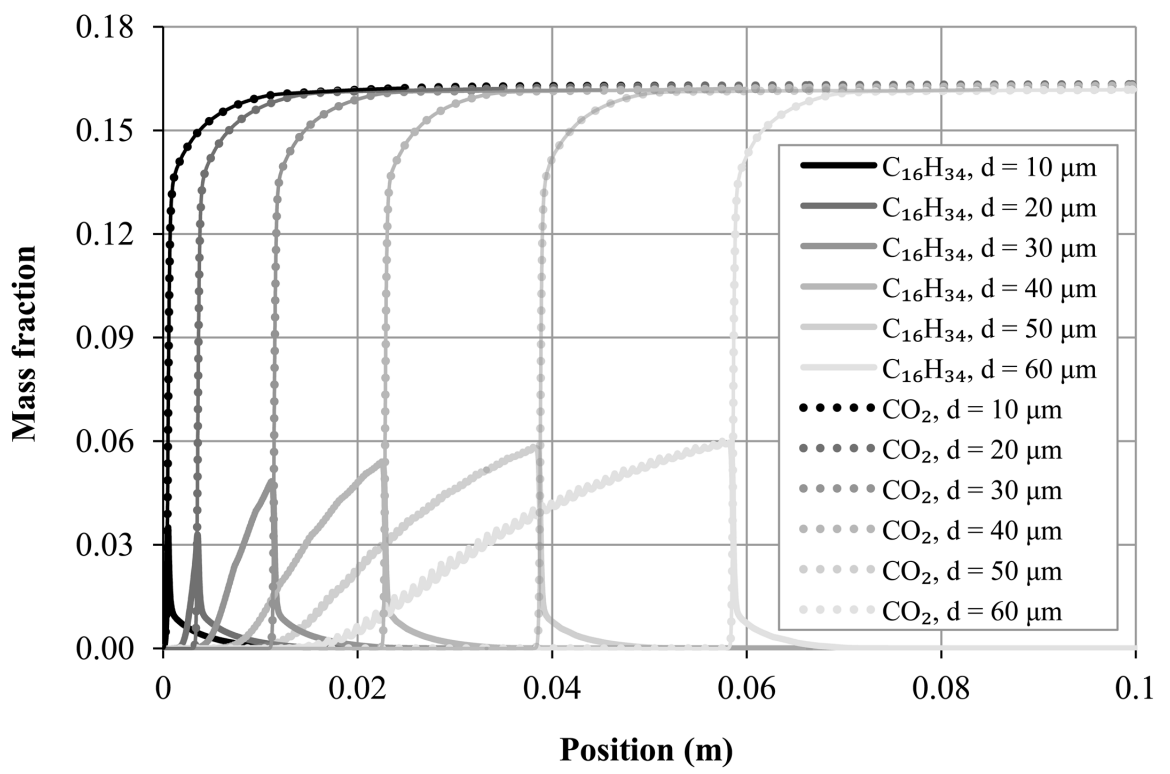

Figure 5.10: Spatial distribution of $\mathrm{CO}_{2}$ and vaporized hexadecane mass fractions for saturated flames with $10 \%$ initial water content and different initial droplet sizes.

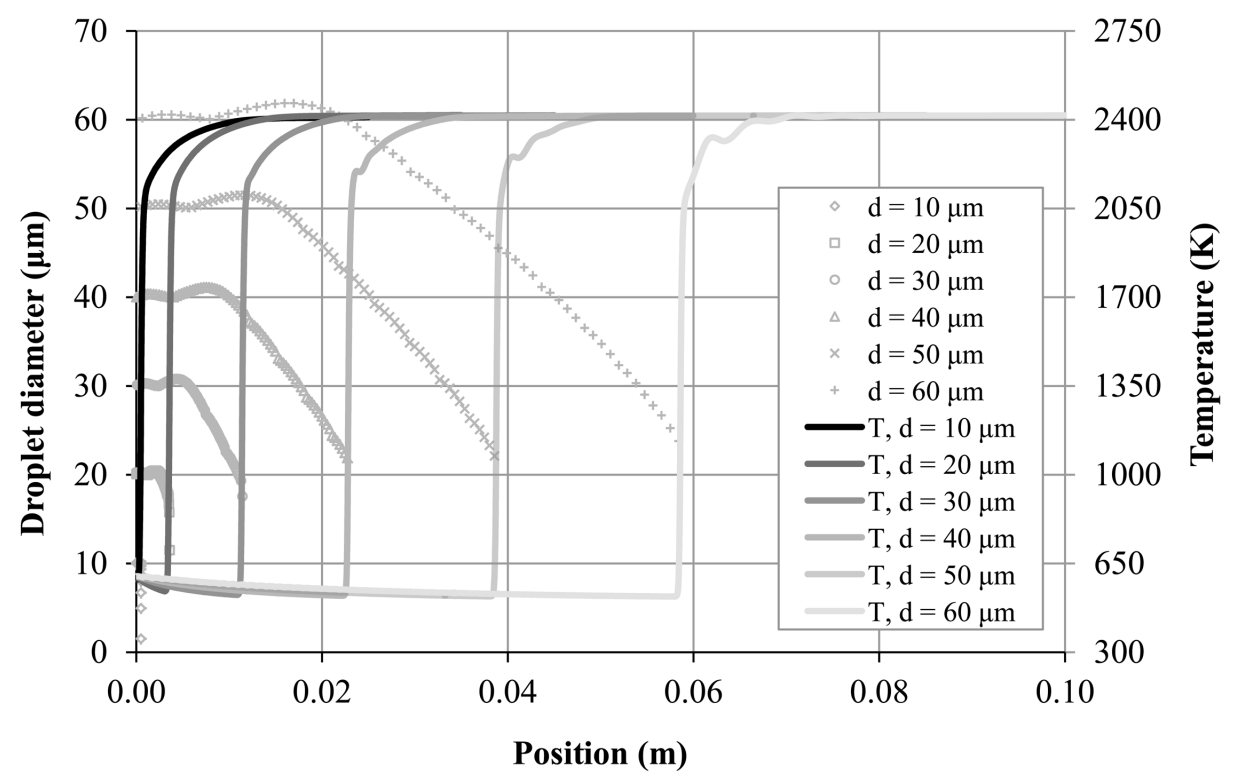

Figure 5.11: Spatial distribution of temperature and droplet diameter for saturated flames with $10 \%$ initial water content and different initial droplet sizes.

The position of the stabilized anchored and saturated flames are plotted in Figure 5.12 for the two water contents considered. The evaporation length of an isolated droplet with the same initial diameter and velocity is also shown. For $0 \%$ water content, spray effects result in an anchored flame position about twice the evaporation distance of an isolated 


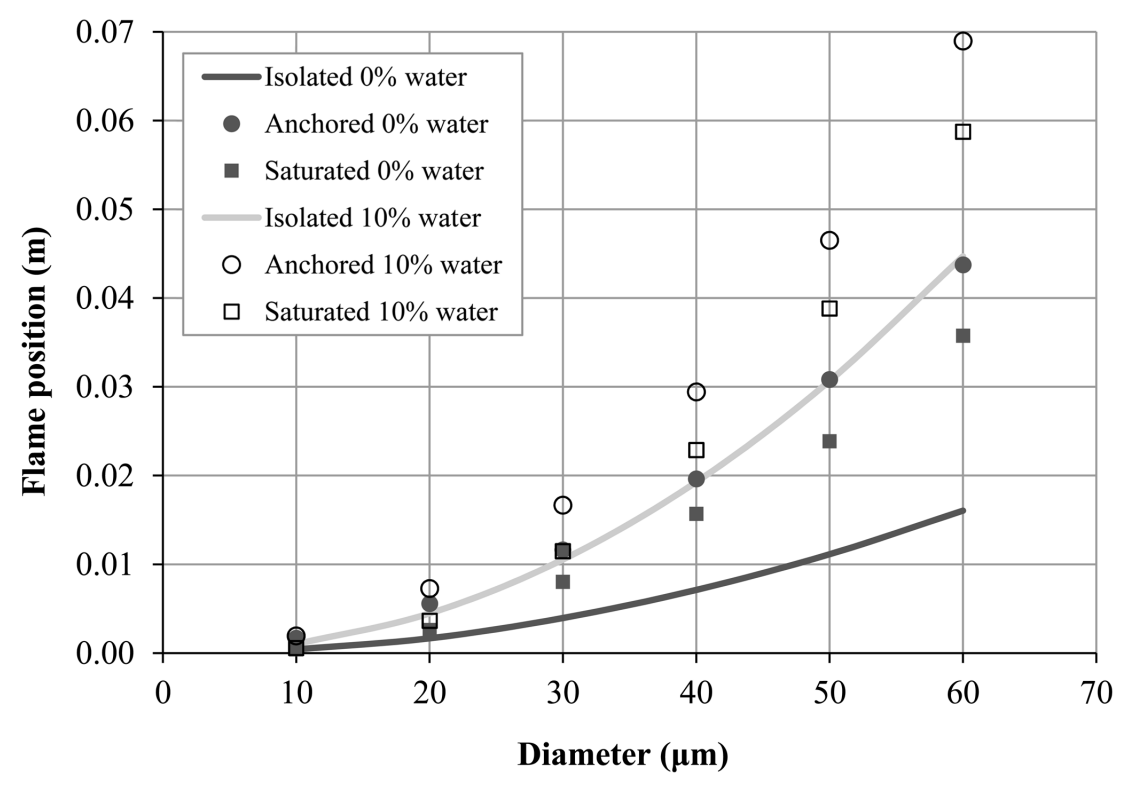

Figure 5.12: Position of the stabilized anchored and saturated flames compared to isolated droplet evaporation length.

droplet. This is due to the reduced temperature and the higher concentration of vaporized fuel. In the case of saturated flames, the distance is similar to the isolated evaporation length for small initial diameters and increases progressively with the droplet size. For the $10 \%$ water content, the evaporation length of an isolated droplet is similar to the position of the anchored spray flame with $0 \%$ water fraction. Therefore, with the spray effects the evaporation length rises further. This increase is slightly lower than in the pure hexadecane case. Furthermore, the distance between anchored and saturated flame positions is higher for the $10 \%$ water case. In particular, for low initial diameters the position of the saturated flame is below the evaporation distance of the corresponding isolated droplet.

\section{References}

[1] H. G. Weller et al. "A tensorial approach to computational continuum mechanics using object-oriented techniques". In: Computers in Physics 12.6 (1998), pp. 620631.

[2] F. Jaegle. "Large eddy simulation of evaporating sprays in complex geometries using Eulerian and Lagrangian methods". PhD thesis. Institut National Polytechnique de Toulouse, 2009.

[3] C. H. Beck, R. Koch and H. J. Bauer. "Identification of Droplet Burning Modes in Lean, Partially Prevaporized Swirl-Stabilized Spray Flames". In: Proceeding of the Combustion Institute. Vol. 32. 2009. 


\section{Chapter 6}

\section{Models' validation for jet flames}

\subsection{Introduction and literature review}

During the development and implementation of new or improved models and algorithms, a validation stage is necessary to ensure that they are adequate for the application investigated. The objective of this process is to gain confidence on the predicted results, to verify that the assumptions made during the model development are acceptable and to identify what are the main modelling gaps that have to be filled in future work. The present chapter focuses on the validation of the tabulated chemistry combustion model for highly turbulent jet flames at pressurized conditions using different fuel mixtures. The latter includes the use of liquid fuels, so that the suitability of the spray models used is also investigated.

Several well-known validation cases are available in the literature. In the TECFLAM burner [1], a swirling flow is created upstream the combustion chamber and the gas fuel is injected co-axially. In the PRECCINSTA project [2], which investigated the mechanisms leading to combustion instabilities in a swirl-stabilized flame, the fuel injection points are located within the guide vanes. The latter is also the case in the scaled Siemens can burner [3], which consists of eight main swirlers and a pilot swirler. Previous validation work on this case with the tabulated chemistry model can be found in Appendix E. Premixed and stratified flames were also investigated by Sweeney et al. $[4,5]$ using two concentric annular ducts, each with a different equivalence ratio, for swirled and non-swirled flows. In the latter case, the flame is stabilized by the recirculation formed at the sides of the jet. This type of flames, also known as jet flames, have also been experimentally investigated in $[6,7,8]$.

This chapter focuses on the simulation of jet flames. Within the context of heavy-duty gas turbine applications, especially for liquid fuel operation, it is preferred to perform the validation at conditions as close to the real engine conditions as possible. Except the scaled Siemens can burner, which has been tested at 4 bar, all the cases mentioned above 
operate at atmospheric pressure. A symmetric jet combustor configuration has been tested by Griebel et. al [9] for pressures up to 14.4 bar. An asymmetric setup has been tested by Lückerath et al. [10] at 20 bar and by Lammel et al. at 8 bar. The latter case consists in three jet flames and it has been simulated with the investigated combustion model by Dederichs et al. [11].

Regarding spray combustion, experimental measurements using acetone and ethanol have been performed by Masri and Gouder [12] at atmospheric conditions, while no relevant data at higher pressures is available in the literature.

The geometry used in the present work is also asymmetric, with only one jet flame, and the pressure level is approximately 8 bar. Three cases are performed with natural gas: one piloted and two non-piloted, with and without propane addition. Three non-piloted cases are performed using liquid fuels: one with dry oil and two with wet oil at different air preheating temperatures. Each simulated case is compared with the experimental data that was measured at the DLR in Stuttgart, Germany.

\subsection{Experimental setup}

The experiments were carried out at the high-pressure combustion test rig of the DLR Institute of Combustion Technology in Stuttgart. This test rig is used for the investigation of scaled combustors at gas turbine relevant conditions. It can be operated with pressures up to 40 bar, a maximum air mass flow of $1.3 \mathrm{~kg} / \mathrm{s}$, air preheat temperatures up to $1000 \mathrm{~K}$, and various gaseous and liquid fuels. The combustor is extensively instrumented with thermocouples, static and dynamic pressure transducers and an exhaust gas analysis. Furthermore the test rig has a large optical access at the combustion chamber, as observed in Figure 6.1, for flame imaging and laser diagnostic techniques.

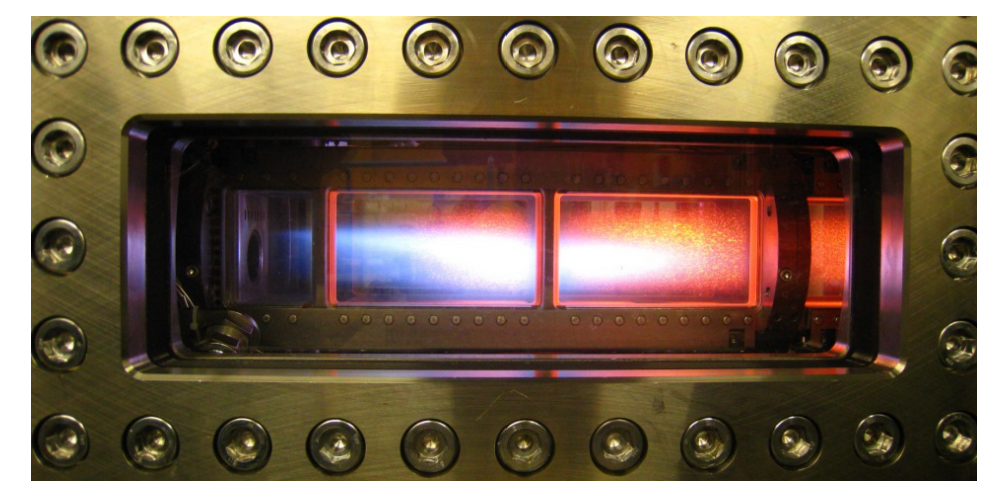

Figure 6.1: Model combustor in operation at DLR in Stuttgart.

The optical measurements conducted to obtain the validation data needed for the numerical simulations are $\mathrm{OH}$-chemiluminescence, particle image velocimetry and laser Mie scattering. An example of a laser diagnostic setup can be seen in Figure 6.2. For the OHCL measurements, the natural emission of the excited $\mathrm{OH}$ radicals at a wavelength of 
approximately $310 \mathrm{~nm}$ is imaged directly with an intensified CCD camera. Note that this is a line-of-sight technique, i.e. the signal is integrated in the image normal direction. 200 images were recorded per operation point with a frame rate of $3 \mathrm{~Hz}$. Image corrections like background subtraction and intensity calibrations were applied and subsequently the image series were averaged, revealing the characteristic flame shape and position by cancelling out turbulent fluctuations.

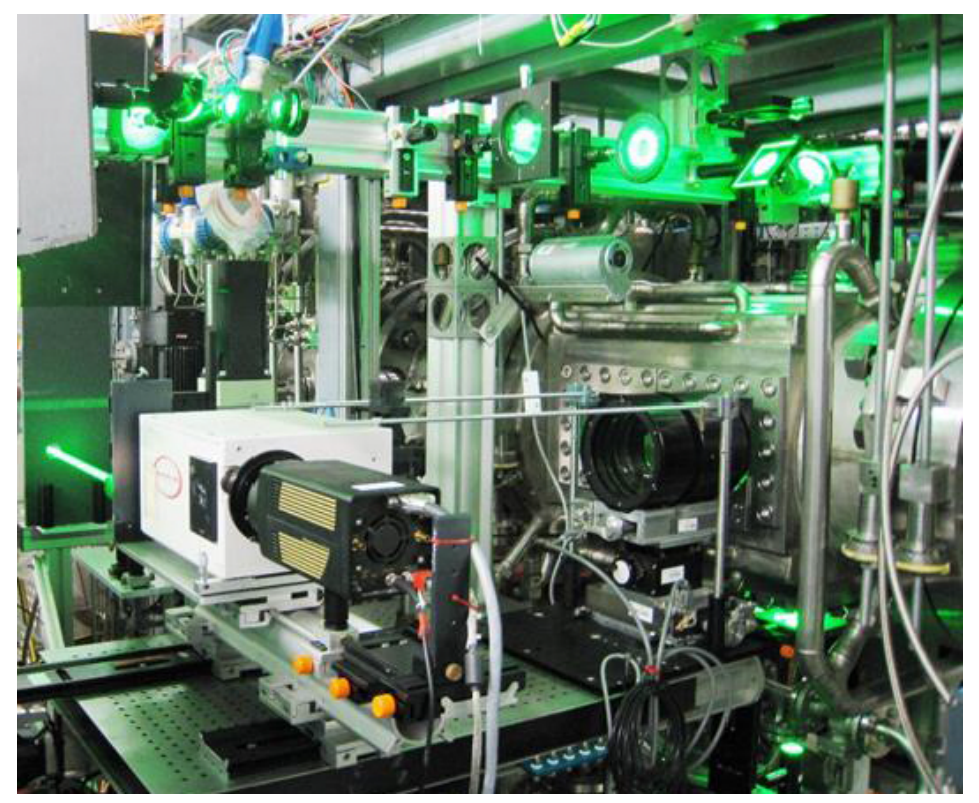

Figure 6.2: Laser diagnostics setup at HBK-S (DLR Stuttgart).

PIV measurements could only be performed for the gaseous fuel cases, where there are no other particles (droplets) in the flow. Small particles of titanium dioxide $(\mathrm{d} \approx 1 \mu \mathrm{m})$ were added to the flow upstream of the combustor and two flashlamp-pumped Nd:YAG lasers $(\lambda=532 \mathrm{~nm})$ were used to illuminate the particles with two consecutive laser sheets. The image pairs of the particle distribution were recorded with a CCD camera $(1600 \times 1200$ pixels) at a repetition rate of $5 \mathrm{~Hz}$. From the image pairs, velocity fields were calculated using a multipass cross correlation algorithm (Software DaVis 8). The spatial resolution for the resulting vector field was approximately $1 \times 1 \mathrm{~mm}^{2} .500$ image pairs were recorded for each measurement and the resulting velocity fields were subsequently averaged in order to obtain the mean flow field and the statistical velocity fluctuations.

For laser Mie scattering, the same experimental setup as for PIV could be used, except there was no seeding of the flow and the laser was only operated in single pulse mode. The laser light is scattered at possibly present droplets of liquid fuel within the combustion chamber, which can be imaged with the same CCD camera, creating an image of the droplet distribution within the flow. This technique is useful to visualize qualitative spray characteristics in the case of liquid fuels. Again, image corrections and statistical averaging were applied to the image series in order to obtain the mean spray distribution within the combustion chamber. 
These results are used to validate the CFD simulations in the scope of this work. Further details on the experimental techniques and additional experimental data are planned for publication by the DLR after the ongoing evaluation.

\subsection{Simulations setup}

The geometry of the computational domain is shown in Figure 6.3. The inlet boundary is located at the plenum, from which the flow is accelerated by means of a conical contraction. Following this, a concentric S-shaped duct leads the air to the central pipe where the fuel is injected and mixed with the air. The premixing duct diameter is $40 \mathrm{~mm}$ and its length is approximately $400 \mathrm{~mm}$. The fuel-air mixture enters a rectangular combustion chamber, with $1 \times 0.1 \times 0.1 \mathrm{~m}^{3}$ dimensions, whose axis offsets vertically from the mixing tube axis by $10 \mathrm{~mm}$ to induce a stable recirculation zone. Seven tubes enter the combustion chamber at the baseplate with a $60^{\circ}$ angle in order to reproduce the effect of a pilot in a real combustion system. These pilot tubes are blocked for non-piloted operation. A nozzle is located at the end of the chamber, which discharges the burnt mixture into a second chamber. This cavity is terminated with a second nozzle where the outlet boundary is placed. The origin of the cartesian axis is located at the center of the baseplate.

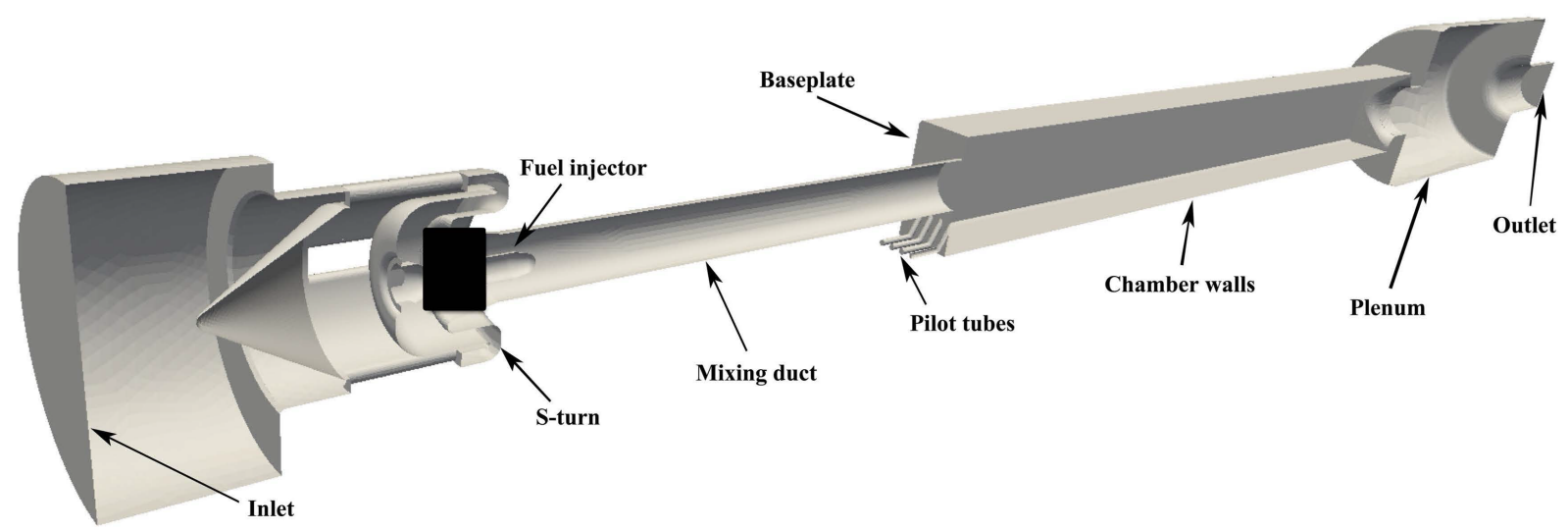

Figure 6.3: Test case geometry. The region around the gas injection is confidential and it has been hidden.

Table 6.1 contains the temperatures, mass flows and fuel compositions of each inlet boundary for the investigated cases. The Baseline case uses only methane as a fuel, and it is taken as the reference case. The only difference with the Piloted case is that the pilot tubes, which operate at stoichiometric conditions, are active. Since the mixture from the premixing passage is lean, the overall equivalence ratio is increased. Regarding the Propane case, it has the same air inlet conditions as the Baseline but the fuel contains $20 \%$ in volume of propane (40\% in mass). A higher fuel mass flow is injected due to the smaller LHV of propane in comparison to natural gas. For the liquid cases, eight circular jets of $0.5 \mathrm{~mm}$ diameter - equally spaced in the circumferential direction - are injected at 
Table 6.1: Conditions imposed at the inlet patches of the simulated cases.

\begin{tabular}{c||ccc|ccc} 
& Baseline & Piloted & Propane & Diesel & Emulsion & Preheated \\
\hline \hline $\mathrm{T}_{\text {in }}(\mathrm{K})$ & 700 & 700 & 700 & 700 & 700 & 800 \\
$\dot{m}_{\text {in }}(\mathrm{g} / \mathrm{s})$ & 538.3 & 538.3 & 538.3 & 538.3 & 538.3 & 468.1 \\
\hline $\mathrm{T}_{\text {inj }}(\mathrm{K})$ & 373 & 373 & 373 & 293 & 293 & 293 \\
$\dot{m}_{\text {inj }}(\mathrm{g} / \mathrm{s})$ & 17.11 & 17.11 & 17.76 & 22.89 & 39.78 & 35.98 \\
Base fuel & $\mathrm{CH}_{4}$ & $\mathrm{CH}_{4}$ & $\mathrm{CH}_{4}$ & $\mathrm{DF} 2$ & $\mathrm{DF} 2$ & $\mathrm{DF} 2$ \\
Fuel additive & - & - & $\mathrm{C}_{3} \mathrm{H}_{8}$ & - & $\mathrm{H}_{2} \mathrm{O}$ & $\mathrm{H}_{2} \mathrm{O}$ \\
$\mathrm{Y}_{\text {additive }}$ & - & - & 0.4 & - & 0.375 & 0.375 \\
\hline $\mathrm{T}_{\text {pilot }}(\mathrm{K})$ & - & 623 & - & - & - & - \\
$\dot{m}_{\text {pilot }}(\mathrm{g} / \mathrm{s})$ & - & 56.95 & - & - & - & - \\
$\mathrm{Y}_{\text {CH }}$, pilot & - & 0.0548 & - & - & - & - \\
\hline $\mathrm{P}_{\text {out }}(\mathrm{bar})$ & 8 & 8 & 8 & 8 & 8 & 8
\end{tabular}

the lance tip into the cross flow with a $40^{\circ}$ angle from the axial direction. The atomization model has been adjusted for this injection angle in Appendix A. For the Diesel case, DF2 is used as a surrogate for diesel fuel. The fuel mass flow is calculated so that a similar primary zone temperature - assuming a fully premixed mixture - as in the Baseline case is achieved. The aim of water addition to the diesel fuel in the Emulsion case is to reduce emission levels, mainly soot and NOx $[13,14,15]$. To ensure a uniform water-diesel ratio distribution at the inlet of the chamber, both liquids are mixed into an emulsion and injected into the cross flow. A higher amount of diesel mass flow compared to the dry case was used to compensate the effect of water on the combustion. Additionally, the evaporation time is greatly affected by the higher latent heat and the lower boiling point of water. The Preheated case reduces the evaporation distance by increasing the air inlet temperature. To maintain the jet velocity and overall equivalence ratio, both the air and fuel mass flows are reduced accordingly.

An important aspect of the simulations is the thermal condition imposed at the wall boundaries. For simplicity, adiabatic conditions are set at the walls located between the air inlet and the baseplate. The heat losses that arise at these walls are accounted for by lowering the inlet air temperature from the experiments by about $25 \mathrm{~K}$. A constant temperature of $700 \mathrm{~K}$ is imposed at the baseplate. For the chamber walls the parabolic profile defined in Equation (6.1) is prescribed.

$$
T_{\text {chamb }}=\min \left(1000,700+\frac{300}{0.09} x^{2}\right)
$$

An adiabatic condition is imposed at the outlet plenum walls as it does not affect the relevant simulation results. Radiation effects are neglected. 
Different meshes are employed for the unpiloted and piloted cases, as observed in Figure 6.4. Both meshes have the same refinements at the inlet geometry, S-duct and injector lance. Both meshes consist of around 8.5 million cells, most of them being hexahedras. The largest cell size, located at the inlet, is $2 \mathrm{~mm}$ while the finest refinement level, located at the S-duct and lance, has a cell size of $0.5 \mathrm{~mm}$. The cell size within the combustion chamber is $1 \mathrm{~mm}$. Additionally, for the piloted case, cylindrical refinements are created for each pilot tube to better resolve the pilot flames and their interaction with the main flame. Since the two outer pilot tubes do no interact with the main jet directly, a higher angle with respect to the axial direction is used. This results in a higher refinement area at the A cut. For the unpiloted mesh, 14 layers have been introduced at the premixing duct walls, being the hight of the first cell about $0.02 \mathrm{~mm}$ and the expansion rate 1.35. This is done to resolve the velocity gradients near the wall, since the Reynolds number at the premixing passage is around 600000. The addition of the layers for the piloted case was more difficult and only a cell refinement (i.e. cell size of $0.5 \mathrm{~mm}$ ) was applied around the premixed duct wall.

The maximum aspect ratio is 62 (6 for the piloted mesh), the maximum skewness is 3.5 and the maximum non-orthogonality is 64 . At the flame region the cells are perfect hexahedra and they have an aspect ratio close to 1 . These meshes fulfil the the quality criteria required by OpenFOAM and they are therefore employed in the simulations, depending on whether the pilot tubes are active or not.

The simulation time step is chosen so that the local CFL number is lower than 10 for the gas simulations, which appear at the gas injection holes, and 4 for oil simulations. The PIMPLE algorithm is applied with 3 outer loops and 2 inner pressure correctors. The Crank-Nicolson scheme is used for temporal discretization. The limitedLinear scheme is selected for divergence terms, while linear schemes are employed for laplacian and gradient terms. After reaching a fully developed reacting flow, simulations are time-averaged for $30 \mathrm{~ms}$ except the Baseline and Piloted cases, which are averaged for $40 \mathrm{~ms}$ because PIV measurements are available.

\subsubsection{Flamelet database}

Chemistry tables are created using Cantera [16] for each case involving a different fuel composition. Tables are generated for several mixture enthalpies to account for the temperature reduction due to fuel heating, evaporation - in case liquid fuels are used - and mixing. Additionally, for the piloted case two fuel-air streams at different eququivalence ratios and temperatures are used. The premixed mixture from the main passage, assuming $30 \mathrm{~K}$ decrease due to fuel mixing, has a higher temperature than the pilot mixture. Thus, tables are generated to cover the enthalpy range from both inlets until the flame position. 


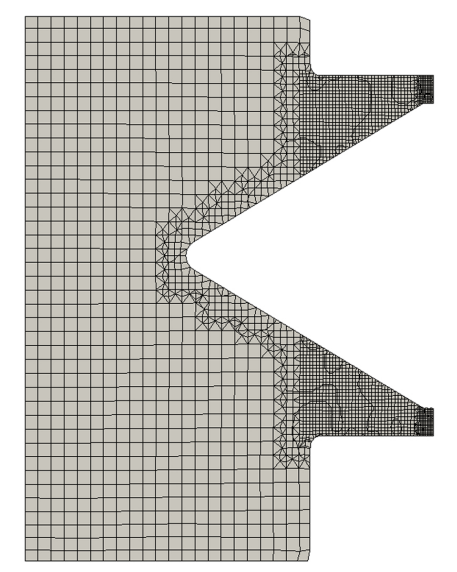

$\mathrm{A} \rightarrow$

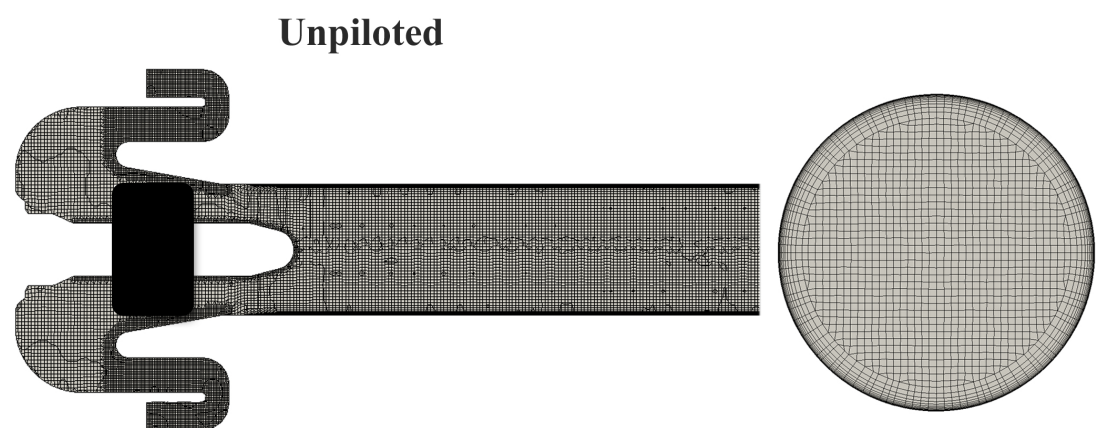

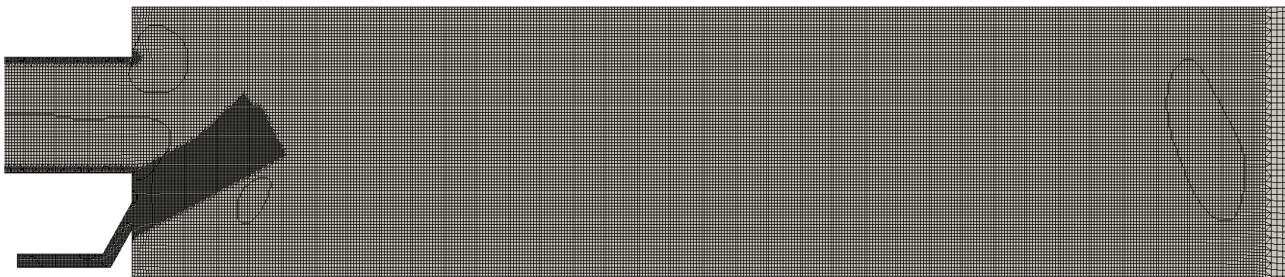

$\mathrm{A} \rightarrow \mathrm{I}$
Piloted

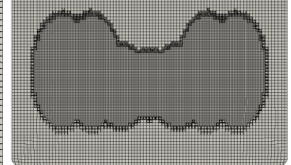

Section A

Figure 6.4: Computational meshes for unpiloted (top) and piloted (bottom) configurations. For the unpiloted cases, the mesh at the combustion chamber corresponds to the bottom image without the pilot refinement. For the piloted case, the mesh upstream of the chamber is the same as the top image with mesh refinements at the premixing duct instead of layers.

The GRI30 mechanism [17], which contains 53 species and 325 chemical reactions, is employed for the cases that have $\mathrm{CH}_{4}$ as the base fuel. Since the additives are well mixed with the base fuel, and their diffusivity in air is assumed to be the same, tables for the Propane case are created only at the specific fuel injection composition. The total enthalpy is reduced from the inlet air enthalpy, which is about $440 \mathrm{~kJ} / \mathrm{kg}$ for a temperature of $700 \mathrm{~K}$, to $-100 \mathrm{~kJ} / \mathrm{kg}$ in $20 \mathrm{~kJ} / \mathrm{kg}$ steps. For each enthalpy, the equivalence ratio has been varied from 0.3 to 1.2 in 0.05 intervals.

For the combustion of a gaseous diesel-water-air mixtures the mechanism from Biet [18] for hexadecane, which considers 1.754 reactions and 213 species, is used for the generation of the chemistry tables from 1-D flame solutions. In this case, the enthalpy of the mixture is reduced from the inlet enthalpy to $-440 \mathrm{~kJ} / \mathrm{kg}$ in $30 \mathrm{~kJ} / \mathrm{kg}$ steps. For each enthalpy, the water content $W$ is varied from 0 to 0.8 in 0.05 intervals, and for each of those cases the mixture fraction $Z$ ranges from 0.026 to 0.104 in 0.06 intervals.

Heat losses at the baseplate and chamber walls are modelled by imposing a thermal wall condition. This results in a low enthalpy of the burnt mixture at the chamber walls which can in turn affect the flame. If the local enthalpy falls below the lowest enthalpy tabulated, the progress variable source term is set to zero to model the flame quenching due to heat losses. 


\subsection{Results}

\subsubsection{Gas fuel}

Firstly, gas fuel configurations are simulated to verify that both the simulation setup and the combustion model produce acceptable results. For this, the simulation results are compared to the PIV and OHCL experimental data.

\section{Unpiloted case results}

The instantaneous and time-averaged axial velocity distribution at a longitudinal and transversal section of the premixing duct is plotted in Figure 6.5. Large turbulent scales can be observed, which are mainly originated by the flow acceleration and turning within the S-duct as well as the area change due to the presence of the lance.

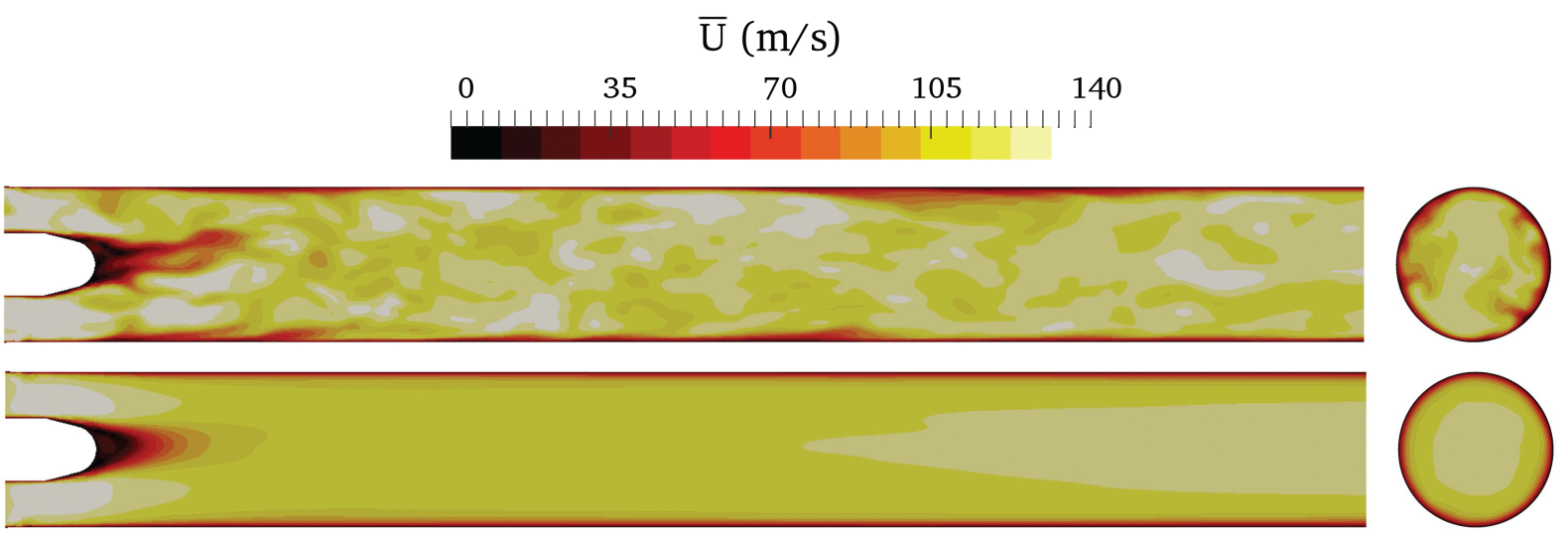

Figure 6.5: Instantaneous axial (top) and averaged (bottom) velocity field of the Baseline case at at a mid-plane within the premixing duct. The axial contours are located $50 \mathrm{~mm}$ upstream of the chamber baseplate.

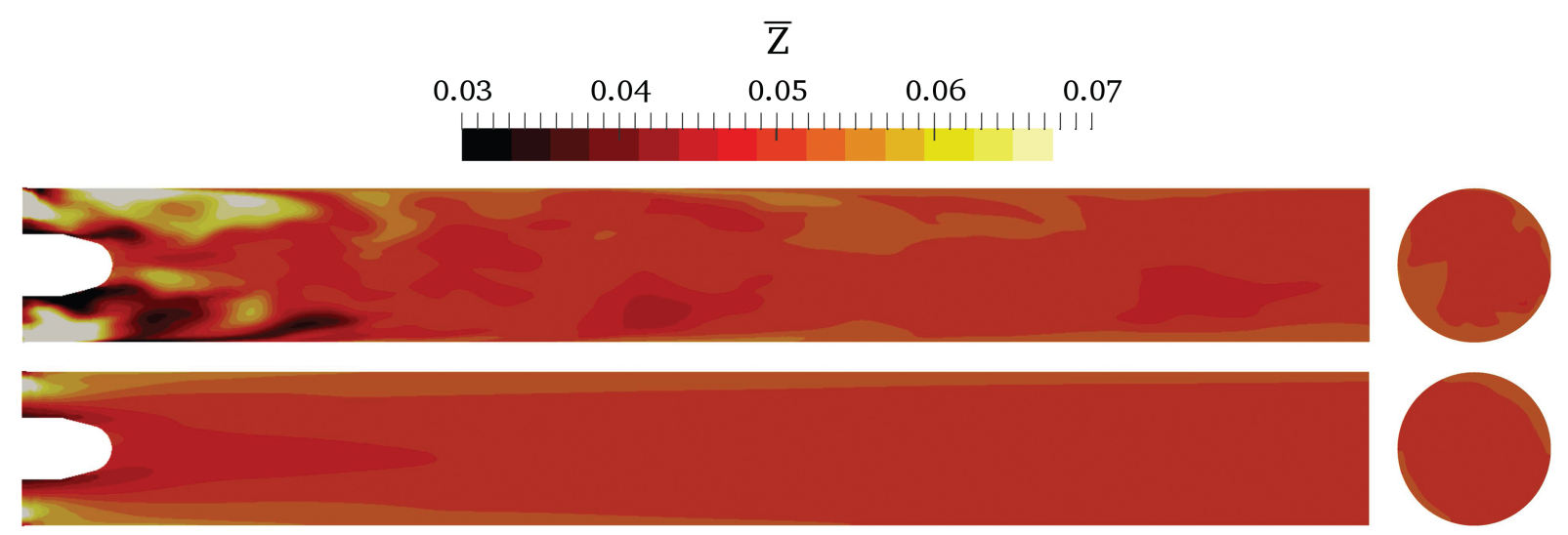

Figure 6.6: Instantaneous (top) and averaged (bottom) fuel mixture fraction of the Baseline case at a mid-plane within the premixing duct. The axial contours are located $50 \mathrm{~mm}$ upstream of the chamber baseplate.

The mean profile shows that the flow is decelerated after the the lance due to the 
area increase, but it accelerates further downstream due to the increase in the boundary layer thickness. The axial section shows that the mean velocity field is axisymmetric. Similarly, the mixture fraction distributions are plotted in Figure 6.6. The instantaneous image shows how rich pockets are formed and mixed with the leaner surrounding mixture, while the average field shows how the mixing level increases progressively with distance. At the inlet of the combustion chamber the fuel and air are technically premixed, being the mixture fraction slightly higher at the duct wall.

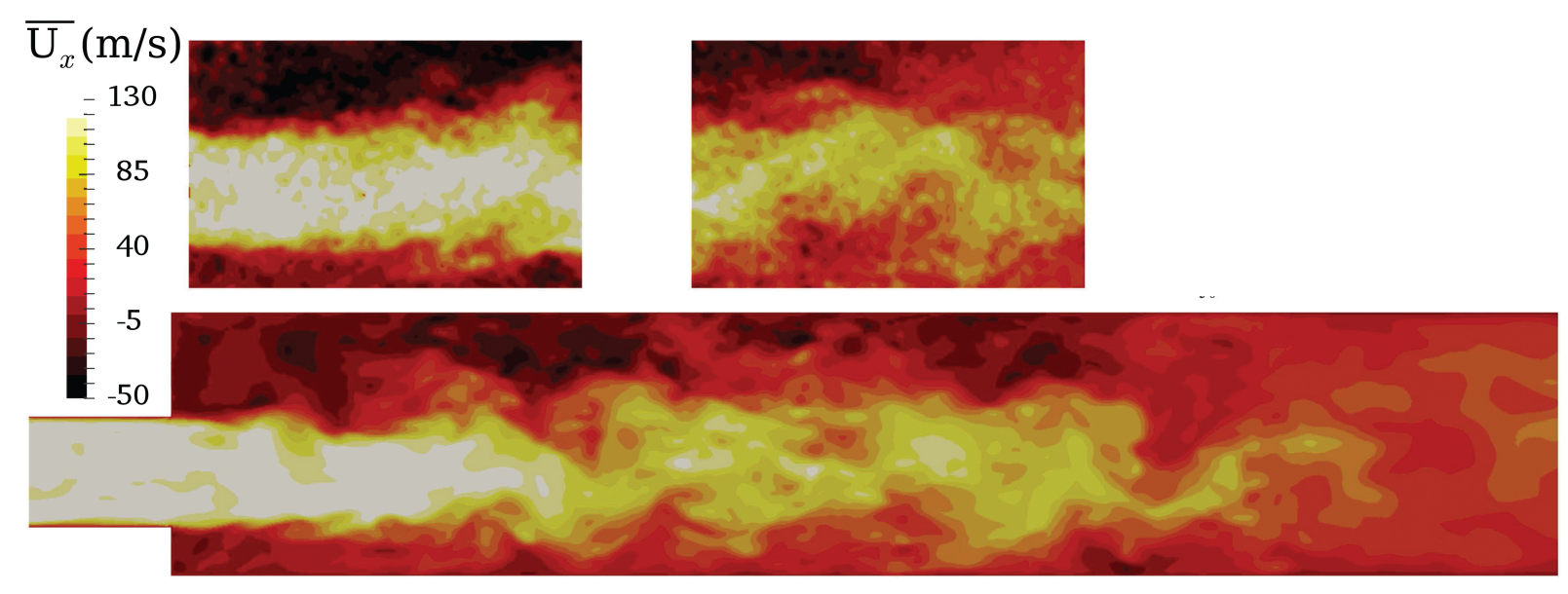

Figure 6.7: Instantaneous PIV data (top) and predicted axial velocity (bottom) of the Baseline case at a mid-plane within the combustion chamber.

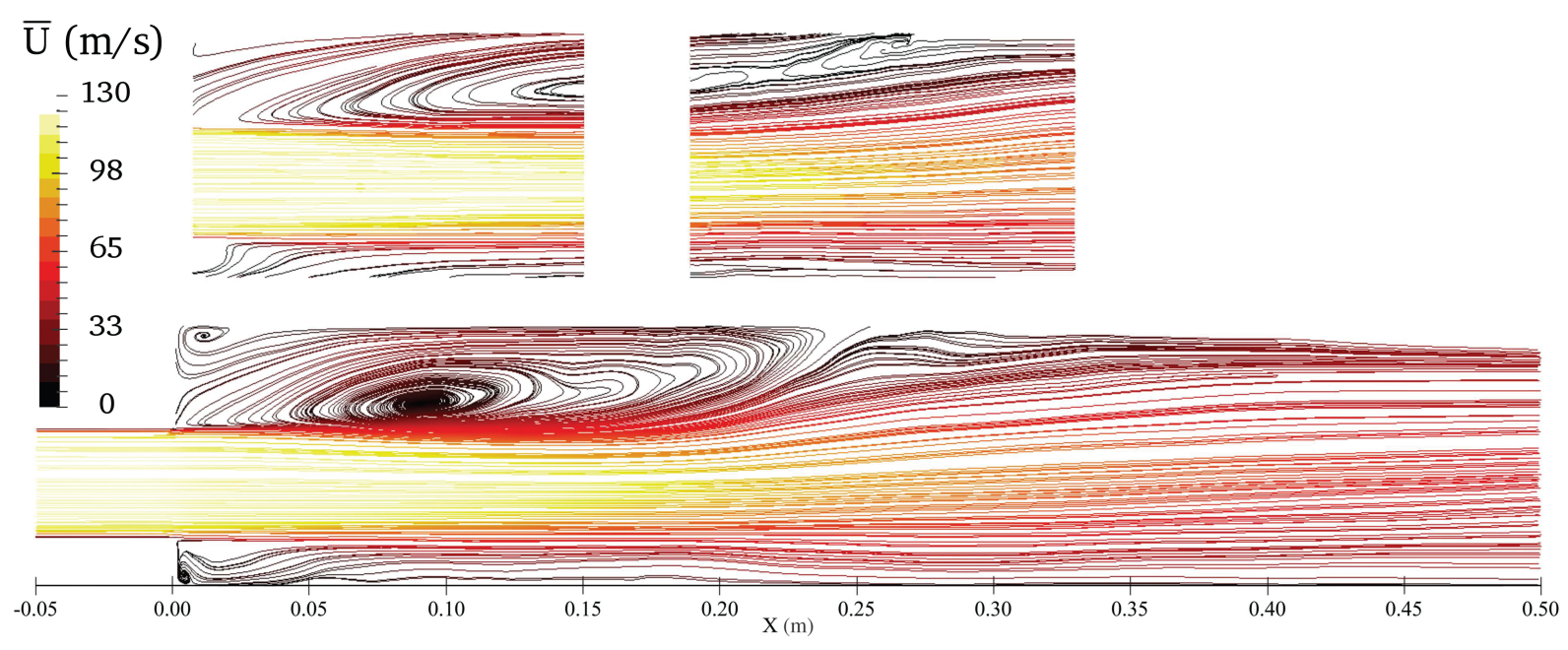

Figure 6.8: Time-averaged PIV (top) and CFD (bottom) streamlines of the Baseline case at a mid-plane within the combustion chamber.

Figure 6.7 compares instantaneous axial velocity contours from CFD and PIV at the chamber mid-plane, which show a highly turbulent flow within the jet and at the recirculation zones. No data is available at the middle of the PIV image because a strut is placed between the glass windows (see Figure 6.1). Larger turbulent structures are obtained in the simulation due to the spatial discretization. Despite two instantaneous results are being compared, both the initial jet velocity and the jet length are qualitatively 
similar. The latter is more evident in Figure 6.8, where time-averaged results are plotted by means of flow streamlines coloured with the mean velocity magnitude. Although the centre of the recirculation is located about $70 \mathrm{~mm}$ upstream of the location in the PIV data, the recirculation length is well predicted. On the bottom side the direction of the streamlines follow the same trend, from the wall towards the jet, as in experimental data.
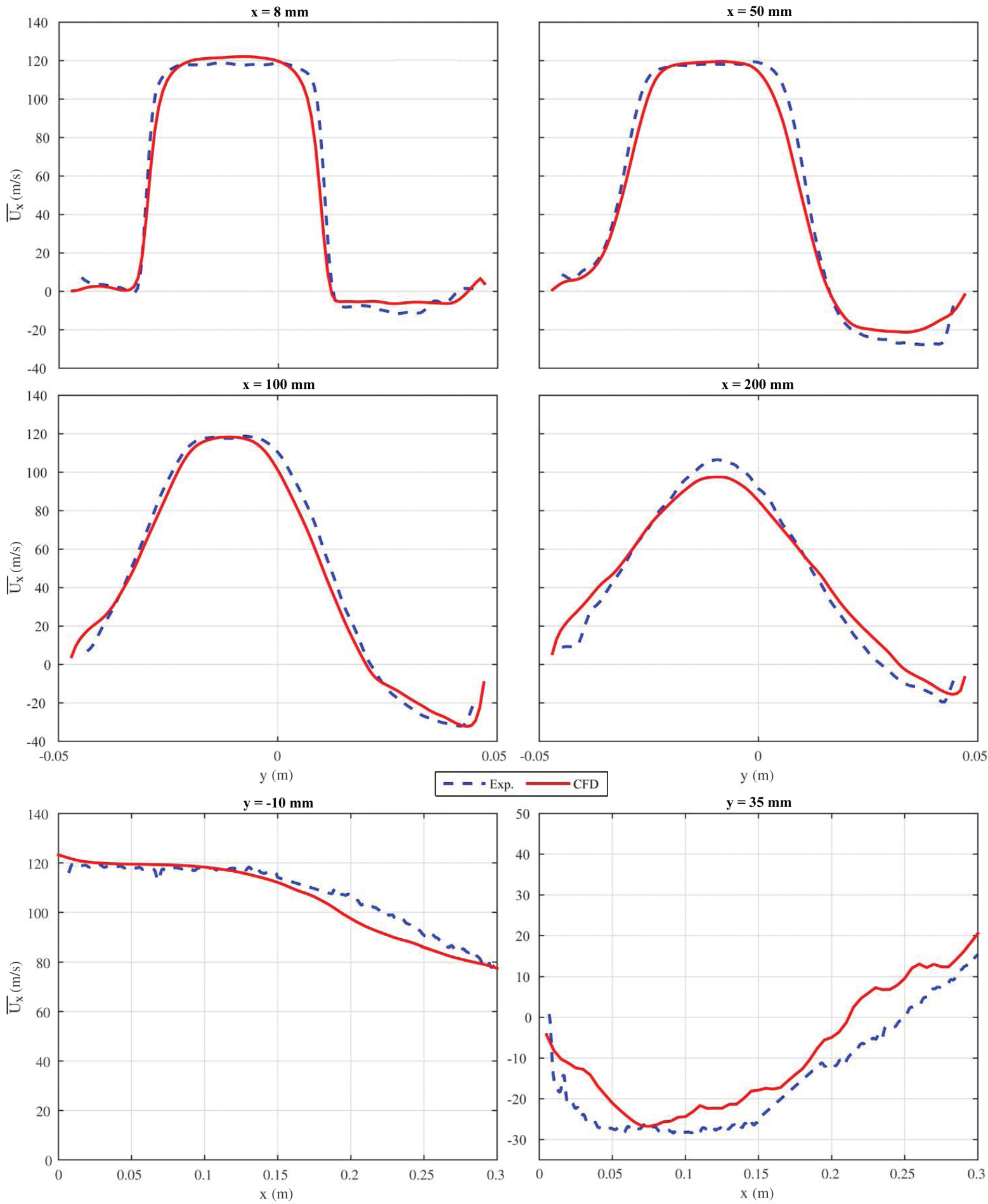

Figure 6.9: Comparison of the axial velocity profiles from Figure 6.8 at four axial lines, downstream of the baseplate, and two longitudinal lines at the jet center and at the middle of the large recirculation region. 
To have quantitative comparison, four axial lines and two longitudinal lines are extracted from the contours in Figure 6.8 and plotted into Figure 6.9. For the logitudinal lines, the PIV data is interpolated in the region where it is not available. In general, a good agreement with the experimental data is obtained. Despite a slightly steeper velocity gradient at $x=8 \mathrm{~mm}$ is observed in the measurements, a similar bulk velocity is obtained. The next two lines show that the simulation is able to predict the velocity spread due to the shear layers as well as the reverse flow velocity. From $x=100 \mathrm{~mm}$ to $x=200 \mathrm{~mm}$ the decay of the velocity at the jet centre due to the higher effective area is over-predicted by $10 \mathrm{~m} / \mathrm{s}$, so that a higher velocity is obtained at the sides. The decay of the velocity along the jet axis corresponds to the plot for $y=-10 \mathrm{~mm}$. The line at $y=35 \mathrm{~mm}$ shows the axial velocity across the top recirculation region, which is over-predicted at some regions by around $15 \mathrm{~m} / \mathrm{s}$ while the main trend is followed.

The tabulated chemistry model does not calculate the concentration of the $\mathrm{OH}$ radicals within the flame. In order to compare simulation results with the chemiluminescence data, the progress variable source term (which can be related to the flame heat release) is employed. To verify this assumption, a 1-D flame at the same premixed conditions as in the Baseline case is computed with Cantera and profiles for $\mathrm{X}_{\mathrm{OH}}$ and $\dot{\omega}_{\mathrm{CO}_{2}}$ are plotted into Figure 6.10. Although the $\mathrm{CO}_{2}$ source term profile is thinner and falls down to zero after the flame, the same peak position is obtained for both variables. Thus, this assumption is found to be adequate for a qualitative comparison and has also been verified for the oil flames.

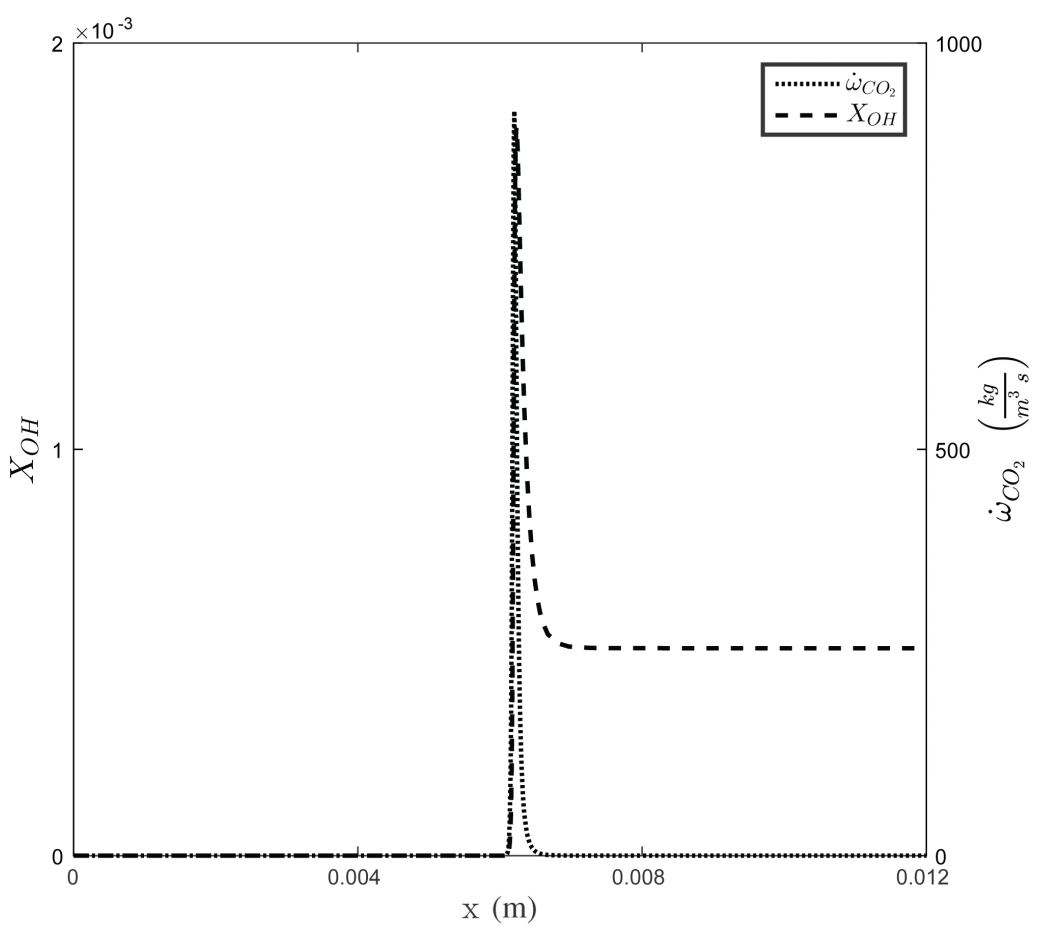

Figure 6.10: Profiles for the progress variable source term and $\mathrm{OH}$ molar fraction from a detailed solution using the GRI3.0 mechanism at similar conditions as the Baseline case. 
The time-averaged depth-integrated progress variable source term is compared to the $\mathrm{OH}$-chemiluminescence image in Figure 6.11 for the cases operated with gas fuel. Although both contours can only be qualitatively compared, in terms of flame position and intensity change between the cases, a significant flame lift at the lower side of the chamber is observed in the experiments. This effect is well-predicted in the simulation and it is attributed to the heat losses at the chamber walls, modelled by imposing a wall temperature distribution, as well as to the flame-turbulence interaction. Simulations with adiabatic chamber walls or with a doubled flame-turbulence interaction coefficient present a flame that is attached to the baseplate. Apart from this, a similar flame length is also obtained. Furthermore, the intensity increment from the Baseline to the Propane cases is also in good agreement with the experimental data.
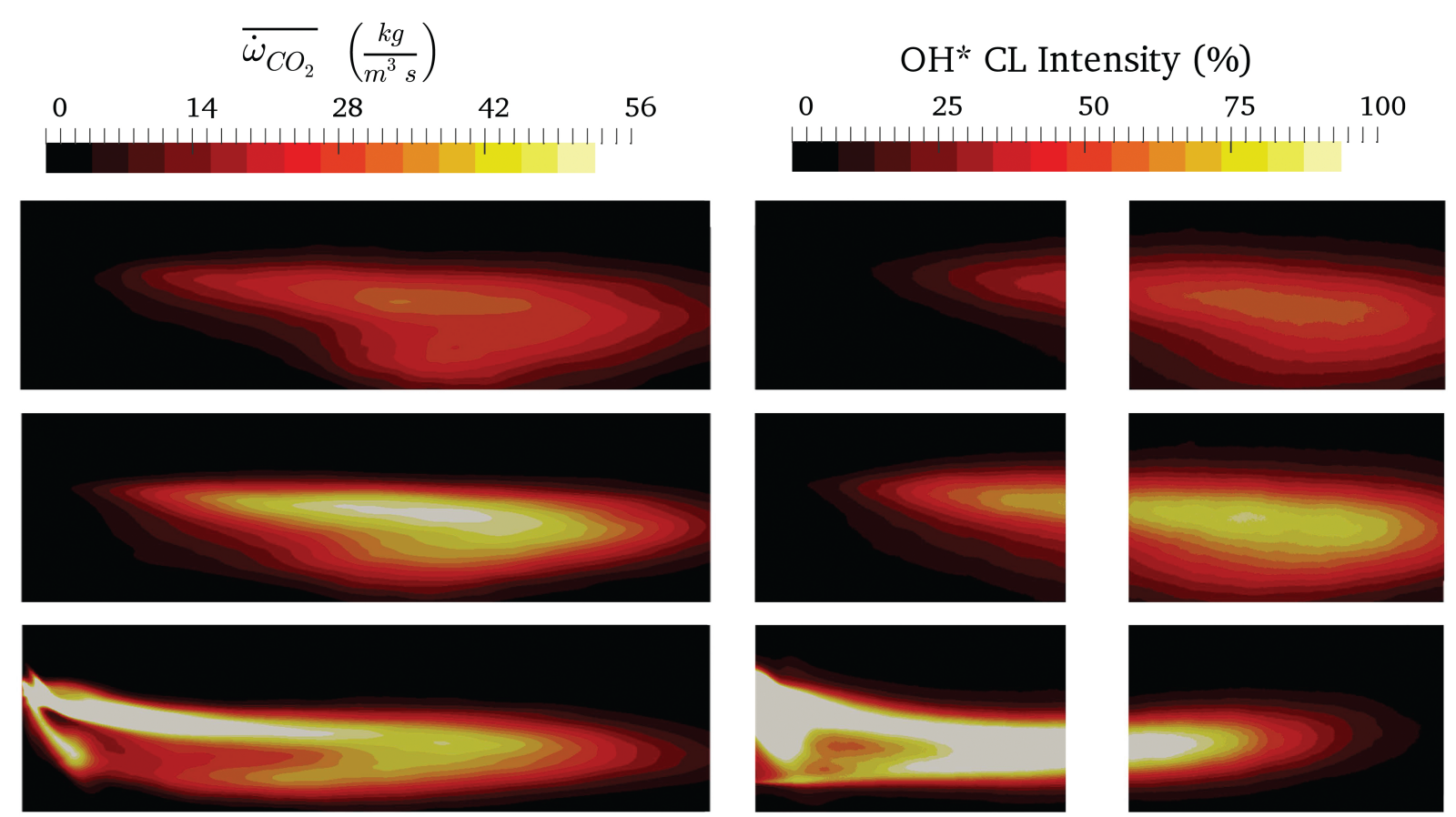

Figure 6.11: Chemiluminescence data (right) and width-integrated progress variable source term (left) for the Baseline (top), Propane (middle) and Piloted (bottom) cases.

Both the instantaneous and average temperature profiles at the chamber inlet and mid-plane are included in Figure 6.12. As the fuel is well mixed with the air, there are no significant temperature differences in the axial profile. The instantaneous contour shows the wrinkling of the flame due to the turbulence interaction, which potentially promotes the formation of pockets. The averaged plot displays a progressive temperature increase along the jet axis until a maximum value is achieved. The downstream temperature falls mainly because of the temperature imposed at the chamber walls, but also due to the mixing. Since the progress variable source term, presented in Figure 6.11, is associated to the heat release, a higher flame temperature is obtained for the Propane simulation. 


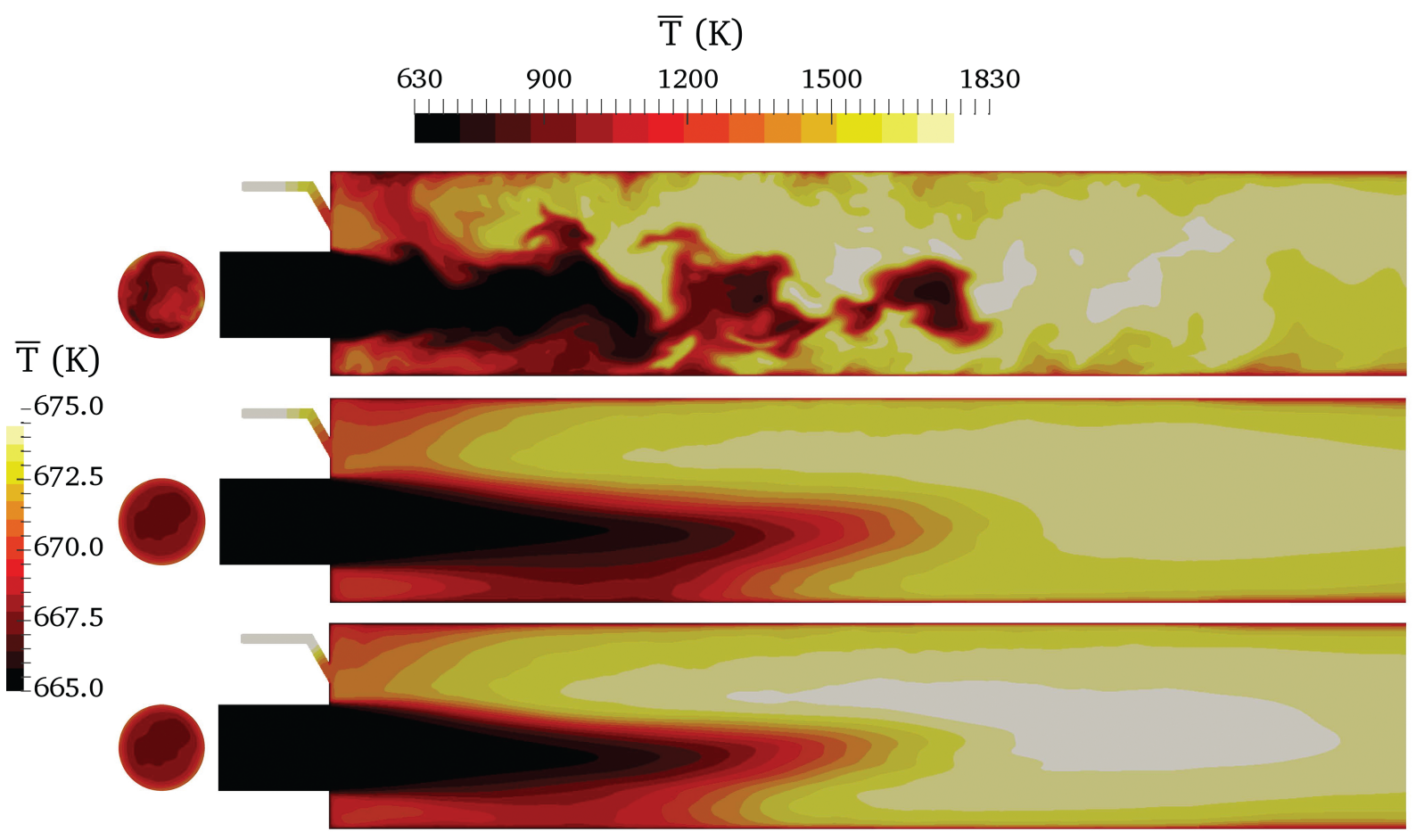

Figure 6.12: Instantaneous (top) and averaged (middle and bottom) temperature fields of the Baseline (top and middle) and Propane (bottom) cases at a mid-plane within the combustion chamber. The axial contours are located $50 \mathrm{~mm}$ upstream of the chamber baseplate.

\section{Piloted case results}

For the Piloted case, since the conditions upstream of the chamber are the same as in the Baseline case, only the fields at the chamber are presented.

In Figure 6.13, the velocity contour shows that the middle pilot jet is deflected and merges with the main jet, which in turn decelerates due to the momentum exchanged. The upper recirculation zone is oval-shaped and bigger than in the Baseline case. The middle contour illustrates how the stoichiometric mixture introduced through the pilot tubes is mixed with the main jet, leading to a higher local equivalence ratio. The greater concentration at the bottom region originates from the outer pilot jets, as there is less interaction with the main jet and therefore they penetrate further. Regarding the temperature distribution within the chamber, the maximum temperature originates right downstream of the pilot flames. Compared to the results for Baseline case in Figure 6.12, the influence of the pilot also increases the main flame temperature. As with the mixture fraction, a high temperature region at the bottom side can be also identified.

The quantitative comparison of the axial velocity with experimental PIV data is also performed for the Piloted case in Figure 6.14. Higher discrepancies than in Figure 6.9 are observed. At $x=8 \mathrm{~mm}$ the main jet bulk velocity is over-predicted by around $10 \mathrm{~m} / \mathrm{s}$. The simulation shows that, at this position, the profile is already affected by the central pilot jet since a second peak appears. It is not well known if this peak, which is not 


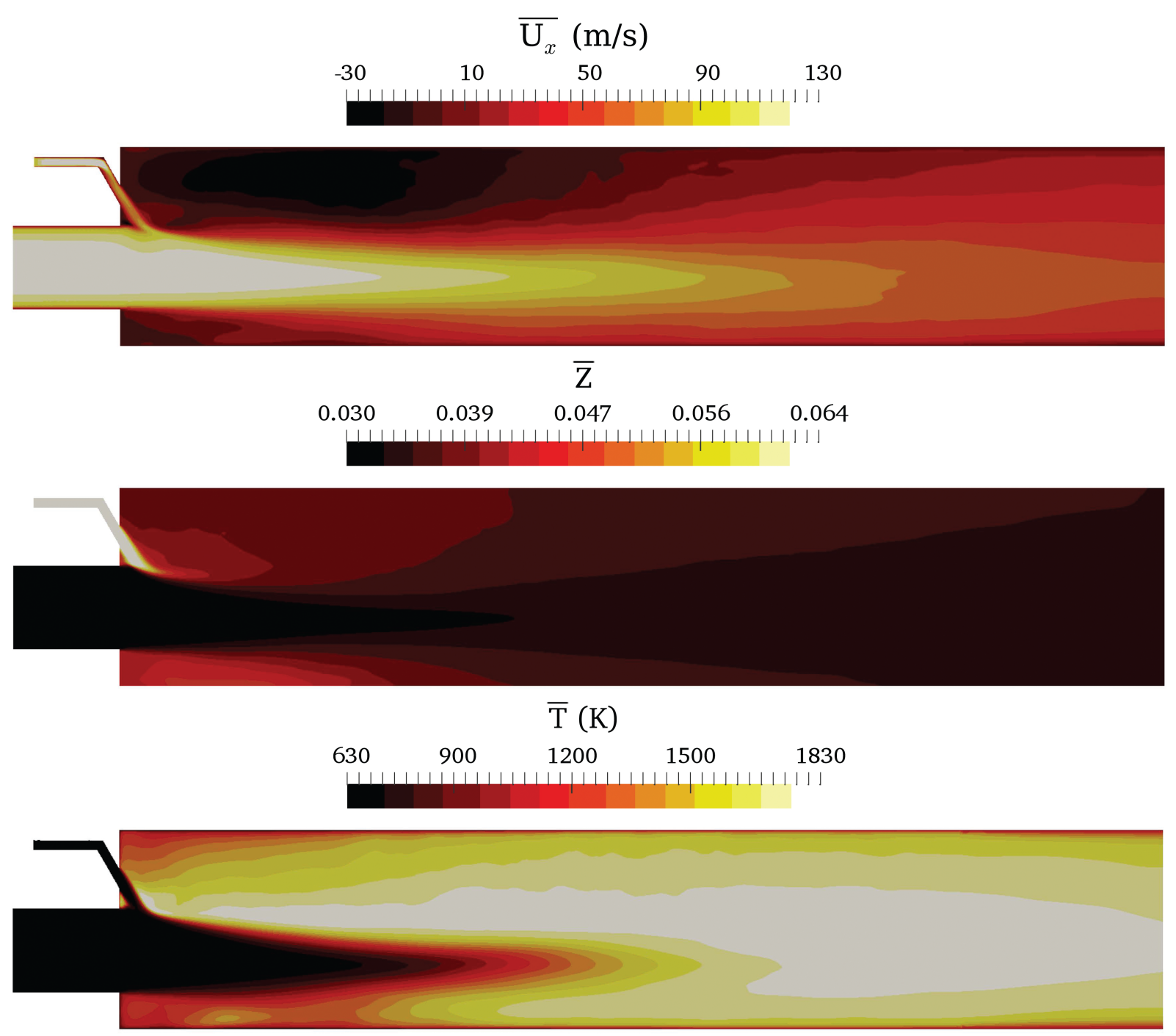

Figure 6.13: Time-averaged axial velocity (top), mixture fraction (middle) and temperature (bottom) distributions at the front of the chamber for the Piloted case.

visible in the measurements, is not physical or whether the PIV misses it because a low amount of particles are present at that region. Despite the higher jet velocity, a similar velocity spread is obtained at $x=50$ and $100 \mathrm{~mm}$. A good agreement is also found for the longitudinal lines. At the last axial line, however, differences become more significant. The centre of the jet in the simulations is not aligned with the one in the PIV data and a significant velocity over-prediction, of about $30 \mathrm{~m} / \mathrm{s}$, is observed at the left side. The latter suggests that the flame shape is not completely well captured. This can be observed in the flame source term comparison with the OHCL image in Figure 6.10.

Two shapes of the pilot flames are identified depending on whether the main jet affects them or not. For the outer tubes, the flame has the same direction as the pilot jet entering the chamber. In the simulations, these flames are more concentrated than in the experiments and slightly longer. For the inner tubes, where the pilot flame is deflected by the main flow, a similar shape as in the OHCL contour is observed. The main flame 
is slightly shorter and more intense than in the Baseline case, due to the influence of the pilot flames, but it is wider and less intense than in the test data.
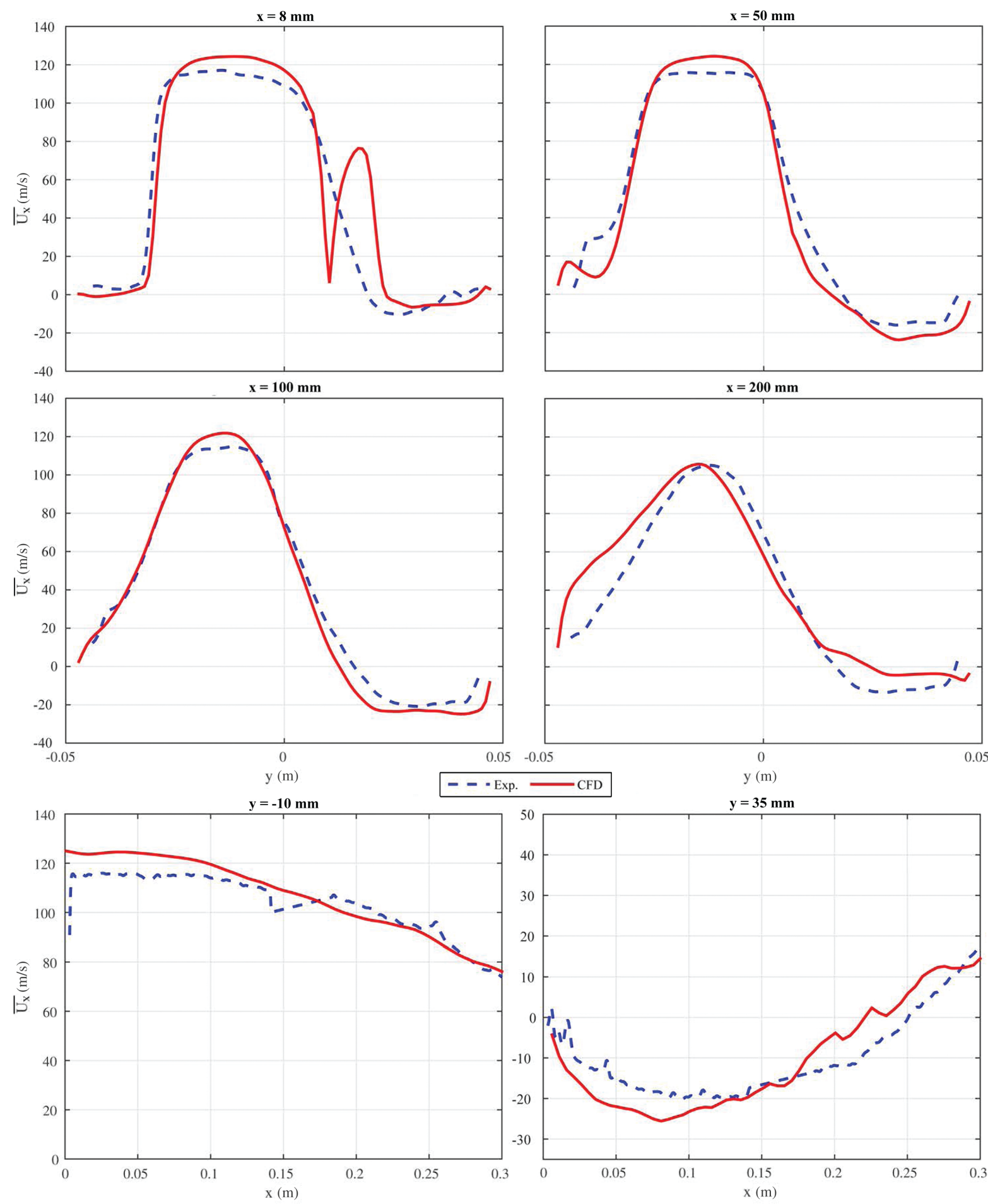

Figure 6.14: Comparison of the axial velocity between PIV data and CFD results for the Piloted case at four axial lines, downstream of the baseplate, and two longitudinal lines at the jet center and at the middle of the large recirculation region.

Additionally, in the simulation a lifted flame can be observed at the lower side while in the experiment it is attached to the baseplate. The same effect can be observed at the 
root of the pilot flames. This indicates that the thermal boundary condition imposed at both the baseplate and the chamber walls is not appropriate, as the pilot flames should increase their temperature. Thus, results for the piloted case could potentially improve if a higher temperature distribution (i.e. lower heat losses) is imposed at the baseplate and the chamber walls.

To better understand the effect of the pilot tubes, temperature contours for both Baseline and Piloted simulations at several axial locations are compared in Figure 6.15. In the unpiloted simulation, despite the asymmetry, the temperature over the jet (dark circle) rises progressively with distance. At $x=100 \mathrm{~mm}$ the jet is slightly deformed due to the heat released over it. In the last plane there is still some fuel reacting but the high temperature region has extended around the jet, being the maximum value located on the upper side of the chamber.

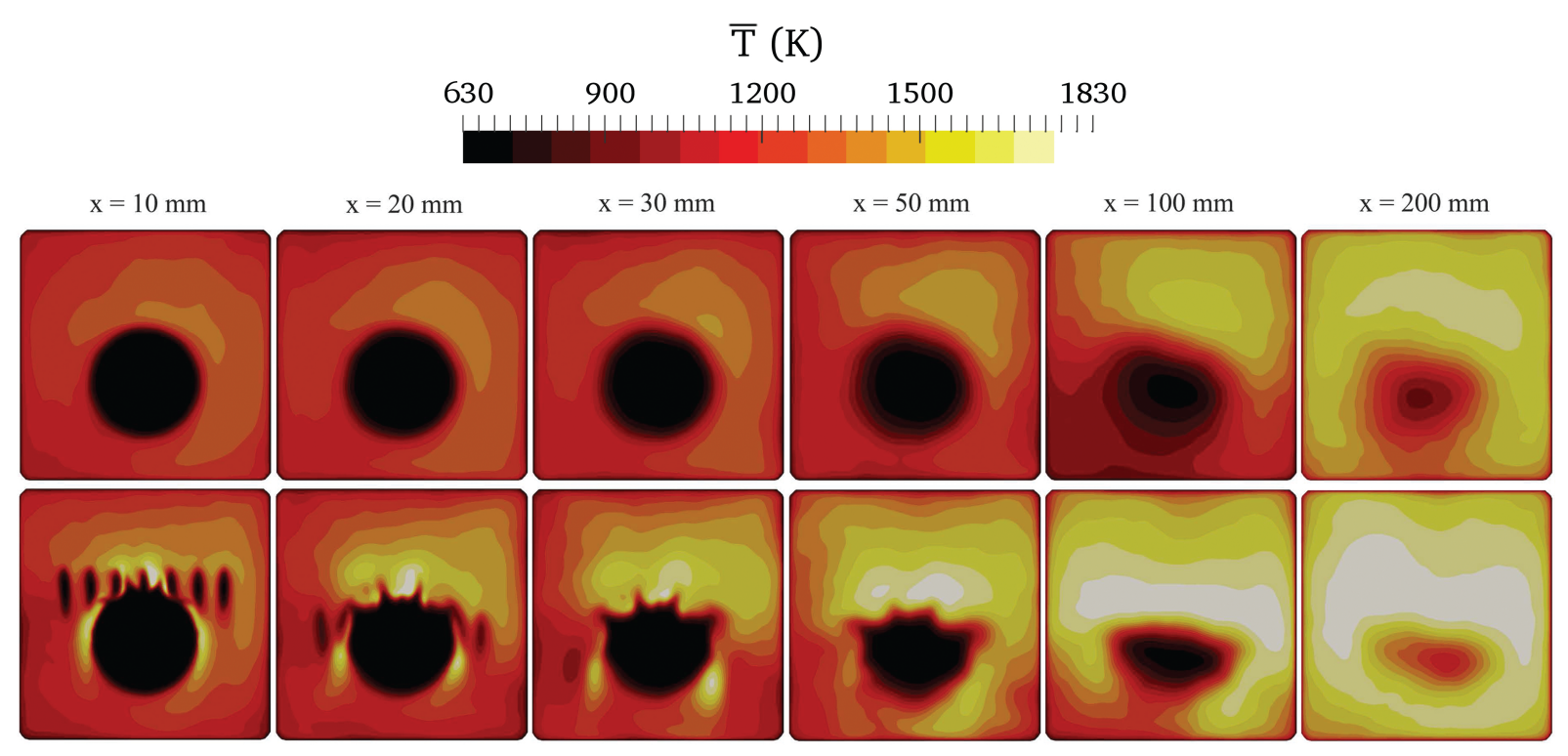

Figure 6.15: Averaged temperature fields of the Baseline (top) and Piloted (bottom) cases at several axial planes from the baseplate.

In the piloted contours, the seven jets can be identified at the first axial location. As observed in Figure 6.14, the central pilot jet has already interacted with the main jet by this position. High temperature regions, caused by the three central pilot frames are found both on top of the pilot jets and on the sides of the main jet. The next planes reveal that the outer pilot jets are transported downside and mixed to react further downstream. The deformation of the main jet starts at $x=20 \mathrm{~mm}$ and becomes significant by $x=100 \mathrm{~mm}$. The last plane shows that there is less fuel remaining than in the Baseline case and the high temperature regions are located also at the sides and below the main jet. 


\subsubsection{Liquid fuel}

In this section, results for the liquid fuel cases are presented. Due to the additional complexity, the validation for gas fuel performed in the previous subsection was necessary to ensure that the combustion model gives reasonable results. In this way, any discrepancy with the experiments should not be attributed to the combustion model itself but to either the spray models, the kinetic mechanism or the thermal boundary conditions imposed.

\section{Diesel}

Figure 6.16 displays the time-averaged spray statistics at three axial planes along the premixing duct. The liquid mass flow distribution is shown first, normalized with the overall injected mass flow. Although 8 independent jets are injected, the spray dispersion caused by the resolved turbulence leads to a combined cloud of droplets concentrated at the center. At the second plane, the liquid mass flow is significantly lower due to the evaporation process and it covers the complete circular section. The last plane, which corresponds to the inlet of the combustion chamber, shows that the spray is not completely vaporized in the premixing duct and a small amount of liquid enters into the combustion chamber.

Regarding the droplet temperature, a constant distribution is obtained at the first plane. At the second plane, however, the droplet temperature is higher at the duct walls than at the centre. The latter is caused by the spray interaction with the boundary layer at the wall. The droplets at the centre heat up further so that a roughly uniform temperature is found at $x^{\prime}=380 \mathrm{~mm}$. For the size distribution, the atomization model results in a mean droplet size of about $46 \mu \mathrm{m}$. As the spray progresses, the SMD decreases at the walls while spots with higher SMD appear because of the local evaporation of the small droplets. Regarding the spray velocity distribution, the highest values at the $x^{\prime}=55 \mathrm{~mm}$ plane are found where there is no significant liquid mass flow (i.e. low inertia) while the lowest are are located at the centre. The decrease in the gas phase velocity due to the momentum exchange with the spray (2-way coupling) contributes to this profile. As the spray is transported downstream and gets further vaporized, it accelerated to reach the gas phase velocity. The evolution of these variables with the distance from injection is further analysed in Section 6.4.2.

The gas phase velocity, mixture fraction and temperature fields within the premixing duct are presented in Figure 6.17. Although a similar velocity profile as in the Baseline case is obtained, the velocity at the duct exit is lower. This is caused by the increased momentum required to accelerate the fuel and a greater temperature drop due to fuel evaporation and mixing. The mixture fraction contour shows that the fuel is not fully premixed with the air at the entrance of the chamber, but the concentration is larger at the duct wall and therefore closer to stoichiometric conditions $\left(Z_{s t}=0.063\right)$. The 

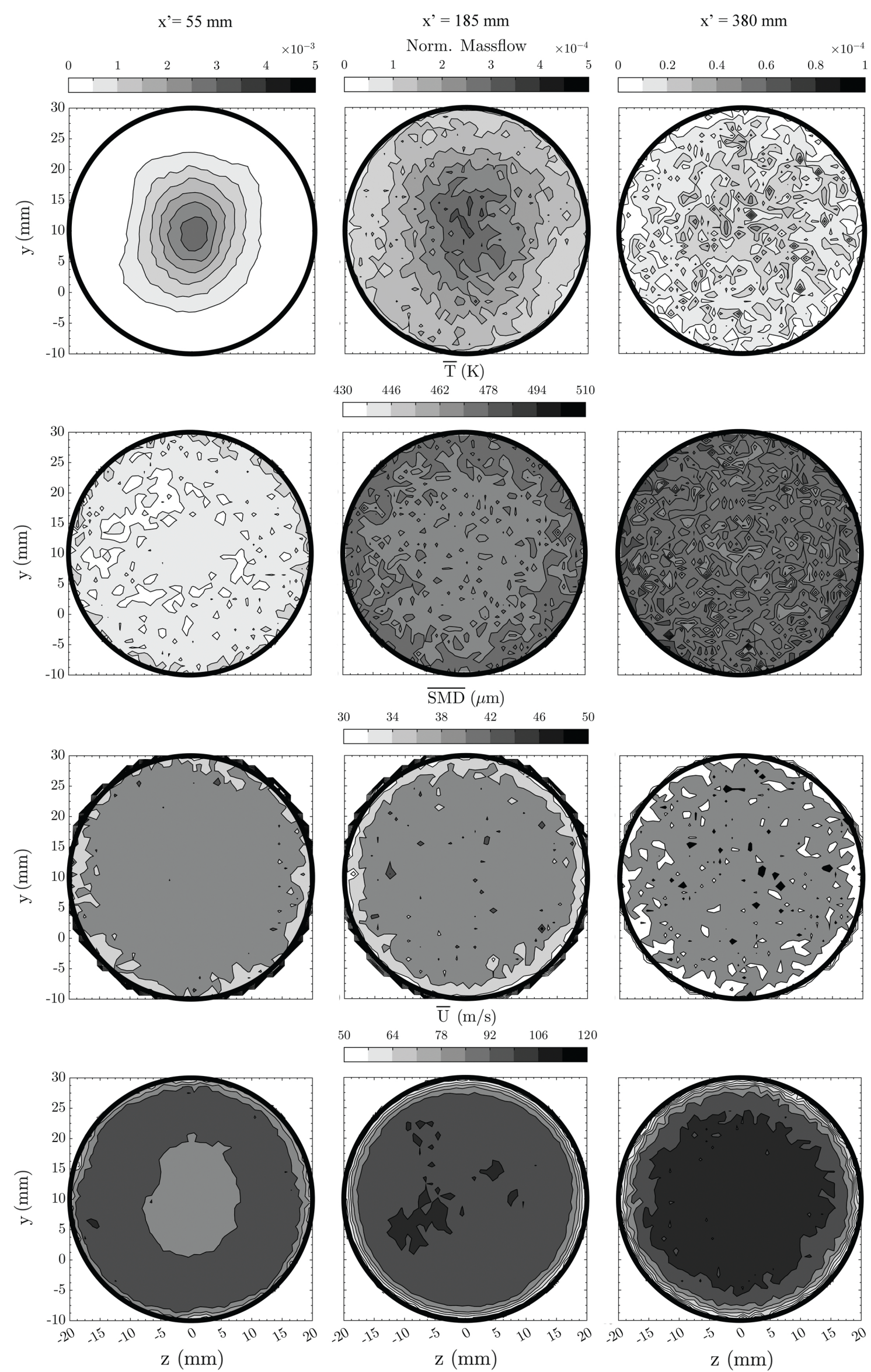

Figure 6.16: Time-averaged spray statistics at three axial planes placed along the premixing duct for the Diesel case. 
inhomogeneity in both temperature and mixture fraction fields justifies the range of the variables employed for the tables generation in Section 6.3.1. Although rectangular tables were created, most of the 1-D flame solutions are not used because low temperature regions coincide with high fuel concentration and vice versa. Therefore, tables can be optimized in future work to have a higher resolution at the relevant conditions while keeping a reasonable size.

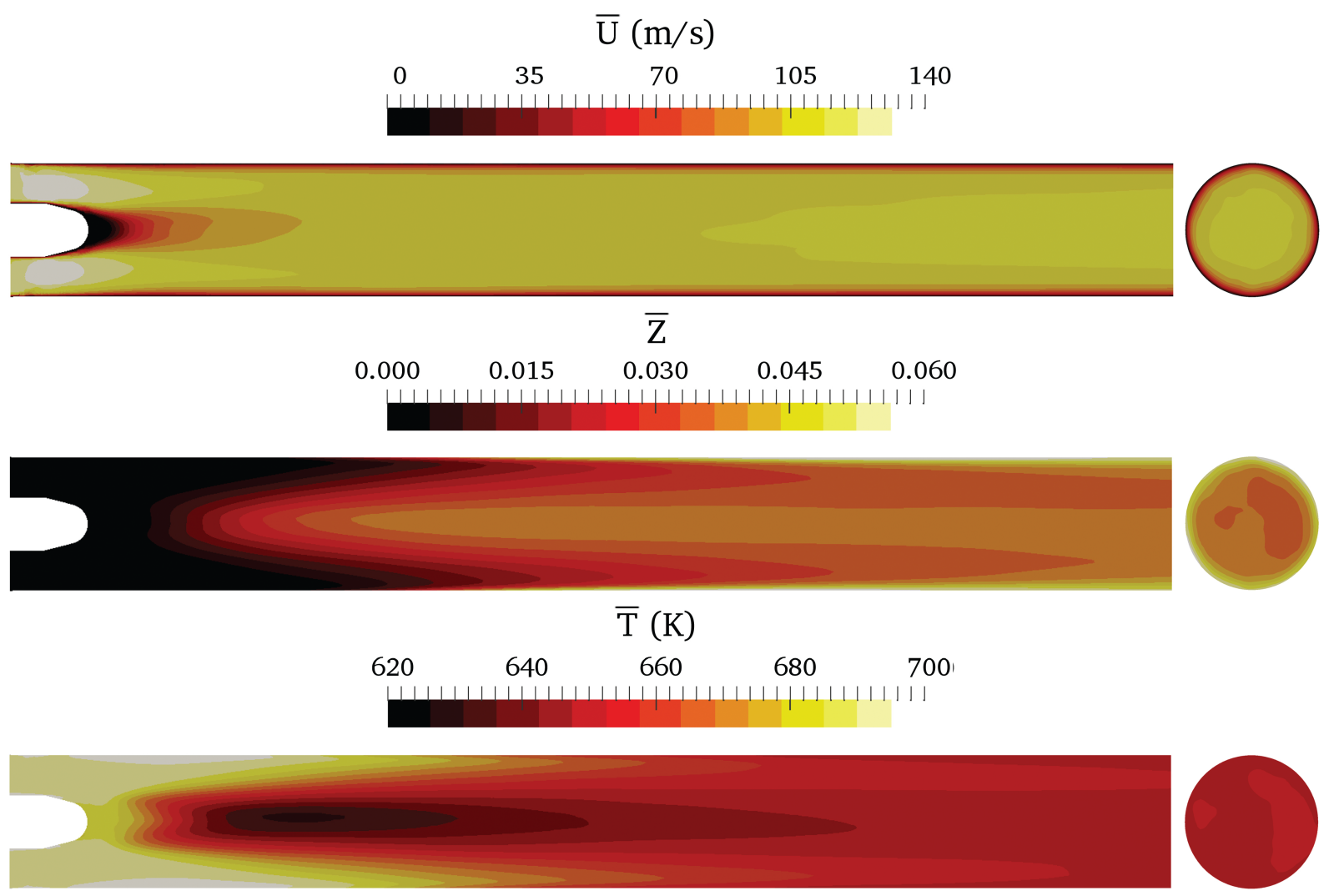

Figure 6.17: Time-averaged gas phase axial velocity (top), mixture fraction (middle) and temperature (bottom) distributions at the mid-plane within the premixing duct for the Diesel case. The axial contours are located $50 \mathrm{~mm}$ upstream of the chamber baseplate.

The mixture fraction and temperature distributions at the mid-plane of the combustion chamber are illustrated in Figure 6.18. As the mixture enters, it is further mixed due to the turbulence induced by the jet so that the equilibrium mixture fraction is almost reached at $x=500 \mathrm{~mm}$ (right side of the contour). Most of the remaining droplets are completely vaporized when they reach the flame front, although few of them are large enough to cross it and travel a few centimetres. The evaporation of these droplets increases the mixture fraction and consequently the equilibrium of the $\mathrm{CO}_{2}$ species, so that the progress variable source term rises. The high fuel concentration at the premixing duct walls induces a high temperature region at both sides of the jet. Clear differences are found in comparison to the gas fuel case in Figure 6.12, where the maximum temperature is about $200 \mathrm{~K}$ lower and it is located at the flame tip. A more detailed comparison of the flame shape and intensity between other cases and experimental data is performed in the next section. 


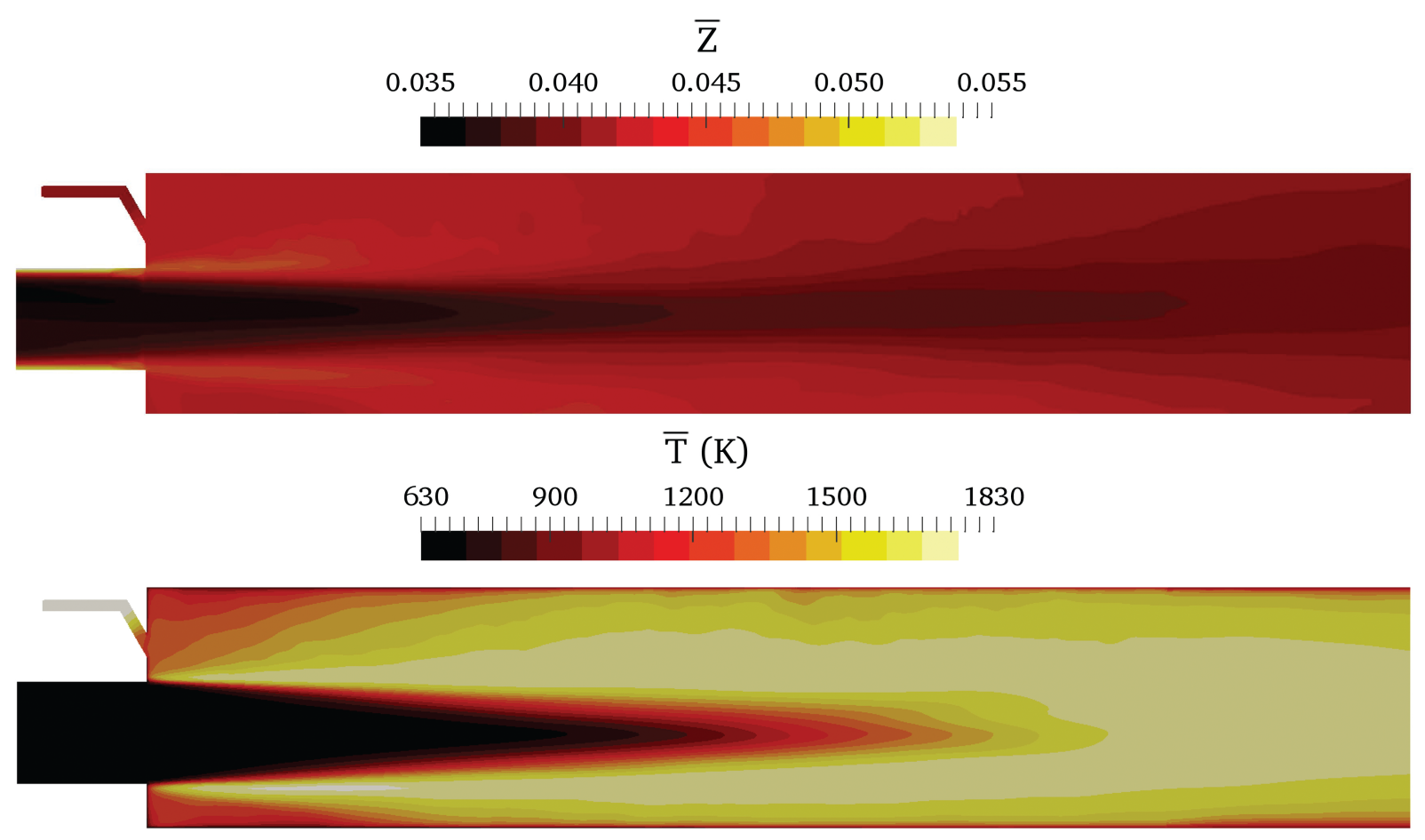

Figure 6.18: Time-averaged mixture fraction (top) and temperature (bottom) distributions at a mid-plane within the combustion chamber for the Diesel case.

\section{Emulsion and Preheated}

The normalized liquid mass flow distribution is plotted for the cases with water addition at the same axial positions as in Figure 6.16.

In both cases the liquid-air momentum ratio is the same, but higher than for the dry oil case, to achieve a similar penetration. Consequently, the global equivalence ratio of the Preheated case is about $10 \%$ larger than the Emulsion one. A greater mass flow is obtained for the Emulsion case at each plane, due to the lower inlet temperature, while the fuel distribution pattern is similar. Compared to the dry oil case at the first axial plane, the sprays are also concentrated at the center, but the area covered is larger. At the second plane the fuel is not concentrated at the channel center any more and high mass flow regions appear near the wall. The rear plane shows a faster spray vaporization in the central region than at the near-wall.

The spray characteristics along the premixing duct for the Diesel, Emulsion and Preheated cases are compared in Figure 6.20. This is done by means of global values obtained from the area-average of the relevant fields at six planes separated by $55 \mathrm{~mm}$, three of which correspond to the planes used in Figures 6.16 and 6.19.

The mass flow graph shows that, despite the higher spray temperature, the evaporation rate decreases at the rear part of the duct $(x>190 \mathrm{~mm})$ driven by the higher vaporized fuel concentration at the droplets' surface. An increased heat transfer takes place between the second and third planes, as the slope from the first to the second plane is maintained 

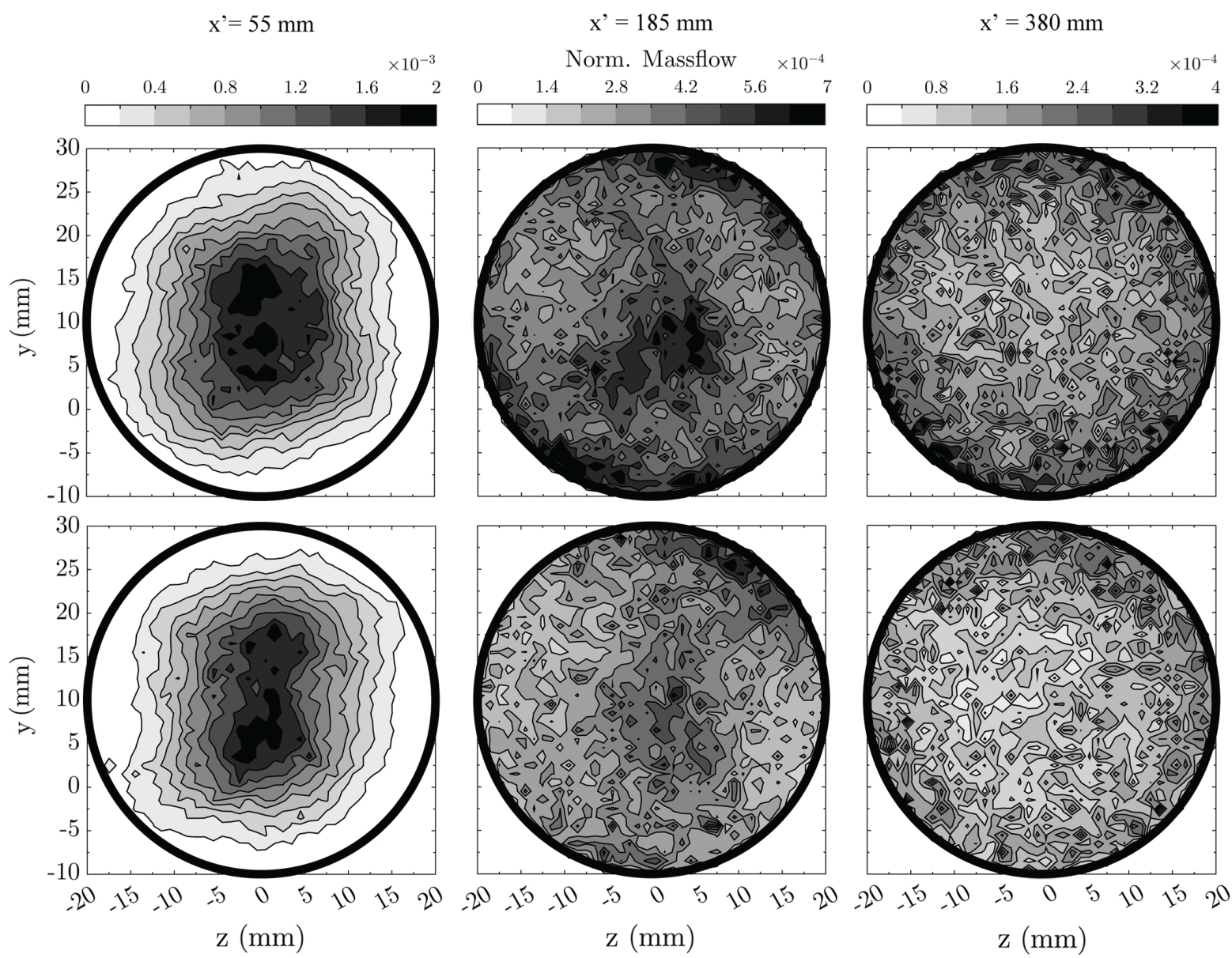

Figure 6.19: Time-averaged normalized spray mass flow at three axial planes placed along the premixing duct for the Emulsion (top) and Preheated (bottom) cases.

in the dry oil case and it even increases for the wet oil cases. This is caused by the increase in the relative velocity due to wall interaction, since the spray velocity shows a significant decay between these planes. While about $3 \%$ of the injected mass flow remains at the inlet of the chamber for the dry oil case, about $27 \%$ and $20 \%$ results from the Emulsion and Preheated cases respectively. The main reason for this is that the ratio between the injected and the air mass flows for the latter cases is around $80 \%$ higher than the ratio for the Diesel case. Additionally, water requires more energy to heat up and vaporize due to the larger specific heat capacity and latent heat. This also limits the evaporation of oil due to the lower droplet temperature. The evolution of the water mass flow has also been plotted, in relation to the injected water mass flow. It shows that near the injection mostly water is vaporized and from plane at $x^{\prime}=180 \mathrm{~mm}$ on, the evaporation of oil becomes significant. By the end of the premixing duct, around $7 \%$ of the injected water is still liquid for the Emulsion case and 3\% remains for the Preheated case.

The initial SMD values for the dry oil case are larger because the addition of water reduces the liquid viscosity and therefore the stripped droplets from the liquid jets are smaller. As the spray progresses, after an initial decrease, the SMD increases at 

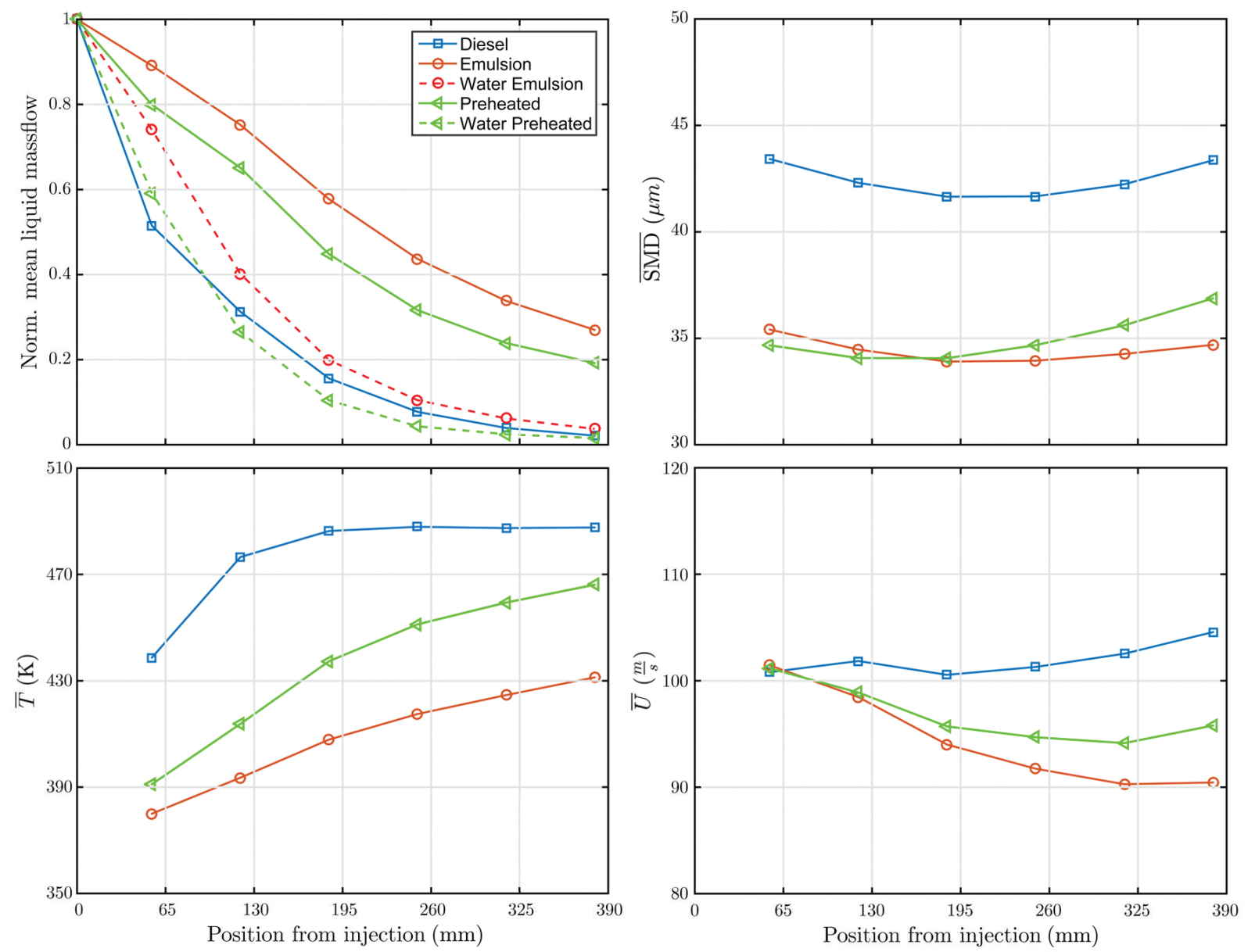

Figure 6.20: Evolution of the global time-averaged spray mass flow (top left), SMD (top right), temperature (bottom left) and velocity (bottom right) along the mixing duct for the cases employing liquid fuels.

the rear planes because the small droplets are completely vaporized. This effect is more pronounced in the Preheated case, but can also be observed in the other liquid fuel simulations.

Regarding the spray temperature, a rapid increase is observed for the dry oil case along the first $120 \mathrm{~mm}$ from the injection and it remains constant further downstream. A significantly slower temperature increment is found for both cases with water addition, especially for the Emulsion case. As water gets vaporized, the temperature of the droplets approaches the equilibrium temperature obtained in the Diesel case. Since the superheat limit temperature of water is not achieved, the micro-explosion phenomena is very unlikely to occur within the premixing duct for the investigated cases.

The velocity profiles present a different behaviour between the dry and wet cases. While the spray velocity mostly increases with distance in the Diesel case, it decreases in the other cases because part of the liquid starts to interact with the channel wall before the second plane. This trend starts to change at the rear side of the channel, due to momentum transfer from the gas phase, so that a slight velocity increase is obtained 

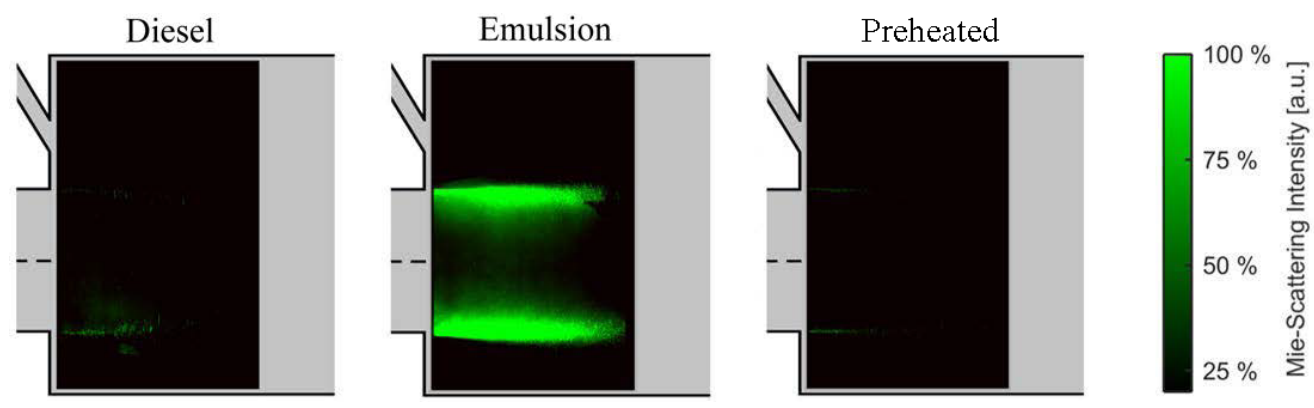

Figure 6.21: Mie scattering measurements at the front side of the combustion chamber.

at the last plane in the Preheated case. Thus, droplet-wall interactions become more important for the wet cases because of the higher momentum ratio.

Time-averaged Mie scattering measurements at the mid-plane of the chamber are depicted in Figure 6.21, which qualitatively show the actual amount of liquid that enters into the combustion chamber. Few particles are observed for the dry oil case, particularly at the duct wall. This is in agreement with the small liquid mass flow profile obtained in Figure 6.20. When water is added to the fuel, a significant amount of liquid particles comes out from the duct, especially at the walls. With setting conditions of the Preheated case, it can be observed that the liquid intensity is similar to the one measured for the dry oil case.

Since the Preheated simulation predicts a mass flow about 15 times larger than in the Diesel case, a significant discrepancy is found. There are several aspects that can cause or contribute to this mass flow over-prediction:

- The atomization model over-predicts the droplet diameters for an emulsion jet in cross flow and this error is amplified by injecting 8 jets.

- The spray dispersion is too low. This might be caused by a low level of resolved turbulence, a wrong air inlet turbulence level (no turbulence imposed) or the need to additionally combine the atomization model with a sub-grid dispersion model.

- The distribution of water during the atomization process may not be properly modelled. For example, if the stripped droplets are assumed to be entirely composed of oil (continuous phase in the emulsion), water would be concentrated within the jet and the primary droplets. This would result in droplets with high water content and droplets without water, which could result in a faster evaporation of the spray.

- The use of DF2 as a surrogate of the diesel fuel used in the experiments, instead of using a more appropriate multicomponent fuel discretization, has an impact in the evaporation time.

- The shell evaporation model for emulsified droplets may not be appropriate, specially after the oil layer is depleted. A small under-prediction in the evaporation 
rate can have a significant impact in the complete spray evaporation due to the large amount of droplets in the domain. Despite reasonable results are obtained for the dry oil case, uncertainties in the evaporation model may become more evident with higher liquid-air mass flow ratios.

- The assumption of droplet rebound when reaching a wall boundary is inappropriate. Droplets can also break up or attach to the wall and form an evaporating film that is convected downstream at a slower velocity.

- Radiation from the flame can affect the spray evaporation rate and it should not be neglected in the simulation of reacting sprays.

Although some statements are more reasonable than others, further studies and experimental data are required to determine the error sources. For completeness, the main gaseous phase fields are analysed for both wet oil cases.

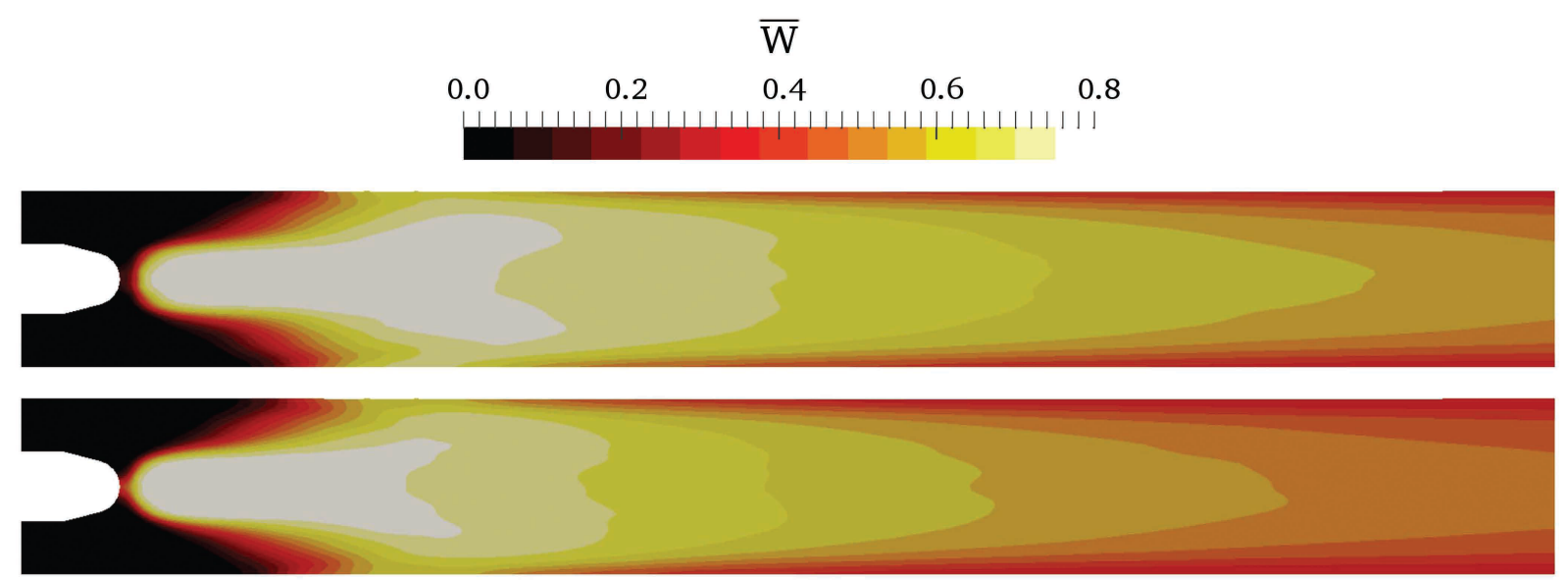

Figure 6.22: Time-averaged gas phase fuel water content at a mid-plane within the premixing duct for the Emulsion (top) and Preheated (bottom) cases.

Figure 6.22 corresponds to the water content distribution at the premixing duct. The highest water content is located near the injection points because water is more volatile than DF2. As the spray temperature increases and the DF2 evaporation becomes significant, the water content decreases progressively. The center line shows a linear decrease in water content at the first half of the duct. Similarly to the liquid mass flow curve in Figure 6.20, this reduction becomes slower at the rear side of the duct with a lower evaporation rate.

The shape of the temperature and mixture fraction fields within the premixing duct are similar to the ones presented for dry oil in Figure 6.17. The differences between the cases arise in the actual values. They can be inferred from the axial contours located $50 \mathrm{~mm}$ upstream the baseplate, which are presented subsequently together with the contours at the combustion chamber. Regarding the velocity at the axial plane, it is about $5 \mathrm{~m} / \mathrm{s}$ slower than in the Diesel case. 


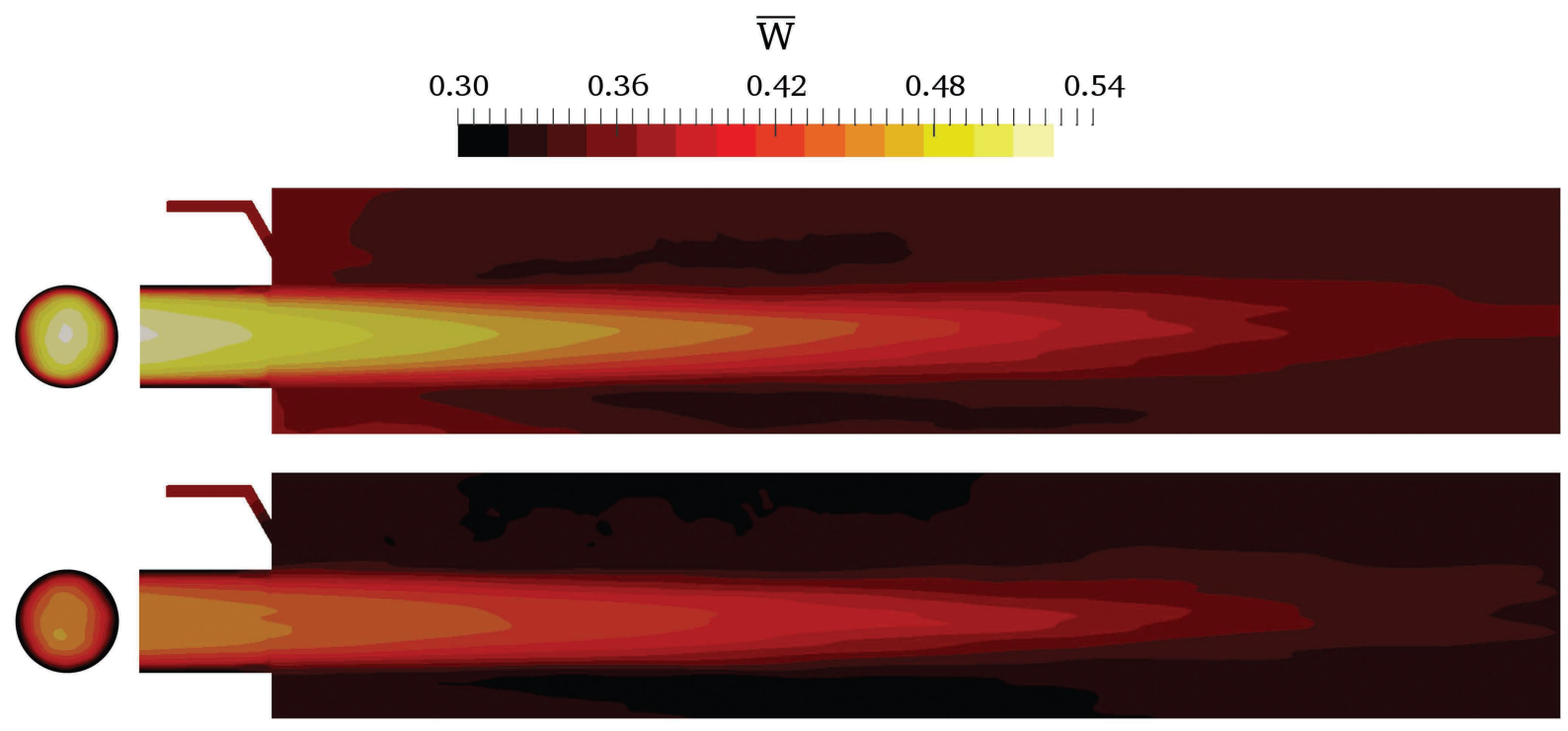

Figure 6.23: Time-averaged gas phase fuel water content at a mid-plane within the combustion chamber for the Emulsion (top) and Preheated (bottom) cases. The axial contours are located $50 \mathrm{~mm}$ upstream of the chamber baseplate.

As displayed in Figure 6.23, the water content is more concentrated at the duct centre than at the wall. Consequently, low water content is found at the jet sides - being smaller for the Preheated case - because the liquid vaporizes earlier and mixes better with the air. If higher preheating temperatures were used, this field would tend to a uniform distribution with the corresponding fully-premixed value.

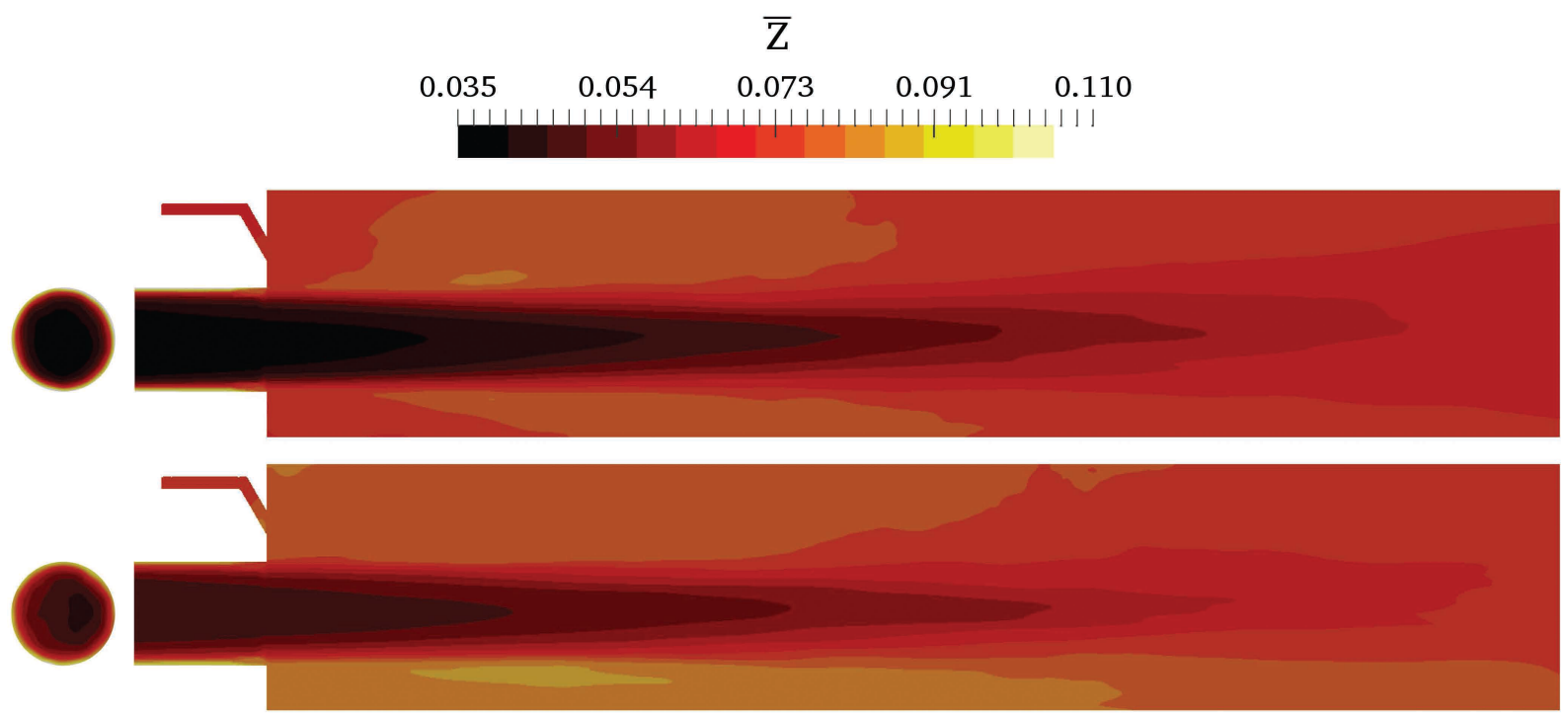

Figure 6.24: Time-averaged mixture fraction at a mid-plane within the combustion chamber for the Emulsion (top) and Preheated (bottom) cases. The axial contours are located $50 \mathrm{~mm}$ upstream of the chamber baseplate.

The mixture fraction is displayed in Figure 6.24. As in the Diesel case, the highest mixture fraction is located at the duct walls. The values of the mixture fraction are significantly higher for emulsions since they include the water mass fraction (see Equation 4.2). 


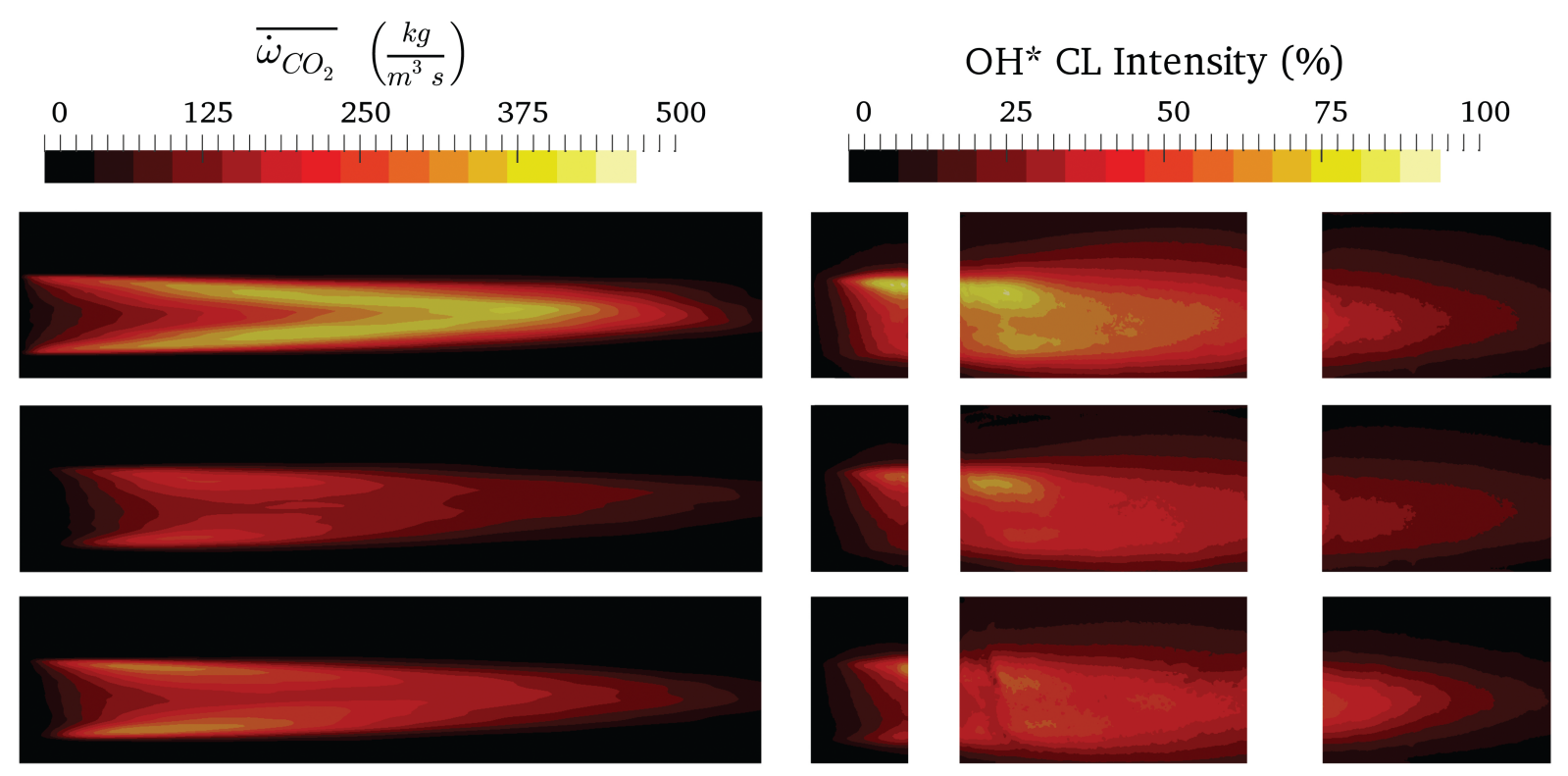

Figure 6.25: Chemiluminescence data (right) and width-integrated progress variable source term (left) for the Diesel (top), Emulsion (middle) and Preheated (bottom) cases.

This increase is compensated by the reduction of the stoichiometric air-to-fuel ratio. As a low water content is present at the wall region, the increased wall impingement results in a more stoichiometric mixture than in the Diesel case. Since the evaporation within the premixing duct is faster for the Preheated case, the mixture fraction within the chamber is higher, especially at the front side.

Figure 6.25 illustrates the flame shape and intensity for the liquid fuel cases. Compared to the gas fuel results in Figure 6.11, excluding the Piloted case, the CFD and OHCL data indicate that the flames are more reactive and stable as the source term is higher and they are more attached to the baseplate. The highest source term is obtained for the dry oil case, but there is more dispersion in the experiments and the maximum source term is located near the baseplate. The latter suggests, as in the Piloted case, that a more adiabatic condition at the baseplate should be used. The source term is reduced when water is added and rises to an intermediate value in the Preheated case. Although the same trends are observed, the change in intensity from the dry oil case is lower in the experiments. This can be attributed to a higher equivalence ratio upstream the flame than in the simulations, caused by the liquid mass flow over-prediction. The lift of the flames in the simulations indicate that the dry oil flame is the most stable, followed by the preheated and thr base wet oil flames. Although the lift is not well captured in the CFD, a similar effect can be observed in the OHCL data on the lower side of the chamber. The kinetic mechanism employed has a strong impact on the results obtained and its further development and validation, especially for the wet cases, is required.

The temperature contours are shown in Figure 6.26. Despite the lower mixture temperature at the axial plane than in the dry oil case, about $50 \mathrm{~K}$ at the centre and $100 \mathrm{~K}$ 


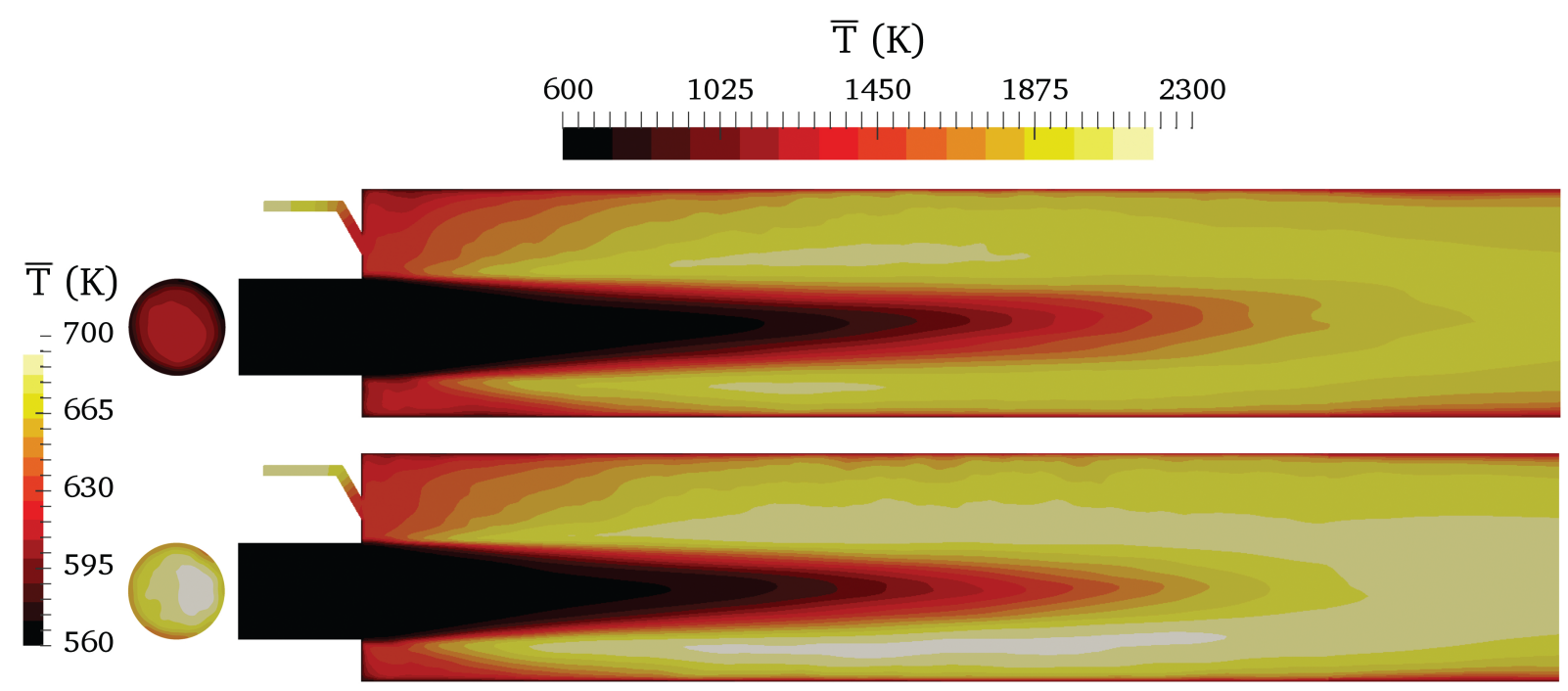

Figure 6.26: Time-averaged gas phase temperature at a mid-plane within the combustion chamber for the Emulsion (top) and Preheated (bottom) cases. The axial contours are located $50 \mathrm{~mm}$ upstream of the chamber baseplate.

at the walls, a higher maximum flame temperature of $100 \mathrm{~K}$ is achieved at the jet sides due to the greater equivalence ratio. This results in a $50 \mathrm{~K}$ higher temperature further downstream. The Preheated case shows an even higher flame temperature at the jet sides. This is mainly attributed to the higher mixture temperature, of about $90 \mathrm{~K}$ larger than in the Emulsion case. The higher mixture fraction and the lower water content - specially at the duct center - also contributes to a larger temperature downstream the flame.

The impact of a lower liquid mass flow coming into the chamber, as observed in the Mie scattering results in Figure 6.21, would result in a higher mixture fraction, lower water content and temperature (and therefore enthalpy) at the exit of the premixing duct. The change in $W$ and $Z$ would tend to increase the local temperature at the sides of the jet, while the lower enthalpy would tend to reduce it. Therefore, despite the over-prediction in the liquid mass flow, it is not expected that the shape of the contours is significantly affected. However, further work on spray and chemistry modelling is required to improve the liquid fuel simulation results, as satisfactory results were obtained for gas combustion with the available data.

\section{References}

[1] W. Meier et al. "Investigation in the TECFLAM swirling diffusion flame: Laser Raman measurements and CFD calculations". In: Applied Physics B: Lasers and Optics 71 (2000), pp. 725-731. 
[2] G. Lartigue, U. Meier and C. Bérat. "Experimental and numerical investigation of self-excited combustion oscillations in a scaled gas turbine combustor". In: Applied Thermal Engineering 24 (2004), pp. 1583-1592.

[3] R. Luckerath et al. "Experimental investigations of flame stabilization of a gas turbine combustor". In: ASME Turbo Expo. 2011.

[4] M. S. Sweeney et al. "The structure of turbulent stratified and premixed methane/air flames I: Non-swirling flows". In: Combustion and Flame 159 (2012), pp. 28962911.

[5] M. S. Sweeney et al. "The structure of turbulent stratified and premixed methane/air flames II: Swirling flows". In: Combustion and Flame 159 (2012), pp. 29122929 .

[6] O. Lammel et al. "Experimental Analysis of Confined Jet Flames by Laser Measurement Techniques". In: Journal of Engineering for Gas Turbines and Power 134 (2012), p. 041506.

[7] W. Meier et al. "Raman/Rayleigh/LIF measurements in a turbulent CH4/H2/N2 jet diffusion flame: experimental techniques and turbulence-chemistry interaction". In: Combustion and Flame 123 (2000), pp. 326-343.

[8] A. R. Masri, R. W. Bilger and R. W. Dibble. "The local structure of turbulent nonpremixed flames near extinction". In: Combustion and Flame 81 (1990), pp. 260276.

[9] P. Griebel, P. Siewert and P. Jansohn. "Flame Characteristics of Turbulent Lean Premixed Methane/Air Flames at High Pressure: Turbulent flame speed and flame brush thickeness". In: Proceedings of the Combustion Institute. Vol. 31. 2. 2007, pp. 3083-3090.

[10] R. Lückerath, W. Meier and M. Aigner. "FLOX®Combustion at High Pressure With Different Fuel Compositions". In: Journal of Engineering for Gas Turbines and Power 130.1 (2007), p. 011505.

[11] S. Dederichs, N. Zarzalis and C. Beck. "Validation of a Novel LES Aproach Using Tabulated Chemistry for Thermoacoustic Instability Prediction in Gas Turbines". In: ASME Turbo Expo. 2015.

[12] A. R. Masri and J. D. Gouder. "Turbulent Spray Flames of Acetone and Ethanol Approaching Extinction”. In: Combustion Science and Technology 182 (2010), pp. $702-715$.

[13] F. L. Dryer. "Water addition to practical combustion systems - Concepts and applications". In: Symposium (International) on Combustion 16 (1977), pp. 279-295. 
[14] S. R. Gollahalli, M. K. Nasrullah and J. H. Bhashi. "Combustion and emission characteristics of burning sprays of a residual oil and its emulsions with water". In: Combustion and Flame 55.1 (1984), pp. 93-103.

[15] J. M. Ballester, N. Fueyo and C. Dopazo. "Combustion characteristics of heavy oil-water emulsions". In: Fuel 75.6 (1996), pp. 695-705.

[16] D. G. Goodwin, H. K. Moffatt and R. L. Speth. Cantera: An Object-oriented Software Toolkit for Chemical Kinetics, Thermodynamics, and Transport Processes. Version 2.1.2. 2014.

[17] G. P. Smith et al. GRI-Mech 3.0. The Gas Research Institute. URL: http://www . me.berkeley.edu/gri_mech/.

[18] J. Biet et al. "Experimental and modeling study of the low-temperature oxidation of large alkanes". In: Energy \& Fuels 22 (2008), pp. 2258-2269. 


\section{Chapter 7}

\section{Numerical investigation of the models' acoustic response}

\subsection{Introduction and literature review}

The interaction between pressure waves and unsteady heat release can drive the oscillations, resulting in noise and vibration that can potentially damage the engine. The analysis and understanding of flame dynamics (i.e. thermo-acoustic interaction) is essential for the development of advanced gas turbine engines.

A common method to analyse the thermo-acoustic stability of a system is the forced response approach, where the flame is excited artificially and the response obtained determines the Flame Transfer Function [1,2], which helps to describe the system's dynamics and to control the instabilities. The FTF provides a relationship between the heat release and the velocity or pressure fluctuations. They can be obtained by detailed, unsteady Computational Fluid Dynamics that must resolve the thermo-acoustic coupling mechanism. The best suited method for this is the Large-Eddy Simulation.

Thermo-acoustics with gaseous fuels have been extensively studied. Lieuwen et al. [3] reviewed the mechanisms for combustion instabilities in lean premixed burners, where equivalence ratio fluctuations due to acoustic perturbations are found as the main driving force. The interaction and characterization of pressure oscillations that results in a change of the fuel flow rate, in terms of time delays, are investigated by Lieuwen et al. [4]. The thermo-acoustic stability analysis of a laminar premixed burner is examined by Kaess et al. [5], where the validation of the CFD-models against experimental data is also performed. Additionally, investigations on turbulent combustion in a Siemens hybrid burner were carried out by Giauque et al. [6].

Few acoustic studies for spray flames can be found in the literature. The impact of forced excitation on the primary breakup and secondary atomization has been experimentally examined by Anderson et al. [8], who provided information on the acoustic 
response for the investigated fuel injector. The addition of acoustic perturbations to a liquid fuel jet in cross flow was investigated by Anderson et al. [9]. Song et al. [10] stated that the liquid jet atomization is enhanced by an oscillating cross flow, resulting in a greater number of smaller droplets (i.e. lower SMD). For the droplet breakup and evaporation, investigated by Cossali et al. [11] and Moon et. al [12], the droplet surface shape is strongly influenced by acoustic waves and consequently the evaporation is enhanced. Ha [7] investigated the evaporation and combustion of a single droplet under oscillating flow. The magnitude of the relative velocity between air and droplet, due to the acoustic excitations, was found to increase the heat and mass transfer in the evaporation process. Furthermore, droplet combustion times were smaller for increasing amplitudes. The impact of acoustic oscillations on spray combustion has been numerically analysed by Zhu et al. [13]. He found out that smaller droplets are formed in the process of atomization. Therefore, the evaporation time is reduced and the amount of vaporized fuel supplied to the flame increases, which has a strong influence on the combustion stability.

This chapter focuses on the acoustic response of the atomization, evaporation and combustion models to forced excitations. Firstly, simple test cases are simulated to analyse the response of the models individually and coupled to each other. Acoustic simulations of the jet flame are subsequently performed with both gas and liquid fuels. Since there is no experimental data available, this work is a feasibility study on the solver capabilities to simulate acoustic effects.

\subsection{Approach}

To analyse numerically the acoustic response of a model or a configuration, the selection of the inlet and outlet boundaries that limit the domain is important. A forced excitation, with a certain frequency and amplitude, is applied to the inlet flow field using an oscillating velocity boundary condition. This fluctuation changes the inlet mass flow, but also affects the pressure field so that sinusoidal waves travel through the domain and reach the outlet. These waves could be partially reflected, if there are geometrical area changes, and would eventually arrive at the inlet. At the location of the numerical boundaries, since they do not exist physically, a wave should leave the domain without any reflection. Thus, following Poinsot and Veynante [14], non-reflecting boundary conditions are used for the inlet and the outlet boundaries to reduce the amplitude of the waves that are reflected. They are based on the LODI approach [15], which consists in imposing the far-field pressure or velocity conditions at a certain characteristic distance from the actual patches. The latter implies that a larger distance results in a lower wave reflection, but a drift from the mean value imposed arises. The characteristic distance is selected based on the excitation frequency, so that proportionally higher values are used for larger wave lengths. 
Table 7.1: Relevant conditions imposed on the inlet and outlet boundaries as well as on the injected parcels in each simulated case.

\begin{tabular}{c||ccc|ccc} 
& Atomization & $\begin{array}{r}\text { 1-D droplet } \\
\text { evaporation }\end{array}$ & $\begin{array}{c}\text { 1-D } \\
\text { combustion }\end{array}$ & $\begin{array}{c}\text { Evaporating } \\
\text { spray }\end{array}$ & $\begin{array}{c}\text { 1-D spray } \\
\text { flame }\end{array}$ & $\begin{array}{c}\text { Jet } \\
\text { flame }\end{array}$ \\
\hline \hline$T_{\text {in }}(\mathrm{K})$ & 293 & 700 & 600 & 700 & 700 & 700 \\
$\bar{U}_{\text {in }}(\mathrm{m} / \mathrm{s})$ & 100 & 10 & 0.71 & 100 & 0.964 & 5.8 \\
$U_{\text {in }}^{\prime}(\mathrm{m} / \mathrm{s})$ & 20 & 2 & 0.142 & 20 & 0.193 & 1.45 \\
\hline$P_{\text {out }}(\mathrm{bar})$ & 8 & 8 & 8 & 8 & 8 & 8 \\
\hline$d_{l, \text { inj }}(\mu \mathrm{m})$ & 450 & 100 & - & 450 & 30 & 500 \\
$U_{l, \text { inj }}(\mathrm{m} / \mathrm{s})$ & 45 & 10 & - & 25 & 0.71 & 20 \\
$T_{l, \text { inj }}(\mathrm{K})$ & 293 & 293 & - & 293 & 293 & 293
\end{tabular}

Six configurations are considered: one for each individual model (atomization, evaporation and combustion) that has been presented in Chapters 2-4, and three for the coupled configurations that have been simulated in Chapters 5 and 6. Hexadecane (without any water addition) is used as the liquid fuel, as in Chapter 5; except for the jet flame where both methane and DF2 are employed, as in Chapter 6. The boundary as well as the injection conditions used in each test case are exposed in Table 7.1, being the pressure level imposed in all the simulations around 8 bar at the outlet boundary, as in Chapter 6 . The inlet velocity fluctuations are set about 20-25\% the mean velocity and the investigated forcing frequencies range from $100 \mathrm{~Hz}$ to $1000 \mathrm{~Hz}$ in $100 \mathrm{~Hz}$ steps, except for the Jet Flame case where simulations are performed for four frequencies: 100, 200, 300 and $800 \mathrm{~Hz}$.

The effect of these acoustic perturbations on a model is described in terms of transfer functions. The latter are determined by monitoring the relevant model characteristics and, after applying the Hanning window to the signals, calculating their Fourier Transform. The amplitude at the forcing frequency is extracted and normalized by the pressure amplitude at a reference position. That location is the inlet boundary for all the simulations except for the Jet flame case, which is placed upstream of the gas injection so that the acoustic path through the S-turn is skipped in the analysis. Regarding the phase difference between each variable and the reference pressure signal, which is also extracted from the Fourier Transform, a phase multiple of $360^{\circ}$ is subtracted (wrap-around) so that it decreases with the forcing frequency. In this way, the time lag is defined in Equation (7.1) as the local slope of the phase curve. A higher time lag can be interpreted as a slower response to the inlet excitation.

$$
\text { Phase } \propto-\tau F
$$


In the present work, the wrap-around is not applied if the phase is at least $20^{\circ}$ lower than the phase of the previous frequency. The same approach is to the Jet flame case, although there are potentially several wrap-arounds between 300 and $800 \mathrm{~Hz}$ that are missed because the intermediate frequencies are not simulated.

For the simulations of a jet in cross flow atomization, the rectangular channel is the same as the described in Section 2.3. In this case, the mesh with only one refinement region around the injection point has been used. The jet is injected perpendicular to the cross flow and the mass flow is calculated so that the initial momentum ratio is approximately 12 . The parameters used to determine the model response are the spray penetration, the liquid flux and the SMD at a plane located $80 \mathrm{~mm}$ downstream of the injection point.

For the droplet evaporation case, the relative velocity has a big impact on the evaporation rate. Therefore, unlike in Section 3.3 where the droplet was fixed at the centre of a box, in this study the droplet is able to move. It is injected into a 1-D domain, which has a cell size of $1 \mathrm{~mm}$, with the same velocity as the mean gas phase velocity. The surface regression rate, defined in Equation (7.2), is tracked during the entire droplet lifetime.

$$
S R R=\frac{d\left(d^{2}\right)}{d t}
$$

For the combustion model, 1-D premixed flames with an equivalence ratio of 0.8 are simulated. The inlet mean velocity is set to the laminar flame speed, while a $100 \mathrm{~K}$ lower inlet temperature than in the 1-D droplet evaporation case is used as a rough estimation of the temperature drop due to evaporation and mixing upstream of the flame. The kinetic mechanism from Biet et al. [16] is used to generate the chemistry tables. A longer domain than in Section 4.3 is used (about $0.2 \mathrm{~m}$ ), since the flame position oscillates more than for steady state, but cell size is maintained. The volume-integrated heat release, flame thickness and flame speed are monitored for the acoustic analysis. The flame thickness is calculated by sampling two iso-surfaces at $c=0.01$ and $c=0.99$ through field interpolation. In this case, the flame speed cannot be computed with Equation (4.18) since the velocity and density change spatially due to the acoustic waves. Thus, the velocity is sampled at the $c=0.01$ plane and the flame speed is calculated from the change in the sampling position:

$$
S u^{\prime}=U_{c=0.01}-\frac{\Delta x}{t_{\text {sampl }}}
$$

Acoustic simulations of evaporating sprays, where the atomization and evaporation models are coupled, are also performed. The same momentum ratio as in the Atomization cases is targeted with the reduction in the injection velocity. Apart from the spray characteristics, the area-averaged mixture fraction at the outlet boundary (located at $150 \mathrm{~mm}$ downstream of the injection point) is also post-processed. 
For the 1-D spray flame case, in which the evaporation and combustion models are coupled, the same mesh and mean equivalence ratio as in the 1-D combustion case is used. Small droplets are injected near the inlet with a velocity equal to the mean air inlet velocity. The flames are initialized downstream of the evaporation distance (anchored flames) and they are affected by the mixture fraction fluctuations, from the spray, as well as by the acoustics. As in Section 5.2, chemistry tables are created for several equivalence ratios and enthalpies based on an initial simulation. Apart from the variables used in the 1-D combustion case, the fuel mass fraction and the mixture enthalpy upstream of the flame $(c=0.01)$ are also sampled to the determine their transfer function. Acoustic simulations of 1-D saturated flames, where the droplets interact directly with the flame, are out of the scope of this work since they might be too complex or unstable.
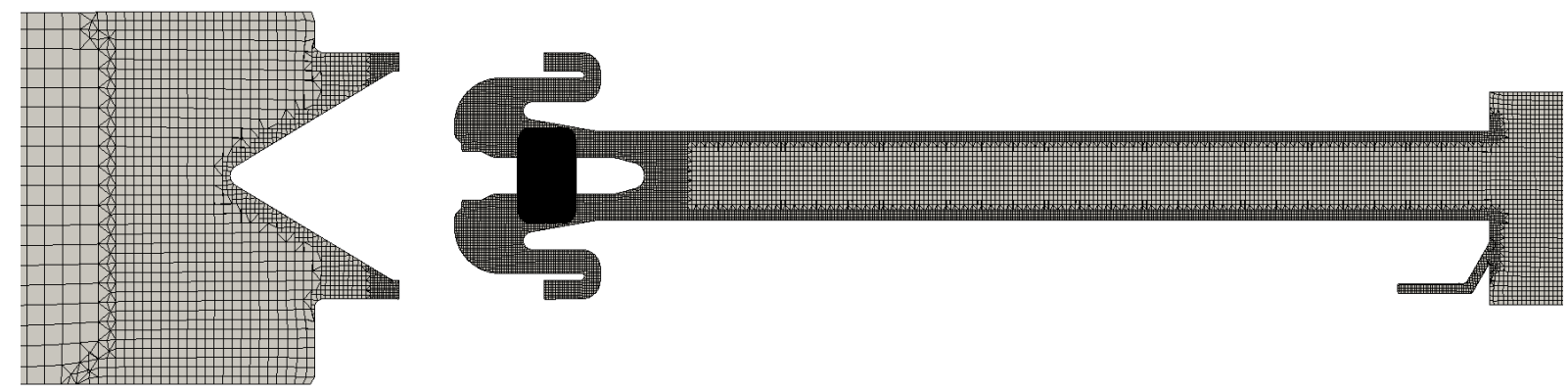

Figure 7.1: Computational mesh used for the simulation of the Jet Flame case.

Regarding the jet flame acoustic simulations, the inlet plenum has been extended by $1 \mathrm{~m}$ and the outlet has been set $5 \mathrm{~mm}$ upstream of the combustion chamber nozzle (i.e. the outlet plenum have been removed). In this way, there is a significant distance between the inlet/outlet boundaries and the geometrical area changes. The mean air inlet velocity is obtained from the results obtained in Section 6.4.1 and the fuel mass flows are the same as for the Baseline and Diesel cases from Chapter 6, which are included in Table 6.1, for the liquid and gas simulations respectively. The wall temperature distribution defined in Equation (6.1) is also imposed at both the baseplate and the chamber walls. In the present simulations, if the local enthalpy falls below the lowest enthalpy in the tables, the source term is not set to zero (as in the previous chapter) but the closest enthalpy value is used. This implies that the flames for both the liquid and gas fuels are attached to the baseplate (i.e. they are not lifted) so that the acoustic effects on the flames can be compared more clearly. The mesh used is illustrated in Figure 7.1 and it has about 1.8 million cells. In comparison with the mesh presented in Figure 6.4, the cell size at the Sturn, within the premixing duct and the front side of the combustion chamber is double. A comparison between the results obtained with both meshes for the non-excited gas and oil cases is included in Appendix F. Despite the significant discrepancies, especially between the oil simulations, this coarsening is kept to reduce the computational cost since the simulation time is much larger than for a steady state case. During run time, the $c=0.5$ 
flame iso-surface is used to calculate an area-averaged enthalpy and mixture fraction. The area of this iso-surface and the volume-integrated heat release are also obtained. Additionally, the spray SMD and mass flow at three axial planes located 120, 250 and $380 \mathrm{~mm}$ downstream of the injection point, being the last one at the combustion chamber entry, are also recorded. The Fourier transform is applied to all these variables. Since the flame thickness and flame speed vary along the flame surface and are more difficult to calculate in a 3-D configuration, they are not considered in the acoustic analysis of this case.

The spatial and temporal schemes used are the same as for the steady simulations carried out in previous chapters. Furthermore, a lower time step is used to decrease numerical acoustic damping. The acoustic CFL number of 0.7 , recommended by Krediet [1], is however exceeded in order to limit the computational effort.

\subsection{Results}

\subsubsection{Atomization}

The primary atomization of a liquid jet in cross flow depends greatly on the relative velocity between the liquid and the gas phases. Particularly, for a constant liquid mass flow, the momentum ratio is defined based on the square of this velocity (see Equation (2.5)). It influences the amount of mass stripped from the jet and its associated diameter, as well as the column break-up point. Additionally, the secondary break-up process is governed by the Weber number, which determines the growth of the oscillations on the droplet surface $[17,18]$ :

$$
W e=\frac{\rho_{g} U_{r e l}^{2} d}{\sigma_{l}}
$$

For the forcing velocity amplitude specified in Table 7.1, the pressure amplitude at the inlet boundary is around 600 mbar. Figure 7.2 shows the impact of the oscillations with several frequencies on the depth-integrated liquid flux and SMD distributions, which are time-averaged during $50 \mathrm{~ms}$, at the post-processing plane. The non-excited solution is also plotted as reference. For $100 \mathrm{~Hz}$ the spray penetration, in terms of the maximum height with significant flux, and dispersion are greatly improved while the maximum volume flux value is located at the same height. The size of droplets stripped from the jet and from the primary droplets are larger, but the SMD is lower at the high volume flux region. As the excitation frequency increases, the spray dispersion and penetration are reduced, being the latter slightly lower than in the reference solution. The SMD decreases too, especially at the zone closer to the bottom wall and at the maximum volume flux position. The latter indicates that the secondary atomization is enhanced when forced excitation is introduced, which is in agreement with the findings from the work of Song 

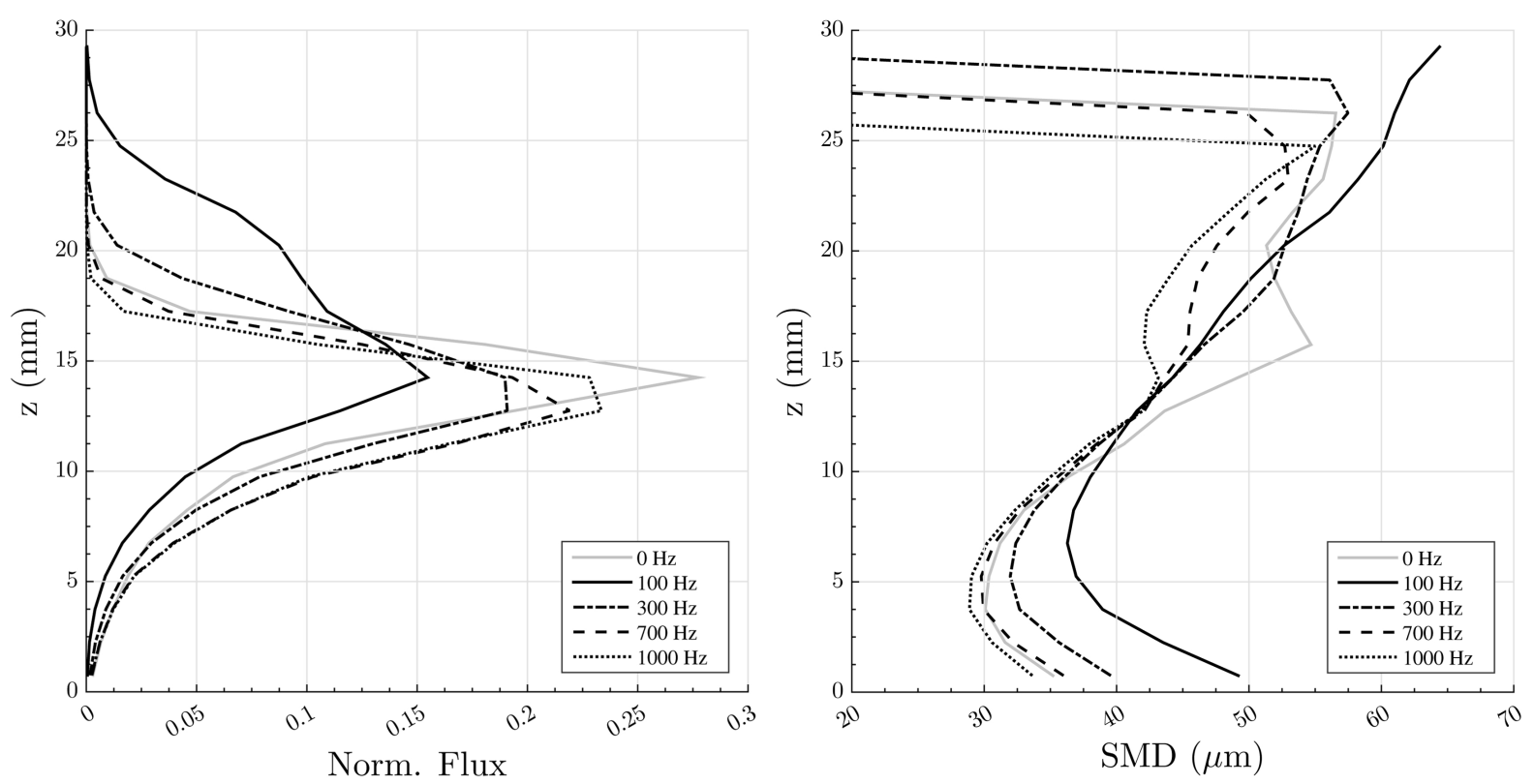

Figure 7.2: Effect of the forcing frequency on the depth-integrated flux and SMD profiles at a plane $80 \mathrm{~mm}$ downstream the injection point.

and Ramasubramanian [10].

To determine the spray response, the volume flux and SMD distributions are also integrated over the height (to obtain a global value) while the location of the maximum flux is taken as the penetration. The Bode diagram is displayed in Figure 7.3, where the amplitude and the phase of each variable's oscillations are plotted over the excitation frequency. It shows that the SMD is less sensitive to acoustic oscillations and its amplitude decreases with the frequency. The same trend is obtained for the penetration, but with a higher amplitude. The highest response is observed for the liquid flux, which implies that zones with high and low amount of liquid are created by the acoustic waves. Unlike the other variables, the amplitude increases from 100 to $800 \mathrm{~Hz}$ and it drops slightly afterwards. The phase plot shows a similar time lag between both the SMD and the flux, but higher than the one for the spray penetration.

\subsubsection{1-D droplet evaporation}

The droplet evaporation process is governed by the heat transferred from the gas to the droplet and the amount of mass evaporated. The former depends on the gas phase properties, the intensity of the convective field and the gas-liquid temperature difference. The latter depends on the droplet temperature (which is determined from the time-evolution of the heat balance), pressure level and the gaseous fuel concentration at the droplet surface. In general, the addition of a forced excitation to the mean air flow velocity results in a faster heating period and therefore in a shorter evaporation time [7]. Figure 7.4 proves that this effect is also obtained in the simulations. The droplet lifetime, 

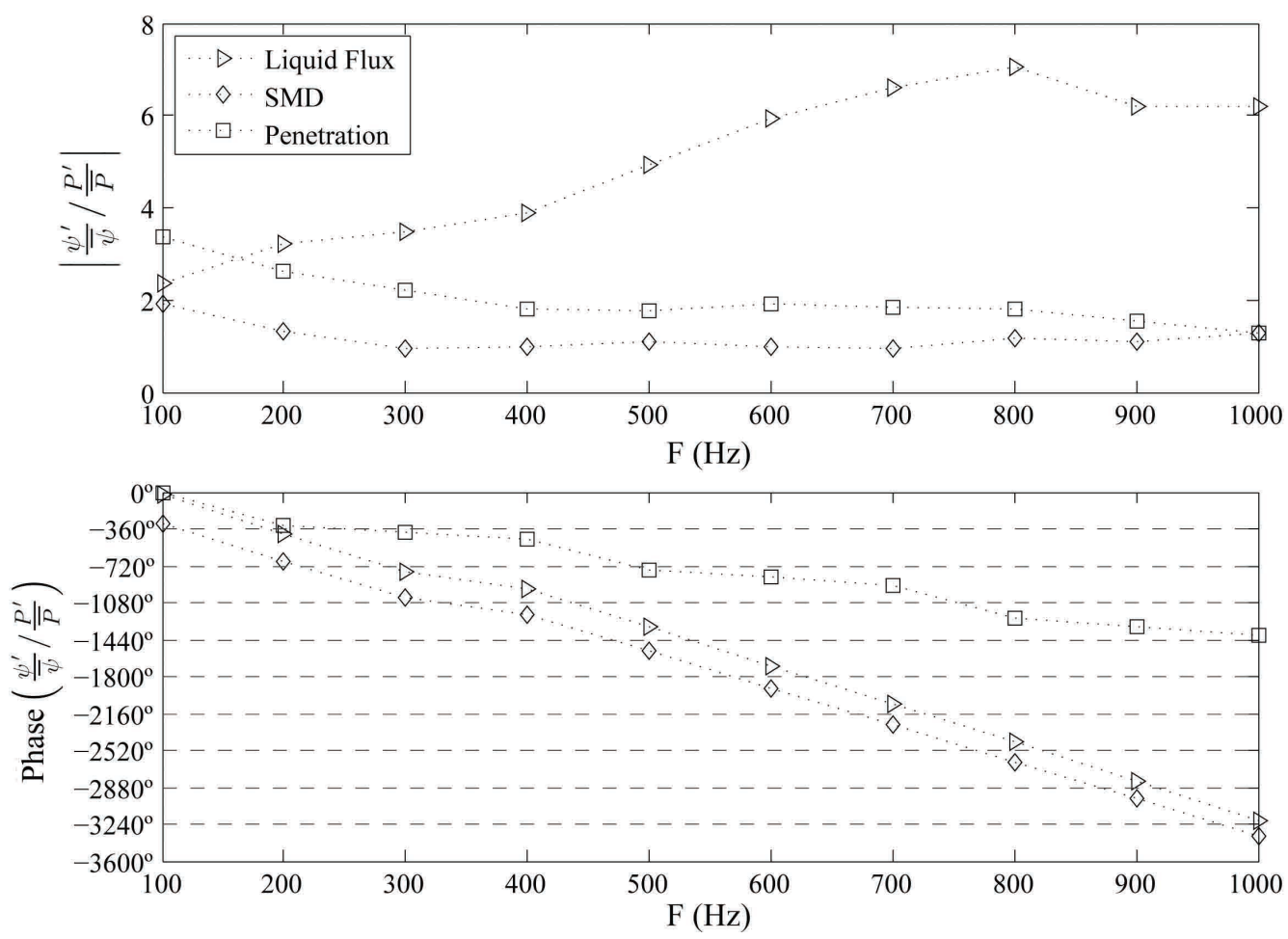

Figure 7.3: Bode diagram of the characteristic droplet size and spray penetration transfer functions.

non-dimensionalized with the non-excited value, is plotted versus the forcing frequency. The lowest value is obtained for $100 \mathrm{~Hz}$ and it increases linearly until $400 \mathrm{~Hz}$. The latter can be considered as the cut-off frequency, since the evaporation time does not change significantly for higher frequencies.

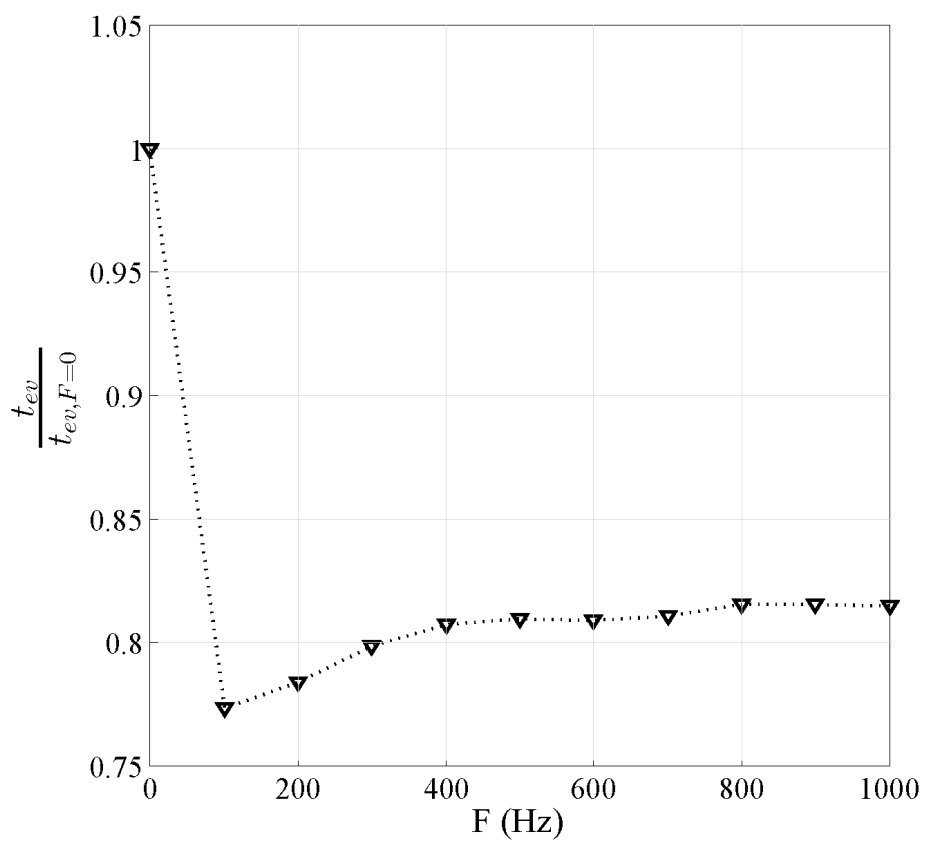

Figure 7.4: Evolution of the droplet evaporation time with the forcing frequency. 

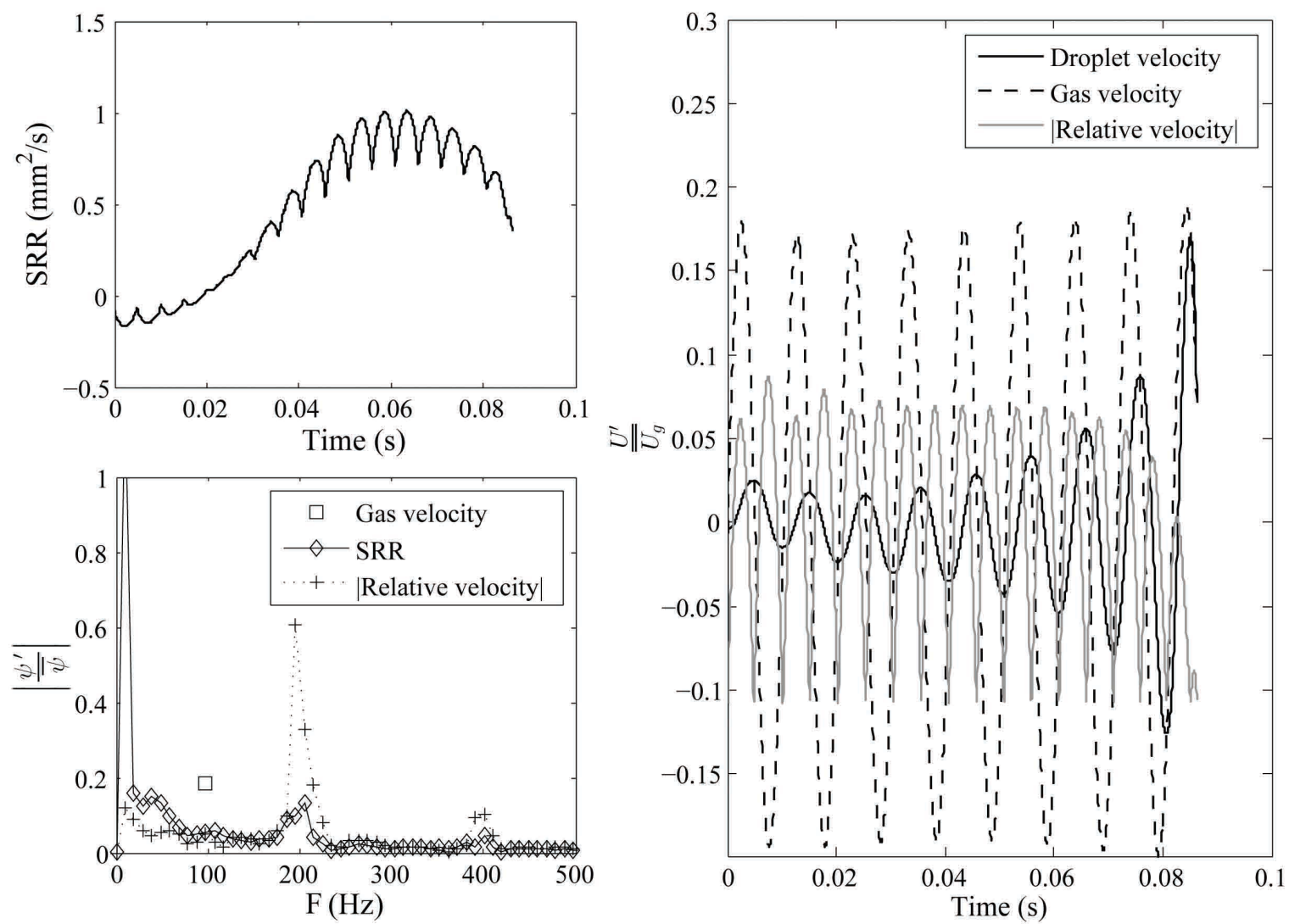

Figure 7.5: $100 \mathrm{~Hz}$ forcing of a single droplet: SRR time series (top left); Fourier transform of the SRR, gas velocity and magnitude of the relative velocity (bottom left); fluctuations time series for the droplet velocity, gas phase velocity and magnitude of the droplet relative velocity non-dimensionalized with the mean gas velocity (right).

Results for $100 \mathrm{~Hz}$ have been chosen to exemplify the effect of forced excitations on the evaporation model. The temporal evolution of the droplet surface regression rate is shown on the upper left plot of Figure 7.5. At the initial heating stage, the SRR is negative (i.e. the droplet expands) because the liquid density decreases. As the droplet temperature increases, the SRR raises accordingly. A maximum value is reached for both quantities, after which they decrease until the droplet is completely vaporized. This droplet temperature and SRR decrements are caused by the reduction of the relative velocity, since the droplet has less inertia and it is able to respond faster to the gas phase oscillations. The latter can be observed in the time series of the droplet velocity, air velocity and the absolute value of the relative velocity (non-dimensionalized with the mean air velocity) depicted on the right graph.

At the beginning the phase between the droplet and the air velocity fluctuations is $90^{\circ}$. As the droplet gets vaporized, the frequency and amplitude of the droplet velocity increase so that it gets in phase with gas phase velocity fluctuations. The relative velocity fluctuations have about double the frequency of the forced excitation, since they are calculated from the absolute value of the difference between the air and droplet ve- 
locities. Correlations for the convective mass and heat transfer, in terms of Sherwood and Nusselt numbers respectively, are strongly influenced by the Reynolds number (see Equation (3.5)), which is calculated from the relative velocity. Thus, the heat transferred to the droplet oscillates with double the frequency of the inlet excitation and therefore the SRR as well. This is shown in the lower left graph, where the Fourier Transform of previous quantities are plotted. Particularly, the SRR shows a low frequency peak, which corresponds to the frequency of the droplet lifetime, and a second peak at the frequency of the relative velocity. The same behaviour is obtained for higher forcing frequencies, and since it is non-linear the Bode diagram is not plotted for this process.

\subsubsection{1-D combustion}

For this case the velocity oscillations are so small that the amplitude of the pressure waves are about 5 mbar and the inlet temperature oscillations can be neglected. Thus, only velocity oscillations have an effect on a laminar premixed flame.

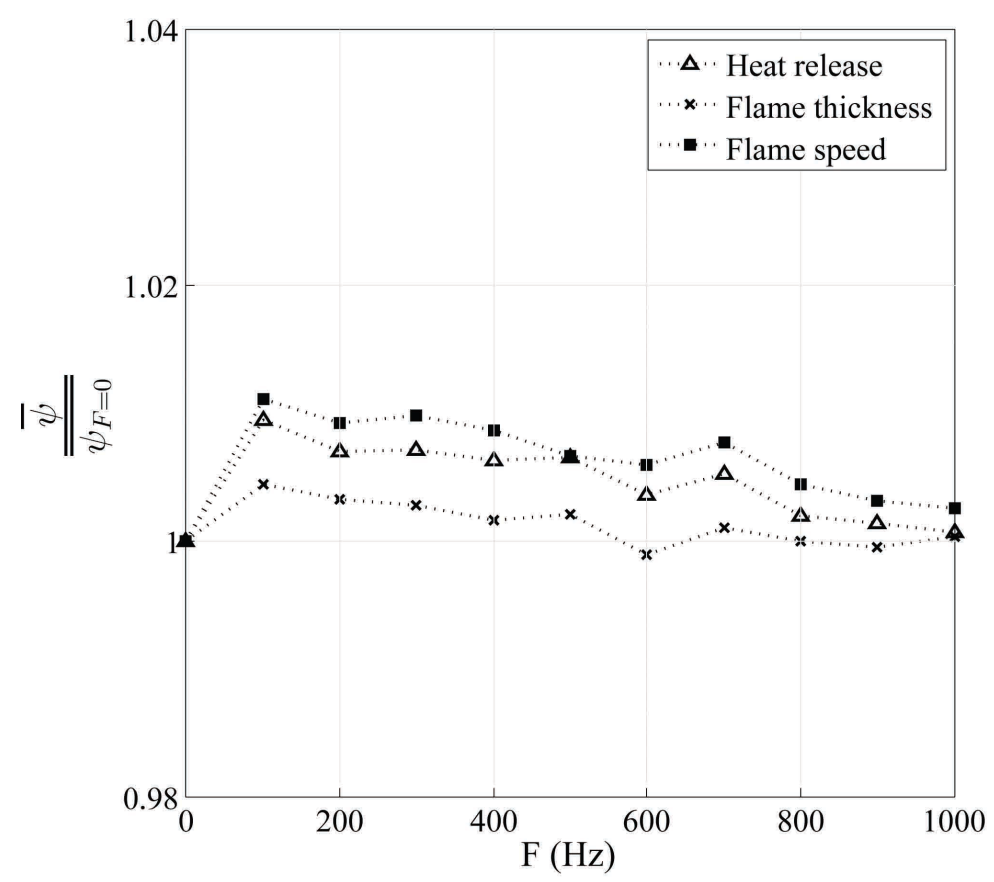

Figure 7.6: Evolution of the time-averaged flame speed, flame thickness and volumeintegrated heat release with the forcing frequency.

When the local velocity upstream of the flame front is higher than the flame speed, the flame moves backwards and the flame gets thicker due to the higher convective transport of the governing species. The opposite is true for local velocities lower than the flame speed. The heat release depends on the amount of fuel mass flow being consumed, which oscillates with the velocity fluctuations since the unburned density and the fuel mass fraction are constant. In turn, heat release fluctuations induce oscillations in the laminar flame speed. The mean values of these variables, non-dimensionalized with the results 

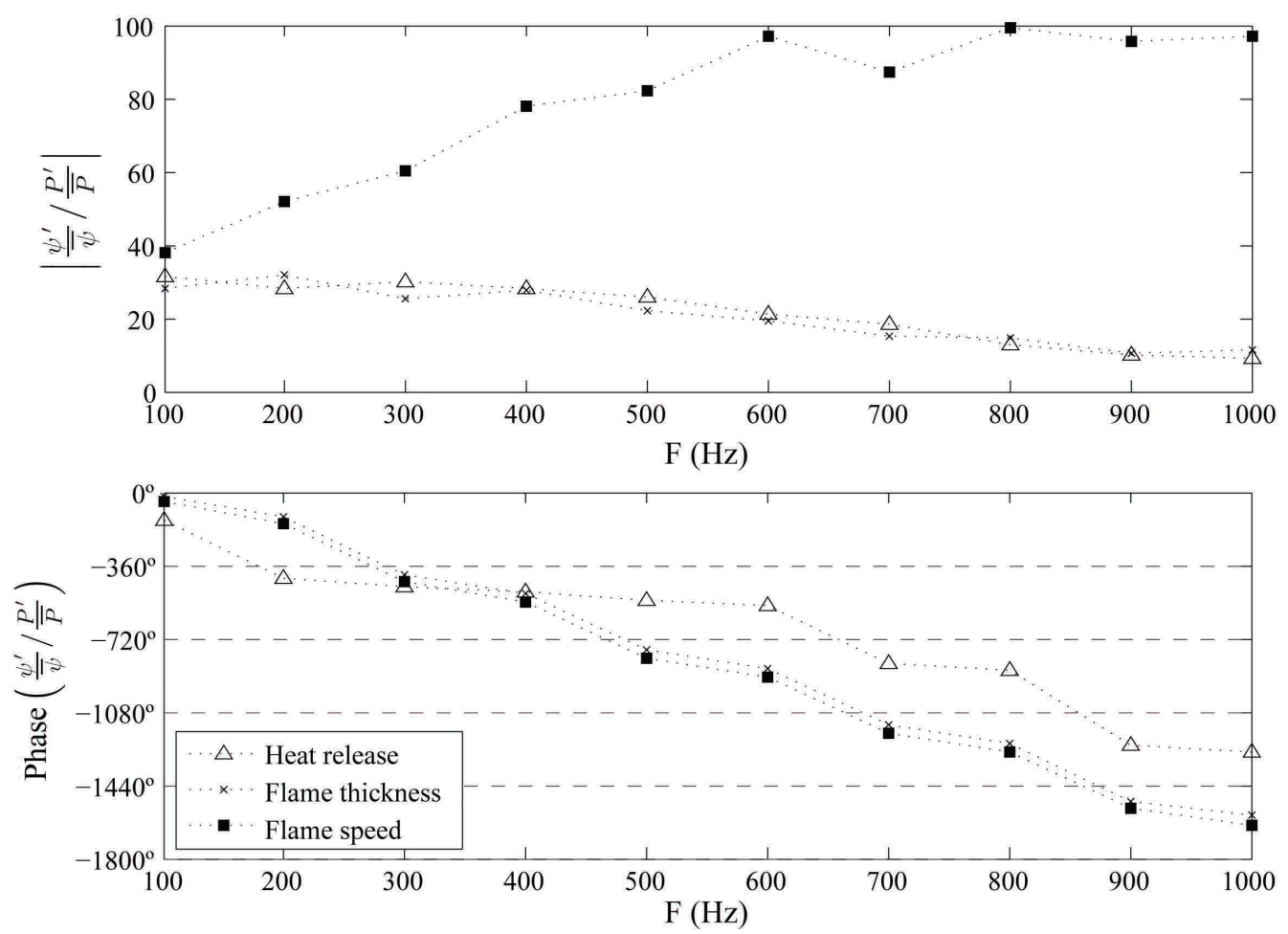

Figure 7.7: Bode diagram of the flame speed, the flame thickness and the volumeintegrated heat release transfer functions.

from a non-excited simulation, are shown in Figure 7.6 as a function of the frequency. The largest increase is found at $100 \mathrm{~Hz}$, from which they decrease. Since the variations are about $1 \%$ or less, it can be assumed that acoustics only cause fluctuations in the monitored variables but not in their time-averaged values.

The Bode diagram in Figure 7.7 indicates that the flame speed is more sensitive to the flow oscillations. Its amplitude increases with the forcing frequency, being the slope smaller for the higher frequencies. Conversely, a similar amplitude level is obtained for the flame thickness and the volume-integrated heat release with the same decay evolution with the frequency. The lower plot shows that, despite the $360^{\circ}$ wrap-arounds, the phase difference between the three variables is small. Thus, the time lag is the same for the flame speed and the flame thickness while it is slightly lower for the heat release.

\subsubsection{Evaporating spray}

The evaporation and combustion models are combined to simulate an evaporating spray. The response of this configuration is likely have a linear component, i.e. an amplitude peak at the same frequency as the excitation signal, with super-imposed non-linear components at the harmonics of the forcing frequency. For the spray variables, a similar response as in Section 7.3.1 is expected. For the area-averaged fuel mass fraction, the amplitude of the first mode is mainly caused by the liquid flux and SMD oscillations at the same frequency. The non-linear response of the evaporation process contributes to the amplitude of the 


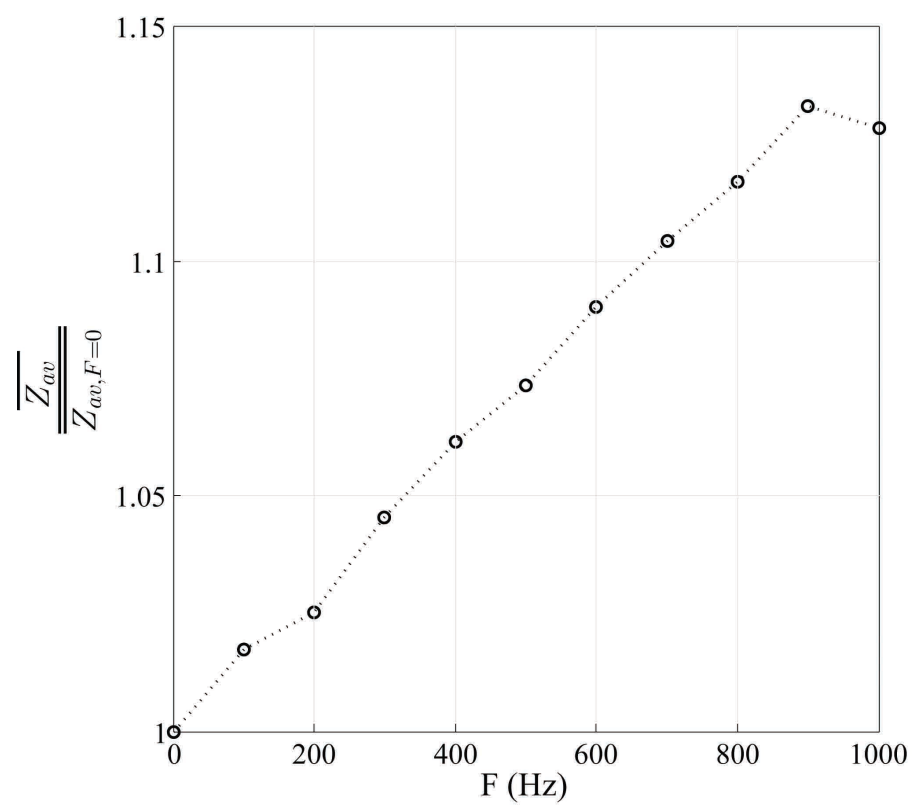

Figure 7.8: Time-averaged fuel mass fraction related to time average fuel mass fraction for $\mathrm{F}=0 \mathrm{~Hz}$ over forcing frequencies.

second mode. As aforementioned, only the first mode is analysed.
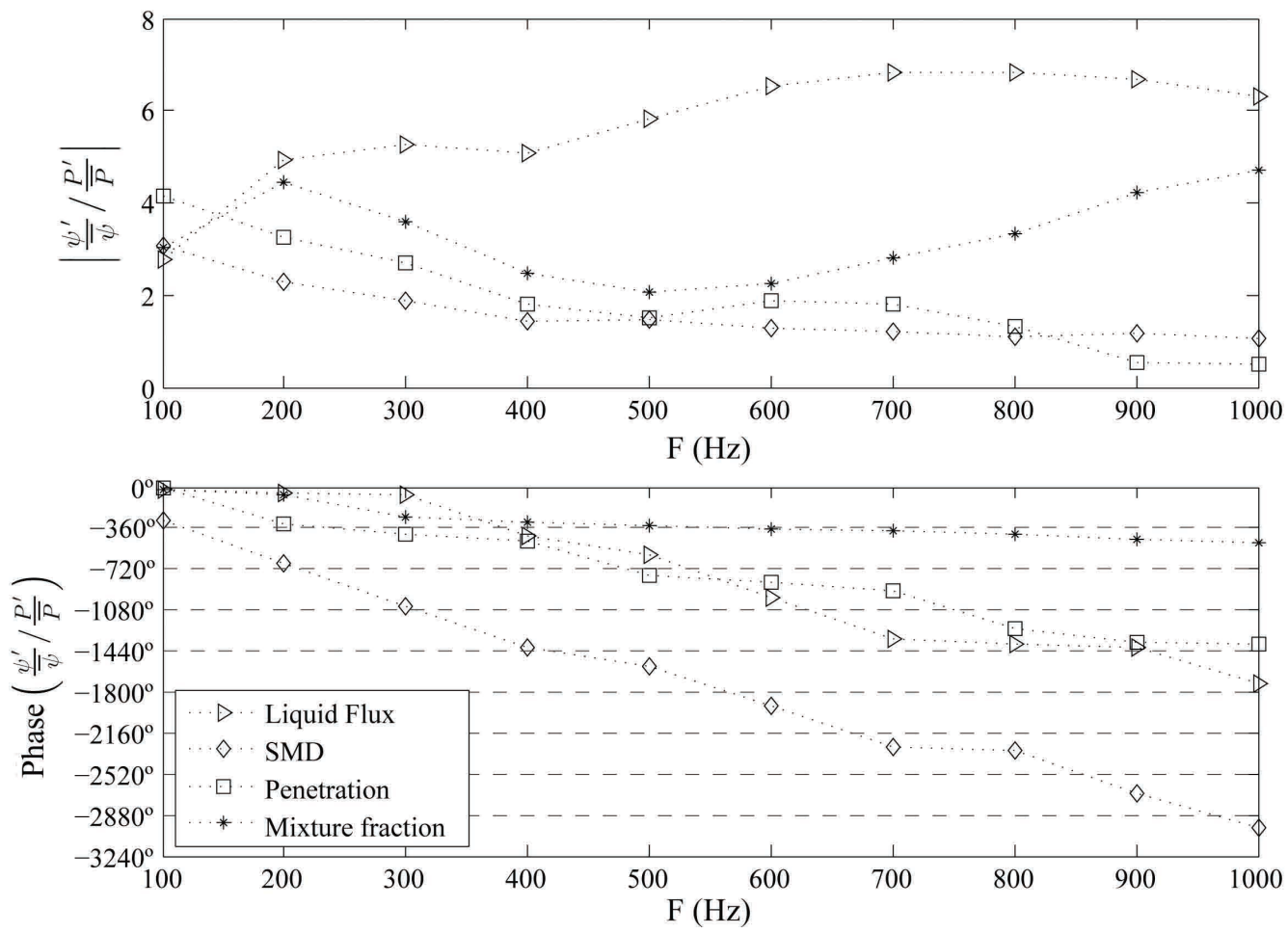

Figure 7.9: Bode diagram of the characteristic droplet size and penetration transfer functions of an evaporating spray in cross flow.

The effect of acoustic waves on the mean spray statistics has been shown in Section 7.3.1. The change in the area-averaged fuel mass fraction at the outlet boundary with the forcing frequency is plotted in Figure 7.8. The values are non-dimensionalized 
with the time-averaged value of the non-excited solution, which can be obtained from Figure 5.1. The reduced flux-averaged SMD and the enhanced evaporation rate with the frequency leads to a linear rise of the averaged mixture fraction. The small variation from 900 to $1000 \mathrm{~Hz}$ suggests that there is a cut-off effect around this point, so that the amount of evaporated mass may reduce significantly at higher frequencies.

Figure 7.9 presents the response of the spray statistics and the fuel mass fraction in a Bode diagram. The inlet pressure amplitude is around $430 \mathrm{mbar}$, which is lower than in the Atomization case with the same velocity fluctuations due to the smaller air density. As in Section 7.3.1, the amplitude is larger for the liquid flux than for the penetration, which is in turn greater than for the SMD. The mixture fraction oscillations have a larger amplitude than the penetration, but lower than the flux. After an initial increase from 100 to $200 \mathrm{~Hz}$, it decreases for intermediate frequencies and rises at larger frequencies. The phase plot also shows the same trends as in Section 7.3.1, having the spray penetration a lower time lag than the SMD and the liquid flux. The mixture fraction displays a smaller time lag than the spray penetration.

\subsubsection{1-D spray flame}

The influence of acoustic excitations on the coupling between the evaporation and the combustion models is analysed in this section. As aforementioned in Section 7.3.2, the inflow oscillations influence the droplet evaporation by means of an increased heat transfer due to the relative velocity. The interaction between the droplet acceleration, because of the drag force, and the droplet inertia leads to a non-uniform droplet distribution. Hence, similarly to the liquid flux in the acoustic spray simulations, spatially lean and rich zones arise within the domain due to agglomerations of droplets. The irregular droplet distribution results induces oscillations in the vaporized fuel mass fraction and in the mixture enthalpy. Since these variables are used by the combustion model to read the progress variable source terms, the heat release also fluctuates. Additionally, velocity oscillations also induce heat release variations, which has been analysed in Section 7.3.3 for premixed flames.

In order to illustrate the acoustic effect on the motion of the droplets, an additional simulation is performed for $800 \mathrm{~Hz}$ with an inlet velocity amplitude of $80 \%$. The droplet distribution within the 1-D domain is shown in Figure 7.10, where the diameter of the droplets are plotted over their axial position. Droplets are injected at position $x=0$ into the domain and transported downstream towards the flame front. In the first two millimetres, droplet heating is recognized, as the droplet diameter increases due to the reduction of the liquid density. For higher axial positions the diameter decreases due to the evaporation. The impact of acoustic waves on the parcels' positions is identified by the formation of agglomerations. The regions where the high concentrated bands take 


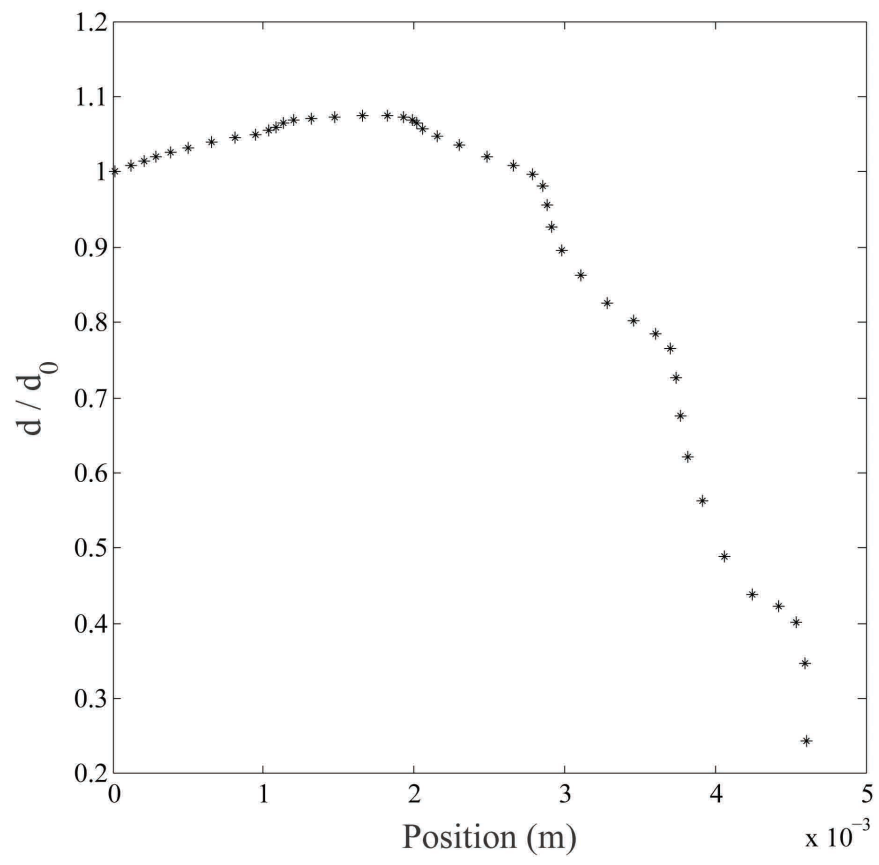

Figure 7.10: Position and non-dimensional droplet diameter of a 1-D spray flame under $800 \mathrm{~Hz}$ acoustic waves generated by inlet velocity oscillations with $80 \%$ amplitude.

place are followed by regions with low droplets concentration. This correlates with the shape of an acoustic wave. Hence, the droplet distribution depends on the perturbation's frequency and magnitude. This unsteady supply results in equivalence ratio fluctuations.
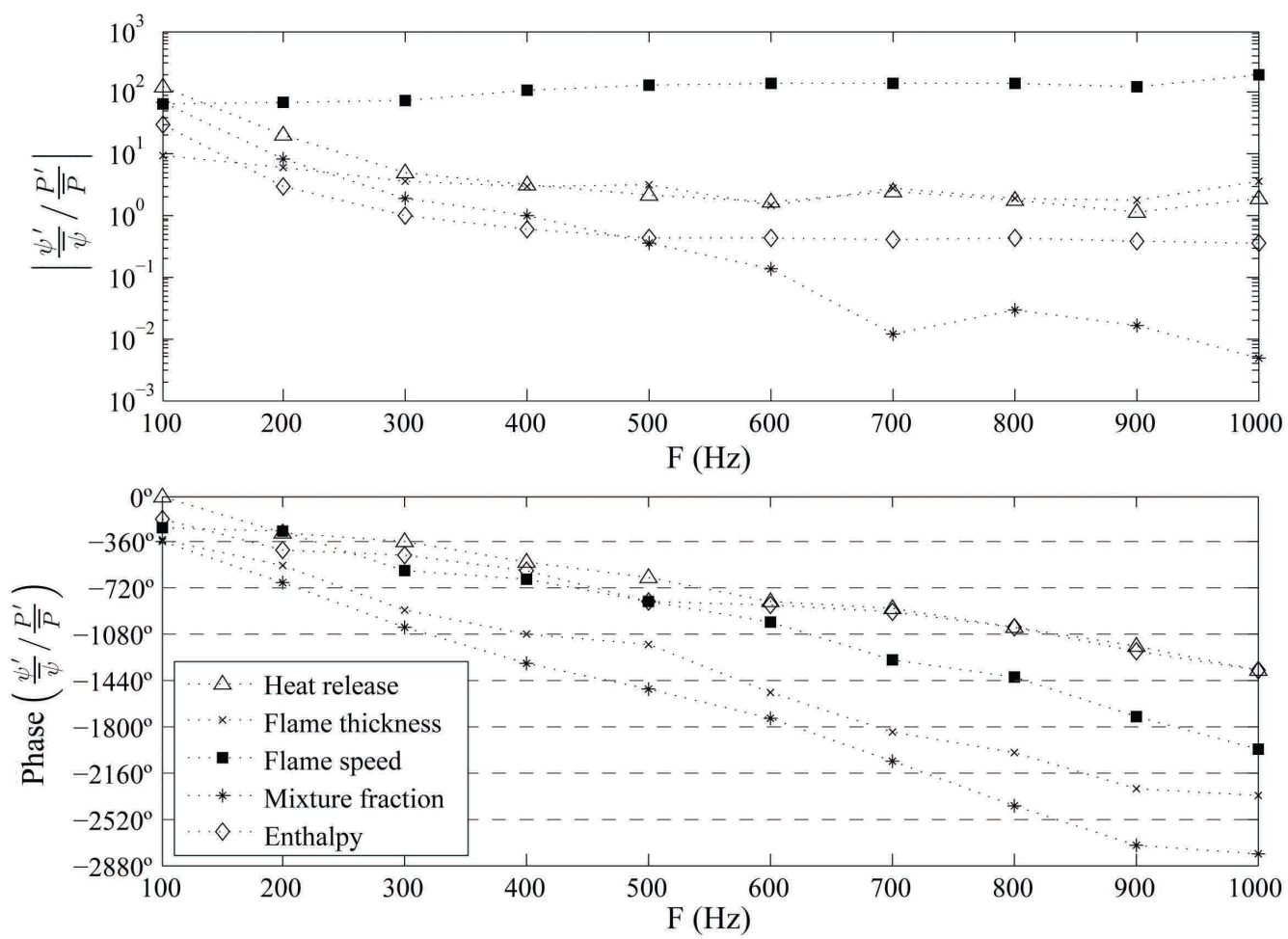

Figure 7.11: Bode diagram of the heat release, mixture enthalpy and fuel mass fraction transfer functions. 
The Bode diagram with the transfer functions of the monitored variables is depicted in Figure 7.11. Since most of them have a low-pass filter behaviour, a logarithmic scale is used for the vertical axis for a better visualization of the curves at high frequencies. As in the fully premixed combustion, the flame speed shows the highest response and the amplitude increases with the frequency. Conversely, the mixture fraction amplitude is maximum at $100 \mathrm{~Hz}$ and it decreases until a value lower than 0.01 at $1000 \mathrm{~Hz}$. The enthalpy amplitude also shows a low-pass filter behaviour, but in this case it is constant for the higher frequencies. The latter indicates that at low frequencies the enthalpy oscillations are amplified by the mixture fraction fluctuations. Based on this, the cut-off frequency for this variable is estimated to be around $500 \mathrm{~Hz}$. A similar effect is observed for both the volume-integrated heat release and the flame thickness, having the former a higher response at the lower frequencies. However, as in the 1-D combustion case, the amplitude at high frequencies is the same. The phase plot shows that the highest time lag corresponds to the mixture fraction, followed by the flame thickness and the flame speed. The lowest time lags are associated to the enthalpy and the heat release, which have a similar phase.

\subsubsection{Jet flame}

Simple configurations have been investigated in the previous sections, which did not have any geometrical area change.

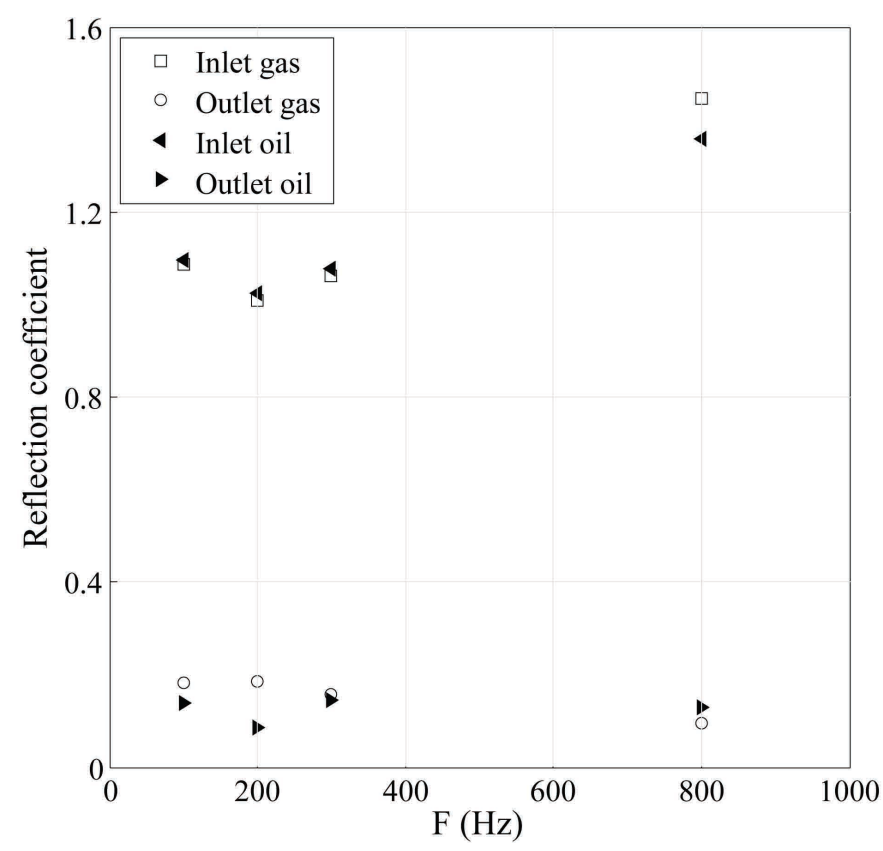

Figure 7.12: Reflection coefficients at the inlet and outlet boundaries for the oil and gas simulations.

The geometry of the Jet Flame case has are several area changes that partially reflect the acoustic waves. There is a large contraction from inlet plenum to the intake cone, 
which is followed by other area changes within the S-duct and an area expansion at the combustion chamber. Thus, prior to the calculation of the transfer functions, the multimicrophone technique is used to determine the reflection coefficient of the inlet and outlet boundaries. It is defined as the amplitude ratio between the waves entering and leaving the domain and it has been plotted in Figure 7.12 for each simulation. Small differences between the gas and oil simulations are observed. The waves that are generated by the inlet boundary condition are partially reflected at the intake cone and travel upstream. Thus, since the amplitude of the reflected wave (which leaves the domain) is smaller than the forcing amplitude, the reflection coefficient is larger than unity for the inlet boundary. Particularly, the larger values obtained for the $800 \mathrm{~Hz}$ simulations indicate that the reflection at the intake is a weaker than for the lower frequencies. At the outlet the reflection coefficient is between $10-20 \%$ for all the simulations. As aforementioned in Section 7.2 , a partial reflection is required to minimize the drift in the pressure level.

\section{Gas fuel}

Velocity an pressure time series at several points along the domain are monitored, as shown in Figure 7.13 for the $300 \mathrm{~Hz}$ simulation.

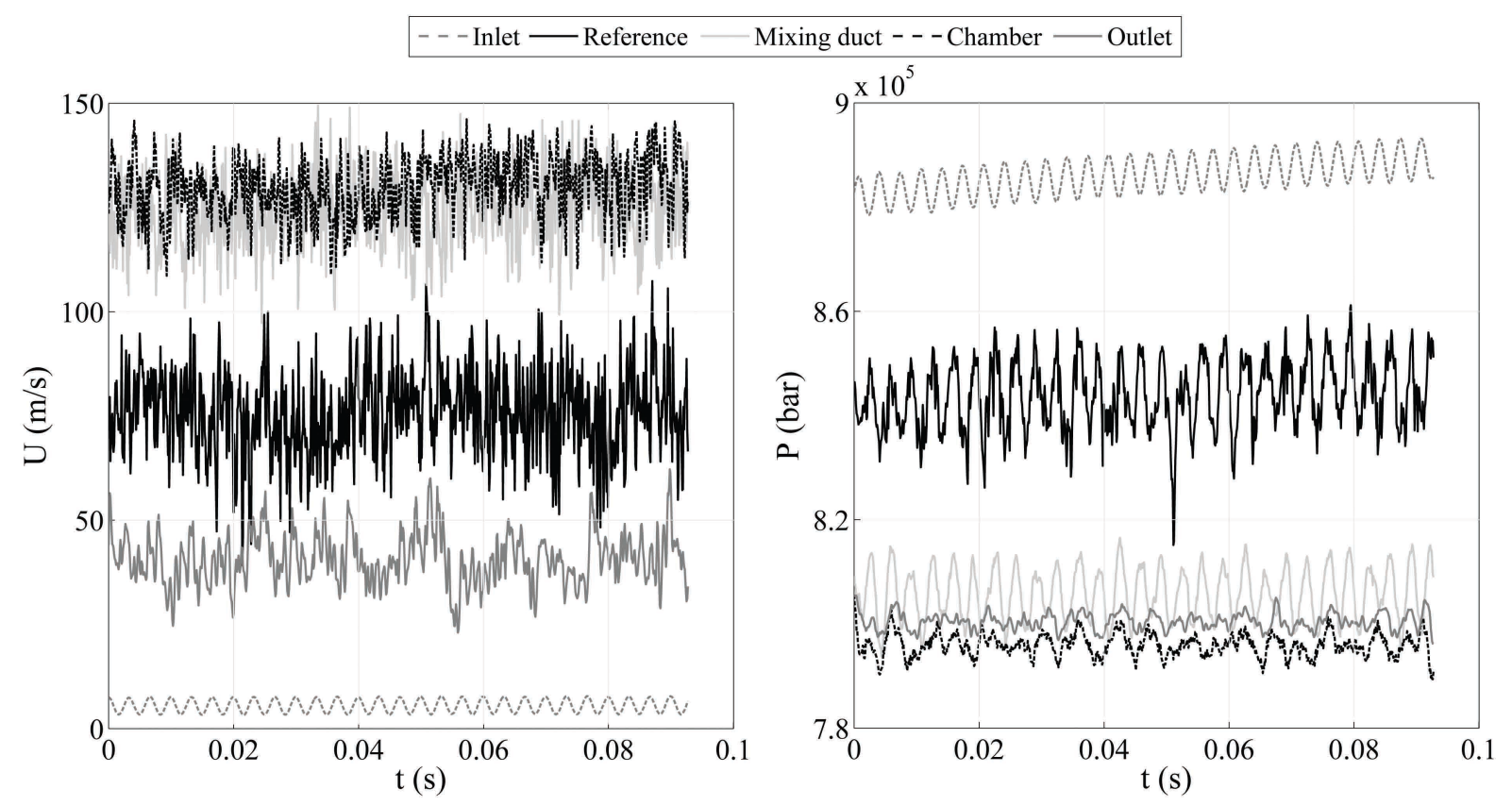

Figure 7.13: Velocity (left) and pressure (right) time series of the $300 \mathrm{~Hz}$ gas simulation that are sampled at the inlet boundary, upstream of the gas injection point, in the middle of the premixing duct, $100 \mathrm{~mm}$ downstream of the baseplate (aligned with the jet axis) and at outlet boundary.

The velocity at the inlet of the plenum has a sinusoidal shape. As the flow accelerates and passes through the S-duct, turbulence is generated so the signal at the reference point is noisy and the velocity waves cannot be clearly distinguished. The flow further 
accelerates within the mixing duct and at the chamber to decelerate downstream of the flame. Regarding the pressure, at the inlet location the mean value (over a oscillation period) increases with the simulation time. The latter implies that the mean global equivalence ratio decreases with time.

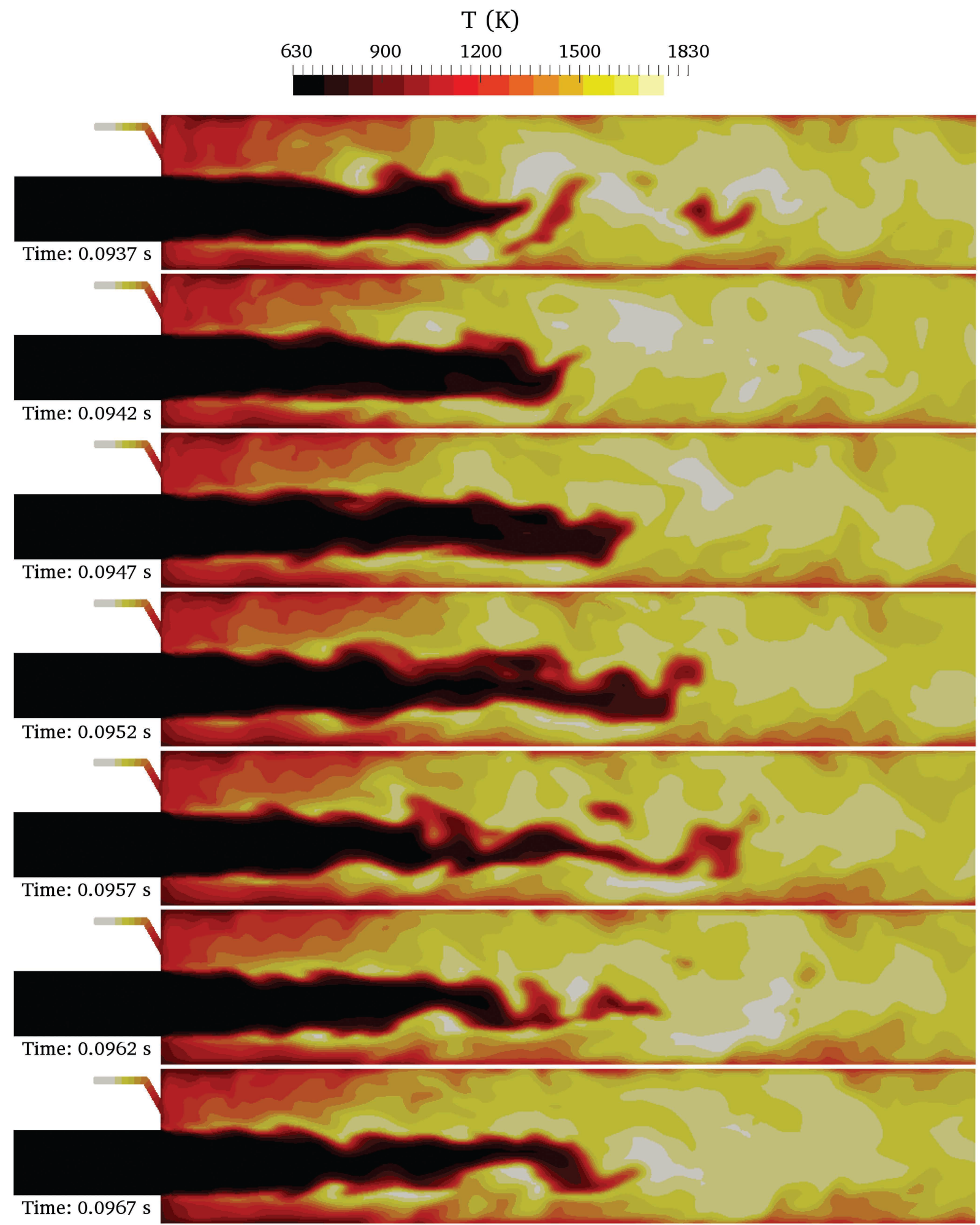

Figure 7.14: Temperature fields of the $300 \mathrm{~Hz}$ gas simulation at a mid-plane for several instants within an oscillation period. 
For consistency, simulations are performed so that the mean inlet pressure drift is the same in all cases (e.g. from 8.2 to 8.8 bar), including the ones with liquid fuel, and the relevant variables are post-processed over this period. This pressure drift is less evident in the other locations, especially those closer to the outlet. The fluctuations at the forcing frequency are more evident for this variable since it is less affected by turbulence, which is the reason to use it for the transfer functions, although the shape of the signals is not completely sinusoidal.

Several temperature contours over a oscillation period, which is about $3.3 \mathrm{~ms}$, are presented in Figure 7.14. The temperature downstream of the flame is not significantly affected by the acoustic waves, which indicates a low change of the mixture fraction for this frequency, and only the flame length and area oscillate. The amplitude of the flame length fluctuations is qualitatively similar for the other three frequencies considered. The change in the temperature distribution downstream of the flame due to mixture fraction oscillations is shown for the oil case in the next sub-section.
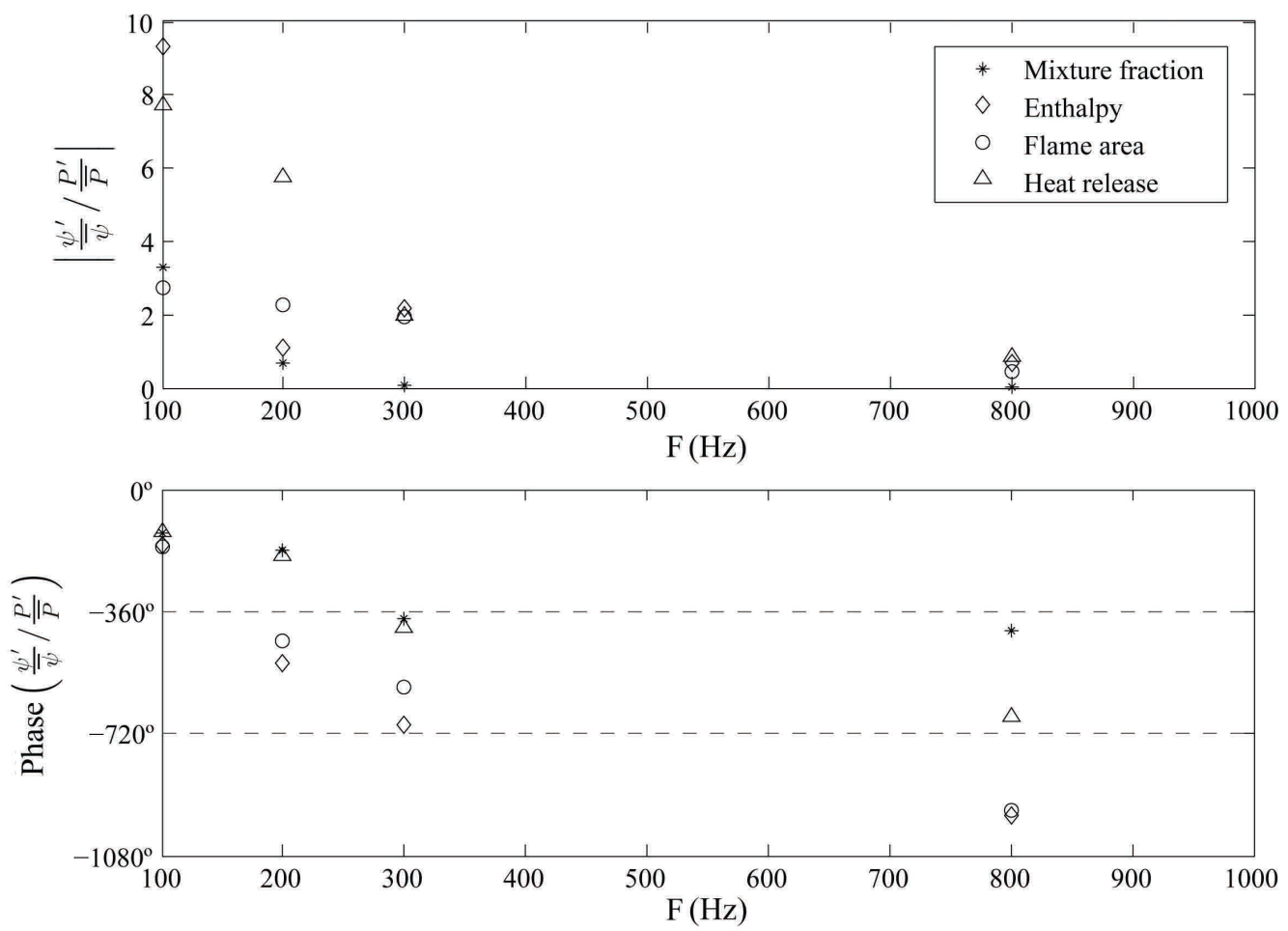

Figure 7.15: Bode diagram of the mixture fraction, the enthalpy, the flame area and the volume-integrated heat release transfer functions for the jet flame using methane as fuel.

The transfer function of the monitored variables is plotted on a Bode diagram in Figure 7.15. The amplitude of the mixture fraction is small for the 800 and $300 \mathrm{~Hz}$ and it increases progressively for the lower frequencies. In this configuration, unlike in the 1-D spray flame case, a larger response is obtained for the enthalpy even at the higher frequencies. The amplitude of the flame area decreases linearly with the frequency, while the one of the heat release presents a low-pass filter behaviour. For the lower frequencies, 
the phase plot shows that the heat release and the mixture fraction are in phase. The time lag of these variables is similar to the enthalpy time lag, despite the wrap-arounds, and larger than the one associated to the flame area. At $800 \mathrm{~Hz}$ the phases of the variables that have a significant amplitude are close to each other.

\section{Liquid fuel}

The velocity and pressure time series are also plotted for the $300 \mathrm{~Hz}$ oil simulation in Figure 7.16. Compared to the gas solution in Figure 7.13, the velocity is lower at both the premixing duct and combustion chamber locations while it is higher at the outlet. Additionally, the pressure level at the point placed within chamber is closer to the outlet pressure and both oscillate with a higher amplitude.
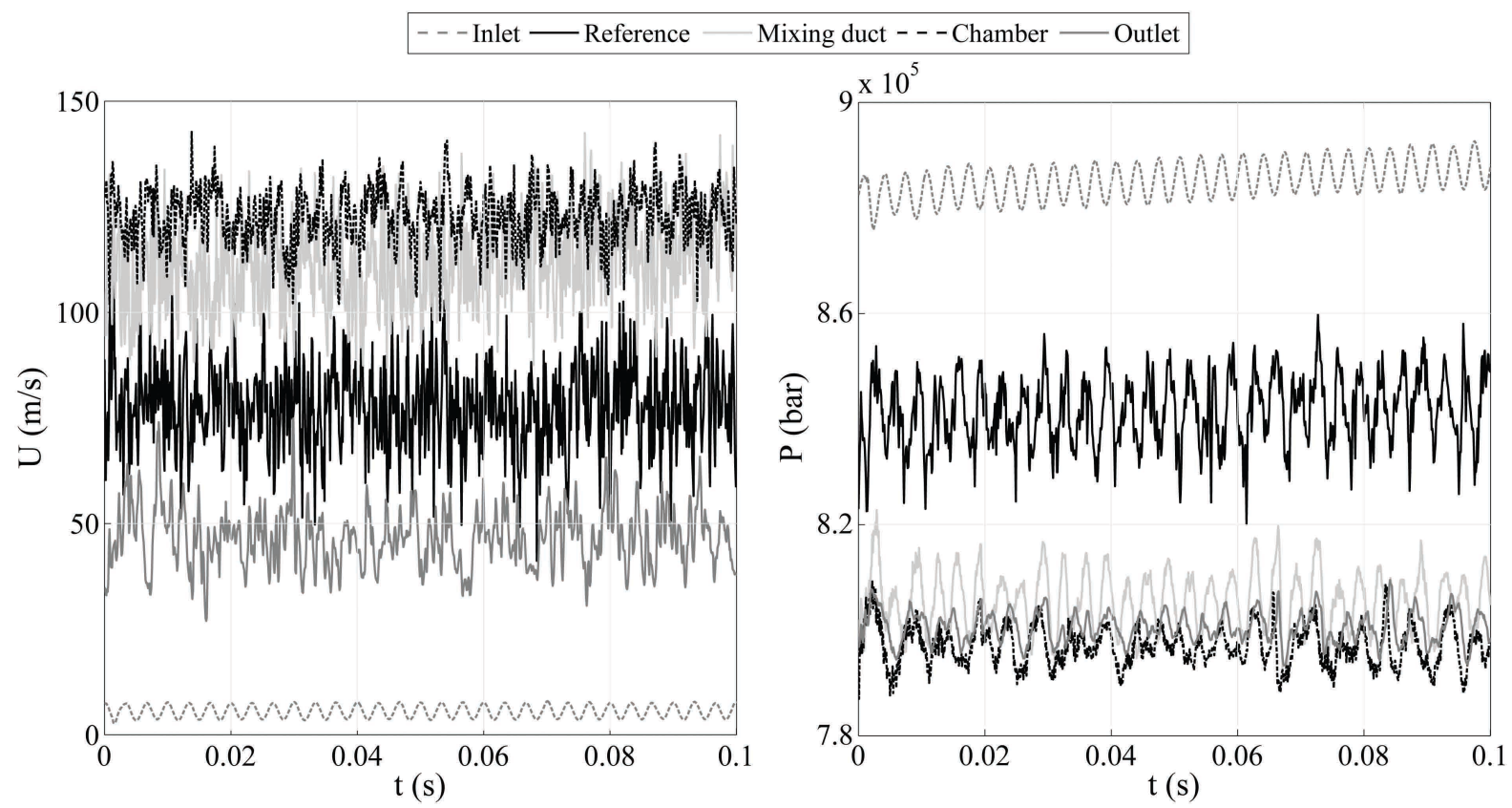

Figure 7.16: Velocity (left) and pressure (right) time series of the $300 \mathrm{~Hz}$ oil simulation that are sampled at the inlet boundary, upstream of the gas injection point, in the middle of the premixing duct, $100 \mathrm{~mm}$ downstream of the baseplate and at outlet boundary.

The transfer function of the liquid mass flow at the three sampling planes is plotted in Figure 7.17. It shows that the amplitude increases significantly with the axial distance, except for the for the $800 \mathrm{~Hz}$ simulation where the amplitude is similar. A low-pass filter behaviour can also be identified for this variable, especially for the $380 \mathrm{~mm}$ plane, due to the amplitude decrement from 100 to $300 \mathrm{~Hz}$. Similarly, the Bode diagram for the SMD is shown in Figure 7.17. At the first plane, the amplitude of this variable in the same order of magnitude as the liquid mass flow amplitude. However, the increase from the first to the second axial plane is smaller than with the liquid mass flow and it even decreases from the second to the last plane. The amplitude at $800 \mathrm{~Hz}$ is much lower than for the lower frequencies, which suggests a low-pass filter behaviour for this variable too. 

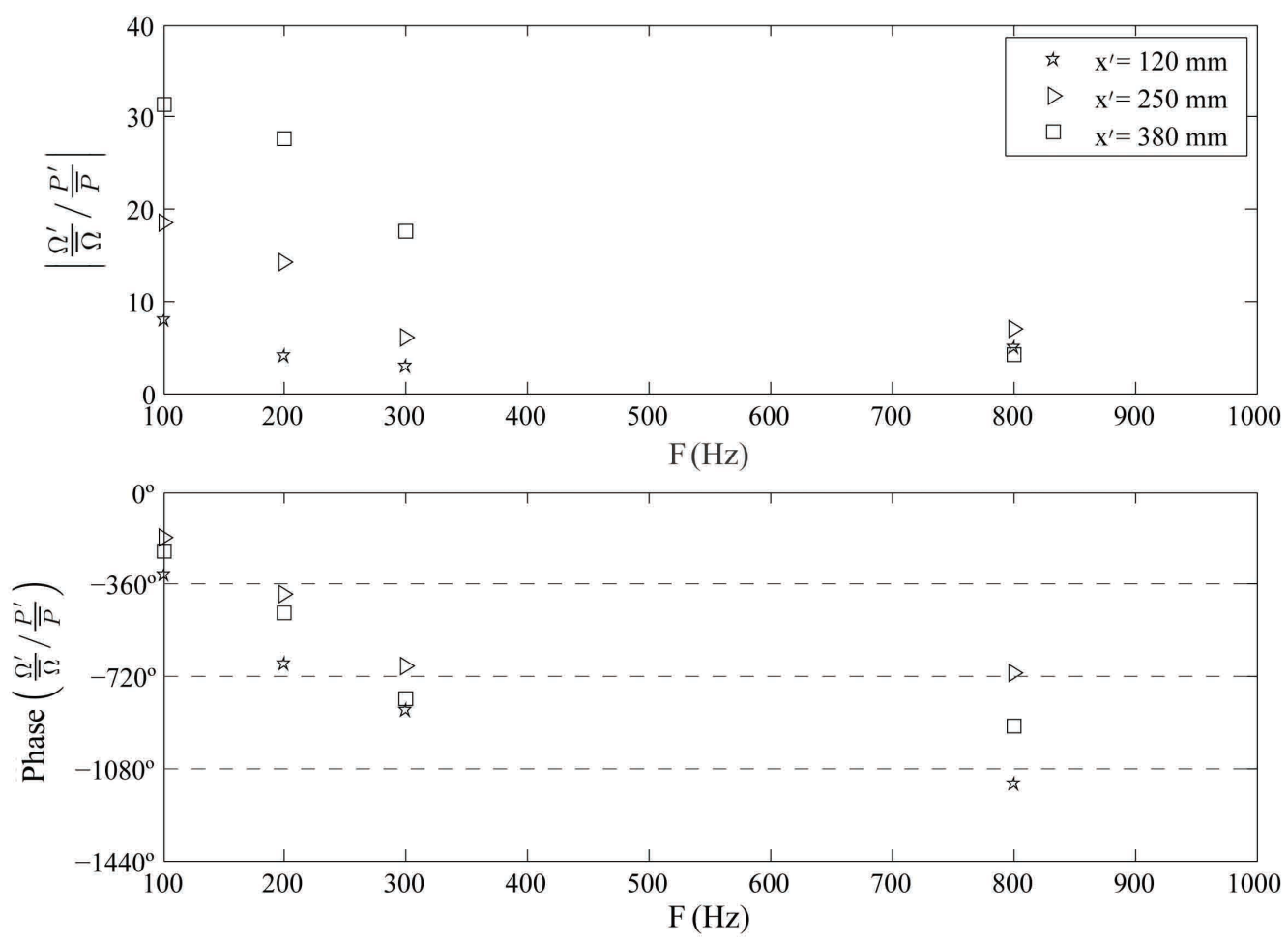

Figure 7.17: Bode diagram of the liquid mass flow transfer functions at three planes within the premixing duct for the jet flame using DF2 as fuel.
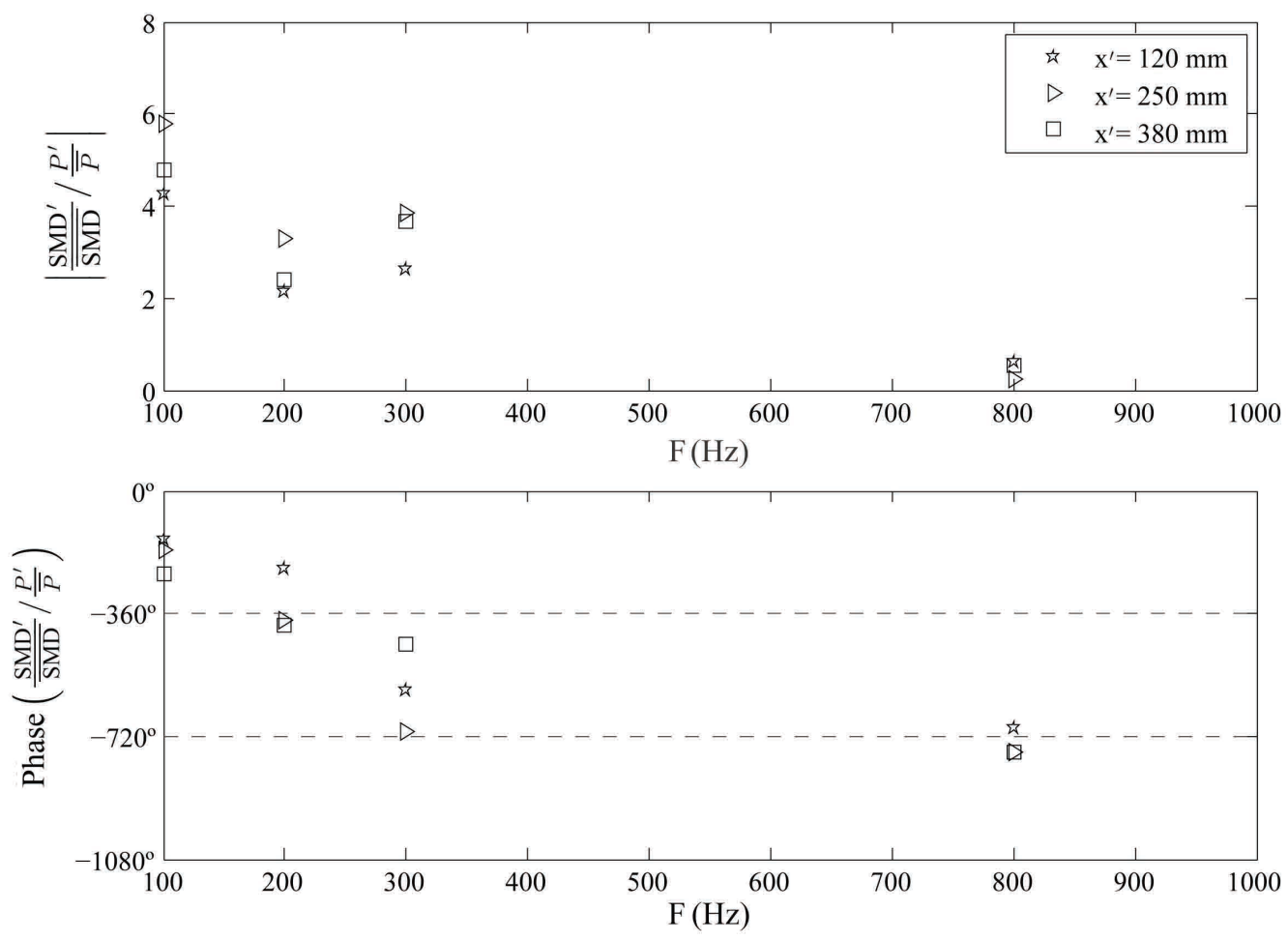

Figure 7.18: Bode diagram of the liquid mass flow transfer functions at three planes within the premixing duct for the jet flame using DF2 as fuel. 
The phase plots indicate that the time lag is lower for the SMD than for the liquid mass flow.

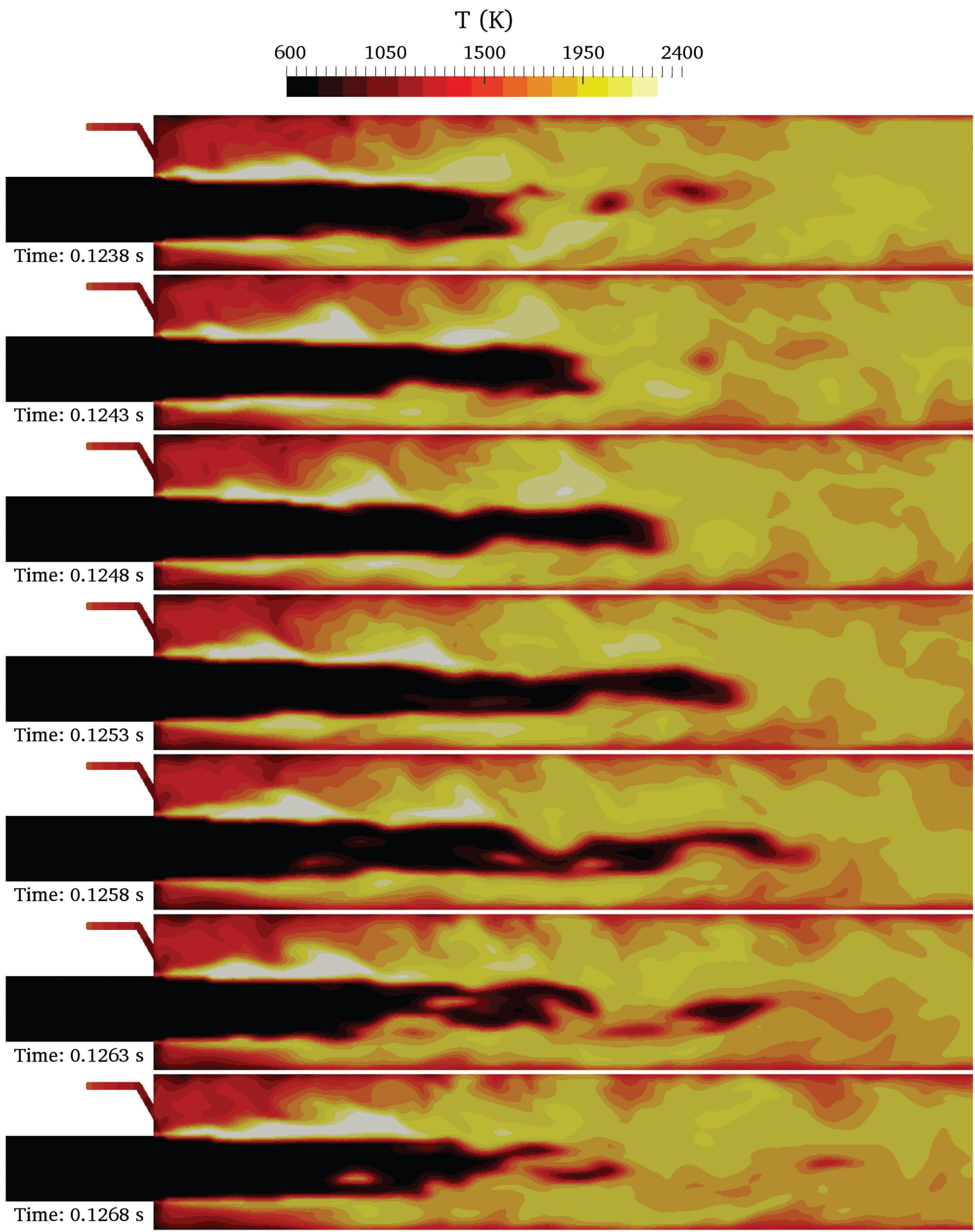

Figure 7.19: Temperature fields of the $300 \mathrm{~Hz}$ oil simulation at a mid-plane for several instants within an oscillation period.

The temperature contours for this simulation over a period are displayed above. Apart from the flame length oscillation, the temperature distribution downstream the flame (on 

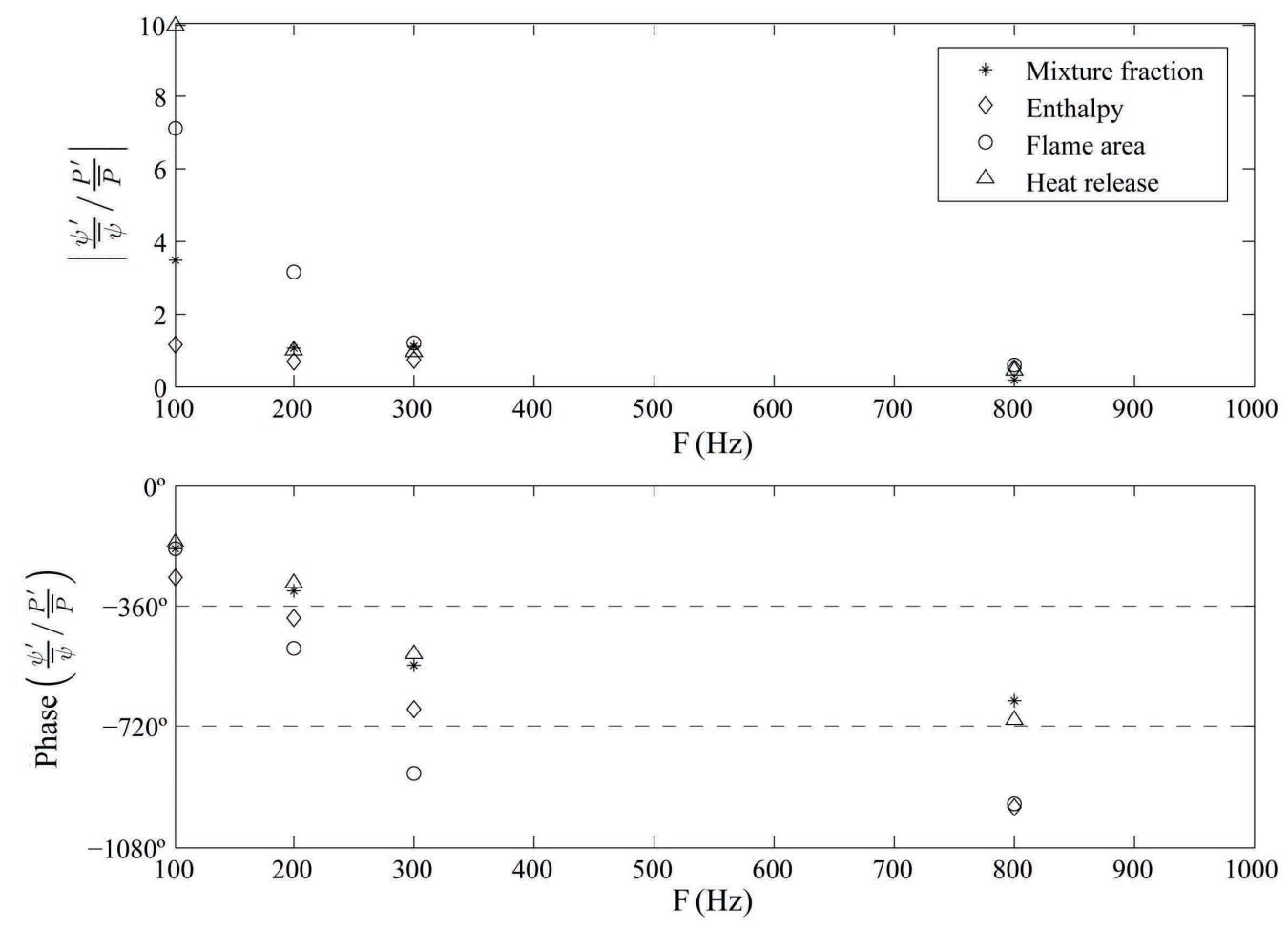

Figure 7.20: Bode diagram of the mixture fraction, the enthalpy, the flame area and the volume-integrated heat release transfer functions for the jet flame using DF2 as fuel.

the right side) also changes. On the first image the temperature is approximately $1950 \mathrm{~K}$ and in the three following pictures it becomes less uniform with larger regions that are 50$100 \mathrm{~K}$ lower. In the last three images the higher temperature regions become predominant again. The flame temperature predicted by the combustion model depends only on the mixture fraction and enthalpy variables, which are used in the look-up procedure. Since this effect was not visible with gas fuel and there were enthalpy fluctuations at the flame surface (see Figures 7.14 and 7.15 respectively), it is concluded that this effect is caused by mixture fraction oscillations. The transfer function of the mixture fraction, which is represented on a Bode diagram in Figure 7.20, confirms that the amplitude at $300 \mathrm{~Hz}$ is greater than in the gas fuel diagram in Figure 7.15. However, the amplitudes for the other frequencies are closer. The enthalpy amplitude remains almost constant for the simulated frequencies and with a lower value than for the gas fuel. In turn, the amplitudes of the flame area and heat release oscillations are larger for $100 \mathrm{~Hz}$ but the reduction with the frequency is steeper. The latter suggests that, for this test case, the cut-off frequency for the oil simulations is lower than for the gas ones. The phase plot shows that the mixture fraction and the volume-integrated heat release are in phase, as in Figure 7.15, having the enthalpy and the flame area a higher time lag as well. 


\section{References}

[1] H. J. Krediet. "Prediction of limit cycle pressure oscillations in gas turbine combustion systems using the flame describing function". PhD thesis. University of Twente, 2012.

[2] L. Tay Wo Chong et al. "Identification of Flame Transfer Functions Using LES of Turbulent Reacting Flows". In: ed. by Siegfried Wagner et al. Springer Berlin Heidelberg, 2010, pp. 255-266.

[3] T. Lieuwen and B. T. Zinn. "Theoretical Investigation of Combustion Instability Mechanisms in Lean Premixed Gas Turbines". In: 36th Aerospace Sciences Meeting and Exhibit. 1998.

[4] T. C. Lieuwen and V. Yang. Combustion Instabilities in Gas Turbine Engines: Operational Experience, Fundamental Mechanisms, and Modeling. Ed. by Progress in Astronautics and Aeronautics. AIAA, 2005.

[5] R. Kaess et al. "CFD-based mapping of the thermo-acoustic stability of a laminar premix burner". In: Proceedings of the Summer Program. 2008.

[6] A. Giauque, T. Poinsot and F. Nicoud. "Validation of a Flame Transfer Function Reconstruction Method for Complex Turbulent Configurations". In: 14th AIAA/CEAS Aeroacoustics Conference (29th AIAA Aeroacoustics Conference). 2008.

[7] M. Y. Ha. "A Numerical Study of Droplet Evaporation and Combustion in the Presence of an Oscillating Flow". In: Journal of Energy Resources Technology 119 (1997), pp. 109-119.

[8] T. J. Anderson et al. "Measurement of spray/acoustic coupling in gas turbine fuel injectors". In: 36th AIAA Aerospace Sciences Meeting and Exhibit. 1998.

[9] T. J. Anderson, W. Proscia and J. M. Cohen. "Modulation of a Liquid-Fuel Jet in an Unsteady Cross-Flow". In: Proceedings of ASME Turbo Expo. 2001.

[10] J. Song, C. Ramasubramanian and J. G. Lee. "Response of Liquid Jet to Modulated Crossflow". In: Proceedings of ASME Turbo Expo. 2013.

[11] G. E. Cossali and S. Tonini. "Oscillating droplet evaporation modelling for spray combustion simulation". In: Droplet Impact Phenomena and Spray Investigation. 2012.

[12] J. H. Moon, B. H. Kang and H. Kim. "Effects of Acoustic Resonance on Hydrodynamics and Evaporaton of a Pendant Liquid Droplet". In: Proceedings of IPACK2005-73111. 2005.

[13] M. Zhu, A. P. Dowling and K. N. C. Bray. "Combustion Oscillations in Burners with Fuel Spray Atomisers". In: International Gas Turbine and Aeroengine Congress Exhibition. 1999. 
[14] T. Poinsot and D. Veynante. Theoretical and Numerical Combustion. R. T. Edwards, 2005 .

[15] T. J. Poinsot and S. K Lelef. "Boundary conditions for direct simulations of compressible viscous flows". In: Journal of Computational Physics 101 (1992), pp. 104129.

[16] J. Biet et al. "Experimental and modeling study of the low-temperature oxidation of large alkanes". In: Energy \& Fuels 22 (2008), pp. 2258-2269.

[17] M. Rachner et al. "Modelling of the atomization of a plain liquid fuel jet in crossflow at gas turbine conditions". In: Aerospace Science and Technology 6 (2002), pp. 495506 .

[18] J. C. Beale and R. Reitz. "Modelling spray atomization with the Kevin-Helmholtz/RayleighTaylor hybrid model". In: Atomization and Spray 9 (1999), pp. 623-650. 


\section{Chapter 8}

\section{Conclusions}

This thesis focused on the development and coupling of primary atomization, evaporation and combustion models for reacting spray simulations.

A novel coupling model between the Euler and Lagrangian formulations, which interpolates the gas phase properties and distributes the source terms by means of SPH ghost particles, has been developed for LES spray simulations. It has been combined with a primary atomization model where droplets with a diameter similar or larger than the cell size are injected. After a calibration stage, model results show a significantly lower mesh dependency than with the classic Particle-In-Cell coupling model. This allows the use of fine grids, to resolve smaller turbulent scales, without being limited by the droplet-to-cell volume ratio. A good agreement with experimental data, in terms of spray trajectories as well as liquid flux and SMD distributions, is obtained for kerosene jet A-1 fuels at several operation conditions.

For the droplet evaporation process, the Lewis assumption results in a better prediction of the species' mass fractions at the droplet surface than the Raoult's law at high ambient pressure and temperature conditions. In the case of emulsified fuels, an effective diffusivity parameter is used to model the transfer of water mass from the droplet core to the surface shell layer so that it can vaporize. Distillation limit is achieved at a high diffusivity while a oil shell is formed faster for lower diffusivity values.

The tabulated chemistry combustion model is a combination of the FGM and SCRS combustion models, which leads to a reduced size of the tables while the accuracy is not significantly affected. 1-D laminar premixed flames for hexadecane-water-air mixtures at several equivalence ratios and at two different pressures are simulated. Results, in terms of main species mass fraction profiles, flame temperature and flame speed, are in good agreement with the detailed flame solution.

With the Lagrangian approach, the atomization and evaporation models are automatically coupled. Simulations of evaporating hexadecane sprays in a cross flow show that, for the same axial distance, a higher percentage of the injected fuel is vaporized for the lower momentum ratios due to the reduced spray effects. Water addition leads to a larger 
evaporation rate near the injection, because of the higher vapour pressure of water, but the heating-up and evaporation of the hexadecane gets delayed. The evaporation and combustion models are coupled by means of the LES governing equations. The interaction between them is captured in 1-D spray flames, being the flame position greatly influenced by the initial droplet size. The effect of $10 \%$ initial water content on the 1-D spray flames has also been quantified.

The models have been applied to simulate the combustion process in a pressurized jet combustor using different fuel types. For gas operation, an appropriate setup of the wall thermal condition and the flame-turbulence interaction model results in a similar flame length and lift as in the experiments. A good agreement is obtained in the velocity profiles within the chamber for the Baseline case, but more discrepancies arise when the pilot tubes are activated. The flame intensity change for an increment in the propane content from 0 to $40 \%$ in mass is well captured by the combustion model. For liquid fuel operation, spray models determine the amount of fuel and its distribution at the inlet of the combustion chamber. The duct length is not long enough for a complete spray vaporization, especially when water is added, so that flame-spray interaction occurs. Due to spray-wall interaction, a higher fuel concentration forms at the duct wall (closer to stoichiometric conditions) than at the centre. The latter results in a more stable flame than with gas operation and the highest temperatures take place at the sides of the jet. Water addition increases the amount of liquid fuel at the channel walls, slows down the evaporation rate and reduces both the flame stability and intensity. Mie scattering measurements indicate that a similar amount of liquid mass flow enters the combustion chamber in the Diesel and Preheated cases. The mass flow for the latter case is significantly higher in the simulations, which indicates that some spray model assumptions are not adequate. Particularly, a more suitable droplet-wall interaction is required due to the significant wall impingement within the premixing duct.

The forced response approach is used to analyse the acoustic response models of the models. The droplet break-up and evaporation processes are enhanced in reference to the non-exited solution. For a jet in cross-flow, the most sensitive variable at an axial plane downstream of the injection is the liquid flux, which oscillates due to droplet dynamics (inertia and drag). Thus, the spray penetration and the SMD fluctuate with a smaller amplitude. The response of an evaporating droplet is non-linear due to the dependency of the model on the relative velocity, which oscillates at double the forcing frequency. For a laminar premixed flame, the volume-integrated heat release oscillations are caused by the velocity fluctuations, which determine the consumed fuel mass flow. Mixture fraction oscillations, which are caused by changes in the liquid flux, result in an increased amplitude of the heat release response. 


\section{Chapter 9}

\section{Recommendations}

The results of the jet flame simulations with liquid fuels, in Section 6.4.2, indicate that further modelling effort is necessary. In particular, the following aspects should be addressed in future work:

1. Implement an evaporating multi-component film model for the droplet-wall interaction, which determines whether a droplet is added to the film or rebounds - with or without breakup - based on the wall temperature and the impact conditions.

2. Implement a sub-grid spray dispersion model for LES.

3. Further validate the atomization - for several injection angles - and evaporation models for diesel fuel, with and without water addition.

4. Consider a non-uniform temperature distribution within the big droplets, at least for model validation.

5. Extend the heat transfer and evaporation models to account for the shape of the jet and primary droplets.

6. Evaluate the radiation model from the standard OpenFOAM version and analyse its effect on the spray evaporation.

7. Simulate the jet flames with a more adequate fuel discretization.

8. Investigate the reason for discrepancies of the jet flame simulations with liquid fuels if they are still present with the above developments.

9. Depending on the additional computational cost, consider the use of a dynamic mesh refinement method around the flame front so that the ATF model is not needed.

10. Perform the simulations with higher order spatial and temporal schemes.

11. Improve the parallel efficiency of the spray simulations: 
- Develop a decomposition method that, after an initial simulation, considers the location of the parcels to create the mesh partitions. Particularly, the amount of processors that contain primary and jet parcels should be minimized so that few virtual SPH particles are required.

- After the interpolation of the gas phase properties, uniformly re-arrange the parcels between the processors to evolve the models and pass them back to the original processor to distribute the source terms.

12. Extend the combustion model so that it is able to predict $\mathrm{CO}$ and NOx emission levels.

13. Analyse the linear acoustic behaviour of the models by imposing different forcing amplitudes.

14. Use the same inlet pressure amplitude in the acoustic study instead of the same inlet velocity amplitude.

15. Simulate the response of the jet flame with $400-700 \mathrm{~Hz}$ forcing frequencies.

16. Perform the jet flame acoustic simulations with a finer mesh to resolve higher turbulent scales, with a lower time step to reduce the acoustic CFL number and allowing the flame to lift from the baseplate.

17. Consider other post-processing variables, like the volume-integrated species source terms, or the location where these variables are area-averaged (e.g. at the end of the premixing duct) in the acoustic simulations.

18. Carry out an acoustic analysis of the models with water addition. 


\section{Appendix A}

\section{Atomization of a liquid jet at 40 degree injection angle}

The primary atomization model, presented in Section 2.2.1, has been validated in Section 2.3.3 for kerosene fuel injected at $90^{\circ}$ injection angles from the cross flow direction. However, as mentioned in Section 6.3, diesel fuel is injected at $40^{\circ}$ angle from the axial direction in the jet combustor test case. Thus, prior to the liquid fuel simulations shown in Section 6.4.2 the atomization model has been adjusted. For this, experiments with diesel fuel injection into a nitrogen cross flow at $40^{\circ}$ angle were internally performed at Siemens by B. Werdelmann [1]. The channel section is a $60 \times 60 \mathrm{~mm}^{2}$ square while the channel length is $400 \mathrm{~mm}$.

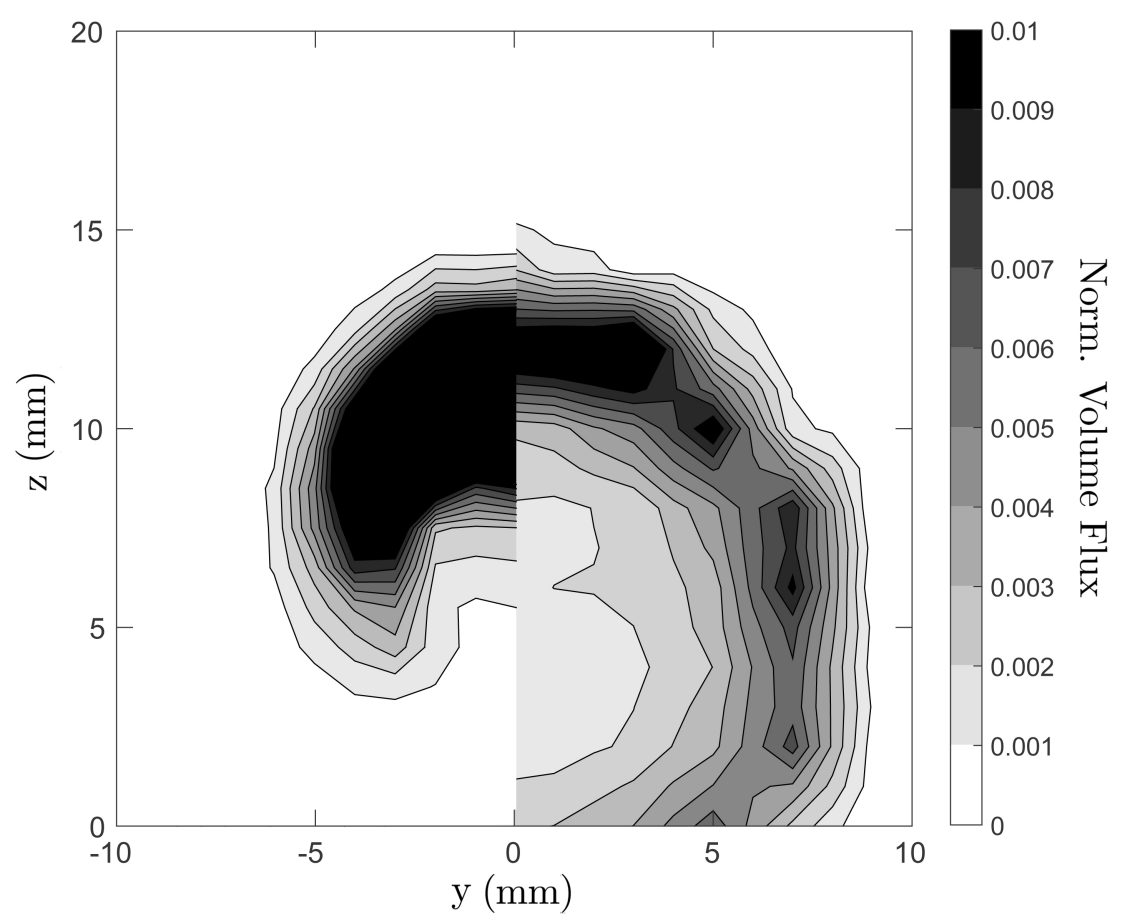

Figure A.1: Comparison of the time-averaged normalized volume flux distribution between experimental data from Werdelmann [1] (right) and simulation results (left). 


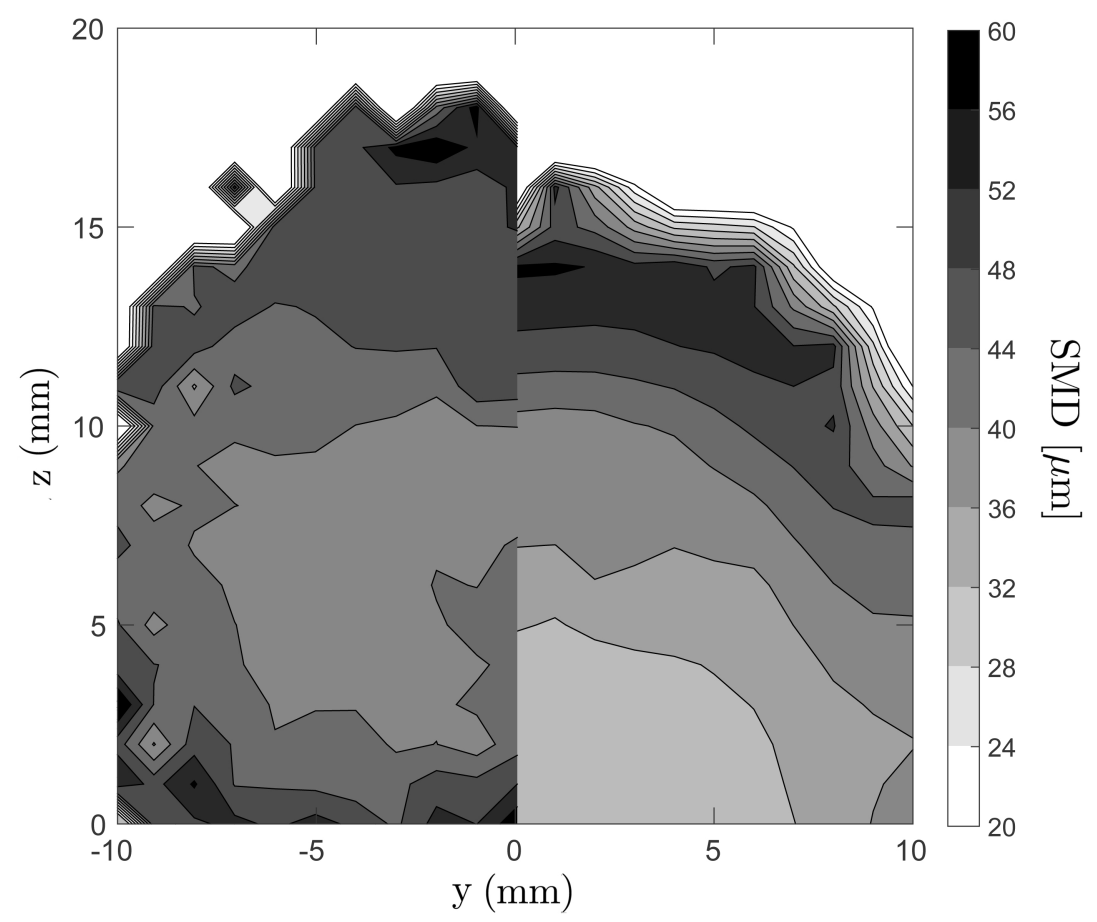

Figure A.2: Comparison of the time-averaged SMD distribution between experimental data from Werdelmann [1] (right) and simulation results (left)

The same meshing approach as in Figure 2.3, with two refinement levels around the injection point, is applied. The upstream velocity is about $100 \mathrm{~m} / \mathrm{s}$ and the pressure level is 8.5 bar. The injector hole diameter is $0.5 \mathrm{~mm}$ and the momentum ratio is 20, which is calculated using the liquid velocity component perpendicular to the cross flow in Equation (2.5). For this case, no turbulence generator is used since the inlet boundary is located at a plenum upstream of the channel.

Both the SMD and the volume flux distributions were measured experimentally at a plane $70 \mathrm{~mm}$ downstream of the injection point. The comparison of the flux with the simulation results is shown in Figure A.1. In order to match the penetration depth, the $C_{c o l}$ parameter from Equation (2.11) has been lowered from 2.8 to 1. The latter implies that the column break up occurs earlier than for $90^{\circ}$ injection angles. The simulations show that the shape of the spray is similar to the one in the experiments. However, the spray dispersion is significantly under-predicted, especially at the sides and at the bottom of the channel. The use of a turbulence generator or a sub-grid dispersion model could decrease these discrepancies. The SMD level is in good agreement with the experimental data at heights over $7 \mathrm{~mm}$. Near the bottom side, where there is no significant flux, the primary atomization model tends to over-predict the SMD.

\section{References}

[1] B. Werdelmann. Inclined liquid jets in cross flows. Tech. rep. Siemens AG, 2015. 


\section{Appendix B}

\section{Evaporation of mono-component droplets under natural and forced convection}

In Section 3.3, the evaporation model results for bi-component droplets under mixed convection conditions (i.e. an initial period with natural convection followed by a forced convection period) has been compared to the experimental data from Daif et al. [1]. As a previous step, the evaporation of mono-component droplets under either natural or forced convection is simulated.

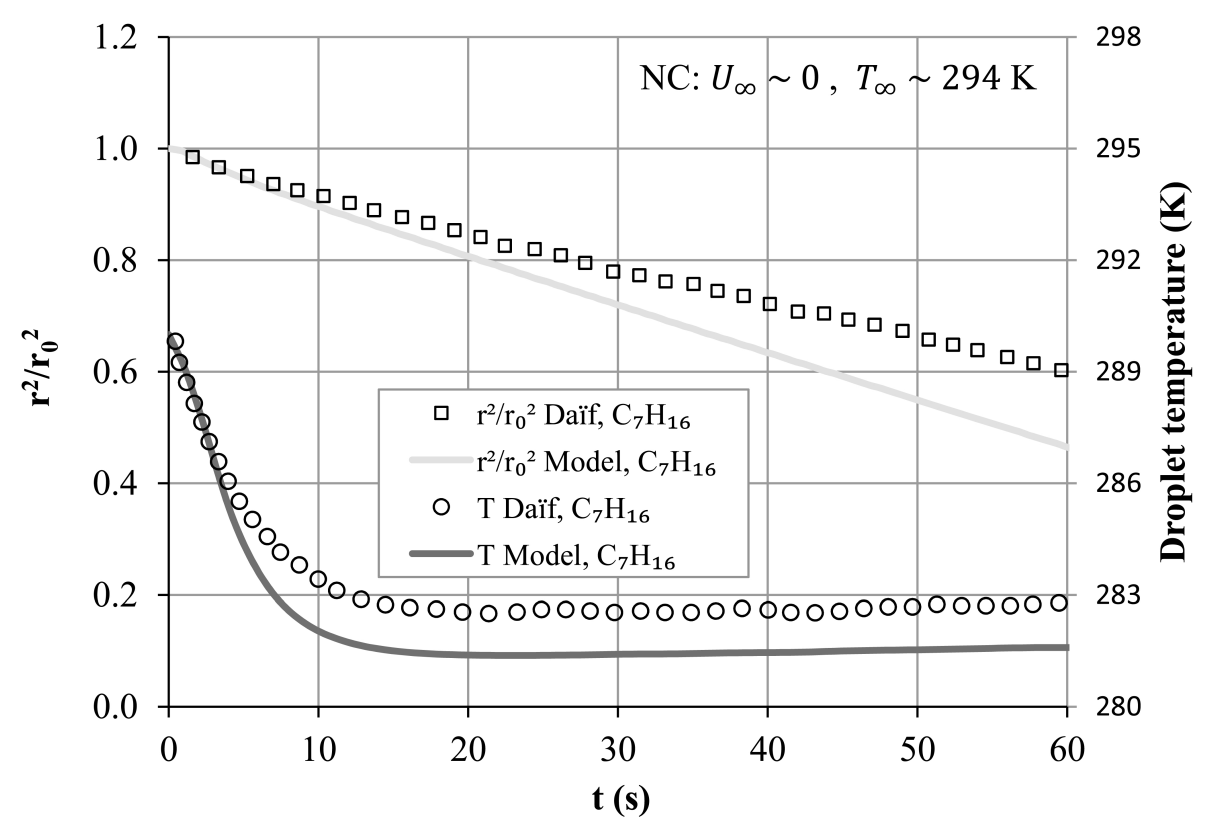

Figure B.1: Model results for the evaporation of a n-heptane under natural convection compared against experimental data from Daif et al. [1].

Both the temperature and diameter evolution of a n-heptane droplet under natural convection is shown in Figure B.1. In this case the ambient temperature is $4 \mathrm{~K}$ above the 
initial droplet temperature. The amount of evaporated mass is mainly determined by the vapour pressure. Since the heat transferred from the gas phase is not enough to maintain this mass flow, the latent heat is absorbed from the droplet and therefore the temperature decreases. The determination of the vapour pressure at these temperatures in not very precise and this leads to a significant deviation of the diameter curve. Particularly, the evaporated mass is over-predicted and this causes a lower equilibrium droplet temperature than in the experiments.

Results for the forced convection simulations are plotted in Figure B.2. The diameter curves are in good agreement with the experimental results. However, a higher droplet heat-up and equilibrium droplet temperature is obtained for the n-decane droplet. This indicates that the heat transferred from the gas phase is over-predicted and leads to a slightly higher evaporation rate.

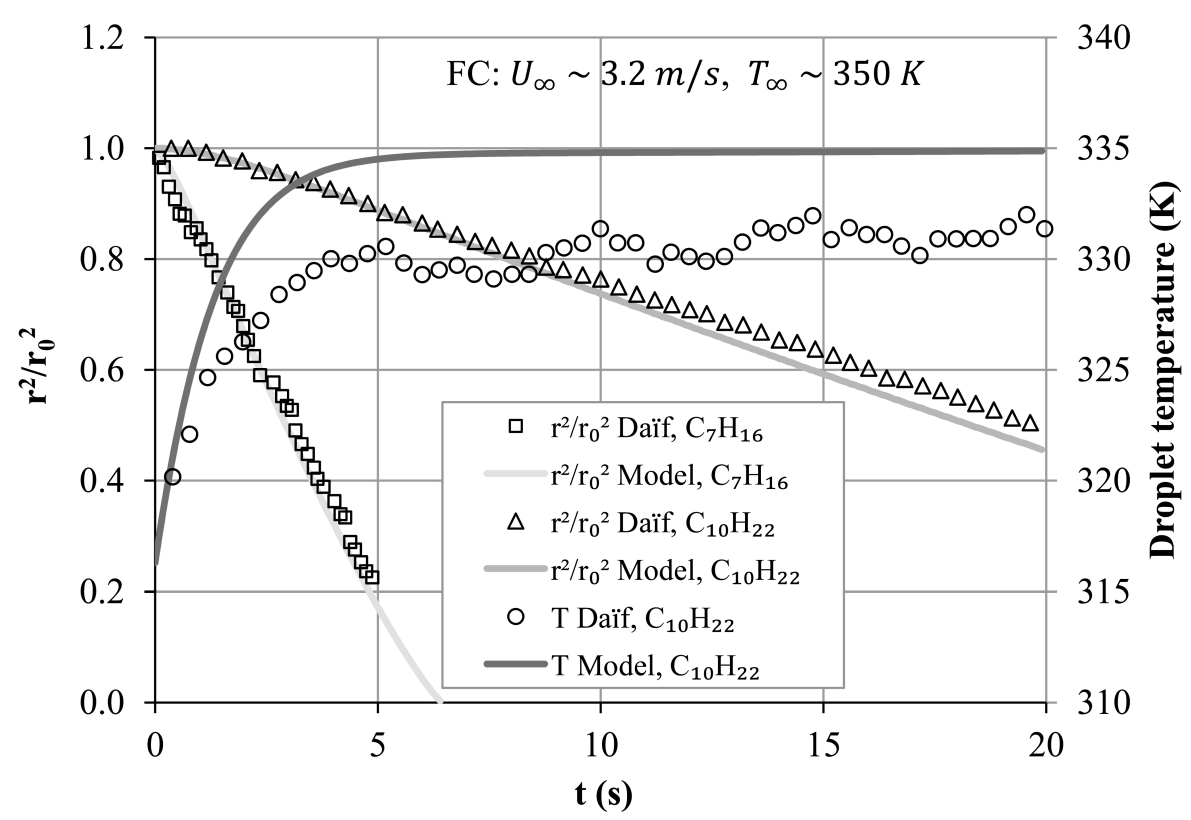

Figure B.2: Model results for the evaporation of a n-decane and n-heptane droplet natural convection compared against experimental data from Daïf et al. [1].

\section{Mesh sensitivity}

The impact of the cell size on the life time of the n-heptane droplet under forced convection, which has been simulated in Figure B.2, is investigated. The same box dimensions as in the previous simulations is used and the cell size is varied from 1.5 to $6 \mathrm{~mm}$. Simulations are performed with both the PIC and GPIC coupling models for mass, momentum, energy and species transfer. The droplet is initialized with a diameter of $d_{0}=1.052 \mathrm{~mm}$ at the center of a cell close to the middle of the box. This consideration is important for the comparison between the PIC and GPIC results, because if the droplet is initialized at one face or at a vertex the ghosts parcels are distributed to a different number of cells in 


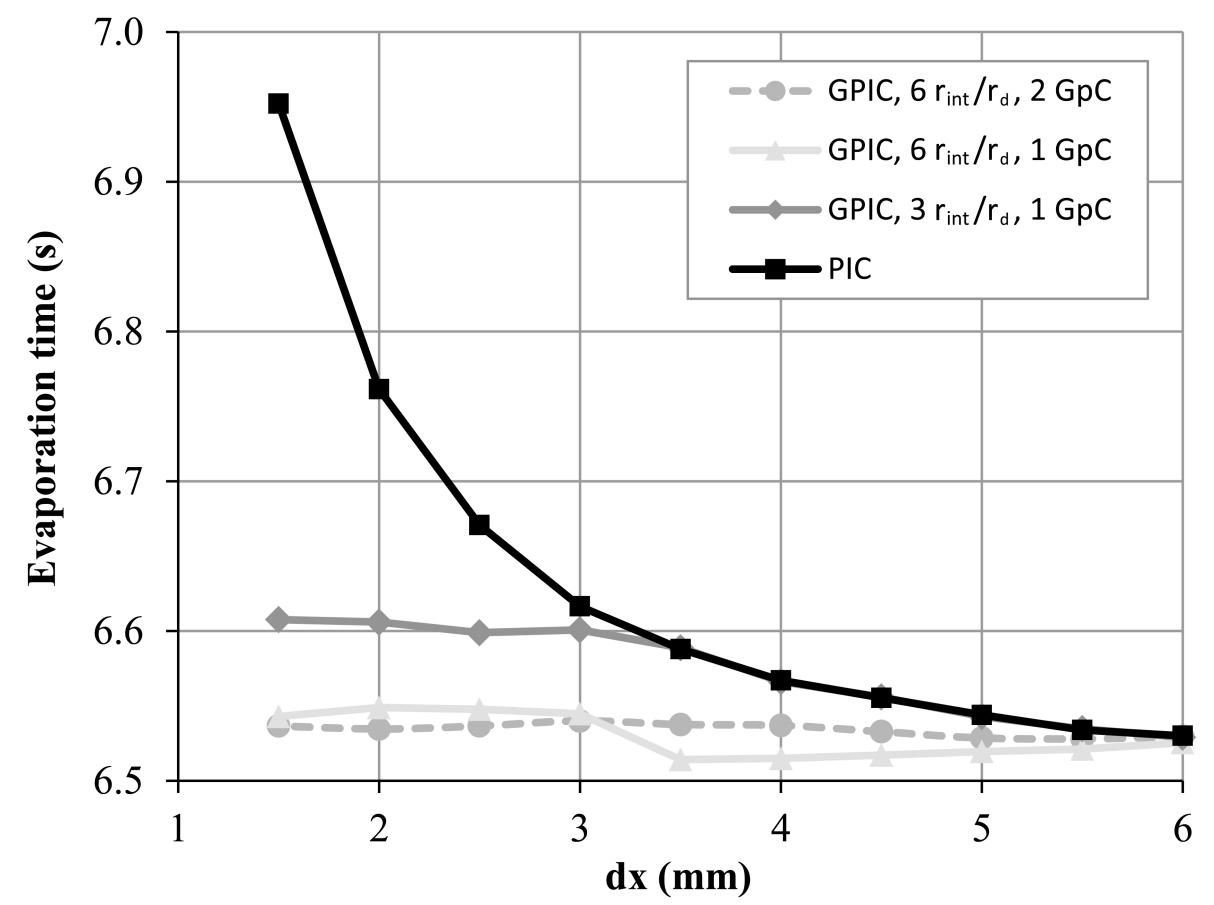

Figure B.3: Effect of the cell size on the evaporation time of an n-heptane droplet under forced convection, with $U_{\infty}=3.2 \mathrm{~m} / \mathrm{s}, d_{0}=1.052 \mathrm{~mm}$ and $T_{\infty}=356 \mathrm{~K}$, using both the PIC and GPIC models.

each case. Particularly, if the droplet is always initialized at the box centre, the solution with the GPIC model would strongly depend on whether an odd (cell centre) or even (cell vertex) number of cells per direction are used.

Simulation results are plotted in Figure B.3. For the PIC model, the evaporation time increases exponentially as the cell size is reduced. For the GPIC model, three configurations are simulated:

1. One ghost particle per cell and an interaction distance three times the droplet radius.

2. One ghost particle per cell and an interaction distance six times the droplet radius.

3. Two ghost particles per cell and an interaction distance six times the droplet radius.

In the first configuration, the same solution as the PIC model is obtained when $r_{\text {int }}>$ $d x / 2$. Since the initial diameter is slightly above $1 \mathrm{~mm}$, for $d x=3 \mathrm{~mm}$ the source terms are also distributed to the neighbouring cells until the diameter falls below $1 \mathrm{~mm}$. In this period, the source term is transferred to a larger volume and therefore a shorter evaporation time is obtained. For lower cell sizes, the evaporation time is similar to the one obtained at $d x=3 \mathrm{~mm}$ (and theoretically at $d x=2 r_{\text {int }}$ ).

With a double interaction radius, the source term starts to be distributed from $d x=$ $6 \mathrm{~mm}$ since the evaporation time is slightly lower to the value obtained with the PIC model. This is caused by the source term transfer to a higher volume. As the cell size is 
reduced, the time period when the source term gets distributed is higher. The latter leads to a shorter droplet life time. The trend changes at $d x=3 \mathrm{~mm}$, where there is a jump in the evaporation time from $d x=3.5 \mathrm{~mm}$. This occurs because the cell size has reached a sub-multiple of the interaction radius, in this case half of it, so that the source term is transferred to a similar volume as with $d x=6 \mathrm{~mm}$. For the lower cell sizes, the droplet life time remains close to the value with $d x=3 \mathrm{~mm}$. The increase to two ghost particles per cell effectively removes the jump observed in the second configuration because the source term weight of the cell that contains the droplet increases. Thus, a low mesh dependency is obtained with the third model setup.

\section{References}

[1] A. Daï, M. Bouaziz and A. A. Chérif. "Comparison of multicomponent fuel droplet vaporization experiments in forced convection with the Sirignano model". In: Experimental Thermal and Fluid Science 18.4 (1999), pp. 282-290. 


\section{Appendix C}

\section{Evaporation of heptane droplets under micro-gravity}

The evaporation model has been evaluated in Section 3.3 and in Appendix B for droplets under both natural or forced convection.
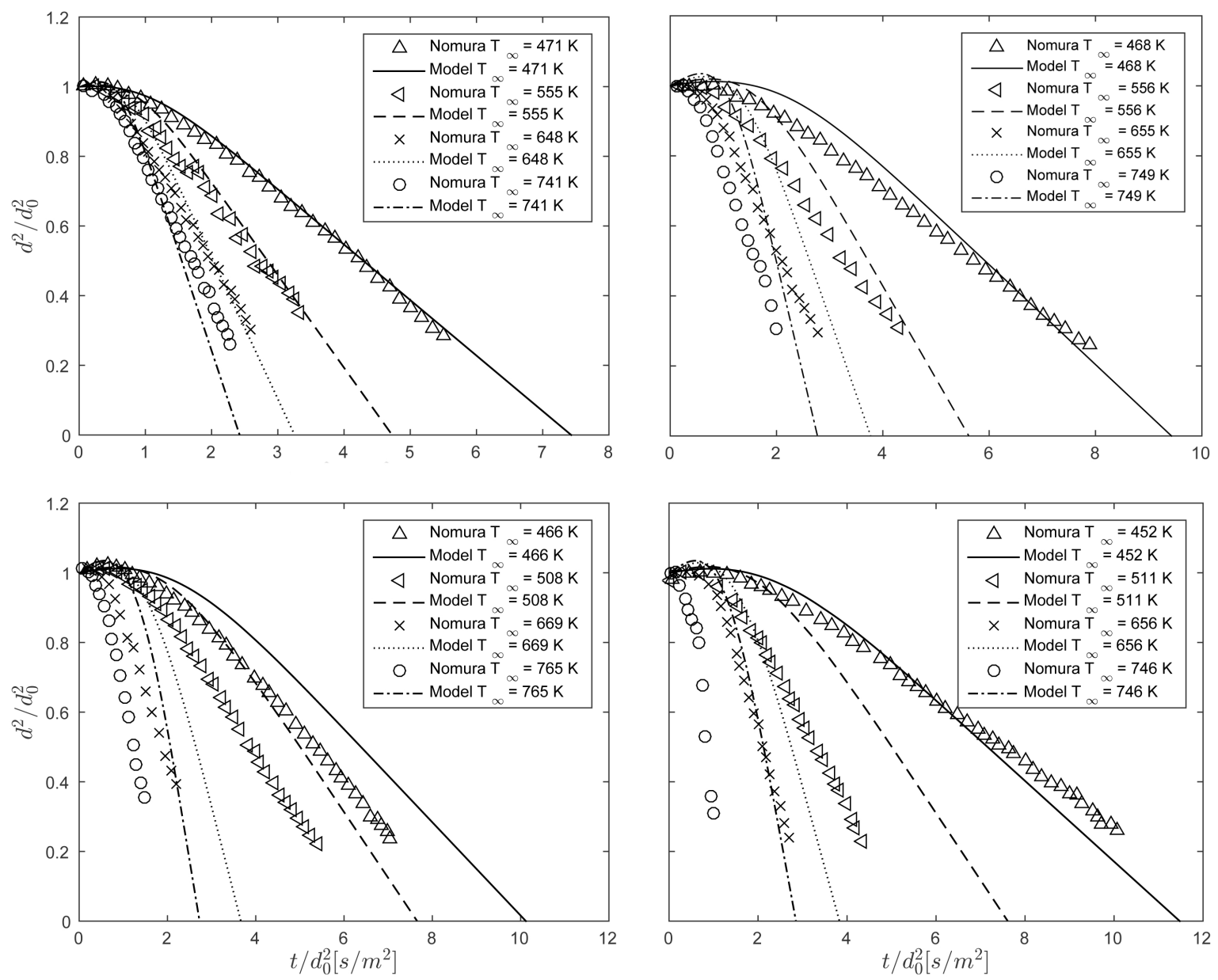

Figure C.1: Model results of the droplet diameter evolution for n-heptane droplets under micro-gravity compared against experimental data from Nomura et al. [1]. Pressures are 1 bar (top left), 5 bar (top right), 10 bar (bottom left) and 20 bar (bottom right). 
In this section, the evaporation of n-heptane droplets under micro-gravity conditions are simulated. The same setup used in Section 3.3 is applied. The evolution of the droplet diameter, non-dimensionalized with the initial diameter, is compared to experimental data from Nomura [1] in Figure C.1 for several ambient pressures and temperatures. A good agreement is is obtained for atmospheric conditions, especially at low ambient temperatures, in terms of evaporation rate and droplet life time. At 5 bar, despite the life time is well predicted, a higher heating peak and evaporation rate are over-predicted by the model. For the 10 and 20 bar cases, the discrepancies in the diameter peak increase while the slope of the curves in the steady evaporation region are more similar to the measured ones.

\section{References}

[1] H. Nomura et al. "Experimental study on high-pressure droplet evaporation using microgravity conditions". In: Twenty Sixth Symposium (International) on Combustion. 1996. 


\section{Appendix D}

\section{Combustion model formulation for bi-component fuels}

The formulation exposed in Section 4.2 is valid only for a hydrocarbon fuel mixed with a inert specie, whose mass fraction is not consumed within the flame, like water vapour or any inert gas. In this section, the model is extended to consider bi-component hydrocarbon species that are both consumed at the flame front.

\section{Calculation procedure}

The one-step global reaction for the combustion of two hydrocarbons is:

$$
\begin{gathered}
C_{n} H_{m}+\nu C_{p} H_{q}+\frac{\left(n+\frac{m}{4}\right)+\nu\left(p+\frac{q}{4}\right)}{\phi} O_{2}+\frac{\left(n+\frac{m}{4}\right)+\nu\left(p+\frac{q}{4}\right)}{\phi} \frac{1-b}{b} N_{2} \\
\rightarrow \epsilon \mathrm{CO}_{2}+\beta C O+\eta \mathrm{H}_{2} \mathrm{O}+\theta \mathrm{H}_{2}+\zeta \mathrm{O}_{2}+\frac{\left(n+\frac{m}{4}\right)+\nu\left(p+\frac{q}{4}\right)}{\phi} \frac{1-b}{b} N_{2}
\end{gathered}
$$

The equivalence ratio is calculated as following:

$$
\phi=A F R_{s t_{C_{n} H_{m}}} \frac{Z(1-W)}{1-Z}\left(1+\frac{W}{1-W} \frac{M_{C_{n} H_{m}}}{M_{C_{p} H_{q}}} \frac{p+\frac{q}{4}}{n+\frac{m}{4}}\right)
$$

where:

$$
A F R_{s t_{C_{n} H_{m}}}=\left(n+\frac{m}{4}\right) \frac{M_{O_{2}}+\frac{1-b}{b} M_{N_{2}}}{M_{C_{n} H_{m}}}
$$

The calculation of the $N_{2}$ mass fraction is computed directly from $Z$ and $W$ :

$$
Y_{N_{2}}=M_{N_{2}} \frac{Z(1-W)}{M_{C_{n} H_{m}}} \frac{1-b}{b} \frac{\left(n+\frac{m}{4}\right)+\nu\left(p+\frac{q}{4}\right)}{\phi}
$$


The mass fractions for both hydrocarbons are obtained from the progress variable:

$$
\begin{gathered}
Y_{C_{n} H_{m}}=Z(1-W)(1-c) \\
Y_{C_{p} H_{q}}=Z-Y_{C_{n} H_{m}}
\end{gathered}
$$

Following this, the mixture fraction of $C O$ is obtained from the conservation of carbon atoms:

$$
Y_{C O}=M_{C O}\left(\frac{n(1-W)}{M_{C_{n} H_{m}}}+\frac{p W}{M_{C_{p} H_{q}}}\right)\left(Z-Y_{C_{n} H_{m}}-Y_{C_{p} H_{q}}\right)-\frac{M_{C O}}{M_{C O_{2}}} Y_{C O_{2}}
$$

For equivalence ratios $\phi<1.2$, the mass fraction of water is obtained from the conservation of hydrogen atoms assuming a zero mass fraction of the hydrogen molecule:

$$
\begin{aligned}
& Y_{H_{2} O}=\frac{M_{H_{2} O}}{2}\left\{\left(\frac{m(1-W)}{M_{C_{n} H_{m}}}+\frac{q W}{M_{C_{p} H_{q}}}\right)\left(Z-Y_{C_{n} H_{m}}-Y_{C_{p} H_{q}}\right)\right. \\
& \left.-2 \frac{Y_{H_{2}}}{M_{H_{2}}}\right\}
\end{aligned}
$$

Similarly, the mass fraction of $\mathrm{O}_{2}$ is calculated from the conservation of oxygen atoms:

$$
Y_{\mathrm{O}_{2}}=\frac{M_{\mathrm{O}_{2}}}{2}\left\{\frac{2 Z(1-W)}{M_{C_{n} \mathrm{H}_{m}}} \frac{\left(n+\frac{m}{4}\right)+\nu\left(p+\frac{q}{4}\right)}{\phi}-2 \frac{Y_{\mathrm{CO}_{2}}}{M_{\mathrm{CO}_{2}}}-\frac{Y_{C O}}{M_{C} \mathrm{O}}-\frac{Y_{\mathrm{H}_{2} \mathrm{O}}}{M_{\mathrm{H}_{2} \mathrm{O}}}\right\}
$$

For equivalence ratios $\phi>1.2$, it is assumed that all the oxygen is consumed during the combustion process:

$$
Y_{O_{2}}=M_{O_{2}} \frac{Z(1-W)}{M_{C_{n} H_{m}}} \frac{\left(n+\frac{m}{4}\right)+\nu\left(p+\frac{q}{4}\right)}{\phi}(1-c)
$$

The mass fraction of water is calculated using Equation (D.9) and the mass fraction of hydrogen using Equation (D.8). In the case that $W=1$, Equations (D.9) and (D.10) must be replaced by Equations (D.11) and (D.12) respectively.

$$
\begin{gathered}
Y_{O_{2}}=\frac{M_{O_{2}}}{2}\left\{\frac{2 Z}{M_{C_{p} H_{q}}} \frac{p+\frac{q}{4}}{\phi}-2 \frac{Y_{C O_{2}}}{M_{C O_{2}}}-\frac{Y_{C O}}{M_{C O}}-\frac{Y_{H_{2} O}}{M_{H_{2} O}}\right\} \\
Y_{O_{2}}=M_{O_{2}} \frac{Z}{M_{C_{p} H_{q}}} \frac{p+\frac{q}{4}}{\phi} c
\end{gathered}
$$

\section{Verification}

The formulation exposed above is verified for one dimensional flames of an hexadecanemethane-air mixture at the same conditions as in Section 4.3.1. The results obtained from Cantera are plotted in Figure D.1 and the equilibrium oxygen and hydrogen mass 


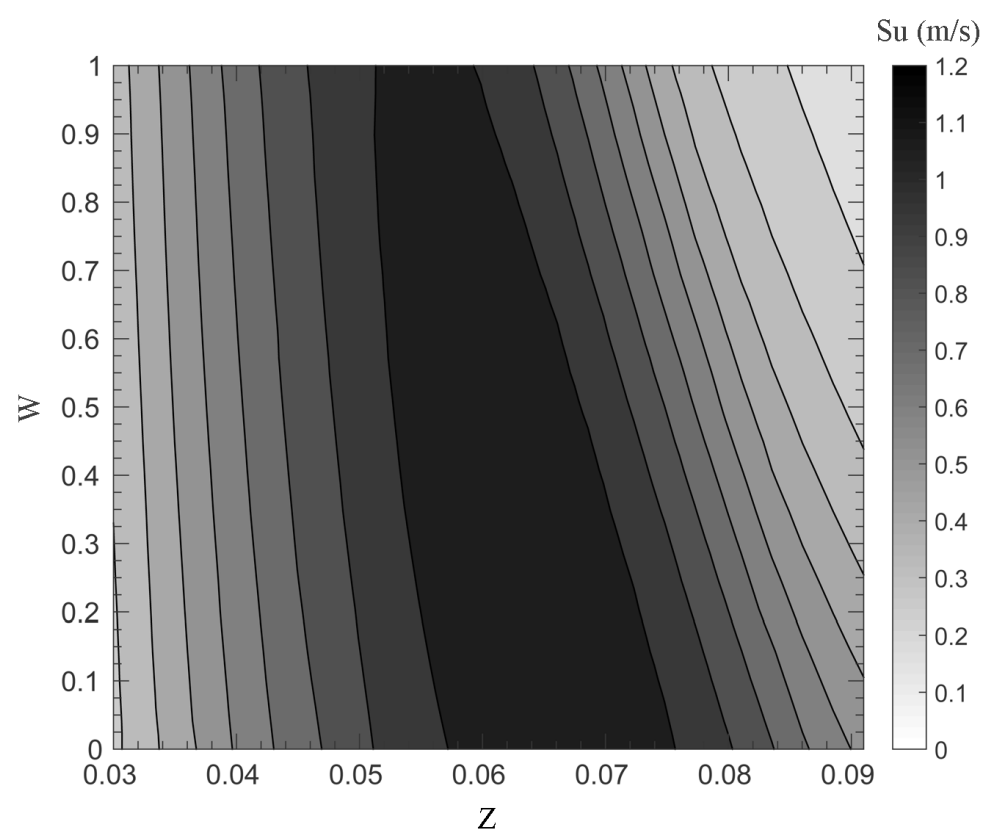

Figure D.1: Effect of methane addition to a hexadecane-air mixture at $P=1$ bar and $T=600 \mathrm{~K}$ on the laminar flame speed.

fractions are shown in Figure D.2 to verify the combustion model assumptions for this kind of mixture.

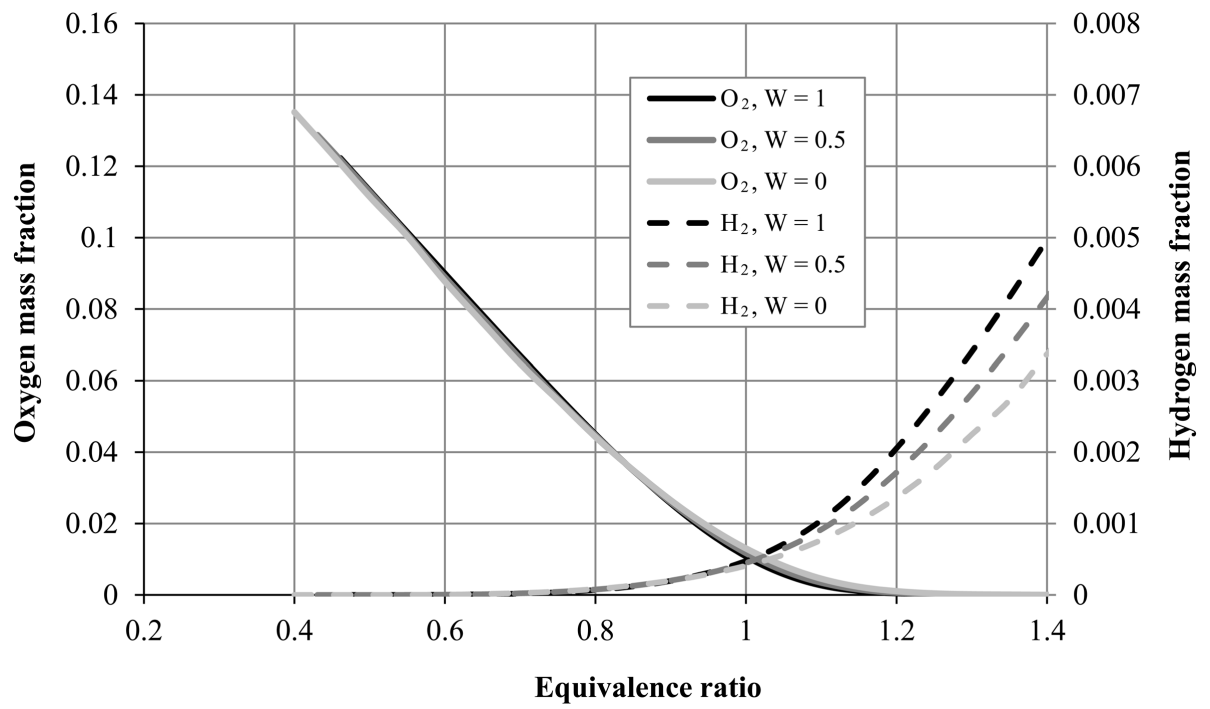

Figure D.2: Post-flame equilibrium oxygen and hydrogen mass fractions for three different methane contents at several equivalence ratios.

In this case, the solution for $W=1$ corresponds to the combustion of a methane-air mixture and therefore the maximum flame speed is located around the stoichiometric fuel mass fraction of methane, which is lower than the one for hexadecane. For a mixture with certain $Z$ and $W$ values, being $W$ lower than unity, if $Z$ is lower than $Z_{S t}$ the increase in the methane content tends to slightly rise the laminar flame speed. On the other hand, if it is higher the flame speed gets significantly reduced. These trends are also visible in 


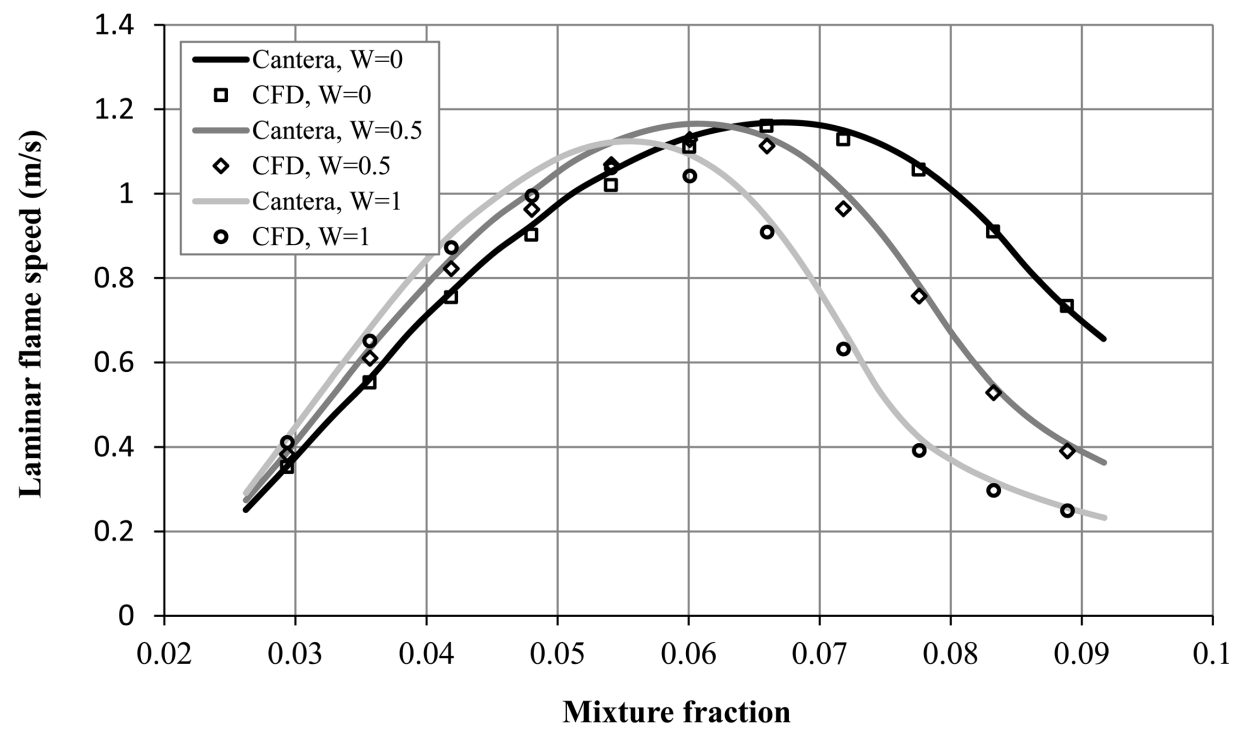

Figure D.3: Comparison of the laminar flame speed between the CFD model and the reference solution from Figure D.1.

Figure D.3, where the flame speeds of the CFD simulations have also been included for comparison. As in the case of water content, the model is able to imitate the shape of the reference curves, particularly for lean and rich mixtures.

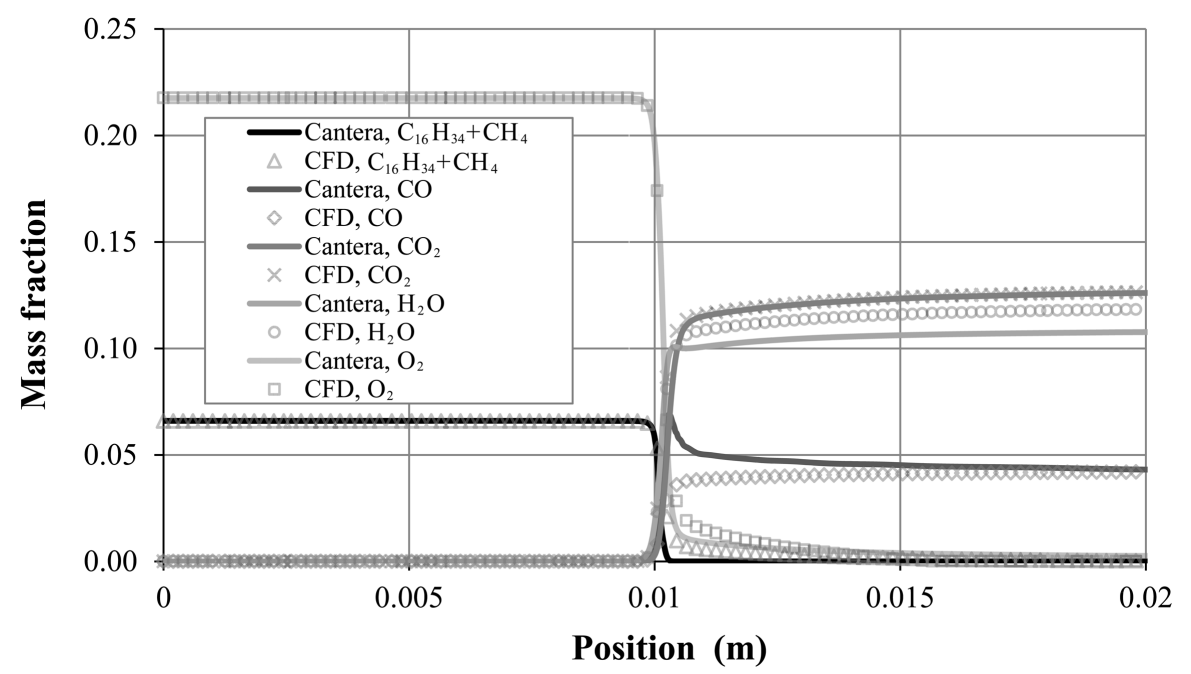

Figure D.4: Comparison of the species profiles between the CFD model and the reference solution from Figure D.1 for $Z=0.063$ and $W=0.5$.

In the region near the stoichiometric fuel fraction, flame speeds are significantly underpredicted. The reason for this is found when observing the profiles of the species' mass fractions, density, velocity and temperature in Figures D.4 and D.5 for the $50 \%$ methane content case. While the $\mathrm{CO}_{2}$ and $\mathrm{CO}$ mass fraction equilibriums are properly predicted, the water mass fraction is over-predicted. The latter causes a slight under-prediction of the oxygen (from Equation (4.16)) and the prediction of a higher temperature than in the reference solution. This reduces the mixture density and, from Equation (4.18), results 


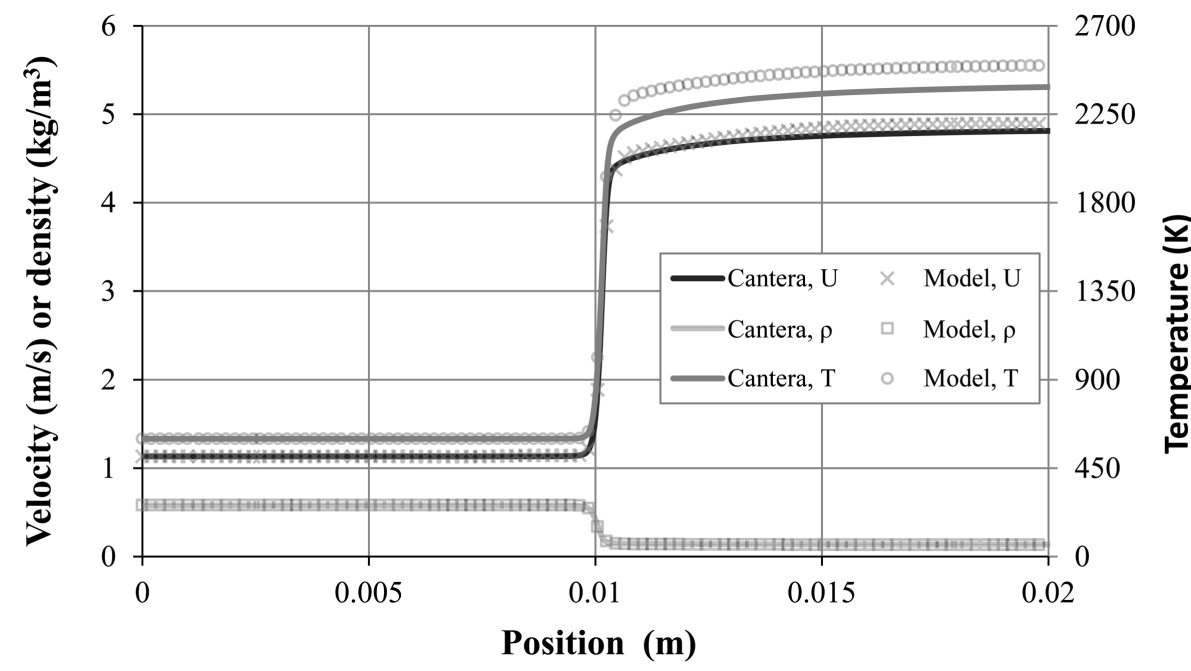

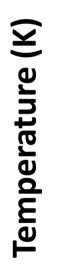

Figure D.5: Comparison of the temperature, density and velocity profiles between the CFD model and the reference solution from Figure D.1 for $Z=0.063$ and $W=0.5$.

in a lower flame speed. The cause for the over-prediction of the water mass fraction lies partly on the fact that hydrogen is already produced for $\phi<1.2$, and that other species like $O H$ are not considered by the model.

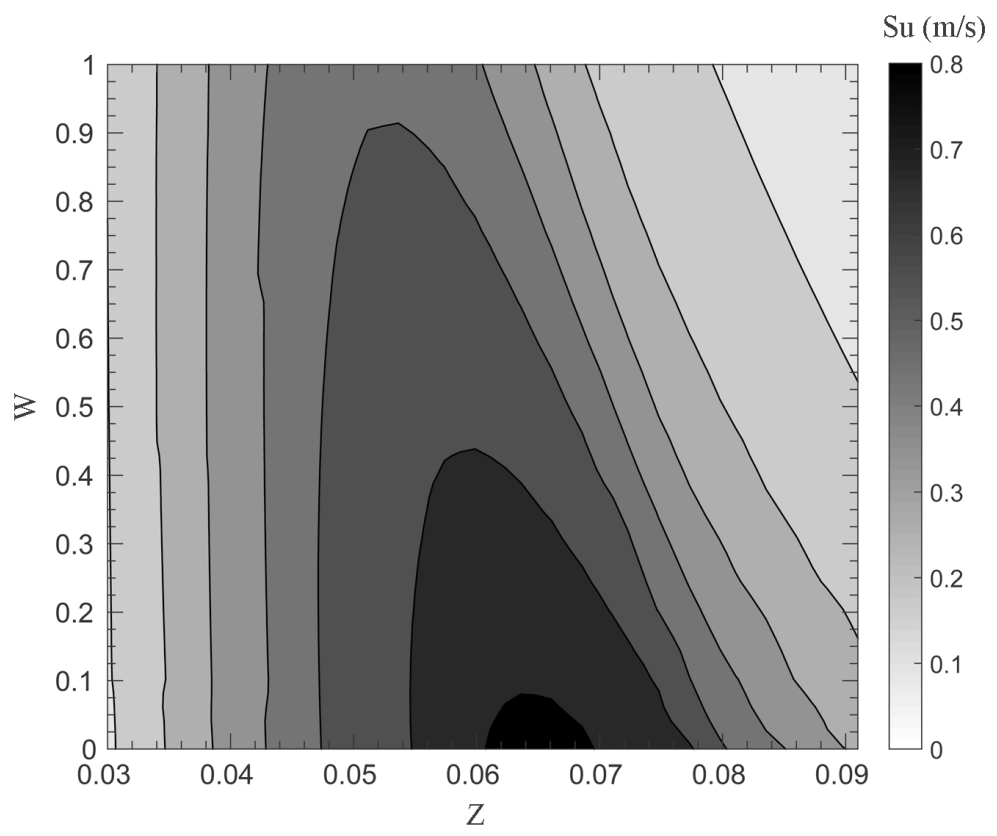

Figure D.6: Effect of methane addition to a hexadecane-air mixture at $P=10$ bar and $T=600 \mathrm{~K}$ on the laminar flame speed.

Graphs for the flame speeds at 10 bar pressure are also plotted in Figure D.6 for an hexadecane-methane-air mixture. It is observed that the increase in pressure has more effect in mixtures with higher methane content. Focusing on Figure D.7, for lean mixtures the flame speed is almost independent of the $W$ variable while for richer mixtures the influence is higher when compared to Figure D.3. In addition, the same trends are fol- 


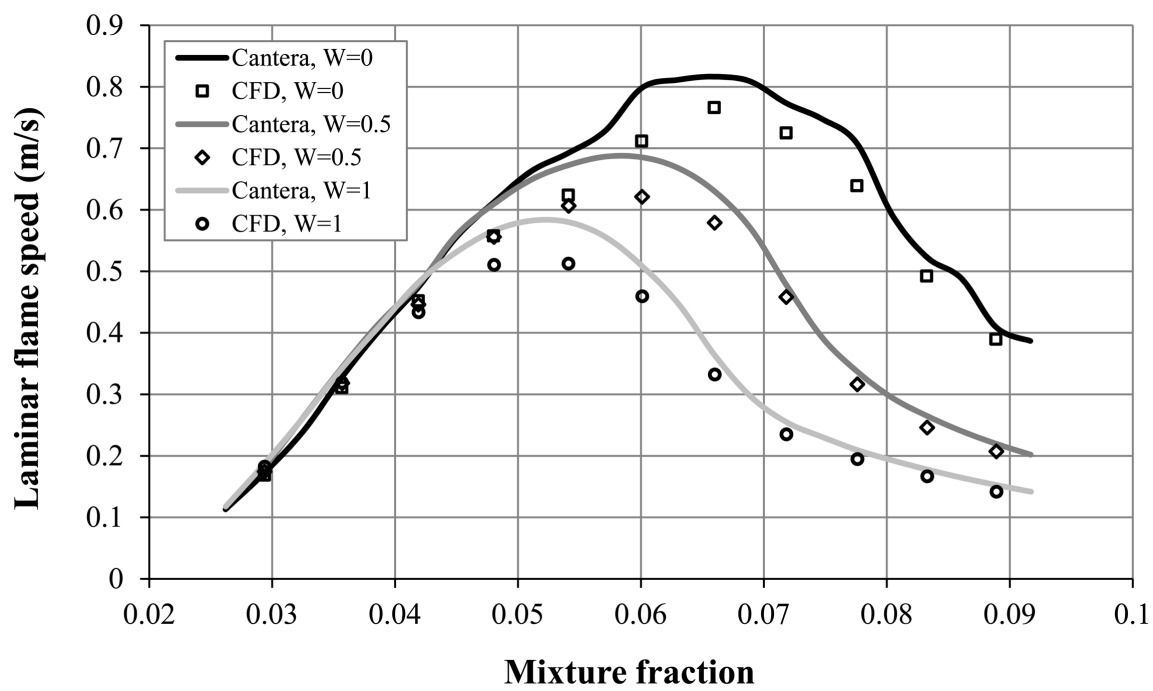

Figure D.7: Comparison of the laminar flame speed between the CFD model and the reference solution from Figure D.6.

lowed by the combustion model, although the flame speed is also under-predicted around stoichiometric conditions as aforementioned.

\section{Mesh sensitivity}

A mesh sensitivity study of a hexadecane laminar flame at $\phi=0.7$ is performed by varying the cell size from 2 to $0.03 \mathrm{~mm}$. The ATF approach implemented by Dederichs [1] is applied. Figure D.8 shows that the flame speed is not greatly affected by the cell size since the maximum deviation is lower than $2 \%$ the one obtained in Cantera. These values are calculated with a time-averaging period of $0.5 \mathrm{~s}$, but amplitude of the flame speed fluctuations increases with the cell size.

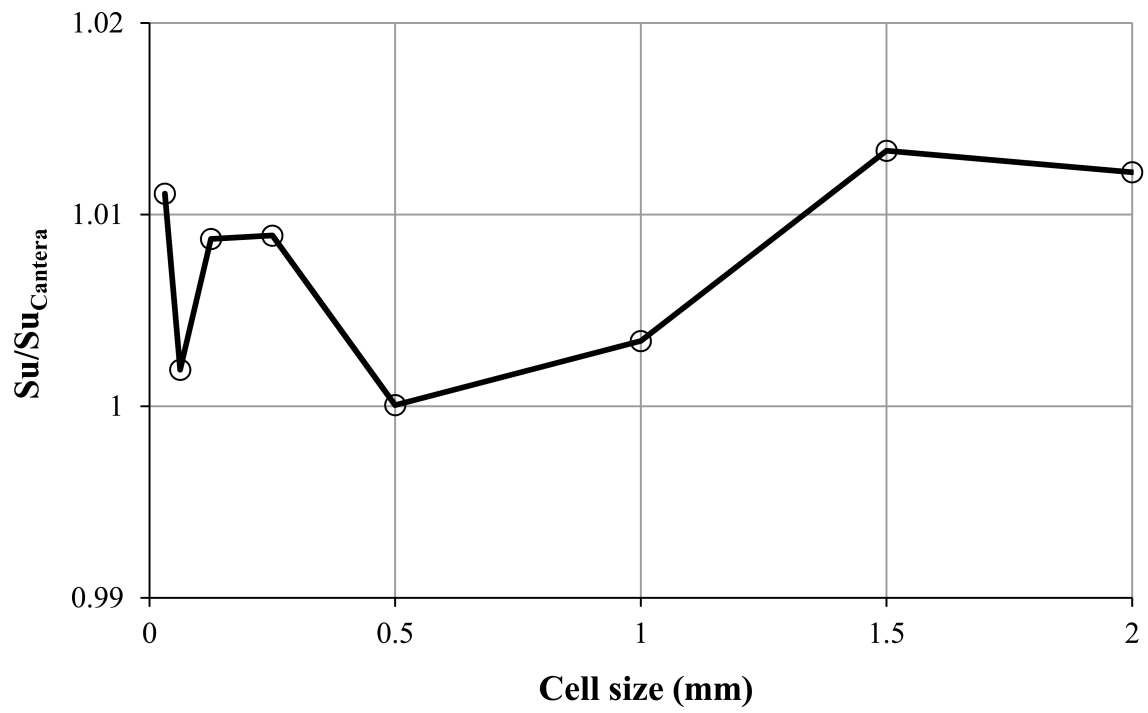

Figure D.8: Mesh sensitivity of the combustion model for a hexadecane 1-D laminar flame at $\phi=0.7, P=1$ bar and $T_{u b}=600 \mathrm{~K}$. 


\section{Appendix E}

\section{Simulation of the Siemens scaled can burner}

A collaborative work was carried out with the Barcelona Supercomputing Center, a partner of the COPA-GT project. It is focused on the modelling of the reacting flow field in the downscaled Siemens can combustor, which has been previously investigated experimentally at the DLR [1], using LES.

Although experimental data is available at different operating conditions, the numerical simulations will be focused on the full load condition at 4 bar with preheated air and 1.2 MW thermal power burning pure methane. The combustor consists of a main burner with 8 swirling injectors and a central conical-shaped pilot burner. Further details of the geometry can be found in the work by Lückerath et al. [1]. This combustor has already been investigated numerically by Goldin et al. [2] in adiabatic conditions using RANS and LES, providing acceptable correlation with the experimental data when using the FGM combustion model. The present work aims to simulate the same operating point, but investigates the effect of heat losses on the prediction capabilities of the codes.

The heat losses are accounted for by imposing isothermal heat transfer conditions at the wall chamber and the cone walls, where the flame is anchored to the pilot. Due to the strong interaction between the pilot and the main regions, this thermal condition is expected to affect the position where the flame stabilizes at both the wall chamber and the cone.

The numerical simulations are conducted with Alya [3] and OpenFOAM [4], which are two different well-established CFD codes in the fluid mechanics community. Alya is a parallel multi-physics CFD code of the PRACE Benchmark Suite for HPC applications. 


\section{Alya modelling approach}

The code Alya uses a spatial discretization based on the Finite Element method using the Variational Multiscale Stabilization technique [5] and the subgrid scale effects are accounted for by the WALE model [6]. A second-order Crank-Nicholson time integration scheme is used for the LES simulations.

The non-premixed database is generated from laminar premixed flamelets using the code PREMIX [7]. The creation of the manifolds $\psi(Z, c)$ differs depending on the flammability region. While outside flammability limits, mixture averaged properties are used, laminar premixed flamelets obtained with PREMIX are generated for each value of mixture fraction. The definition of the progress variable is based on the CSP method, which obtains a $b$-vector that weights the contribution of each species on the reaction progress variable [8]. The definition of the progress variable is given by:

$$
\begin{gathered}
Y_{r p v}=\sum_{k=1}^{N} b_{k} Y_{k} \\
c=\frac{Y_{r p v}-Y_{r p v, u b}}{Y_{r p v, b}-Y_{r p v, u b}}
\end{gathered}
$$

where $\mathrm{N}$ is the total number of species used by the GRI 3.0 mechanism [9]. The use of the CSP approach results into a more uniform distribution of the source term along the span of the reaction progress variable, allowing larger time-steps and reducing the stiffness of the chemistry calculation [8].

\section{Results and discussion}

The results of the simulations are presented in this section. After the test case is introduced, details of the mesh for the full geometry are provided along with the comparison of the numerical results against the experimental data. The key physical phenomena are also discussed.

\section{Experimental test case}

The burner consists of eight main swirl burners arranged on an annulus with a pilot swirl burner at the center. The fuel staging uses two fuel lines, which directly inject into the swirling vanes of the main and pilot burners. The combustor also includes an ignition zone that was also considered in the simulations. The length of the combustion chamber is $380 \mathrm{~mm}$, while the rectangular cross section is $95 \times 95 \mathrm{~mm}^{2}$. Double-glassed windows with air cooling are used for the combustion chamber walls. The inlet air is preheated up to $704 \mathrm{~K}$, while cold fuel is injected at a temperature of about $363 \mathrm{~K}$. The numerical simulations consider the conditions of the combustor operated at $p=4$ bar 
Table E.1: Conditions imposed at the inlet and outlet patches.

\begin{tabular}{c||ccccc} 
& Main $_{\text {air,in }}$ & Main $_{\text {fuel,in }}$ & Pilot $_{\text {air,in }}$ & Pilot $_{\text {fuel,in }}$ & Outlet \\
\hline \hline $\mathrm{T}(\mathrm{K})$ & 704 & 363 & 704 & 553 & - \\
$\dot{m}(\mathrm{~g} / \mathrm{s})$ & 636.3 & 19.9 & 70.7 & 2.71 & - \\
$Z$ & 0 & 1 & 0 & 1 & - \\
$\mathrm{P}_{\text {out }}$ (bar) & - & - & - & - & 4
\end{tabular}

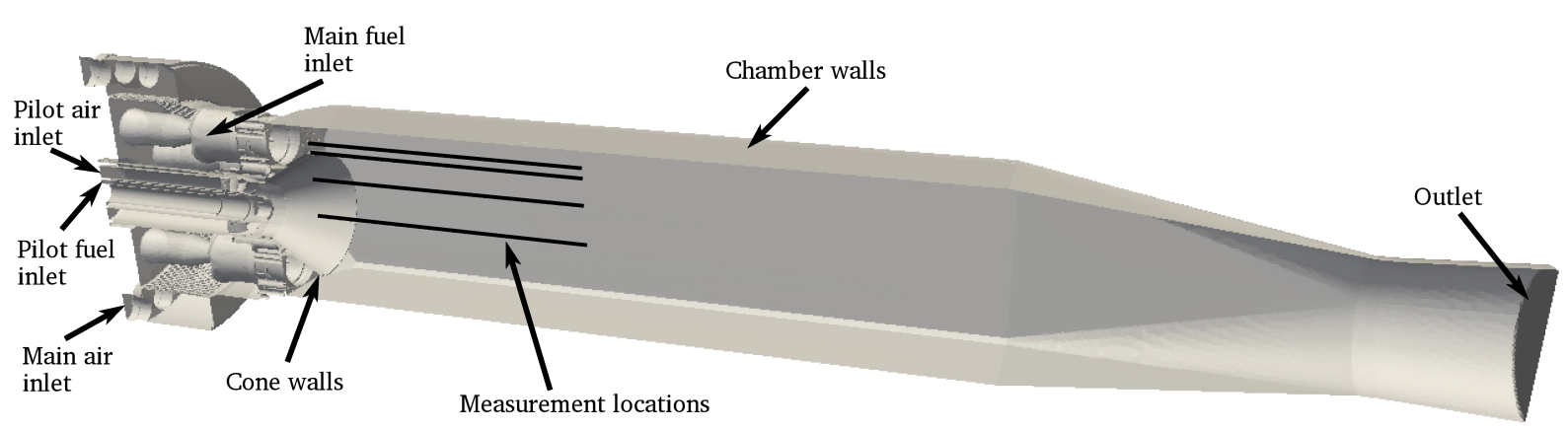

Figure E.1: Geometry and computational boundaries.

with a maximum thermal load of 1.2 MW. The field values imposed at each patch are summarized in Table E.1 and an sketch of the combustor is depicted in Figure E.1. The measurement locations where the comparison with the experimental data takes place are also shown. The experimental data available for comparison is given at three radial positions and along the streamwise direction of the combustor. The values for mixture fraction and temperature correspond to the $y=0,20,34 \mathrm{~mm}$ lines, while the experimental data for the axial velocity is given at the $y=0,20,39 \mathrm{~mm}$ locations.

\section{Simulation setup}

The computational mesh employed by Alya to solve the problem is composed by 47 million tetrahedrons and has been refined along the multi-perforated plates as well as at the central pilot and the surrounding burners where the swirlers are located. The mesh used in OpenFOAM has about 32 million cells: 30 million hexahedrons, 1.5 million polyhedrons, 0.5 million prisms and few tetrahedrons. It was not possible to use this mesh with Alya due to the incompatibility of the polyhedrons with the finite element method, while numerical issues occurred when running OpenFOAM with the full tetrahedron mesh. Details of these meshes can be seen in Figure E.2. A zoom of the mesh near the pilot fuel injection and the main swirlers is included on the right-hand side plot of Figure E.2 for both grids.

The numerical investigation assumes adiabatic conditions for all the walls except the cone and combustion chamber walls, where uniform temperatures of $1500 \mathrm{~K}$ and $1000 \mathrm{~K}$ 

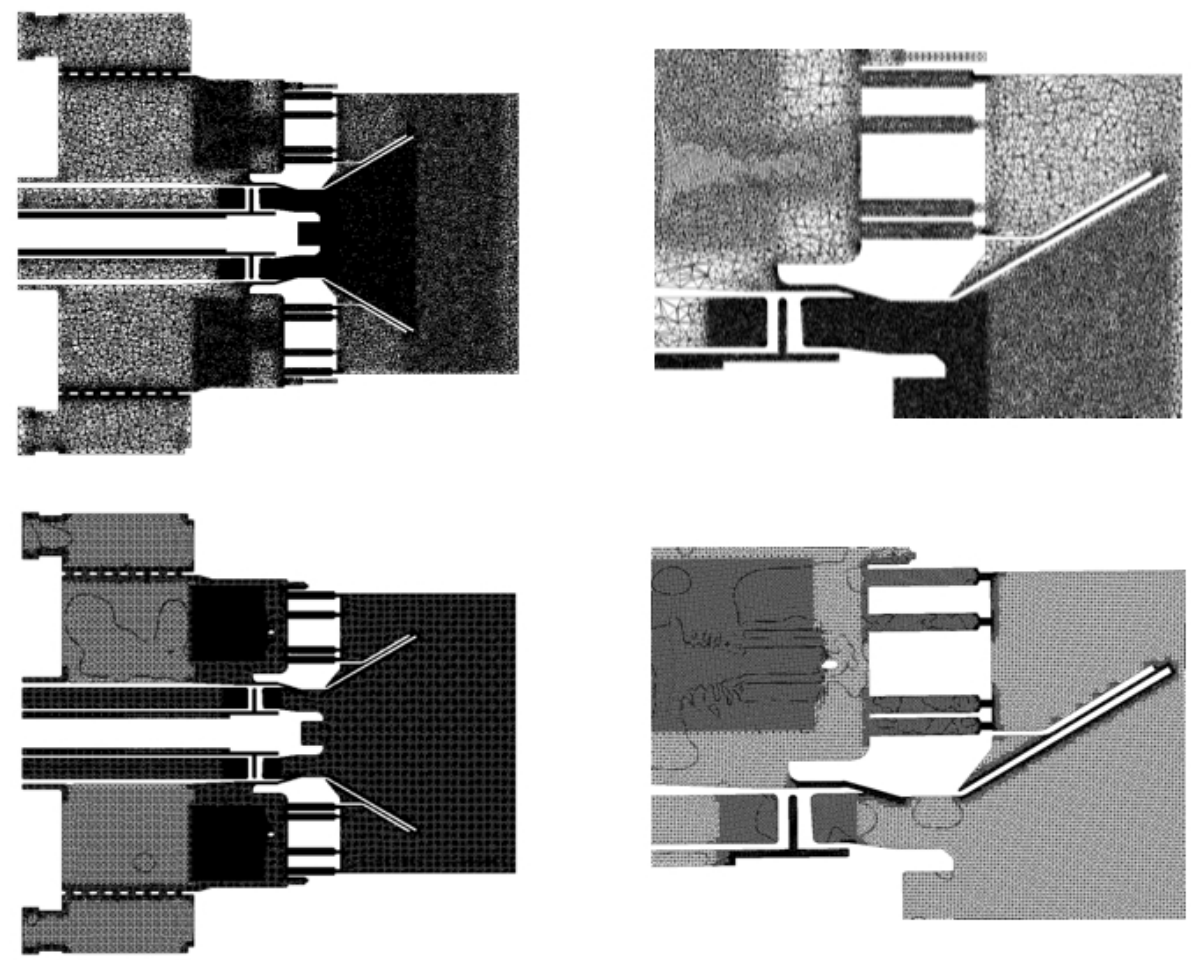

Figure E.2: Z-plane of the computational meshes: Alya (top) and OpenFOAM (bottom). are imposed respectively. The objective of this thermal condition is to model the heat losses from the flame to the walls, which affect the flame position and stability.

\section{Numerical results}

Numerical LES simulations have been conducted with the two codes for the configuration shown in Figure E.1. The combustor operates in the lean regime with a equivalence ratio around $\phi=0.55$. The flame is stabilized in the burner along the prolongation of the central cone walls. The flame is anchored in this region because of the wake of the pilot cone, but also due to the addition of the air that is employed for cooling pilot cone. This results in a zone with high enthalpy, which promotes a higher flame speed when the hot air is mixed with the fuel. The LES results are time-averaged for three residence times and mean values for velocity, temperature and mixture fraction are compared with the experimental data from Lückerath et al. [1].

Time-averaged results of the axial velocity for the two codes are shown in Figure E.3 for comparison. The first discrepancy between the codes is at the main air inflow, since Alya predicts a higher velocity of the flow before passing the perforated plates. Although the flow through the swirlers is not compared, since it is not visible in the plane selected, the axial velocities at the end of the pilot cone are in good agreement. The same can be stated for the air in the pilot passage. The length and width of the recirculation zone predicted by the two codes are comparable, although Alya predicts a stronger 

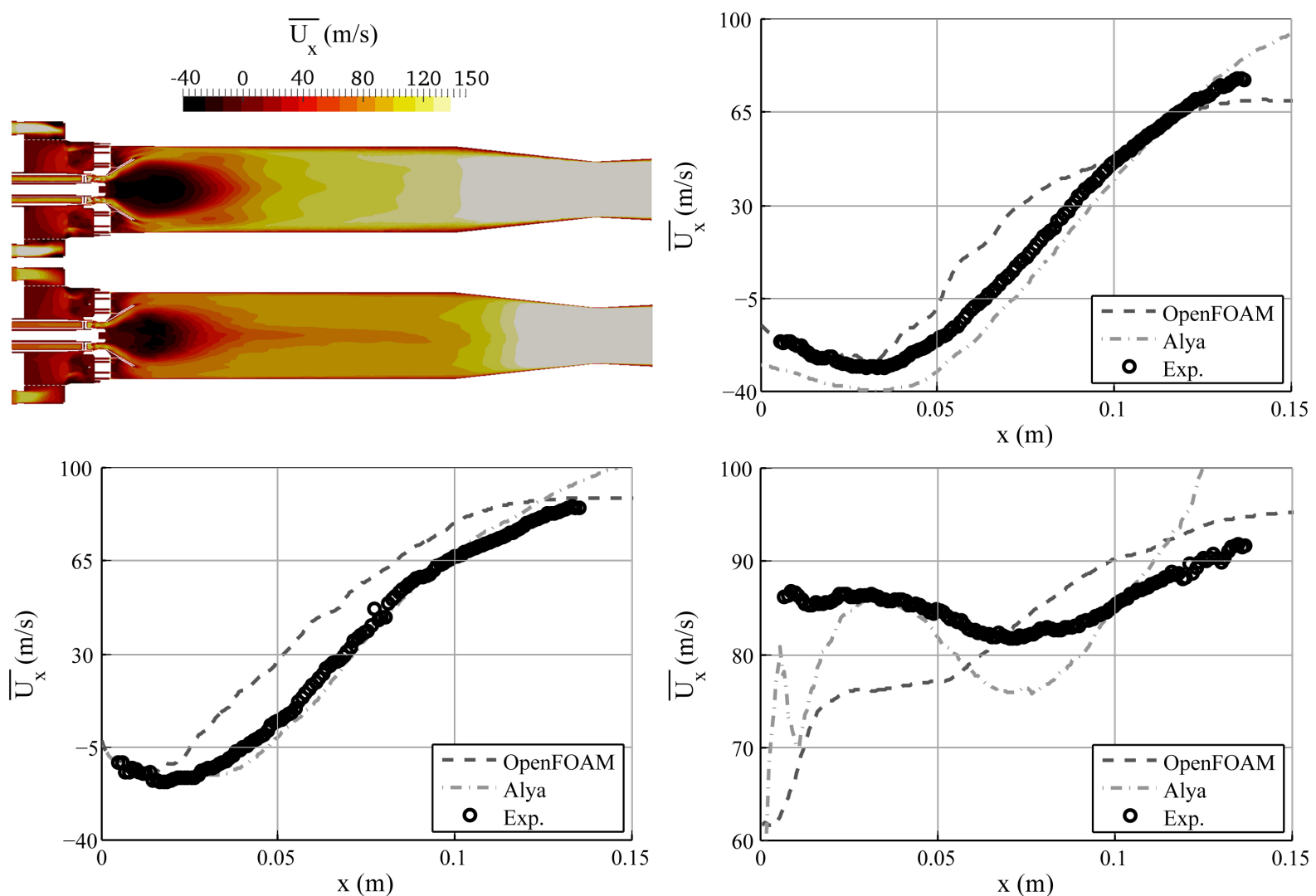

Figure E.3: Comparison of the axial velocity contours (top left, Alya over OpenFOAM) and profiles with experimental data at $y=0 \mathrm{~mm}$ (top right), $y=20 \mathrm{~mm}$ (bottom left) and $y=34 \mathrm{~mm}$ (bottom right).

recirculation than OpenFOAM. The axial velocity predicted by Alya at the rear of the chamber is significantly higher than the one computed by OpenFOAM. The reason for this discrepancy can be seen in Figure E.4, where the time-averaged mixture fraction is shown. The mixture fraction field is an important quantity that defines the distribution of fuel/air mixture in the combustor and, together with the enthalpy provides the thermal state of the system. In general, a uniform mixture fraction is predicted inside the chamber except at the pilot cone region, where it is sensibly higher in order to stabilize the combustor. When comparing the solutions, Alya predicts a slightly higher mixture fraction than OpenFOAM and a thicker boundary layer at the combustor walls. This produces a higher temperature, due to the combustion of a richer mixture, and a smaller effective area which results in the velocity differences shown in Figure E.3. The axial velocity profiles on the measurement lines for both codes and in the experiments are also included in the figure. The comparison indicates that a good correlation level is obtained in the two inner lines of the combustor for both codes, especially with Alya. OpenFOAM predicts a slightly higher axial velocity, with a maximum deviation of $20 \mathrm{~m} / \mathrm{s}$.

Comparison of the mixture fraction results are shown in Figure E.4. Both codes predics a fully premixed mixture within the combustion chamber and the main differences are 

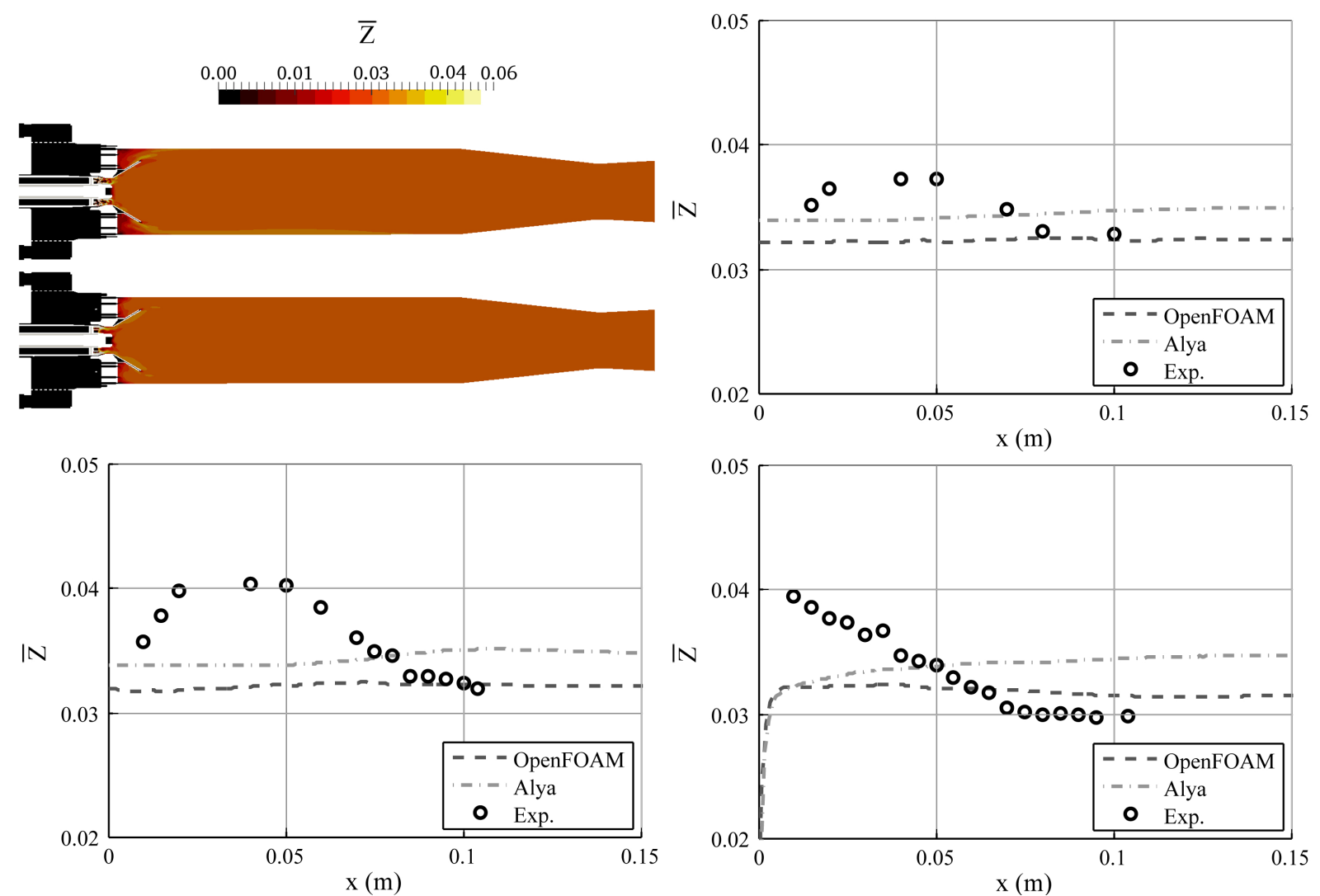

Figure E.4: Comparison of the mixture fraction contours (top left, Alya over OpenFOAM) and profiles with experimental data at $y=0 \mathrm{~mm}$ (top right), $y=20 \mathrm{~mm}$ (bottom left) and $y=34 \mathrm{~mm}$ (bottom right).

located upstream of the cone, where OpenFOAM predicts a higher mixture fraction value, and in the mixing region downstream of the pilot swirler. The experimental data at the three measurement locations shows that fuel and air are not fully premixed, but there is a rich region within the recirculation zone. Despite this is not captured in the simulations, a good level of correlation is obtained. As aforementioned, Alya predicts a slightly higher mixture fraction than OpenFOAM. This leads to a better agreement with the experiments for Alya within the richer region (i.e. $x<0.08 \mathrm{~m}$ ) and for OpenFOAM downstream of it.

Similarly, the temperature distribution is plotted in Figure E.5. The most significant difference between the codes is that, in the burned side, Alya predicts a higher temperature level than OpenFOAM because of the greater mixture fraction. The shape of the flames is also different. The pilot flame is shorter with OpenFOAM while shape of the main flame is slightly longer and moves towards the center with Alya. Additionally, the low temperature region near the chamber wall - due to the isothermal conditions imposed - is thicker in the Alya simulation, which explains the greater boundary layer thickness observed in the axial velocity contours.

The comparison with the experiments indicates that a good agreement is obtained in the first two lines, with a better prediction for OpenFOAM at $y=0$ and for Alya at 

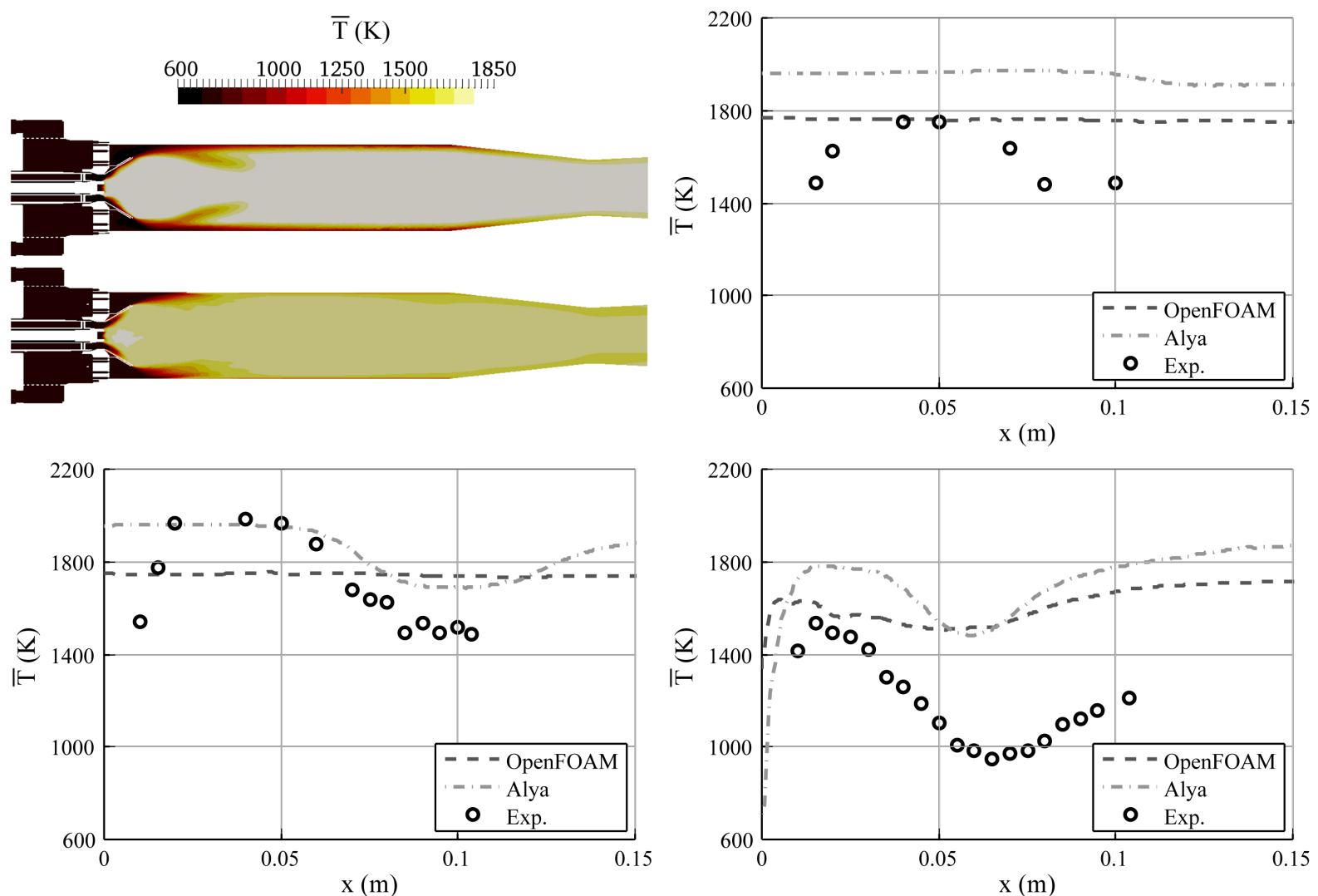

Figure E.5: Comparison of the temperature contours (top left, Alya over OpenFOAM) and profiles with experimental data at $y=0 \mathrm{~mm}$ (top right), $y=20 \mathrm{~mm}$ (bottom left) and $y=39 \mathrm{~mm}$ (bottom right).

$y=20 \mathrm{~mm}$. Particularly, Alya is able to reproduce the decrease in temperature between $x=50 \mathrm{~mm}$ and $x=100 \mathrm{~mm}$. This supports the shape of the main flame obtained with Alya. At $y=34 \mathrm{~mm}$, temperatures are significantly over-predicted by both codes.

In general, both codes provide a solution that are different in some details but they show similar trends. Except for few lines, a good level of agreement is obtained for the axial velocity, mixture fraction and temperature.

\section{References}

[1] R. Lückerath et al. "Experimental investigations of flame stabilization of a gas turbine combustor". In: ASME Turbo Expo 2011. GT2011. 45790. 2011.

[2] G. Goldin, F. Montanari and S. Patil. "A Comparison of RANS and LES of an Industrial Lean Premixed Burner". In: ASME Turbo Expo 2014. GT2014. 25352. 2014.

[3] BSC-CNS. Alya multiphysics code. 2014. URL: http://www.bsc.es/alya. 
[4] H. G. Weller et al. "A tensorial approach to computational continuum machanics using object-oriented techniques". In: Computers in Physics 12.6 (1998), pp. 620631.

[5] G. Houzeaux and J. Principe. "A variational subgrid scale model for transient incompressible flows". In: Int. J. Comput. Fluid D. 22 (2008), pp. 135-152.

[6] F. Nicoud and F. Ducros. "Subgrid-scale stress modelling based on the square of the velocity gradient". In: Flow, Turbulence and Combustion 62 (1999), pp. 183-200.

[7] R.J. Kee et al. A Fortran Computer Code Package for the Evaluation of Gas-Phase Multicomponent Transport Properties. Tech. rep. SAND86-8246. Sandia National Laboratories, 1986.

[8] S. Gövert et al. "Turbulent combustion modeling of a confined premixed methane/air jet flame including heat loss effects using tabulated chemistry". In: Appl. Energ. 156 (2015), pp. 804-815.

[9] G. P. Smith et al. GRI-Mech 3.0. The Gas Research Institute. URL: http: //www . me.berkeley.edu/gri_mech/. 


\section{Appendix F}

\section{Jet flame simulation with the coarse grid and enthalpy clipping}

As a reference for the acoustic analysis and to evaluate the mesh sensitivity of this configuration, the non-excited Baseline and Diesel jet flames from Chapter 6 are simulated with the coarse mesh shown in Figure 7.1. Results are compared to those obtained with the fine mesh, displayed in Figure 6.4, some of which were in turn compared to experimental data in Sections 6.4.1 and 6.4.2.

\section{Baseline}

The velocity distribution within the premixing duct is plotted in Figure F.1. The axial contours show that boundary layer is thicker for the coarse mesh. Furthermore, there is a preferential direction at $45^{\circ}$ from the Cartesian axis while a more symmetric distribution is obtained with the fine mesh. As a result, the coarse grid leads to a significantly higher bulk jet velocity.

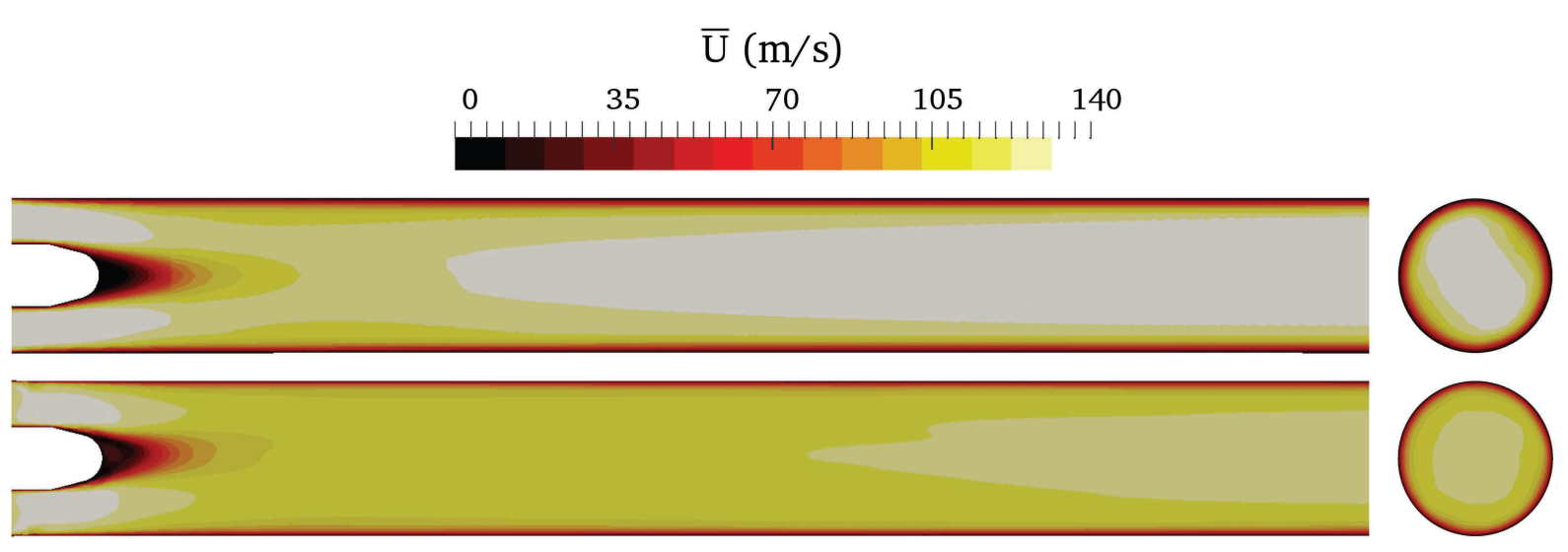

Figure F.1: Time-averaged velocity fields at a mid-plane of the premixing duct computed with the coarse (top) and fine (bottom) meshes. The axial contours are located $50 \mathrm{~mm}$ upstream of the chamber baseplate. 
The quantitative comparison of the axial velocity within the combustion chamber at the same lines as in Figure 6.9 is presented in Figure F.2.
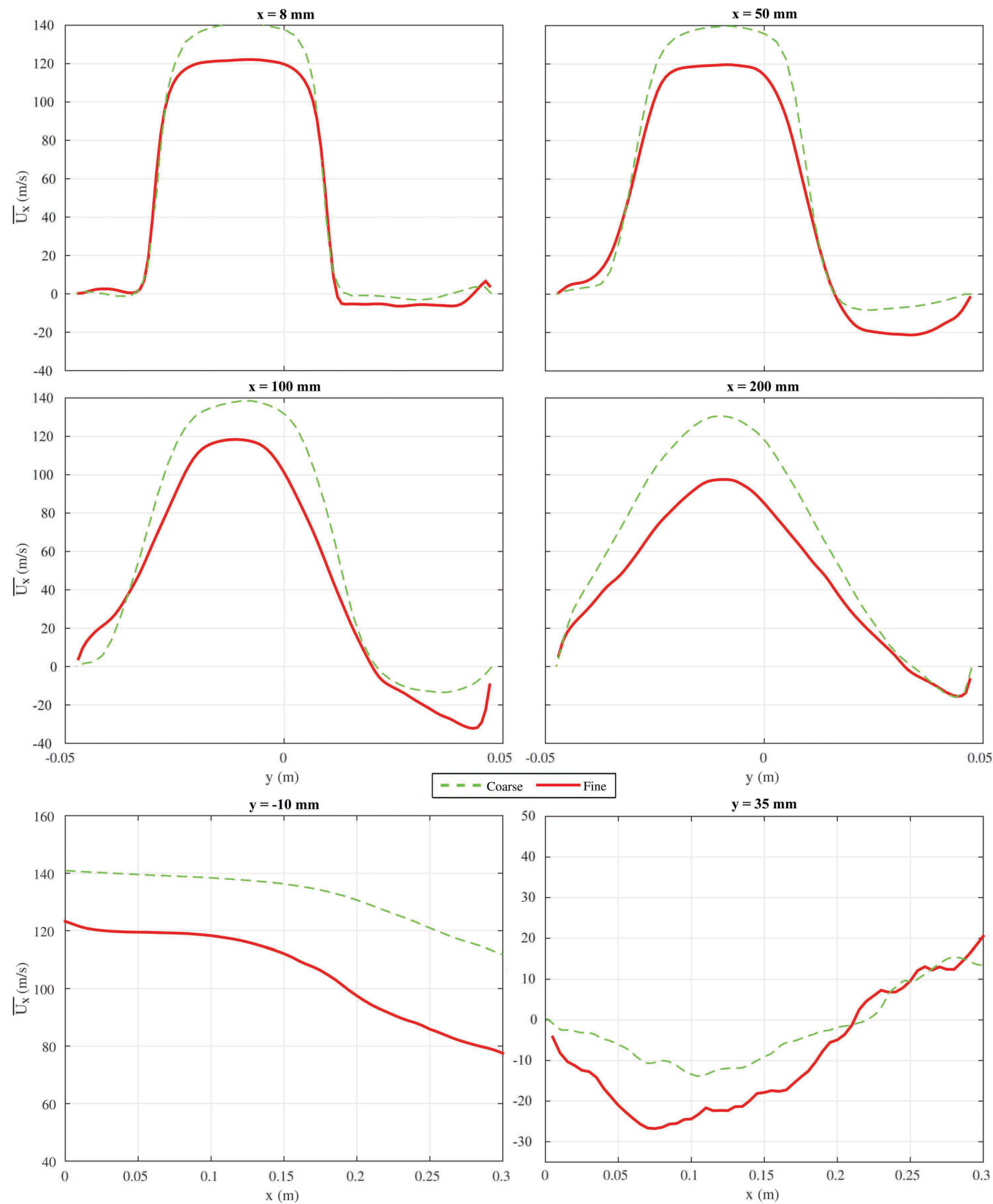

Figure F.2: Comparison of the axial velocity profiles simulated with the coarse and fine meshes at four axial lines, downstream of the baseplate, and two longitudinal lines at the jet center and at the middle of the large recirculation region.

The transversal lines show that the jet velocity is between 20 and $30 \mathrm{~m} / \mathrm{s}$ larger for the coarse mesh. The spread of the profiles with the axial distance is lower than for the fine 
grid, since the slope difference at the shear layer region increases from the first to the last line. This implies that the difference in the peak velocity also rises with the distance from the baseplate, which is also observed in the $y=10 \mathrm{~mm}$ line. The recirculation strength is smaller for the coarse mesh, being the maximum discrepancy around $15 \mathrm{~m} / \mathrm{s}$.

Since the mixture that enters the combustion chamber is technically premixed, contours for fuel distribution are not generated. The flame shape and intensity for both grids are plotted in Figure F.3. Since the $\mathrm{CO}_{2}$ source term depends on the cell size, the width-integrated heat release distribution - normalized with the injected fuel mass flow times the LHV - is used in this comparison. The flame with coarse grid is slightly longer, due to the larger jet velocity, and more distributed. The latter is partly caused by the clipping of the enthalpy to the lowest from the tables, which results in a flame that is attached to the baseplate, but most of the heat is release at the flame tip. In contrast, the flame lifts with the assumption of zero progress variable source term and it leads to more concentrated and intense flame. This affect the temperature distributions, which are plotted in Figure F.4, so that a higher temperature downstream of the flame is obtained for the fine grid.

\section{Norm. Heat Release}

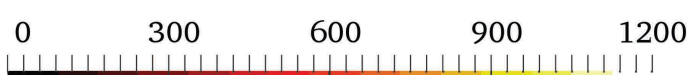
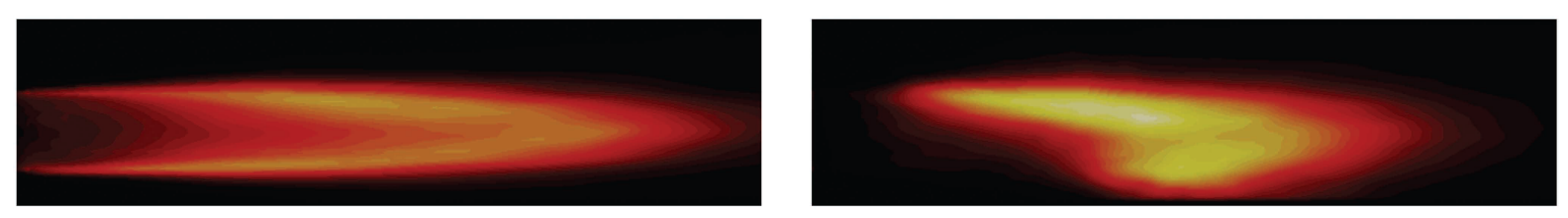

Figure F.3: Width-integrated normalized heat release of the Baseline case with the coarse (left) and fine (right) grids. If the local enthalpy is lower than the lowest value from the tables, it clipped for the coarse simulation while the local progress variable source term is set to zero for the fine simulation.

\section{Diesel}

The global spray characteristics at six planes within the premixing duct are compared in Figure F.5. The liquid mass flow is slightly lower for the coarse mesh due to a larger amount of mass vaporized from the first to the second plane, since the difference reduces at the following planes. This is caused by a faster heat-up, due to the larger convective heat transfer because of the higher gas phase velocity, since the maximum difference in the SMD is approximately $5 \mu \mathrm{m}$. The spray velocity at the front planes is smaller than the one with the fine grid, which indicates that the droplet-wall interaction starts at a more upstream position. 


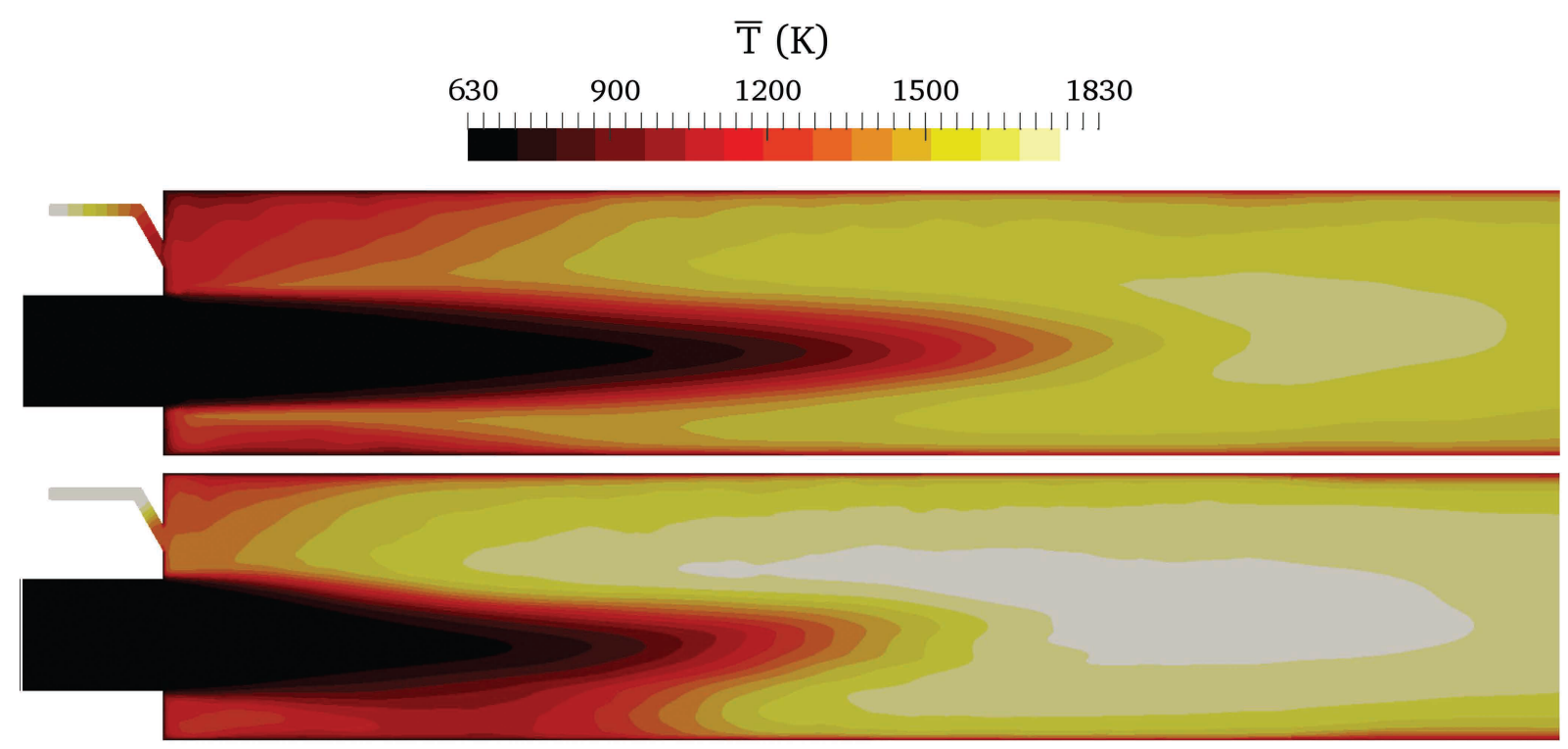

Figure F.4: Time-averaged temperature fields at a mid-plane of the combustion chamber obtained with the coarse (top) and fine (bottom) meshes.
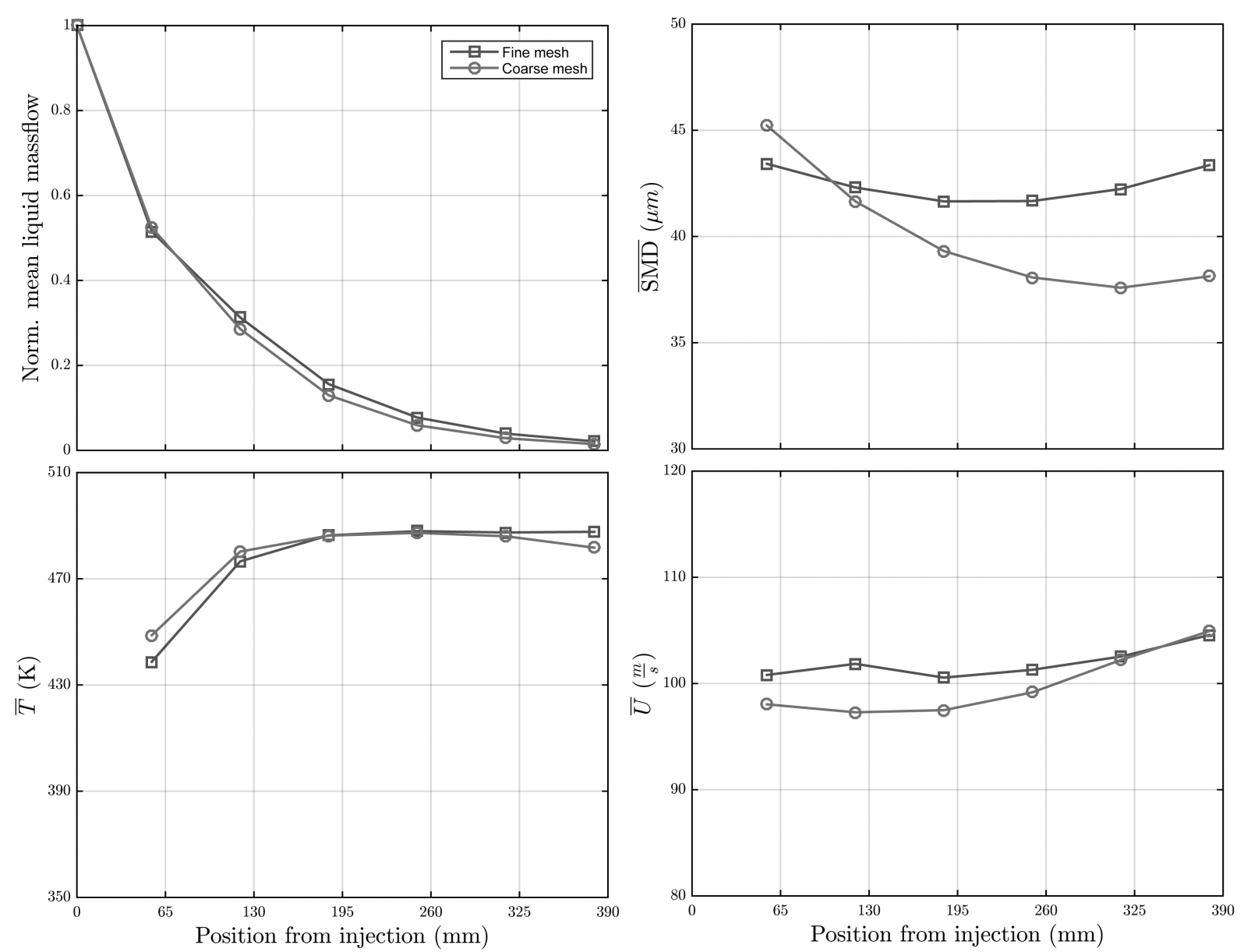

Figure F.5: Evolution of the global time-averaged spray mass flow (top left), SMD (top right), temperature (bottom left) and velocity (bottom right) along the mixing duct for the dry oil case employing the coarse and the fine meshes. 
The mixture fraction distributions within the premixing duct are depicted in Figure F.6. It shows that there is a significantly higher amount of vaporized fuel at the duct walls, especially in the preferential direction, which arises at a shorter distance from the injection than with the fine grid. Thus, a the fuel mass fraction at the centre of the duct is smaller. Similarly, the distributions within the combustion chamber are plotted in Figure F.7. A more inhomogeneous fields can be observed for the coarse mesh. The mixture that enters from the duct walls, which spreads to the sides of the jet, is very close to stoichiometric conditions $\left(Z_{s t} \approx 0.065\right)$.

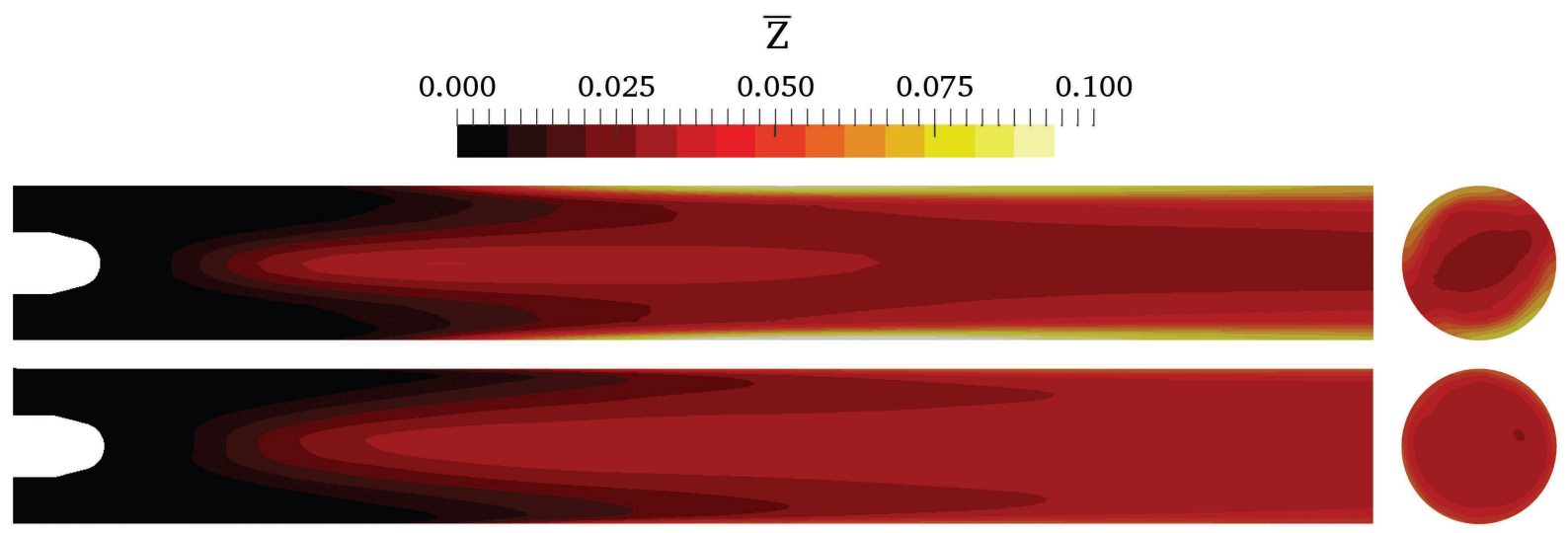

Figure F.6: Time-averaged mixture fraction distributions at a mid-plane of the premixing duct obtained with the coarse (top) and fine (bottom) meshes.

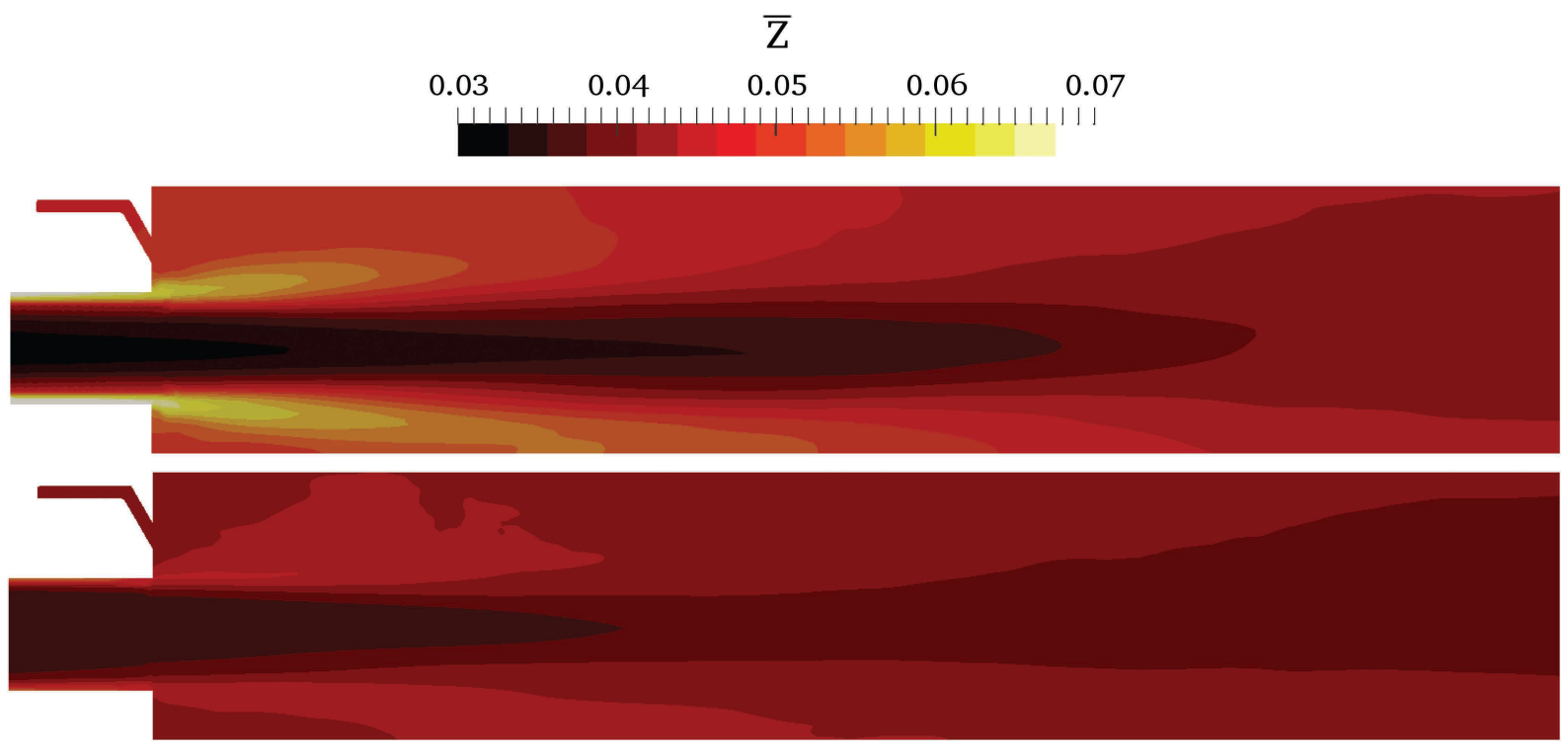

Figure F.7: Time-averaged mixture fraction distributions at a mid-plane of the combustion chamber obtained with the coarse (top) and fine (bottom) meshes.

The fuel distribution presented above results in a flame where a larger amount of the heat is released at the flame root, as shown in Figure F.8. Despite the larger flow velocity, the flame is shorter since there is less fuel concentrated at the jet centre. The enthalpy clipping also contributes to a more stable and shorter flame. Additionally, the temperature 
profiles are plotted in Figure F.9. It shows that there is a significant discrepancy between fine and coarse meshes in terms of the flame temperature at the sides of the jet (greater than $200 \mathrm{~K})$.

\section{Norm. Heat Release}
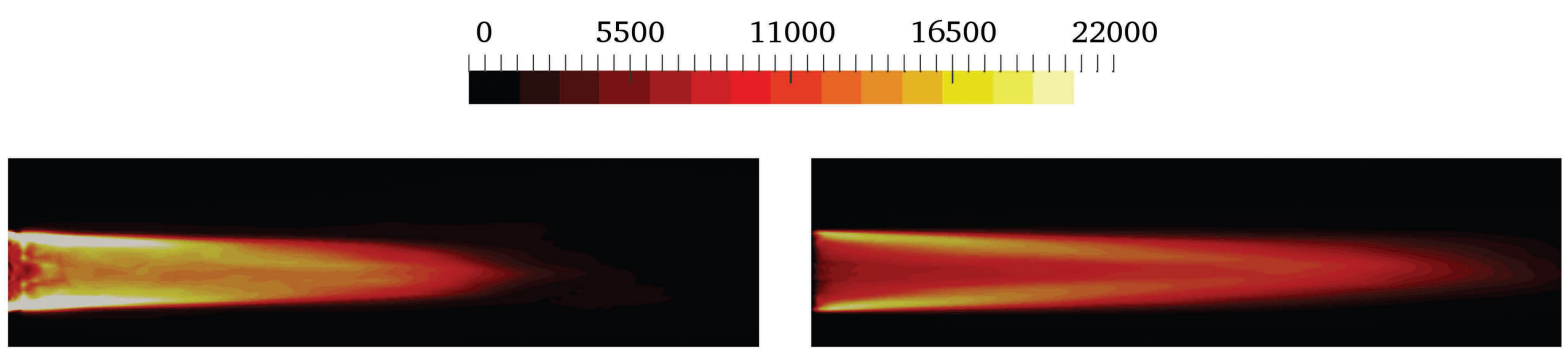

Figure F.8: Width-integrated normalized heat release of the Diesel case with the coarse (left) and fine (right) grids. If the local enthalpy is lower than the lowest value from the tables, it clipped for the coarse simulation while the local progress variable source term is set to zero for the fine simulation.

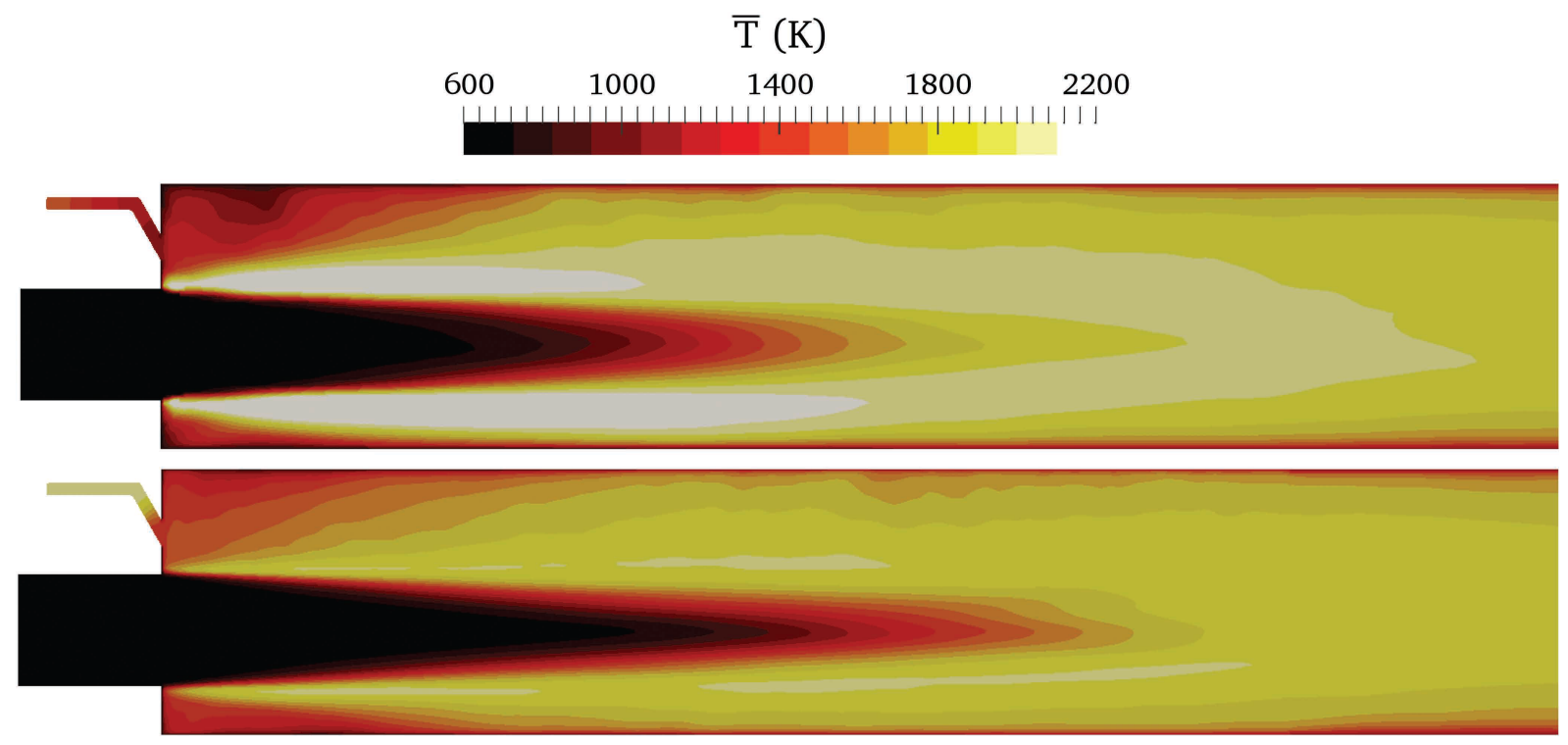

Figure F.9: Time-averaged temperature fields at a mid-plane of the combustion chamber obtained with the coarse (top) and fine (bottom) meshes.

Thus, compared to the gas results, liquid fuel simulations are more grid dependent because the fuel distribution can vary significantly due to the changes in the velocity field that affect the spray spatial location. 